\title{
Distributions in CFT. Part II. Minkowski space
}

\author{
Petr Kravchuk, ${ }^{a}$ Jiaxin Qiao ${ }^{b, c}$ and Slava Rychkov ${ }^{c, b}$ \\ ${ }^{a}$ Institute for Advanced Study, \\ Princeton, New Jersey 08540, U.S.A. \\ ${ }^{b}$ Laboratoire de Physique de l'Ecole normale supérieure, ENS, \\ Université PSL, CNRS, Sorbonne Université, Université de Paris, \\ F-75005 Paris, France \\ ${ }^{c}$ Institut des Hautes Études Scientifiques, \\ 91440 Bures-sur-Yvette, France \\ E-mail: pkravchuk@ias.edu, jiaxin.qiao@phys.ens.fr
}

ABSTRACT: CFTs in Euclidean signature satisfy well-accepted rules, such as the convergent Euclidean OPE. It is nowadays common to assume that CFT correlators exist and have various properties also in Lorentzian signature. Some of these properties may represent extra assumptions, and it is an open question if they hold for familiar statistical-physics CFTs such as the critical 3d Ising model. Here we consider Wightman 4-point functions of scalar primaries in Lorentzian signature. We derive a minimal set of their properties solely from the Euclidean unitary CFT axioms, without using extra assumptions. We establish all Wightman axioms (temperedness, spectral property, local commutativity, clustering), Lorentzian conformal invariance, and distributional convergence of the s-channel Lorentzian OPE. This is done constructively, by analytically continuing the 4-point functions using the s-channel OPE expansion in the radial cross-ratios $\rho, \bar{\rho}$. We prove a key fact that $|\rho|,|\bar{\rho}|<1$ inside the forward tube, and set bounds on how fast $|\rho|,|\bar{\rho}|$ may tend to 1 when approaching the Minkowski space.

We also provide a guide to the axiomatic QFT literature for the modern CFT audience. We review the Wightman and Osterwalder-Schrader (OS) axioms for Lorentzian and Euclidean QFTs, and the celebrated OS theorem connecting them. We also review a classic result of Mack about the distributional OPE convergence. Some of the classic arguments turn out useful in our setup. Others fall short of our needs due to Lorentzian assumptions (Mack) or unverifiable Euclidean assumptions (OS theorem).

Keywords: Conformal and W Symmetry, Conformal Field Theory, Field Theories in Higher Dimensions

ArXiv EPrint: 2104.02090

I wanted to learn about elementary particles by studying boiling water. Alexander Polyakov [1] 


\section{Contents}

1 Introduction $\quad 1$

1.1 Executive summary of results for CFT experts 4

2 Axioms $\quad 7$

$\begin{array}{lll}2.1 & \text { Wightman axioms } & 7\end{array}$

$\begin{array}{ll}2.2 & \text { Osterwalder-Schrader axioms } \\ & 10\end{array}$

$\begin{array}{lll}2.3 & \text { Euclidean CFT axioms } & 14\end{array}$

3 Euclidean CFT $\Rightarrow$ Osterwalder-Schrader $\quad 18$

$\begin{array}{lll}3.1 & \text { OS reflection positivity } & 19\end{array}$

3.2 Denseness and Hilbert space implications $\quad 22$

$\begin{array}{lll}3.3 & \text { OS clustering } & 23\end{array}$

4 Euclidean CFT $\Rightarrow$ Wightman: basic strategy $\quad 24$

4.1 Recovering Minkowski averages from Euclidean averages 28

5 Two- and 3-point functions $\quad 31$

5.1 Comparison with the $i \varepsilon$-prescription 33

6 Scalar 4-point function $\quad 35$

6.1 Informal introduction to basic issues 35

6.2 Euclidean 4-point function in radial coordinates 37

$\begin{array}{lll}6.3 & \text { Analytic continuation } & 39\end{array}$

6.4 Proof of $z, \bar{z} \notin[1,+\infty)$

6.5 4-point function powerlaw bound $\quad 45$

6.5.1 A powerlaw bound on $\frac{1}{1-r(c)} \quad 46$

6.6 Conformal invariance 50

6.6.1 Conformal invariance in terms of cross ratios 52

$\begin{array}{ll}\text { 6.6.2 Fixing points } & 56\end{array}$

$\begin{array}{lll}6.7 & \text { Wightman positivity } & 57\end{array}$

$\begin{array}{lll}\text { 6.7.1 Wightman states } & 58\end{array}$

$\begin{array}{ll}\text { 6.7.2 OS states for complexified times } & 59\end{array}$

$\begin{array}{lll}6.8 & \text { Wightman clustering } & 60\end{array}$

$\begin{array}{lll}6.8 .1 & 2+2 \text { split } & 60\end{array}$

$\begin{array}{lll}6.8 .2 & 3+1 \text { split } & 60\end{array}$

6.9 Local commutativity 62

6.9.1 Local commutativity for CFT 4-point functions 65

6.10 Generalization to non-identical scalars 66 
7 Optimal powerlaw bound from Cauchy-Schwarz $\rho, \bar{\rho}$ inequality $\quad 68$

7.1 Proof of lemma $7.1 \quad 68$

$\begin{array}{lll}7.2 & \text { Optimal bound for } \rho, \bar{\rho} & 69\end{array}$

$\begin{array}{lll}7.3 & \text { Proof of theorem } 7.2 & 70\end{array}$

8 OPE convergence in the forward tube and in Minkowski space $\quad 73$

$\begin{array}{lll}8.1 & \text { Convergence of conformal block decomposition for 4-point functions } & 73\end{array}$

8.1.1 Convergence rate for compactly supported test functions 75

$\begin{array}{lll}\text { 8.2 OPE convergence in the sense of } \mathcal{H}^{\mathrm{CFT}} & 77\end{array}$

$\begin{array}{ll}\text { 8.3 Comparison to Mack's work on OPE convergence } & 80\end{array}$

8.3.1 Relating Mack's kernel $B$ to the Euclidean OPE kernel $C$

9 Review of Osterwalder-Schrader theorem $\quad 86$

$\begin{array}{lll}9.1 & \text { The argument of [2] } & 87\end{array}$

$\begin{array}{lll}9.2 & \text { The argument of [3] } & 89\end{array}$

$\begin{array}{lll}\text { 9.2.1 } & \text { Fixing the equivalence theorem } & 89\end{array}$

$\begin{array}{ll}\text { 9.2.2 Wightman axioms from linear growth condition } & 90\end{array}$

$\begin{array}{lll}9.2 .3 & \text { Analytic continuation } & 92\end{array}$

$\begin{array}{ll}\text { 9.2.4 Temperedness bound } & 98\end{array}$

$\begin{array}{ll}10 \text { Conclusions } & 100\end{array}$

$\begin{array}{lr}\text { A Lorentzian CFT literature } & 103\end{array}$

$\begin{array}{lll}\text { A.1 Review of Hartman et al. [4] } & 107\end{array}$

B OS axioms for higher-point functions 112

C Details on Vladimirov's theorem $\quad \mathbf{1 1 5}$

$\begin{array}{lll}\text { C.1 Limit in the sense of distributions } & 115\end{array}$

$\begin{array}{lll}\text { C.2 Proof of theorem } 4.1 & 116\end{array}$

$\begin{array}{lr}\text { D Intuition about lemma } 4.2 & 120\end{array}$

\section{Introduction}

Quantum Field Theory (QFT) can be studied via constructive or axiomatic approaches. The former use microscopic formulations, while the latter rely on axioms. There are many constructive approaches, e.g. using Hamiltonian, path integral, lattice, etc. There are also many axiomatic approaches, corresponding to various sets of axioms (Wightman [5], Osterwalder-Schrader [2, 3], Haag-Kastler [6, 7], etc.). Historically, axiomatic approaches played an important role in clarifying general QFT properties, but they did not have a 
tremendous success in making predictions about concrete theories in $d>2$ dimensions. $^{1}$ This started to change recently, with the revival of the bootstrap philosophy [10]. Our focus here will be on conformal field theories (CFTs) in dimension $d \geqslant 2$, i.e. QFTs invariant under the action of conformal group, which are nowadays studied via the conformal bootstrap. This axiomatic approach led to precise determinations of many experimentally measurable quantities, such as the critical exponents of the $3 \mathrm{~d}$ Ising [11-15], $O(N)$ [15-19] and other critical points (see review $[20]$ ). ${ }^{2}$

The numerical conformal bootstrap relies on the "Euclidean CFT axioms", ${ }^{3}$ which specify properties of correlation functions in any unitary CFT in $\mathbb{R}^{d}$ via a set of simple and commonly accepted rules, such as the unitarity bounds on primary operator dimensions, conformally invariant and convergent Operator Product Expansion (OPE), and reality constraints on OPE coefficients.

On the other hand, correlation functions in a general unitary QFT (and in particular in a CFT) should satisfy Osterwalder-Schrader (OS) and Wightman axioms. It is then interesting and important to inquire what is the relation of Euclidean CFT axioms to these other sets of axioms. ${ }^{4}$ To carry out this analysis will be the main goal of our paper. Our main conclusion will be that the Euclidean CFT axioms imply OS axioms and Wightman axioms for 2,3 and 4-point functions. In this paper we only consider bosonic operators.

The relation of Euclidean CFT and OS axioms is perhaps not so surprising since they both deal with the Euclidean correlation functions. It is more interesting that we are able to construct Minkowski $n$-point functions (for $n=2,3,4$ ), and show that they satisfy Wightman axioms, such as temperedness, spectral condition, and unitarity. Temperedness (being a tempered distribution) is a crucial property of Minkowski correlation functions: it shows that in a certain averaged sense they are meaningful everywhere including the light-cone and double light-cone singularities. One might be tempted to think that in CFT this question is relatively trivial due to OPE. However, as discussed in [36, 37], already for 4-point functions there exist causal configurations of points in Minkowski space, away from the null cones, for which no OPE channel is convergent in the conventional sense. We briefly discuss one such example in the conclusions (section 10).

A theorem of Osterwalder and Schrader [2,3] says that, under some extra assumption, OS axioms imply Wightman axioms. Unfortunately this extra assumption, the so called "linear growth condition", which involves the Euclidean $n$-point functions with arbitrarily high $n$, appears impossible to verify from the Euclidean CFT axioms (see section 9). For this reason we cannot appeal to the OS theorem, and we will give an independent derivation of Wightman axioms for CFT correlators.

The study of distributional properties of CFT correlators started in our recent work [36]. There, we considered expansions of the CFT 4-point function $g(\rho, \bar{\rho})$ in terms of

\footnotetext{
${ }^{1}$ In $d=2$ significant progress has been achieved axiomatically for massive integrable models using the S-matrix bootstrap [8] as well as for rational CFTs [9].

${ }^{2}$ There is also an ongoing revival of the S-matrix bootstrap applicable to nonintegrable massive QFTs in $d \geq 2[21-35]$.

${ }^{3}$ The term "Euclidean bootstrap axioms" is also sometimes used.

${ }^{4}$ Clarifying the relation to the Haag-Kastler axioms appears more challenging as those axioms do not deal with correlation functions but with operator algebras.
} 
conformally invariant cross-ratios $\rho, \bar{\rho}$. While such expansions converge in the usual sense for $|\rho|,|\bar{\rho}|<1$, in [36] we showed that they also converge for $|\rho|,|\bar{\rho}|=1$ in the sense of distributions. As explained in [36], results of this sort follow naturally from the theory of functions of several complex variables (namely Vladimirov's theorem), given some apriori information about the growth of the analytically continued correlator. That key insight of [36], "Look for a powerlaw bound!", will be transported here from the cross-ratio to the position space.

The readers interested in our main technical result - analytic continuation of a scalar Euclidean CFT 4-point function to the forward tube and showing that the Minkowski 4point function is a tempered distribution - may follow the fast track: start with the executive summary in section 1.1, proceed to sections 4 and 5 (skipping 4.1 and 5.1), then continue with sections 6.1-6.5 (optionally including section 7) and finish with the discussion in section 10 . This is only about $20-25$ pages.

On the other hand, we made an effort to make the exposition self-contained and to review main ideas and results of the axiomatic quantum field literature, directly or tangentially related to our discussion. This explains the great total length of our work. The reader will find here:

- A review of classic QFT axioms: Wightman (section 2.1), OS (section 2.2). A review of main implications among these axioms: how OS reflection positivity robustly implies Wightman positivity (section 6.7). A review of the Osterwalder-Schrader theorem about how OS axioms imply Wightman axioms under the additional assumptions of the linear growth condition (section 9).

- A formulation of 'Euclidean CFT axioms' for unitary CFT in Euclidean space $\mathbb{R}^{d}$ (section 2.3). We consider bosonic fields in arbitrary tensor representations. Our axioms encode in a consistent and non-redundant manner the main properties used in the numerical conformal bootstrap calculations. ${ }^{5}$ They are applicable to any globally conformally invariant theory in $d \geqslant 2$. We do not include the axioms involving the local stress tensor and the conserved currents. In particular our axioms would be too weak (but valid) when applied to local $2 \mathrm{~d}$ CFTs, as they know nothing about the Virasoro algebra. ${ }^{6}$ See remark 2.6 for a comparison between our axioms and the CFT rules gathered in the conformal bootstrap review [20].

- A derivation of OS axioms from Euclidean CFT axioms for 4-point function (section 3). A notable result is a rigorous proof that the state produced by two operators in lower Euclidean half-space belongs to the CFT Hilbert space generated by singlepoint operator insertions. The higher-point case is discussed in appendix B, where we need a somewhat stronger form of the OPE axiom than in section 2.3.

\footnotetext{
${ }^{5}$ See also [38] for a recent informal exposition of Euclidean CFT axioms (incomplete as it omits tensor fields) for mathematical physics audience. Ref. [39] attempted the axiomatization of Euclidean CFT in $d>2$ dimensions similar to Segal's axioms in $d=2$ [40]. It is not immediately obvious if the axioms of ref. [39] are equivalent to ours, or how to connect them to practical CFT calculations.

${ }^{6}$ Recall that while in $d=2$ assuming the existence of a local stress tensor immediately implies Virasoro symmetry, no such dramatic statements are currently known in $d>2$.
} 
- A derivation of Wightman axioms from Euclidean CFT axioms for scalar 4-point functions (section 6). As mentioned above, this is the main technical result of the paper. The key observation is that the analytic continuation from Euclidean to Minkowski can be done in a way which keeps the s-channel $\rho, \bar{\rho}$ less than 1 in absolute value along the continuation contour. When we take the Minkowski limit $|\rho|,|\bar{\rho}|$ stay less than 1 for some causal orderings and approaches 1 for others (see [37] for a classification) but even if $|\rho|,|\bar{\rho}| \rightarrow 1$ they approach this limit sufficiently slowly ("powerlaw bound") which guarantees that the Minkowski 4-point function is a tempered distribution everywhere. E.g. using this argument we can show for the first time that the CFT 4-point function is a tempered distribution on the double light-cone singularity.

- We include also a derivation of other expected properties of Minkowski 4-point functions, such as conformal invariance, unitarity, clustering, and local commutativity (sections 6.6-6.9). The reader may find it curious how some of the steps do not use conformal invariance at all but follow simply from analyticity and/or OS positivity.

- Section 7 proves a curious geometric "Cauchy-Schwarz" inequality for $\rho, \bar{\rho}$ variables which provides an alternative way of understanding why $|\rho|,|\bar{\rho}|<1$ in the forward tube. It bounds $\rho, \bar{\rho}$ for a generic configuration by $\rho, \bar{\rho}$ for reflection-symmetric configurations. It would be interesting to find an elementary proof of this inequality (our proof uses some facts about conformal blocks).

- Section 8 shows that the (s-channel) conformal block expansion of 4-point Wightman functions converges in the sense of distributions for all configurations of points in Minkowski space. It is also shown that the OPE for the state-valued distributions $\left|\mathcal{O}\left(x_{1}\right) \mathcal{O}\left(x_{2}\right)\right\rangle$ with $x_{1}, x_{2} \in \mathbb{R}^{d-1,1}$ converges in the sense of distributions. We discuss the relationship of these results to the classic work of Mack [41] and prove estimates for the convergence rates of these expansions.

- Section 9 contains a review of the papers $[2,3]$ by Osterwalder and Schrader. In particular, we discuss the gap in the arguments of [2] which precludes the derivation of Wightman axioms from the OS axioms of [2], and explain in detail how this gap is filled in [3] with the addition of new axioms.

- Appendix A is a guide to the modern Lorentzian CFT literature: conformal collider bounds, light-cone bootstrap, causality constraints, the Lorentzian OPE inversion formula, light-ray operators, etc. Our results help put some of these considerations on a firmer footing. We indicate the most critical remaining steps, which still wait to be rigorously derived from the Euclidean CFT axioms.

We conclude in section 10. Some additional technical details are given in appendices B)-(D.

\subsection{Executive summary of results for CFT experts}

This paper is rather lengthy as a result of our attempt to make it self-contained. In this section we give a brief summary of the main technical results, aimed at the more expert 
readers who may not wish to read the expository parts of this paper. Note, however, that here we omit many secondary results, some of which are mentioned above.

The basic question we address in this paper is the question of the distributional properties of Wightman 4-point functions in CFTs. As is well-known, Wightman n-point distributions are recovered from the boundary values of functions holomorphic in the forward tube $\mathcal{T}_{n}$. For an $n$-point function

$$
\left\langle 0\left|\mathcal{O}_{1}\left(x_{1}\right) \cdots \mathcal{O}_{n}\left(x_{n}\right)\right| 0\right\rangle
$$

the forward tube is defined as the set of $x_{i} \in \mathbb{C}^{1, d-1}$ subject to

$$
\operatorname{Im} x_{1} \prec \operatorname{Im} x_{2} \prec \cdots \prec \operatorname{Im} x_{n},
$$

where $\prec$ denotes the causal ordering in $\mathbb{R}^{1, d-1}$. Analyticity in $\mathcal{T}_{n}$ and existence of the boundary value as $\operatorname{Im} x_{i} \rightarrow 0$ is usually derived from Wightman or OS axioms (with extra assumptions in the latter case). In this paper we want to understand this question from the point of view of CFT axioms.

With the cases $n=2,3$ being relatively trivial in a CFT, our main observation is that a particular OPE channel for 4-point functions converges everywhere in the interior of $\mathcal{T}_{4}$. Specifically, we take the $\mathrm{OPE} \mathcal{O}\left(x_{1}\right) \times \mathcal{O}\left(x_{2}\right)$ in the Wightman function of identical scalar operators

$$
\left\langle 0\left|\mathcal{O}\left(x_{1}\right) \mathcal{O}\left(x_{2}\right) \mathcal{O}\left(x_{3}\right) \mathcal{O}\left(x_{4}\right)\right| 0\right\rangle .
$$

This OPE is expected to converge, at least distributionally for real $x_{i}$, from the results of Mack [41]. However, his work assumes Wightman axioms from the beginning, and our goal here is to clarify the implications of Euclidean CFT axioms, which only assume convergence of the Euclidean OPE.

To see that this OPE channel converges, we show in lemma 6.1 that for any configuration of $x_{i}$ in $\mathcal{T}_{4}$ the radial cross-ratios $\rho$ and $\bar{\rho}$ for this OPE belong to the open unit disk (for the definition of radial cross-ratios, see section 6.2):

$$
|\rho|,|\bar{\rho}|<1 .
$$

This implies convergence of the conformal block expansion in $\mathcal{O}\left(x_{1}\right) \times \mathcal{O}\left(x_{2}\right)$ channel in the interior of $\mathcal{T}_{4}$. A technical way to see this is to note that the expansion in descendants

$$
g(\rho, \bar{\rho})=\sum_{h, \bar{h}>0} p_{h, \bar{h}} \rho^{h} \bar{\rho}^{\bar{h}},
$$

where $g$ is the conformally-invariant part of the 4 -point function and $p_{h, \bar{h}}>0$, can be bounded term-by-term by

$$
\left|p_{h, \bar{h}} \rho^{h} \bar{\rho}^{\bar{h}}\right| \leqslant p_{h, \bar{h}} r^{h} r^{\bar{h}},
$$

where $r=\max (|\rho|,|\bar{\rho}|)<1$. The right-hand side of this inequality is a term in the expansion of $g(r, r)$, a Euclidean configuration in which the OPE is known to converge, so (1.5) is dominated by a convergent series. Therefore, (1.5) is convergent for $r<1$, and moreover uniformly so on compact subsets, since each term $p_{h, \bar{h}} r^{h} r^{\bar{h}}$ is monotonic. We can then conclude that the sum $g(\rho, \bar{\rho})$ is a holomorphic function. 
This reasoning also gives us the inequality

$$
|g(\rho, \bar{\rho})| \leqslant g(r, r) .
$$

So, we find that the correlator can be recovered inside of $\mathcal{T}_{4}$ from the $\mathcal{O}\left(x_{1}\right) \times \mathcal{O}\left(x_{2}\right)$ OPE, is analytic there, and is bounded by a Euclidean configuration.

In section 6.5.1 we establish a stronger form of lemma 6.1, schematically,

$$
1-r(c) \geqslant \operatorname{dist}\left(c, \partial \mathcal{T}_{4}\right)^{a}
$$

for some $a>0$, where $c \in \mathcal{T}_{4}$ is a configuration of 4 points in $\mathcal{T}_{4}$. (The more precise form also bounds $1-r(c)$ as $c$ goes to infinity.) This immediately implies a powerlaw bound on $g(r, r)$ near the boundary of $\mathcal{T}_{4}$. Indeed, near $r \rightarrow 1$ the correlator is dominated by the identity operator in the crossed channel, and so

$$
g(r(c), r(c)) \leqslant C(1-r(c))^{-4 \Delta_{\varphi}}
$$

and thus

$$
|g(c)| \leqslant C \operatorname{dist}\left(c, \partial \mathcal{T}_{4}\right)^{b}
$$

for some real $b$. This allows us to use Vladimirov's theorem 4.1, which implies that the boundary limit (as $x_{i}$ approach real Minkowski values) of (1.3) exists in the space of tempered distribution. (We establish a more refined bound for $x_{i} \rightarrow \infty$ to claim temperedness.)

The above bounds hold just as well for the truncated conformal block expansion as for the full correlator. A variant of theorem 4.1 then allows us to conclude that the conformal block expansion, while converging in the sense of functions in the interior of $\mathcal{T}_{4}$, converges in the space of tempered distributions on the Minkowski boundary.

We extend the above results to correlators of non-identical scalars by replacing the term-by-term bound (1.6) with a standard Cauchy-Schwarz argument, bounding the correlator in terms of a product of two reflection-symmetric Euclidean correlators. While it is intuitively obvious that similar arguments should also work for operators with spin, we found that the extension to spinning operators, due to the complexity of tensor structures, requires enough additional work to warrant a separate paper [42].

Finally, in section 7 we prove theorem 7.2 , which gives an optimal bound of the form (1.8). Specifically, it is

$$
r(c)^{2} \leqslant r\left(c_{12}\right) r\left(c_{34}\right)
$$

together with a bound for the right-hand side. Here, if $c=\left(x_{1}, x_{2}, x_{3}, x_{4}\right)$ (where $x_{i}$ are real in Minkowski space), then $c_{12} \equiv\left(x_{1}, x_{2}, x_{2}^{*}, x_{1}^{*}\right)$ and $c_{34} \equiv\left(x_{4}^{*}, x_{3}^{*}, x_{3}, x_{4}\right)$. The bound for the right-hand side is easier to obtain because the configurations $c_{12}$ and $c_{34}$ are reflection symmetric. This is done in section 7.2. The bound (1.11) looks like a Cauchy-Schwarz-type inequality, and is indeed derived from the Cauchy-Schwarz inequality for unitary conformal blocks (7.21). The latter is true because of the unitarity of conformal representations corresponding to these blocks. In the limit $\Delta+\ell \rightarrow \infty, \Delta-\ell$ fixed, conformal blocks are dominated by $r^{(\Delta+\ell) / 2}$, which reduces the conformal block Cauchy-Schwarz inequality to $(1.11)$. 


\section{Axioms}

\subsection{Wightman axioms}

In this section we will state the properties of Wightman correlation functions in a unitary QFT, to which we will refer here as "Wightman axioms." These axioms appear as "properties of the vacuum expectation values" in [5], section 3-3, and as (W1)-(W5) in [43], theorem II.1. Refs. [5, 43] give in addition another set of axioms (called Gårding-Wightman axioms in [43]) saying that fields are operator-valued distributions in the Hilbert space on which the Lorentz group acts, etc. This other set of axioms will not be used in this work. In any case, the Wightman reconstruction theorem [5] says that the two sets of axioms are equivalent.

A unitary QFT in Minkowski space studies n-point correlators

$$
\left\langle\varphi_{1}\left(x_{1}\right) \ldots \varphi_{n}\left(x_{n}\right)\right\rangle
$$

(Wightman functions) of local operators $\varphi_{i}(x), x \in \mathbb{R}^{1, d-1} \cdot{ }^{7}$ For simplicity in this paper we will only consider bosonic operators, although more generally one should allow fermionic operators and spinor representations. Wightman functions are translation and $\mathrm{SO}(1, d-$ 1) invariant. ${ }^{8}$ We will choose a basis of local operators $\mathcal{O}_{i}$ transforming in irreducible $\mathrm{SO}(1, d-1)$ representations $\rho_{i}$. Then, Wightman functions remain invariant when

$$
\mathcal{O}_{i}^{\alpha}(x) \rightarrow \rho_{i}(g)^{\alpha}{ }_{\beta} \mathcal{O}_{i}^{\beta}\left(g^{-1} x\right),
$$

where $g \in \mathrm{SO}(1, d-1)$, and $\rho_{i}(g)$ are finite-dimensional matrices of the representation $\rho_{i}\left(\alpha, \beta=1 \ldots \operatorname{dim} \rho_{i}\right)$. Let $\mathcal{C}$ be the complex vector space whose elements are arbitrary components of $\mathcal{O}_{i}$ 's, and their finite linear combinations with constant complex coefficients. Operators $\varphi_{i}$ in (2.1) can be arbitrary elements of $\mathcal{C}$, and Wightman function (2.1) is multilinear in $\varphi_{i}$. Note that in this and the next section derivatives of local operators (of any order) are counted as independent operators, while in the CFT section 2.3 we will start making distinction between primaries and their derivatives.

Wightman functions (2.1) are required to be tempered distributions, i.e. can be paired with Schwartz class test functions $f\left(x_{1}, \ldots, x_{n}\right)$. For this reason they are sometimes referred to as "Wightman distributions". Note that the test functions $f\left(x_{1}, \ldots, x_{n}\right)$ with which Wightman functions are paired do not have to vanish at coincident points (unlike for the Schwinger functions discussion in the next section). This means that, in a distributional sense, Wightman functions have meaning for all configurations, including coincident points and light-cone singularities. Translation and Lorentz invariance of Wightman functions are

\footnotetext{
${ }^{7}$ In this paper, we use the term "operator" according to CFT terminology, where the term "field" is used in Wightman and Osterwalder-Schrader terminology. For example, in refs. [5, 43], the term "operator" is reserved for the actual unbounded Hilbert space operator $\phi(f)$ defined on a dense domain of the Hilbert space (which justifies the terminology "operator-valued distribution" $\phi$ in the Gårding-Wightman formulation).

${ }^{8}$ Here and everywhere in this paper, $\mathrm{SO}(1, d-1)$ means the connected component of the group. E.g. $\operatorname{diag}(-1,-1,1, \ldots, 1)$ is not included. Of course, it is possible for the theory to also possess discrete spacetime symmetries such as time inversion or space reflection. The consequences of such symmetries are straightforward to figure out and are not discussed in this paper.
} 
also understood not pointwise but in the sense of distributions (i.e. that the pairing should remain invariant if the test function is transformed in the dual way). ${ }^{9}$

We will not consider here other Minkowski correlators, such as retarded, advanced, or time ordered, which are obtained from Wightman functions multiplying by thetafunctions of time coordinate differences, and whose distributional properties require a separate discussion.

Limiting to the bosonic case as we are, local commutativity (also called microcausality) morally says that operators commute at spacelike separation. Wightman axioms impose this as a constraint on Wightman functions:

$$
\left\langle\varphi_{1}\left(x_{1}\right) \ldots \varphi_{p}\left(x_{p}\right) \varphi_{p+1}\left(x_{p+1}\right) \ldots \varphi_{n}\left(x_{n}\right)\right\rangle=\left\langle\varphi_{1}\left(x_{1}\right) \ldots \varphi_{p+1}\left(x_{p+1}\right) \varphi_{p}\left(x_{p}\right) \ldots \varphi_{n}\left(x_{n}\right)\right\rangle
$$

whenever $x_{p}-x_{p+1}$ is spacelike: $\left(x_{p}-x_{p+1}\right)^{2}>0$ (in this paper we use the $-+\ldots+$ convention). Since we are talking about distributions, this constraint means that (2.3) holds when paired with any test function whose support is contained in $\left(x_{p}-x_{p+1}\right)^{2}>0$.

Clustering says that correlators should factorize if two groups of points are far separated in a spacelike direction:

$$
\begin{aligned}
\left\langle\varphi_{1}\left(x_{1}\right) \ldots \varphi_{p}\left(x_{p}\right) \varphi_{p+1}\left(x_{p+1}+\lambda a\right) \ldots \varphi_{n}\left(x_{n}+\lambda a\right)\right\rangle & \\
& \rightarrow\left\langle\varphi_{1}\left(x_{1}\right) \ldots \varphi_{p}\left(x_{p}\right)\right\rangle\left\langle\varphi_{p+1}\left(x_{p+1}\right) \ldots \varphi_{n}\left(x_{n}\right)\right\rangle
\end{aligned}
$$

as $\lambda \rightarrow \infty$ for any spacelike vector $a$, limit understood in the sense of distributions.

We next discuss the spectral condition. By translation invariance we can write

$$
\left\langle\varphi_{1}\left(x_{1}\right) \ldots \varphi_{n}\left(x_{n}\right)\right\rangle=W\left(\xi_{1}, \ldots, \xi_{n-1}\right), \quad \xi_{k}=x_{k}-x_{k+1},
$$

where $W$ is a tempered distribution in one less variable. Consider its Fourier transform:

$$
\widehat{W}\left(q_{1}, \ldots, q_{n-1}\right)=\int W\left(\xi_{1}, \ldots, \xi_{n-1}\right) e^{i \sum_{k=1}^{n-1} q_{k} \cdot \xi_{k}} d \xi_{1} \ldots d \xi_{n},
$$

where $q_{k}=\left(E_{k}, \mathbf{q}_{k}\right), \xi_{k}=\left(t_{k}, \xi_{k}\right), q_{k} \cdot \xi_{k}=-E_{k} t_{k}+\mathbf{q}_{k} \cdot \xi_{k}$. Since $W$ is a tempered distribution, the Fourier transform $\widehat{W}$ is well defined and is also a tempered distribution. The spectral condition then says that $\widehat{W}$ must be supported in the product of closed forward light cones, i.e. in the region

$$
E_{k} \geqslant \mathbf{q}_{k}, \quad k=1,2, \ldots, n-1 .
$$

For the two remaining conditions we need to discuss conjugation. Physically, each operator $\varphi$ should have a conjugate $\varphi^{\dagger}$. In the discussed framework we cannot define $\varphi^{\dagger}$ as an adjoint of an operator acting on a Hilbert space, since we do not have a Hilbert space. Instead, we will simply assume that there is a rule which associates $\varphi^{\dagger}$ to $\varphi$, and impose the expected relations at the level of correlation functions (eq. (2.10) below). This rule, conjugation

\footnotetext{
${ }^{9}$ Although Wightman functions can be shown to be real-analytic at some totally spacelike-separated configurations (Jost points), in general they may be singular even away from light cones (in particular when there are timelike separations).
} 
map $\dagger: \mathcal{C} \rightarrow \mathcal{C}$, associates to each independent component $\mathcal{O}_{i}^{\alpha}\left(\alpha=1 \ldots \operatorname{dim} \rho_{i}\right)$ of the above-mentioned basis of $\mathcal{C}$ a conjugate operator $\left(\mathcal{O}_{i}^{\alpha}\right)^{\dagger}$. This map is required to be an involution, i.e. $\dagger \dagger=1$. Furthermore, it is extended to the whole of $\mathcal{C}$ by anti-linearity, i.e. $\left(c_{1} \varphi_{1}+c_{2} \varphi_{2}\right)^{\dagger}=c_{1}^{*} \varphi_{1}^{\dagger}+c_{2}^{*} \varphi_{2}^{\dagger} \cdot{ }^{10}$

Let us group operators $\left(\mathcal{O}_{i}^{\alpha}\right)^{\dagger}$ in a multiplet which we denote by $\mathcal{O}_{i}^{\dagger}$, i.e. $\left(\mathcal{O}_{i}^{\dagger}\right)^{\alpha}=\left(\mathcal{O}_{i}^{\alpha}\right)^{\dagger}$. We will see below that $\left(\mathcal{O}_{i}^{\dagger}\right)^{\alpha}$ transform under $g \in \mathrm{SO}(1, d-1)$ with matrices complexconjugate to those of $\mathcal{O}_{i}^{\alpha}$ :

$$
\mathcal{O}_{i}^{\alpha} \rightarrow \rho_{i}(g)^{\alpha}{ }_{\beta} \mathcal{O}_{i}^{\beta} \Rightarrow\left(\mathcal{O}_{i}^{\dagger}\right)^{\alpha} \rightarrow \overline{\rho_{i}(g)^{\alpha}}\left(\mathcal{O}_{i}^{\dagger}\right)^{\beta} .
$$

In other words, $\mathcal{O}_{i}^{\dagger}$ transforms in the conjugate representation $\overline{\rho_{i}}$.

Since we are considering only bosonic operators, the relevant representations $\rho_{i}$ are tensors $T^{\mu_{1} \ldots \mu_{l}}$, on which $g \in \mathrm{SO}(1, d-1)$ act as:

$$
T^{\mu_{1} \ldots \mu_{l}} \rightarrow\left(\rho_{i}(g) T\right)^{\mu_{1} \ldots \mu_{l}}=g_{\nu_{1}}^{\mu_{1}} \ldots g_{\nu_{l}}^{\mu_{l}} T^{\nu_{1} \ldots \nu_{l}} .
$$

Depending on $\rho_{i}$, these tensors have some fixed rank and mixed symmetry properties. In addition, in even $d$, for tensors with $d / 2$ antisymmetric indices, (anti-)chirality ${ }^{11}$ constraints must be imposed. All tensor representations of $\mathrm{SO}(1, d-1)$ are real (i.e. matrices $\rho_{i}(g)_{\beta}^{\alpha}$ in (2.8) can be chosen real), except for (anti-)chiral representations in $d=0 \bmod 4$ which are complex-conjugate to each other. For operators in real representations we can choose a basis such that $\mathcal{O}_{i}=\mathcal{O}_{i}^{\dagger}$.

After this intermezzo we are ready to formulate hermiticity and positivity conditions. Hermiticity says that complex conjugate correlators equal correlators of conjugated operators in inverted order:

$$
\overline{\left\langle\varphi_{1}\left(x_{1}\right) \ldots \varphi_{n}\left(x_{n}\right)\right\rangle}=\left\langle\varphi_{n}^{\dagger}\left(x_{n}\right) \ldots \varphi_{1}^{\dagger}\left(x_{1}\right)\right\rangle .
$$

This would be true of course if $\varphi$ 's were operators acting on a Hilbert space, with $\varphi^{\dagger}$ 's their adjoints. In the present framework without Hilbert space it is imposed as an axiom. This axiom implies in particular (2.8), i.e. that $\mathcal{O}_{i}^{\dagger}$ transforms in the conjugate irrep $\overline{\rho_{i}}$.

The last Wightman axiom, positivity, is most conveniently written down using the language of states. One considers basic ket states $\left|\psi\left(f, \varphi_{1}, \ldots, \varphi_{n}\right)\right\rangle$, associated with $n$ local operators $\varphi_{1}, \ldots, \varphi_{n} \in V$ and a complex Schwartz test function of $n$ variables $f$. One defines the inner product on basic ket states by

$$
\begin{aligned}
\left\langle\psi\left(g, \chi_{1}, \ldots, \chi_{m}\right) \mid \psi\left(f, \varphi_{1}, \ldots, \varphi_{n}\right)\right\rangle:=\int d x d y & \overline{g\left(x_{1}, \ldots, x_{m}\right)} f\left(y_{1}, \ldots, y_{n}\right) \\
& \times\left\langle\chi_{m}^{\dagger}\left(x_{m}\right) \ldots \chi_{1}^{\dagger}\left(x_{1}\right) \varphi_{1}\left(y_{1}\right) \ldots \varphi_{n}\left(y_{n}\right)\right\rangle .
\end{aligned}
$$

The vector space of ket states $\mathcal{H}_{0}$ consists of finite linear combinations $|\Psi\rangle$ of basic ket states, with the inner product extended to it by (anti)linearity. Positivity then says that the so defined inner product is positive semidefinite:

\footnotetext{
${ }^{10}$ The $\dagger$ operation is denoted by $*$ in $[5]$.

${ }^{11}$ Chiral and anti-chiral representations are sometimes also called "self-dual" and "anti-self-dual". We use "chiral" and "anti-chiral" to avoid the clash with "dual representation" in mathematician's sense.
}$$
\langle\Psi \mid \Psi\rangle \geqslant 0 \quad \forall|\Psi\rangle \in \mathcal{H}_{0} .
$$ 
Remark 2.1. A comment is in order concerning the meaning of these states. They may be seen as just a convenient notation, since eq. (2.12) can be rewritten without ever using the word "state" (see [5], eq. (3-35)). But they are more than that: the vector space of states $\mathcal{H}_{0}$ is "almost" the Hilbert space $\mathcal{H}$ of our QFT. The only difference between $\mathcal{H}_{0}$ and $\mathcal{H}$ is that $\mathcal{H}_{0}$ is not complete and may contain some states of zero norm. However, since $\mathcal{H}_{0}$ has a positive semidefinite inner product, as expressed by eq. (2.12), we can obtain from it a Hilbert space $\mathcal{H}$ via a standard procedure of completion and modding out by states of zero norm. This is the first step of the Wightman reconstruction theorem [5], and the resulting Hilbert space $\mathcal{H}$ turns out to be (possibly a superselection sector of) the Hilbert space of the QFT, on which fields can then be realized as operator valued distributions.

Remark 2.2. Although we included hermiticity as a separate axiom because of its suggestive form, it can be derived from positivity, considering the states of the form $|\Psi\rangle=\left|\psi\left(f_{0}, 1\right)\right\rangle+\left|\psi\left(f, \varphi_{1}, \ldots, \varphi_{n}\right)\right\rangle$ where $f_{0} \in \mathbb{C}$ and 1 is the unit operator.

Remark 2.3. Another interesting positivity property of Wightman functions is called Rindler Reflection positivity, or Wedge Reflection positivity [44]. A restricted version of this property (with wedge-ordered points) can be derived from Wightman axioms, while a stronger version (no wedge-ordering) follows from Tomita-Takesaki theory which relies on Haag-Kastler axioms [44]. In CFT context this property has been discussed, e.g., in [45]. We will not discuss these properties in this paper. However, it would be interesting to see whether the stronger form of Rindler positivity (including distributional information) can be derived from CFT axioms without the appeal to Tomita-Takesaki theory (the weaker version following from our results on Wightman axioms and [44]). We believe this can be done, and it could be a nice exercise for someone wishing to master our techniques.

\subsection{Osterwalder-Schrader axioms}

We next describe a version of the Osterwalder and Schrader axioms [2,3] of Euclidean unitary QFT (see the end of the section about the relation to the original OS axioms). The setup is similar to Wightman axioms with $\mathrm{SO}(d)$ replacing $\mathrm{SO}(1, d-1)$. We consider a basis of local bosonic operators $\mathcal{O}_{i}$ transforming ${ }^{12}$ in $\mathrm{SO}(d)$ irreps $\rho_{i}$, counting derivatives as independent operators. Finite linear combinations of their components span a complex vector space $\mathcal{C}$ of local operators. The axioms specify properties of translation and $\mathrm{SO}(d)$ invariant $n$-point correlators (often called Schwinger functions)

$$
\left\langle\varphi_{1}\left(x_{1}\right) \ldots \varphi_{n}\left(x_{n}\right)\right\rangle, \quad \varphi_{i} \in \mathcal{C}, \quad x_{i} \in \mathbb{R}^{d} .
$$

These correlators are defined away from coincident points (i.e. whenever $x_{i} \neq x_{j}$ for each $i, j)$. We will assume that ${ }^{13}$

correlators are real-analytic,

\footnotetext{
${ }^{12}$ In the sense of eq. (2.2) where now $g \in \mathrm{SO}(d)$.

${ }^{13}$ Recall that a $C^{\infty}$ function of $m$ real variables is called real-analytic in a domain $D \subset \mathbb{R}^{m}$ if it has a convergent Taylor series expansion in a small ball around every point of this domain. Equivalently, such a function has an analytic extension to a small open neighborhood of this domain inside $\mathbb{C}^{m}$.
} 
and grow not faster than some power when some points approach each other or go to infinity, i.e.

$$
\left|\left\langle\varphi_{1}\left(x_{1}\right) \ldots \varphi_{n}\left(x_{n}\right)\right\rangle\right| \leqslant C\left(1+\max _{i \neq j}\left(\frac{1}{\left|x_{i}-x_{j}\right|},\left|x_{i}-x_{j}\right|\right)\right)^{p}
$$

with some correlator-dependent positive constants $C, p$. Unlike Wightman axioms, OS axioms do not bother what happens precisely at coincident points (not even in the sense of distributions).

As we are limiting to the bosonic case, correlators remain invariant when operators are permuted: ${ }^{14}$

$$
\left\langle\varphi_{1}\left(x_{1}\right) \ldots \varphi_{n}\left(x_{n}\right)\right\rangle=\left\langle\varphi_{\pi(1)}\left(x_{\pi(1)}\right) \ldots \varphi_{\pi(n)}\left(x_{\pi(n)}\right)\right\rangle .
$$

To formulate the Euclidean version of hermiticity and positivity, we will need some simple facts about $\mathrm{SO}(d)$ representations. Abstractly, for any irrep $\rho$ acting $T^{\alpha} \rightarrow \rho(g)^{\alpha}{ }_{\beta} T^{\beta}$, the conjugate representation $\bar{\rho}$ acts with complex conjugate matrices $\overline{\rho(g)^{\alpha}{ }_{\beta}}$. Since $\mathrm{SO}(d)$ is compact, we have $\bar{\rho} \simeq \rho^{*}$, the dual representation. The $\mathrm{SO}(d)$ irreps $\rho$ are again tensors $T^{\mu_{1} \ldots \mu_{l}}$ like in (2.9), of in general mixed symmetry, and with (anti)-chirality constraints if having $d / 2$ antisymmetric indices in even $d$. All of them are real, except for chiral representations in $d=2 \bmod 4$ which are complex-conjugate to the anti-chiral ones. ${ }^{15}$

We will also need the reflected representation $\rho^{R}$ with matrices $\rho^{R}(g)=\rho\left(g^{R}\right)$, where $g \rightarrow g^{R}=\Theta g \Theta, \Theta=\operatorname{diag}(-1,1, \ldots, 1)$, is an automorphism of $\mathrm{SO}(d)$. For tensor representations, we can consider the map

$$
T^{\mu_{1} \ldots \mu_{l}} \rightarrow \Theta_{\nu_{1}}^{\mu_{1}} \ldots \Theta_{\nu_{l}}^{\mu_{l}} T^{\nu_{1} \ldots \nu_{l}},
$$

which preserves rank and mixed symmetry properties. It also maps chiral to anti-chiral tensors in any even $d$. Whenever the representation space is preserved, this map serves as an intertwiner between $\rho^{R}$ and $\rho$. This means that $\rho^{R} \simeq \rho$ for all tensor representations without chirality constraints, while this operation interchanges chiral and antichiral irreps in any even $d .{ }^{16}$

Applying both conjugation and reflection we get the conjugate reflected representation $\bar{\rho}^{R}$ (isomorphic to dual reflected). From the above it follows that $\bar{\rho}^{R} \simeq \rho$ for all $\mathrm{SO}(d)$ irrreps, except for (anti-)chiral tensors in $d=0 \bmod 4$ which are interchanged.

Just as for Wightman axioms, we will need a conjugation operation $\dagger: \mathcal{C} \rightarrow \mathcal{C}$ on the vector space of local operators, which is involutive, anti-linear, and associates to each independent component $\mathcal{O}_{i}^{\alpha}\left(\alpha=1 \ldots \operatorname{dim} \rho_{i}\right)$ a conjugate operator $\left(\mathcal{O}_{i}^{\dagger}\right)^{\alpha}:=\left(\mathcal{O}_{i}^{\alpha}\right)^{\dagger}$. Then the hermiticity axiom takes the form ${ }^{17}$

$$
\overline{\left\langle\varphi_{1}\left(x_{1}\right) \ldots \varphi_{n}\left(x_{n}\right)\right\rangle}=\left\langle\varphi_{n}^{\dagger}\left(x_{n}^{\theta}\right) \ldots \varphi_{1}^{\dagger}\left(x_{1}^{\theta}\right)\right\rangle,
$$

\footnotetext{
${ }^{14}$ In particular one can sort all operators so that the Euclidean time coordinates are ordered $x_{1}^{0} \geqslant x_{2}^{0} \geqslant$ $\cdots \geqslant x_{n}^{0}$, and Euclidean correlator for any other ordering can be obtained by trivially permuting field labels.

${ }^{15}$ This well-known shift from $d=0 \bmod 4$ for $\mathrm{SO}(1, d-1)$ is induced by raising the indices of the $\varepsilon$-tensor. E.g. $\varepsilon^{01} \varepsilon_{10}=-1$ for $\mathrm{SO}(2)$, while it is 1 for $\mathrm{SO}(1,1)$.

${ }^{16} \mathrm{In}$ odd $d, \Theta$ is a product of -1 and an $\mathrm{SO}(d)$ matrix, so that $g \rightarrow g^{R}$ is an inner automorphism. This provides another argument why $\rho^{R} \simeq \rho$ for all irreducible $\mathrm{SO}(d)$ representations in odd $d$.

${ }^{17}$ Although we write the operators in the r.h.s. in the inverted order like in (2.10), permutation invariance renders this detail unimportant for the OS axioms.
} 
similar to the Minkowski counterpart (2.10) but with an important difference that the operators in the r.h.s. are put at reflected positions

$$
x^{\theta}:=\Theta x .
$$

This change has a consequence that $\mathcal{O}_{i}^{\dagger}$ transforms in the conjugate reflected representation $\bar{\rho}_{i}^{R}$, explaining why we introduced this concept in the first place. ${ }^{18}$ For self-conjugatereflected representations we may choose a basis such that

$$
\left(\mathcal{O}_{i}^{\dagger}\right)^{(\mu)}=\Theta_{(\nu)}^{(\mu)} \mathcal{O}_{i}^{(\nu)}
$$

where $\Theta_{(\nu)}^{(\mu)}:=\Theta_{\nu_{1}}^{\mu_{1}} \ldots \Theta_{\nu_{l}}^{\mu_{l}}$ is the intertwiner $(2.17)$.

To write positivity, basic ket states $\left|\psi\left(f, \varphi_{1}, \ldots, \varphi_{n}\right)\right\rangle$ are associated with $n$ local operators $\varphi_{1}, \ldots, \varphi_{n} \in \mathcal{C}$ and a complex compactly supported Schwartz test function of $n$ variables $f\left(x_{1}, \ldots, x_{n}\right)$ which vanishes unless all points are in the lower half space and have time variables ordered: $0>x_{1}^{0}>x_{2}^{0}>\cdots>x_{n}^{0}$. These support requirements were absent in the Wightman case. The inner product on the basic ket states is defined by

$$
\begin{aligned}
\left\langle\psi\left(g, \chi_{1}, \ldots, \chi_{m}\right) \mid \psi\left(f, \varphi_{1}, \ldots, \varphi_{n}\right)\right\rangle:= & \int d x d y \overline{g\left(y_{1}^{\theta}, \ldots, y_{m}^{\theta}\right)} f\left(x_{1}, \ldots, x_{n}\right) \\
& \times\left\langle\chi_{m}^{\dagger}\left(y_{m}\right) \ldots \chi_{1}^{\dagger}\left(y_{1}\right) \varphi_{1}\left(x_{1}\right) \ldots \varphi_{n}\left(x_{n}\right)\right\rangle,
\end{aligned}
$$

and is extended by (anti)linearity to the vector space $\mathcal{H}_{0}^{\mathrm{OS}}$ of finite linear combinations $|\Psi\rangle$ of basic ket states. In this notation, positivity takes the same form as (2.12), i.e. that the so defined inner product must be positive semidefinite:

$$
\langle\Psi \mid \Psi\rangle \geqslant 0 \quad \forall|\Psi\rangle \in \mathcal{H}_{0}^{\mathrm{OS}} .
$$

This is referred to as "OS reflection positivity" because of the reflected $g$ arguments in (2.21), differently from the Wightman case. Because of this reflection and the above test function support requirements, all operators in (2.21) sit at separated positions. This is one reason why the OS axioms involve ordinary functions, without worrying about coincident points. In contrast, Wightman positivity integrates operator insertions over coincident points and makes sense only for distributions.

Just as in the Wightman case (remark 2.1), we can complete the vector space $\mathcal{H}_{0}^{\mathrm{OS}}$, mod out by states of zero norm, and obtain a Hilbert space $\mathcal{H}^{\mathrm{OS}}$ of the Euclidean theory.

Although we included hermiticity as an independing axiom, it can be derived from positivity, just as in remark 2.2 in the Wightman case.

One simple consequence of OS reflection positivity is pointwise positivity of $2 n$-point functions at reflection invariant configurations of points:

$$
\left\langle\varphi_{n}^{\dagger}\left(x_{n}^{\theta}\right) \ldots \varphi_{1}^{\dagger}\left(x_{1}^{\theta}\right) \varphi_{1}\left(x_{1}\right) \ldots \varphi_{n}\left(x_{n}\right)\right\rangle \geqslant 0
$$

for any $x_{1}, \ldots, x_{n}$ in the lower half space. ${ }^{19}$ This follows from $(2.22)$ by taking $|\Psi\rangle=$ $\left|\psi\left(f, \varphi_{1}, \ldots, \varphi_{n}\right)\right\rangle$ and localizing $f$ near one configuration of points. In general, imposing (2.23) for all $\varphi$ 's and $x$ 's would be clearly weaker than full OS reflection positivity.

\footnotetext{
${ }^{18}$ Indeed we have $\left\langle\left(\mathcal{O}_{i}^{\dagger}\right)^{\alpha}(x) \ldots\right\rangle=\overline{\left\langle\mathcal{O}_{i}^{\alpha}\left(x^{\theta}\right) \ldots\right\rangle}=\overline{\rho(g)^{\alpha}}\left\langle\mathcal{O}_{i}^{\beta}\left(g^{-1} x^{\theta}\right) \ldots\right\rangle=\overline{\rho(g)^{\alpha}}{ }_{\beta}\left\langle\left(\mathcal{O}_{i}^{\dagger}\right)^{\beta}\left(\left(g^{R}\right)^{-1} x\right) \ldots\right\rangle$.

${ }^{19}$ For tensor operator in self-conjugate-reflected representations, choosing the real basis (2.20), this becomes $\left\langle\ldots \Theta_{(\nu)}^{(\mu)} \mathcal{O}^{(\nu)}\left(x^{\theta}\right) \mathcal{O}^{(\mu)}(x) \ldots\right\rangle \geqslant 0$ (no sum on $\mu$ ), i.e. tensor indices are also reflected.
} 
E.g. (2.22), but not (2.23), can be used to bound 3-point functions in terms of 2- and 4-point functions, or non-reflection-invariant 4-point functions by reflection-invariant ones. However for CFTs we will see below that OS reflection positivity can be reduced to a form of (2.23) for 2-point functions plus a form of (2.18) for 3-point functions.

Finally, the OS clustering asserts that

$$
\begin{gathered}
\lim _{\lambda \rightarrow \infty} \int d x d y \overline{g\left(y_{1}^{\theta}, \ldots, y_{m}^{\theta}\right)} f\left(x_{1}, \ldots, x_{n}\right)\left\langle\chi_{m}^{\dagger}\left(y_{m}\right) \ldots \chi_{1}^{\dagger}\left(y_{1}\right) \varphi_{1}\left(x_{1}+\lambda a\right) \ldots \varphi_{n}\left(x_{n}+\lambda a\right)\right\rangle \\
=\int d x d y \overline{g\left(y_{1}^{\theta}, \ldots, y_{m}^{\theta}\right)} f\left(x_{1}, \ldots, x_{n}\right)\left\langle\chi_{m}^{\dagger}\left(y_{m}\right) \ldots \chi_{1}^{\dagger}\left(y_{1}\right)\right\rangle\left\langle\varphi_{1}\left(x_{1}\right) \ldots \varphi_{n}\left(x_{n}\right)\right\rangle
\end{gathered}
$$

for any Schwartz test functions $f\left(x_{1}, \ldots, x_{n}\right)$ and $g\left(y_{1}, \ldots, y_{m}\right)$ supported for $0>x_{1}^{0}>x_{2}^{0}>$ $\ldots>x_{n}^{0}$ and $0>y_{1}^{0}>y_{2}^{0}>\ldots>y_{n}^{0}$, for any local fields $\varphi_{1}, \ldots, \varphi_{n}$ and $\chi_{1}, \ldots, \chi_{m}$, and for any $a \in \mathbb{R}^{d}$ which is parallel to the $x^{0}$ plane ( $a^{0}=0$, called "purely spatial" elsewhere). The latter requirement is somewhat analogous to having the Wightman cluster property (2.4) to be satisfied only for spacelike $a .^{20}$

Note that the Minkowski operators can be mapped to Euclidean operators. In particular any $\mathrm{SO}(1, d-1)$ irrep can be mapped to an $\mathrm{SO}(d)$ irrep. This map of irreps originates from the map between the two Lie algebras which have the same complexification. It can then be shown that a pair of conjugate $\mathrm{SO}(1, d-1)$ irreps is mapped to a pair of $\mathrm{SO}(d)$ irreps which are conjugate-reflected to each other. This gives another rationale for the appearance of reflected irreps in the OS axioms.

Remark 2.4. The stated version of OS axioms includes the assumption of real analyticity (2.14) and the bound (2.15). These assumptions are natural from physics perspective; they also easily follow from Wightman axioms. The original OS axioms did not include (2.14) nor (2.15), but included instead a differently stated assumption:

$$
\text { correlators are distributions on }{ }^{0} \mathcal{S},
$$

where ${ }^{0} \mathcal{S}$ is the space of Schwartz test function vanishing at coincident points with all their derivatives.

We would like to discuss here the relation between $(2.14)+(2.15)$ and (2.25). In one direction this is easy: clearly (2.15) implies (2.25). In the other direction it can be shown that (2.25) and other OS axioms (in particular OS positivity and rotation invariance) imply real analyticity (2.14). This is a result of $[2,3]$ and [46]. It is also possible to derive (2.15) from (2.25) and other OS axioms [3]. These issues will be reviewed further in section 9 .

Remark 2.5. In ref. [3], Osterwalder and Schrader introduced an extra assumption on Euclidean correlators, the linear growth condition. We will come back to this in section 9 . Here we would like to stress that this condition is not needed for the implications discussed in the previous paragraph. On the other hand, the linear growth condition was used in ref. [3] when showing that the Euclidean correlators can be Wick-rotated to Lorentzian signature, resulting in Wightman distributions.

\footnotetext{
${ }^{20}$ This is axiom E4 in [2]. Ref. [2] also mentions a stronger axiom E4', but we will be content here with checking the easier axiom E4.
} 


\subsection{Euclidean CFT axioms}

Wightman and OS axioms stated in the previous two sections are standard. We took care to present them for general operator representations and in general $d$. We will now present axioms for Euclidean unitary CFT. Just as OS axioms, these concern correlators in Euclidean signature, but there is an extra assumption of conformal invariance. Another feature of the CFT axioms is that assumptions are imposed on simple building blocks (2- and 3-point functions) from which more complicated correlators can be constructed. Properties of these complicated correlators then follow. The point of our paper is how one can recover OS axioms and (after Wick rotation) Wightman axioms in this setup.

A Euclidean unitary CFT in $\mathbb{R}^{d}(d \geqslant 2)$ deals with local primary operators $\mathcal{O}_{i}(x)$ and with their $n$-point correlation functions $\left\langle\mathcal{O}_{i_{1}}\left(x_{1}\right) \ldots \mathcal{O}_{i_{n}}\left(x_{n}\right)\right\rangle$. Correlators are realanalytic functions defined away from coincident points, which are permutation-invariant as in (2.16). Each primary is characterized by its scaling dimension $\Delta_{i}$ and is an $\mathrm{SO}(d)$ tensor transforming in an irreducible representation $\rho_{i} \cdot{ }^{21}$ The scaling dimensions are real and nonnegative, with the unit operator having dimension zero. The set of scaling dimensions ("spectrum") is assumed to be discrete, by which we mean that there are finitely many $\Delta_{i}$ 's in any finite interval $[a, b] \subset \mathbb{R}^{22}$

The set of all local operators of a CFT consists of primaries $\mathcal{O}_{i}(x)$ and their space-time derivatives $\partial_{\mu_{1}} \ldots \partial_{\mu_{n}} \mathcal{O}_{i}(x)$, often referred to as descendants. The correlation functions of the descendant operators are simply the derivatives of the correlation functions of primary operators. They are well-defined since the correlators of primaries are assumed to be real-analytic.

Parameters $\Delta_{i}$ and $\rho_{i}$ determine transformation properties of $\mathcal{O}_{i}(x)$ under the conformal group $\mathrm{SO}(d+1,1)$, and correlators remain invariant under these standard transformations which we will not write down. These constraints determine the functional form of 1,2,3-point functions. In particular, the unit operator is the only one with a nonzero 1-point function. See, e.g., [20] for a review of these facts.

An important fact that follows from the conformal invariance of correlation functions is that one is allowed to insert an operator at spatial infinity. The primary operator at spatial infinity is defined as

$$
\left\langle\mathcal{O}_{i}(\infty) \cdots\right\rangle \equiv \lim _{L \rightarrow+\infty} L^{2 \Delta_{i}}\left\langle\mathcal{O}_{i}\left(L \widehat{e}_{0}\right) \cdots\right\rangle
$$

To see that this limit exists one can use a conformal map that takes $\infty$ to a finite point and moves no other operators to infinity. After applying this map the limit (2.26) turns into a limit in which all points approach finite values. We conclude that (2.26) exists, and is then of course independent of the concrete conformal map that we chose. In the definition (2.26) we have chosen a particular direction $\left(\widehat{e}_{0}\right)$ for the limit. Using conformal symmetry it is

\footnotetext{
${ }^{21}$ Operators can also be grouped into multiplets of the global symmetry group $G$ which a CFT might have, but we will not discuss global symmetry here. For simplicity we will only consider bosonic operators. More generally one should allow fermionic operators and spinor representations.

${ }^{22}$ There exist $2 \mathrm{~d}$ unitary CFTs, such as the Liouville theory, with a continuous spectrum of scaling dimensions. In this case axioms need to be modified. All known unitary CFTs in $d \geqslant 3$ have a discrete spectrum.
} 
easy to show that (2.26) is independent of this direction, up to a rotation on the indices of $\mathcal{O}_{i}$. In what follows we will always allow for Euclidean CFT correlators to have one of the operators to be at $\infty$.

A Euclidean unitary CFT comes equipped with a conjugation operation $\dagger$, an involutive antilinear operator on the vector space of local operators (including both primaries and descendants), similarly to the OS axioms. Every primary $\mathcal{O}_{i}$ is mapped by $\dagger$ to a conjugate primary $\mathcal{O}_{i}^{\dagger}$ such that the 2-point function $\left\langle\mathcal{O}_{i}^{\dagger} \mathcal{O}_{i}\right\rangle$ does not vanish. The $\mathcal{O}_{i}$ and $\mathcal{O}_{i}^{\dagger}$ have equal scaling dimensions, and transform in the conjugate-reflected irreps. Recall that in section 2.2 we saw that most $\mathrm{SO}(d)$ irreps are self-conjugate-reflected, $\rho_{i} \simeq \bar{\rho}_{i}^{R}$, the only exception being (anti-)chiral tensors in $d=0 \bmod 4$ which are exchanged by this operation. For operators in self-conjugate-reflected irreps we may choose operator basis such that eq. (2.20) holds, which we copy here:

$$
\left(\mathcal{O}_{i}^{\dagger}\right)^{(\mu)}=\Theta_{(\nu)}^{(\mu)} \mathcal{O}_{i}^{(\nu)}
$$

The functional form of the $\left\langle\mathcal{O}_{i}^{\dagger}(x) \mathcal{O}_{i}(y)\right\rangle$ 2-point function is fixed by conformal symmetry:

$$
\left\langle\left(\mathcal{O}_{i}^{\dagger}\right)^{(\mu)}(x) \mathcal{O}_{i}^{(\nu)}(y)\right\rangle=\mathcal{N}_{i} I^{(\mu),(\nu)}(x-y),
$$

where $(\mu),(\nu)$ are collections of tensor indices (of equal length), $I^{(\mu),(\nu)}(x-y)$ is a tensor function depending only on $\Delta_{i}, \rho_{i}$, and $\mathcal{N}_{i}$ is a constant.

We are free to rescale the basis of primaries, multiplying them by some positive constants which should be the same for both $\mathcal{O}_{i}$ and $\mathcal{O}_{i}^{\dagger}$. This transformation clearly preserves the above conditions on $\dagger$, as well as further conditions which will be discussed below, notably (2.32). Using this freedom we can rescale $\mathcal{N}_{i}$ by a positive real number and fix $\left|\mathcal{N}_{i}\right|$ in some arbitrary unimportant way, e.g. so that some component of the 2-point function is of absolute value one at unit separation. On the other hand the phase of $\mathcal{N}_{i}$ cannot be changed in this way. Instead, it is uniquely determined by the positivity condition discussed below.

Positivity is imposed in Euclidean CFT axioms only on 2-point functions. We write it again using the language of states. Basic ket states are $\left|\partial^{(\beta)} \mathcal{O}_{i}^{(\nu)}\right\rangle$ where $\mathcal{O}_{i}^{(\nu)}$ is a primary component and $\partial^{(\beta)}$ an arbitrary derivative. The inner product is defined as

$$
\left\langle\partial^{(\alpha)} \mathcal{O}_{i}^{(\mu)} \mid \partial^{(\beta)} \mathcal{O}_{i}^{(\nu)}\right\rangle=\Theta_{\left(\alpha^{\prime}\right)}^{(\alpha)}\left\langle\partial^{\left(\alpha^{\prime}\right)}\left(\mathcal{O}_{i}^{\dagger}\right)^{(\mu)}\left(x_{N}\right) \partial^{(\beta)} \mathcal{O}_{i}^{(\nu)}\left(x_{S}\right)\right\rangle
$$

i.e. as the value of the shown 2-point function inserting the operators at $x_{S}=(-1,0, \ldots, 0)$ and $x_{N}=(1,0, \ldots, 0)=\left(x_{S}\right)^{\theta} \quad$ (where N,S stands for north, south). For ket states with $i \neq j$ the inner product vanishes since the 2-point function is zero. This inner product is extended by (anti)linearity to the vector space $\mathcal{H}_{0}^{\mathrm{CFT}}$ of finite complex linear combinations of basic ket states. In this language, Euclidean CFT positivity reads exactly as the Wightman and OS positivity: $\langle\Psi \mid \Psi\rangle \geqslant 0$ for all states of this restricted form. More prosaically, this can also be stated that the infinite matrices $M_{i}^{(\alpha)(\mu),(\beta)(\nu)}$ built out of 2point functions in the r.h.s. of (2.29) are all positive semidefinite when restricted to finite subspaces. 
CFT positivity can be analyzed primary by primary, and it depends only on the primary 2-point function, eq. (2.28) which determines the full matrix $M_{i}^{(\alpha)(\mu),(\beta)(\nu)}$. Clearly, only one phase of the normalization constant $\mathcal{N}_{i}$ in eq. (2.28) can give rise to a positive definite matrix, so that phase is uniquely fixed. Once the phase of $\mathcal{N}_{i}$ is fixed, positivity for a given primary depends only on its $\Delta, \rho$. It then can be shown that CFT positivity holds if and only if every $\Delta$, in addition to being real and non-negative, lies above a certain minimal $\rho$-dependent value ("unitarity bound"):

$$
\Delta \geqslant \Delta_{\min }(\rho)
$$

These unitarity bounds are documented in the literature, e.g. we have $\Delta_{\min }=d / 2-1$ for scalars, and $d+\ell-2$ for spin- $\ell, \ell \geqslant 1$. For arbitrary $\mathrm{SO}(d)$ representations see [47]. ${ }^{23}$

For future uses, we wish to define the CFT Hilbert space $\mathcal{H}^{\mathrm{CFT}}$ via completion of $\mathcal{H}_{0}^{\mathrm{CFT}}$, after modding out by zero norm states (for operators saturating the unitarity bounds, some descendants have zero norm). This can be done abstractly, or explicitly using a basis as we now describe. Throwing out zero-norm descendants, the remaining states can be organized choosing an orthonormal basis. We may choose such a basis independently among descendants of each primary, and then combine all these bases, e.g. in the order of nondecreasing scaling dimensions. The elements of $\mathcal{H}^{\mathrm{CFT}}$ are then formal linear combinations $\sum_{n} c_{n}|n\rangle$, where $|n\rangle$ are orthonormal basis elements, and $c_{n}$ is an arbitrary complex $\ell_{2}$ sequence. The norm on $\mathcal{H}^{\mathrm{CFT}}$ is the $\ell_{2}$ norm of the sequence $c_{n}$. Restricting to sequences $c_{n}$ which have only a finite number of nonzero elements, we get elements of $\mathcal{H}_{0}^{\text {CFT }}$ (modulo the zero-norm states).

Let us continue with the axioms. CFT hermiticity condition is imposed only on the 2-point and 3-point functions, namely:

$$
\left\langle\left(\mathcal{O}_{i}^{\dagger}\right)^{(\mu)}\left(x_{1}\right) \mathcal{O}_{i}^{(\nu)}\left(x_{2}\right)\right\rangle=\overline{\left\langle\mathcal{O}_{i}^{(\mu)}\left(x_{1}^{\theta}\right)\left(\mathcal{O}_{i}^{\dagger}\right)^{(\nu)}\left(x_{2}^{\theta}\right)\right\rangle},
$$

which is also a consequence CFT positivity and in particular fixed the phase of $\mathcal{N}_{i}$ up to a sign, ${ }^{24}$ and

$$
\left\langle\mathcal{O}_{i}^{(\mu)}\left(x_{1}\right) \mathcal{O}_{j}^{(\nu)}\left(x_{2}\right) \mathcal{O}_{k}^{(\lambda)}\left(x_{3}\right)\right\rangle=\overline{\left\langle\left(\mathcal{O}_{i}^{\dagger}\right)^{(\mu)}\left(x_{1}^{\theta}\right)\left(\mathcal{O}_{j}^{\dagger}\right)^{(\nu)}\left(x_{2}^{\theta}\right)\left(\mathcal{O}_{k}^{\dagger}\right)^{(\lambda)}\left(x_{3}^{\theta}\right)\right\rangle} .
$$

\footnotetext{
${ }^{23}$ We chose to express CFT positivity inserting operators at the points $( \pm 1,0, \ldots, 0)$ which corresponds to the N-S quantization (see [48]) and will facilitate the comparison with the Osterwalder-Schrader reflection positivity. Equivalently, one could go via a conformal transformation to the more familiar radial quantization corresponding to inserting the operators at 0 and $\infty$. CFT positivity is then equivalent to radial quantization states having positive norm on every level, which is how the unitarity bounds are usually worked out in Euclidean CFTs [47]. In mathematical language, this latter condition corresponds to having a positive-definite Shapovalov form on the parabolic Verma module. Recent work [49, 50] explained how the determinant formulas by Jantzen [51] provide a rigorous justification of the Euclidean unitarity bounds (both in the necessary and sufficient directions).

${ }^{24}$ For the special case $x_{1}=x_{N}, x_{2}=x_{S}$, eq. (2.31) is nothing but hermiticity of the matrix $M_{i}^{(\mu),(\nu)}$, a consequence of positive-semidefiniteness. The general case reduces to the special one mapping $x_{1}, x_{2}$, to $x_{N}, x_{S}$ by a conformal transformation (both sides of (2.31) have the same conformal transformation properties).
} 
for any 3 primaries $\mathcal{O}_{i}, \mathcal{O}_{j}, \mathcal{O}_{k}{ }^{25}$ Similarly to the 2-point function case, this condition can be simplified using the conformally invariant tensor structures, with an important difference that the normalization of operators has already been fixed. Conformal invariance constrains the 3 -point functions to take the form

$$
\left\langle\mathcal{O}_{i}^{(\mu)}\left(x_{1}\right) \mathcal{O}_{j}^{(\nu)}\left(x_{2}\right) \mathcal{O}_{k}^{(\lambda)}\left(x_{3}\right)\right\rangle=\sum_{a=1}^{N_{i j k}} f_{i j k}^{a}\left\langle\mathcal{O}_{i}^{(\mu)}\left(x_{1}\right) \mathcal{O}_{j}^{(\nu)}\left(x_{2}\right) \mathcal{O}_{k}^{(\lambda)}\left(x_{3}\right)\right\rangle_{a},
$$

where $\left\langle\mathcal{O}_{i}^{(\mu)}\left(x_{1}\right) \mathcal{O}_{j}^{(\nu)}\left(x_{2}\right) \mathcal{O}_{k}^{(\lambda)}\left(x_{3}\right)\right\rangle_{a}$ span the finite-dimensional space (of dimension $N_{i j k}$ ) of solutions of conformal invariance constraints on the 3-point functions of the operators with given $\Delta_{s}, \rho_{s}(s=i, j, k)$. On the other hand the coefficients $f_{i j k}^{a} \in \mathbb{C}$ are not fixed by conformal symmetry (no sum on $i, j, k$ in the r.h.s. of (2.33)). We often refer to $f_{i j k}^{a}$ as the "OPE coefficients." It is always possible to choose the basis structures $\left\langle\mathcal{O}_{i}^{(\mu)}\left(x_{1}\right) \mathcal{O}_{j}^{(\nu)}\left(x_{2}\right) \mathcal{O}_{k}^{(\lambda)}\left(x_{3}\right)\right\rangle_{a}$ to satisfy the hermiticity constraint (2.32) individually, in which case the OPE coefficients must satisfy

$$
\left(f_{i j k}^{a}\right)^{*}=f \frac{a}{i j k},
$$

where the barred indices refer to the conjugate operators $\mathcal{O}_{i}^{\dagger}, \mathcal{O}_{j}^{\dagger}, \mathcal{O}_{k}^{\dagger}$. In particular, when all three operators are self-conjugate-reflected i.e. satisfy (2.27), the OPE coefficients $f_{i j k}^{a}$ must be real.

Finally, unitary CFTs enjoy a convergent operator product expansion (OPE). This means that any correlation function ${ }^{26}$ satisfies

$$
\begin{aligned}
&\left\langle\mathcal{O}_{i}^{(\mu)}\left(x_{1}\right) \mathcal{O}_{j}^{(\nu)}\left(x_{2}\right) \mathcal{O}_{m}^{(\rho)}\left(x_{3}\right) \cdots\right\rangle \\
&=\sum_{k} \sum_{a=1}^{N_{i j k}} f_{i j k}^{a} C_{a,(\lambda)}^{(\mu)(\nu)}\left(x_{1}, x_{2}, x_{0}, \partial_{0}\right)\left\langle\left(\mathcal{O}_{k}^{\dagger}\right)^{(\lambda)}\left(x_{0}\right) \mathcal{O}_{m}^{(\rho)}\left(x_{3}\right) \cdots\right\rangle,
\end{aligned}
$$

where the first sum runs over all primary operators $\mathcal{O}_{k}$ in the theory, and $C_{a,(\lambda)}^{(\mu)(\nu)}\left(x_{1}, x_{2}, x_{0}, \partial_{0}\right)$ is a formal sum of the form

$$
C_{a,(\lambda)}^{(\mu)(\nu)}\left(x_{1}, x_{2}, x_{0}, \partial_{0}\right)=\sum_{\alpha} C_{a,(\lambda), \alpha}^{(\mu)(\nu)}\left(x_{1}, x_{2}, x_{0}\right)\left(\partial / \partial x_{0}\right)^{\alpha} .
$$

This differential operator is determined by conformal symmetry ${ }^{27}$ and depends only on $\Delta_{s}, \rho_{s}(s=i, j, k)$. Here $(\partial / \partial x)^{\alpha}=\left(\partial / \partial x^{0}\right)^{\alpha_{0}} \ldots\left(\partial / \partial x^{d-1}\right)^{\alpha_{d-1}}$ with $\alpha=\left(\alpha_{0}, \ldots, \alpha_{d-1}\right) \in$ $\left(\mathbb{Z}_{\geqslant 0}\right)^{d}$ a multiindex. Convergence of OPE means that the sum (2.35), with $C$ expanded as in (2.36), converges whenever

$$
x_{1}, x_{2} \in B\left(x_{0}, R\right), \quad R=\min \left(\left|x_{3}-x_{0}\right|,\left|x_{4}-x_{0}\right|, \ldots\right)
$$

\footnotetext{
${ }^{25}$ Note that since $\dagger$ is involutive, this covers the case when an operator and its conjugate are interchanged between the two sides of this equation.

${ }^{26}$ Importantly, we allow here for one of the operators to be inserted at spatial infinity.

${ }^{27}$ There is an ambiguity when $\mathcal{O}_{k}$ is in a short conformal representation (in unitary theories this happens only if $\mathcal{O}_{k}$ is a conserved current or a free field). This subtlety will not play any role in this paper.
} 
where $B\left(x_{0}, R\right)$ is an open ball centered at $x_{0}$ and of radius $R$. In other words, OPE converges whenever $x_{1}$ and $x_{2}$ are the two closest operator insertions to $x_{0}$ (in Euclidean distance). Convergence should be understood carefully as follows. For each $\mathcal{O}_{k}^{\dagger}$ in the r.h.s. of (2.35), and for each $n \in \mathbb{Z}_{+}$, we perform finite summation over $a, \lambda$, and all multiindices $\alpha$ with $|\alpha|=n$. We are left then with the doubly infinite sum

$$
\sum_{k} \sum_{n=0}^{\infty} g_{k, n}\left(\left\{x_{i}\right\}\right) .
$$

This doubly infinite sum has to converge absolutely for every $x_{1}, x_{2}$ as in (2.37). ${ }^{28}$

That the same coefficients $f_{i j k}^{a}$ appear in the OPE (2.35) and the 3-point function (2.33) follows immediately by using the OPE inside the latter 3-point function.

Local Euclidean CFTs contain the conserved stress tensor operator $T_{\mu \nu}$ of dimension $d$, and in case of continuous global symmetry, conserved global symmetry currents $J_{\mu}$ of dimensions $d-1$. We will not discuss here additional axioms involving 3-point functions and OPE coefficients of these operators, related to their conservation and Ward identities, see e.g. $[52,53]$.

Remark 2.6. The just given Euclidean CFT axioms are more careful in what concerns reality constraints than the set of CFT rules gathered in the conformal bootstrap review [20]. They are also more economical: e.g. Ref. [20] assumed OS reflection positivity and clustering for $n$-point functions, which for us will be theorems to prove, not assumptions. Ref. [20] also included some constraints on the CFT data which emerge when considering CFT in Minkowski signature, most notably the Averaged Null Energy Condition (ANEC). In this paper we will establish all Wightman axioms for scalar Minkowski CFT 4-point functions from the Euclidean CFT axioms, but we will not discuss ANEC. A proof of ANEC [54] has been given using the Haag-Kastler axioms for general QFT. CFT arguments have also been given in $[45,55]$, but they rely on some assumptions which have not been rigorously proven from axioms. It would be interesting to fill these gaps and establish ANEC as a theorem from Euclidean CFT axioms. ${ }^{29}$

\section{$3 \quad$ Euclidean CFT $\Rightarrow$ Osterwalder-Schrader}

In this section we will discuss some simple consequences of CFT axioms and in particular will show that they imply OS axioms for 4-point functions (the case of higher-point functions is more subtle and is discussed in appendix B). Here we will prove only the OS reflection-positivity and the cluster property. The "Euclidean temperedness" bound (2.15)

\footnotetext{
${ }^{28}$ The requirement of absolute convergence can be somewhat relaxed, see section 3.1.

29 The argument in [45] uses an OPE asymptotic expansion on the second sheet, outside of the range of convergence of the OPE rigorously implied by the Euclidean CFT axioms (see appendix A.1 where we review this method going back to [4]). In [55] ANEC is derived using manipulations with a generalization of the Lorentzian inversion formula of [56], of which some have not been rigorously justified. For example, the derivation starts with the Euclidean inversion formula, which is readily justified from Harmonic analysis only for external scaling dimensions on principal series and square-integrable correlators, none of which is generically the case in the required setup.
} 
will follow from our arguments in the following sections, where we establish power-law bounds on CFT correlation functions. The remaining OS axioms are a subset of the CFT axioms.

\subsection{OS reflection positivity}

In this section we prove OS positivity for compactly-supported test functions. The extension to Schwartz functions is easy once we establish (2.15) for CFT correlators, see remark 4.2.

To someone familiar with OPE in CFTs, the goal of this section may seem straightforward to achieve: in usual CFT treatments, one can express any state as a sum of "single operator states", created by action of a single insertion of primaries and their descendants on the vacuum, and our two-point positivity axiom makes sure that the inner products of such states are positive-semidefinite. The part of this wisdom that doesn't work immediately in our setup is being able to express states in the OS Hilbert space as infinite OPE sums. Our OPE axiom is much weaker than this statement, in particular the truncation order for the OPE needed to achieve a given precision $\epsilon$ in (2.35) may a priori depend on everything in the left-hand side, including coordinates $x_{i}$ and the "spectator" operators $\mathcal{O}_{m}, \ldots$, in an arbitrary fashion. (On the contrary, for a Hilbert space OPE statement, the truncation order is good for all choices of spectator operators as long as an appropriate norm remains bounded by a fixed constant.) In spite of this difficulty, in this and the next section we will be able to recover reflection positivity for $n$-point functions up to $n \leq 4$ and Hilbert space OPE convergence for states created by up to 2 operator insertions, but doing so requires some care.

First let us slightly reformulate the OPE convergence property. Consider an $n$-point correlation function with operators inserted at $x_{1} \ldots x_{n}$. Let $S$ be a hyperplane and $x_{0}$ be a point such that $x_{1}, x_{2}, x_{0}$ are on one side of $S$ while all the other points $x_{i}, i>2$, are on the other side. Using a conformal transformation we can map $S$ to a sphere $S^{\prime}$ so that $x_{0}$ is mapped to the center of $S^{\prime}$ which we denote by $x_{0}^{\prime}$. Let $x_{i}^{\prime}$ denote the positions of all the other points $x_{i}$ after this map. We can then use the OPE (2.35) for the correlation function evaluated at $x_{i}^{\prime}$,

$$
\begin{aligned}
\left\langle\mathcal{O}_{i}^{(\mu)}\left(x_{1}^{\prime}\right) \mathcal{O}_{j}^{(\nu)}\right. & \left.\left(x_{2}^{\prime}\right) \mathcal{O}_{m}^{(\rho)}\left(x_{3}^{\prime}\right) \cdots\right\rangle \\
& =\sum_{k} \sum_{a=1}^{N_{i j k}} f_{i j k}^{a} C_{a,(\lambda)}^{(\mu)(\nu)}\left(x_{1}^{\prime}, x_{2}^{\prime}, x_{0}^{\prime}, \partial_{0^{\prime}}\right)\left\langle\left(\mathcal{O}_{k}^{\dagger}\right)^{(\lambda)}\left(x_{0}^{\prime}\right) \mathcal{O}_{m}^{(\rho)}\left(x_{3}^{\prime}\right) \cdots\right\rangle .
\end{aligned}
$$

Transforming this expansion term-by term to the original coordinates $x_{i}$ we find the convergent expansion (with convergence understood in the same sense as in the previous section $\left.^{30}\right)$

$$
\begin{aligned}
\left\langle\mathcal{O}_{i}^{(\mu)}\left(x_{1}\right) \mathcal{O}_{j}^{(\nu)}\right. & \left.\left(x_{2}\right) \mathcal{O}_{m}^{(\rho)}\left(x_{3}\right) \cdots\right\rangle \\
& =\sum_{k} \sum_{a=1}^{N_{i j k}} f_{i j k}^{a} \mathfrak{C}_{a,(\lambda)}^{(\mu)(\nu)}\left(x_{1}, x_{2}, x_{0}, \mathcal{D}_{0}\right)\left\langle\left(\mathcal{O}_{k}^{\dagger}\right)^{(\lambda)}\left(x_{0}\right) \mathcal{O}_{m}^{(\rho)}\left(x_{3}\right) \cdots\right\rangle .
\end{aligned}
$$

\footnotetext{
${ }^{30}$ Careful reading of the argument below shows that, in the 4-point case, the requirement of absolute convergence of (2.38) could be replaced by a weaker requirement that we can find any subsequence of partial sums of (2.38) which approximates the correlator pointwise.
} 
Here the differential operators $\mathcal{D}^{(\alpha)}$ are simply the derivatives $\left(\frac{\partial}{\partial x^{\prime}}\right)^{(\alpha)}$ expressed in the original coordinates $x$, and conjugated by the conformal transformation factor of $\mathcal{O}_{k}^{\dagger}$. The functions $\mathfrak{C}_{a,(\lambda)}^{(\mu)(\nu)}$ are obtained from $C_{a,(\lambda)}^{(\mu)(\nu)}$ by our conformal transformation. The important point is that truncation of $C_{a,(\lambda)}^{(\mu)(\nu)}$ in order of derivatives $\partial^{(\alpha)}$ corresponds to truncation of $\mathfrak{C}_{a,(\lambda)}^{(\mu)(\nu)}$ in order of operators $\mathcal{D}^{(\alpha)}$.

We now specialize to $S$ being the $x^{0}=0$ plane, $x_{0}=x_{S}=(-1,0, \ldots)$, and take $S^{\prime}$ to be the unit sphere with the center $x_{0}^{\prime}=0$. Then the derivatives $\left(\frac{\partial}{\partial x^{\prime}}\right)^{(\alpha)} \mathcal{O}_{k}^{(\mu)}\left(x_{0}^{\prime}\right)$ are eigenstates of the standard dilatation generator $D$ with eigenvalues $\Delta_{k}+|\alpha|$. Note that $D$ has two fixed points: $x_{0}^{\prime}=0$ and infinity. Applying our conformal map, we find that the derivatives $\mathcal{D}^{(\alpha)} \mathcal{O}_{k}^{(\mu)}\left(x_{S}\right)$ are in turn eigenstates, with the same eigenvalues, of the conformal generator $D^{\prime}=\left(K^{0}-P^{0}\right) / 2$ that preserves $x_{S}$ and $x_{N}=x_{S}^{\theta}$ (which is the image of infinity under our conformal map) and acts by dilatations near these two points. This, together with the conformal invariance and diagonality of 2-point functions, implies

$$
\left\langle\left(\mathcal{D}^{(\alpha)}\right)^{\theta} \mathcal{O}_{j}^{\dagger(\mu)}\left(x_{N}\right) \mathcal{D}^{(\beta)} \mathcal{O}_{k}^{(\nu)}\left(x_{S}\right)\right\rangle \propto \delta_{|\alpha|,|\beta|} \delta_{j, k},
$$

where $\left(\mathcal{D}^{(\alpha)}\right)^{\theta}$ is obtained from $\mathcal{D}^{(\alpha)}$ by replacing $x \rightarrow x^{\theta}$.

The OPE (3.2) gives an expansion for ket states $|\Psi\rangle \in \mathcal{H}_{0}^{\mathrm{OS}}$ created by two local operators in terms of ket states created by a single operator, which are elements of $\mathcal{H}_{0}^{\mathrm{CFT}}$. We would like to have a dual version of this expansion for bra states $\langle\Psi|$. For this we need to understand how the OPE transforms under the conjugation. Note that the formal differential operators $\mathfrak{C}_{a,(\lambda)}^{(\mu)(\nu)}\left(x_{1}, x_{2}, x_{0}, \mathcal{D}_{0}\right)$ can be uniquely determined by the equation

$$
\left\langle\mathcal{O}_{1}^{(\mu)}\left(x_{1}\right) \mathcal{O}_{2}^{(\nu)}\left(x_{2}\right) \mathcal{O}_{3}^{(\rho)}\left(x_{3}\right)\right\rangle_{a}=\mathfrak{C}_{a,(\lambda)}^{(\mu)(\nu)}\left(x_{1}, x_{2}, x_{0}, \mathcal{D}_{0}\right)\left\langle\left(\mathcal{O}_{3}^{\dagger}\right)^{(\lambda)}\left(x_{0}\right) \mathcal{O}_{3}^{(\rho)}\left(x_{3}\right)\right\rangle
$$

where it is understood that the points are arranged as above, so that the formal sum defined by $\mathfrak{C}_{a,(\lambda)}^{(\mu)(\nu)}\left(x_{1}, x_{2}, x_{0}, \mathcal{D}_{0}\right)$ actually converges. By applying complex conjugation on both sides and using the 2-point and 3-point hermiticity constraints (2.31) and (2.32) we find

$$
\left\langle\left(\mathcal{O}_{1}^{\dagger}\right)^{(\mu)}\left(x_{1}^{\theta}\right)\left(\mathcal{O}_{2}^{\dagger}\right)^{(\nu)}\left(x_{2}^{\theta}\right)\left(\mathcal{O}_{3}^{\dagger}\right)^{(\rho)}\left(x_{3}^{\theta}\right)\right\rangle_{a}=\left[\mathfrak{C}_{a,(\lambda)}^{(\mu)(\nu)}\left(x_{1}, x_{2}, x_{0}, \mathcal{D}_{0}\right)\right]^{*}\left\langle\mathcal{O}_{3}^{(\lambda)}\left(x_{0}^{\theta}\right)\left(\mathcal{O}_{3}^{\dagger}\right)^{(\rho)}\left(x_{3}^{\theta}\right)\right\rangle,
$$

which implies that

$$
\left[\mathfrak{C}_{a,(\lambda)}^{(\mu)(\nu)}\left(x_{1}^{\theta}, x_{2}^{\theta}, x_{0}^{\theta}, \mathcal{D}_{0}^{\theta}\right)\right]^{*}=\widetilde{\mathfrak{C}}_{a,(\lambda)}^{(\mu)(\nu)}\left(x_{1}, x_{2}, x_{0}, \mathcal{D}_{0}\right),
$$

where $\widetilde{\mathfrak{C}}_{a,(\lambda)}^{(\mu)(\nu)}\left(x_{1}, x_{2}, x_{0}, \mathcal{D}_{0}\right)$ is the formal sum that appears in the OPE for operators with conjugate-reflected quantum numbers.

We are now ready to prove OS positivity for 4-point functions. Let $\left|\Psi_{0}\right\rangle$ be an OS ket state involving at most two local operators, i.e. ${ }^{31}$

$$
\left|\Psi_{0}\right\rangle=\sum_{i, j, \alpha, \beta} \int d x_{1} d x_{2} f_{i, j,(\alpha)(\beta)}\left(x_{1}, x_{2}\right)\left|\mathcal{O}_{i}^{(\alpha)}\left(x_{1}\right) \mathcal{O}_{j}^{(\beta)}\left(x_{2}\right)\right\rangle
$$

\footnotetext{
${ }^{31}$ In the notation of section 2.2 this could be written as $\sum_{i, k, \alpha, \beta}\left|\psi\left(f_{i, j,(\alpha),(\beta)}, \mathcal{O}_{i}^{(\alpha)}, \mathcal{O}_{j}^{(\beta)}\right)\right\rangle$.
} 
where $f\left(x_{1}, x_{2}\right)$ is a compactly supported test function vanishing unless $0>x_{1}^{0}>x_{2}^{0}$. (Terms with one or no operators are realized by setting one or both operators to the identity.) Since by Euclidean CFT axioms the correlation functions are real-analytic, the integrals that appear in the expression for $\left\langle\Psi_{0} \mid \Psi_{0}\right\rangle$ can be approximated by finite Riemann sums, reflection-symmetric if necessary. This implies that, for any $\varepsilon>0$, we can pass from $\left|\Psi_{0}\right\rangle$ to a ket state $|\Psi\rangle$ which is created by a finite linear combination of insertions of up to two local operators with $x_{1}^{0}, x_{2}^{0}<0$ :

$$
|\Psi\rangle=\sum_{i, j, \alpha, \beta, x_{1}, x_{2}} c_{i, j,(\alpha)(\beta), x_{1}, x_{2}}\left|\mathcal{O}_{i}^{(\alpha)}\left(x_{1}\right) \mathcal{O}_{j}^{(\beta)}\left(x_{2}\right)\right\rangle,
$$

and has the property that

$$
\left|\left\langle\Psi_{0} \mid \Psi_{0}\right\rangle-\left\langle\Psi_{0} \mid \Psi\right\rangle\right|<\varepsilon, \quad\left|\left\langle\Psi_{0} \mid \Psi\right\rangle-\langle\Psi \mid \Psi\rangle\right|<\varepsilon,
$$

so that as a result

$$
\left|\left\langle\Psi_{0} \mid \Psi_{0}\right\rangle-\langle\Psi \mid \Psi\rangle\right|<2 \varepsilon
$$

We are therefore reduced to proving the nonnegativity of $\langle\Psi \mid \Psi\rangle$.

Now, the OPE convergence axiom implies that, for any $\varepsilon>0$, starting from $|\Psi\rangle$ and using the OPE (3.2) in the half-space $x^{0}<0$, we can construct a state $|\psi\rangle=\left|\psi_{\Lambda}\right\rangle \in \mathcal{H}_{0}^{\mathrm{CFT}}$ created by a finite linear combination of local operators at $x_{S}$ such that

$$
|\langle\Psi \mid \psi\rangle-\langle\Psi \mid \Psi\rangle|<\varepsilon
$$

Here $\Lambda$ is an OPE truncation cutoff which we need to increase appropriately as $\varepsilon$ gets smaller. Namely, we will obtain $|\psi\rangle$ by keeping in the OPE all terms with $\Delta_{k}+|\alpha|<\Lambda$, where $\Delta_{k}$ is the dimension of a primary $\mathcal{O}_{k}$ appearing in the OPE, and $\alpha$ is the order of the descendant $\mathcal{D}^{(\alpha)} \mathcal{O}_{k}$.

We can then repeat this procedure in the upper half-plane $x^{0}>0$ and construct a state $\left\langle\psi^{\prime}\right|=\left\langle\psi_{\Lambda^{\prime}}\right|$ of local operators inserted at $x_{N}$ such that

$$
\left|\langle\Psi \mid \psi\rangle-\left\langle\psi^{\prime} \mid \psi\right\rangle\right|<\varepsilon
$$

Eq. (3.6) and the reality constraint (2.34) for the OPE coefficients imply that the state $\left\langle\psi^{\prime}\right|$ differs from $\langle\psi|$ at most by where the OPE expansion was truncated. Furthermore, we can always assume that $\left\langle\psi^{\prime}\right|$ contains at least all the terms that $\langle\psi|$ does (i.e. $\Lambda^{\prime} \geqslant \Lambda$ ), since adding more OPE terms to $\left\langle\psi^{\prime}\right|$ can only improve (3.12).

Eq. (3.3) then implies that $\left\langle\psi^{\prime} \mid \psi\right\rangle=\langle\psi \mid \psi\rangle$ and therefore

$$
|\langle\Psi \mid \psi\rangle-\langle\psi \mid \psi\rangle|<\varepsilon
$$

Combining this with (3.11) we conclude:

$$
|\langle\Psi \mid \Psi\rangle-\langle\psi \mid \psi\rangle|<2 \varepsilon
$$

Since by the CFT positivity axiom $\langle\psi \mid \psi\rangle$ is non-negative, we conclude that $\langle\Psi \mid \Psi\rangle$ is also non-negative. This completes the proof of OS positivity for states created by up to two operator insertions. 


\subsection{Denseness and Hilbert space implications}

Here we will describe some useful byproducts of the just given argument. Note that (3.11) and (3.13), in addition to (3.14), also implies

$$
\left\|\Psi-\psi_{\Lambda}\right\| \equiv\left\langle\Psi-\psi_{\Lambda} \mid \Psi-\psi_{\Lambda}\right\rangle=\langle\Psi \mid \Psi\rangle-\left\langle\Psi \mid \psi_{\Lambda}\right\rangle+\left\langle\psi_{\Lambda} \mid \psi_{\Lambda}\right\rangle-\left\langle\psi_{\Lambda} \mid \Psi\right\rangle<2 \varepsilon
$$

This means that any $|\Psi\rangle$ can be approximated arbitrarily well by a $\left|\psi_{\Lambda}\right\rangle \in \mathcal{H}_{0}^{\mathrm{CFT}}$. In other words, $\mathcal{H}_{0}^{\mathrm{CFT}}$ is a dense subspace of the vector space of $\Psi$ 's.

This fact has a simple but quite powerful consequence involving the CFT Hilbert space $\mathcal{H}^{\mathrm{CFT}}$, defined in section 2.3 as the completion of $\mathcal{H}_{0}^{\mathrm{CFT}}$. Eq. (3.15) implies, using the triangle inequality $\left\|\psi_{\Lambda_{1}}-\psi_{\Lambda_{2}}\right\| \leqslant\left\|\Psi-\psi_{\Lambda_{1}}\right\|+\left\|\Psi-\psi_{\Lambda_{2}}\right\|$, that the states $\left|\psi_{\Lambda}\right\rangle$ corresponding to smaller and smaller $\varepsilon$ form a Cauchy sequence. Therefore, these states have a limit in $\mathcal{H}^{\mathrm{CFT}}$ as $\Lambda \rightarrow \infty$, which we call $\left|\psi_{\infty}\right\rangle$. This $\psi_{\infty}$ is nothing but the full, untruncated, OPE expansion of the state $\Psi$. We claim that the map mapping $\Psi$ 's to the corresponding $\psi_{\infty}$ 's is isometric, i.e. it preserves the inner products:

$$
\langle\Phi \mid \Psi\rangle=\left\langle\varphi_{\infty} \mid \psi_{\infty}\right\rangle .
$$

Here the inner product on the l.h.s. is the OS inner product, computed using CFT 4point functions with operators inserted in the lower and upper half-spaces, while the inner product in the r.h.s. is the $\mathcal{H}^{\mathrm{CFT}}$ inner product, defined as the limit of $\mathcal{H}_{0}^{\mathrm{CFT}}$ inner product removing the cutoff:

$$
\left\langle\varphi_{\infty} \mid \psi_{\infty}\right\rangle:=\lim _{\Lambda \rightarrow \infty}\left\langle\varphi_{\Lambda} \mid \psi_{\Lambda}\right\rangle .
$$

The proof of (3.16) is straightforward. We write:

$$
\begin{gathered}
\left\langle\varphi_{\Lambda} \mid \psi_{\Lambda}\right\rangle=\left\langle\Phi+\left(\varphi_{\Lambda}-\Phi\right) \mid \Psi+\left(\psi_{\Lambda}-\Psi\right)\right\rangle=\langle\Phi \mid \Psi\rangle+\operatorname{err}(\Lambda) \\
\operatorname{err}(\Lambda)=\left\langle\varphi_{\Lambda}-\Phi \mid \Psi\right\rangle+\left\langle\Phi \mid \psi_{\Lambda}-\Psi\right\rangle+\left\langle\varphi_{\Lambda}-\Phi \mid \psi_{\Lambda}-\Psi\right\rangle .
\end{gathered}
$$

By eq. (3.15) we know that $\left\|\Psi-\psi_{\Lambda}\right\|,\left\|\Phi-\varphi_{\Lambda}\right\|$ go to zero as $\Lambda \rightarrow \infty$. Hence, $\operatorname{err}(\Lambda) \rightarrow 0$ and (3.16) is proved.

Eqs. (3.16) and (3.17) mean that OPE converges in the sense of the CFT Hilbert space. This property is often used in the CFT literature (see section 6.2). Note that CFT axioms in section 2.3 only assume pointwise OPE convergence, which is a weaker statement. Curiously, by the given arguments, pointwise OPE convergence plus CFT positivity imply Hilbert space convergence, at least for the 4-point functions.

In the above argument we used the Hilbert space $\mathcal{H}^{\mathrm{CFT}}$, the completion of $\mathcal{H}_{0}^{\mathrm{CFT}}$. We may introduce a second Hilbert space as the completion of the space of $\Psi$ 's, call it $\mathcal{H}^{(2)}$. This Hilbert space contains e.g. all $\Psi_{0}$ states (3.7). (Similarly to (3.15), eqs. (3.9) and (3.10) imply that $\Psi$ states are dense in the $\Psi_{0}$ states.) Although $\mathcal{H}^{(2)}$ may look like a "bigger" space than $\mathcal{H}^{\mathrm{CFT}}$, actually it's not. Indeed, the map from $\Psi$ to $\psi_{\infty}$ extends to an isometric map from $\mathcal{H}^{(2)}$ to $\mathcal{H}^{\mathrm{CFT}}$. In other words, eq. (3.16) remains true for any $\Phi, \Psi \in \mathcal{H}^{(2)}$. Since we can view $\mathcal{H}_{0}^{\mathrm{CFT}}$ as a subspace of the space of $\Psi$ 's, we also have that this map is surjective. The Hilbert spaces $\mathcal{H}^{(2)}$ and $\mathcal{H}^{\mathrm{CFT}}$ are thus unitarily equivalent and may be identified. 


\subsection{OS clustering}

Here we will derive the OS clustering (2.24) from CFT axioms. We will consider only $m+n=4$, i.e. when the left-hand side of $(2.24)$ can be written in terms of a 4 -point function (this also covers $m+n<4$ since we can choose some of $\varphi$ 's or $\chi$ 's to be the trivial identity field). We can assume that all $\chi$ 's and $\varphi$ 's in (2.24) are primary fields, since any derivatives can be integrated by parts.

First as a general remark, assuming OS positivity, clustering (for any $m, n$ ) only needs to be established point-wise, i.e.

$$
\begin{aligned}
\lim _{\lambda \rightarrow \infty}\left\langle\chi_{m}^{\dagger}\left(y_{m}\right) \ldots \chi_{1}^{\dagger}\left(y_{1}\right) \varphi_{1}\left(x_{1}+\lambda a\right) \ldots \varphi_{n}\left(x_{n}+\lambda a\right)\right\rangle \\
=\left\langle\chi_{m}^{\dagger}\left(y_{m}\right) \ldots \chi_{1}^{\dagger}\left(y_{1}\right)\right\rangle\left\langle\varphi_{1}\left(x_{1}\right) \ldots \varphi_{n}\left(x_{n}\right)\right\rangle .
\end{aligned}
$$

This follows from the dominated convergence theorem. Indeed, OS positivity and translation invariance (recall that we only consider $a^{0}=0$ !) implies a uniform in $\lambda$ bound $^{32}$

$$
\begin{aligned}
\mid\left\langle\chi_{m}^{\dagger}\left(y_{m}\right)\right. & \left.\ldots \chi_{1}^{\dagger}\left(y_{1}\right) \varphi_{1}\left(x_{1}+\lambda a\right) \ldots \varphi_{n}\left(x_{n}+\lambda a\right)\right\rangle\left.\right|^{2} \\
& \leqslant\left\langle\chi_{m}^{\dagger}\left(y_{m}\right) \ldots \chi_{1}^{\dagger}\left(y_{1}\right) \chi_{1}\left(y_{1}^{\theta}\right) \ldots \chi_{n}\left(y_{n}^{\theta}\right)\right\rangle \times\left\langle\varphi_{n}^{\dagger}\left(x_{n}^{\theta}\right) \ldots \varphi_{1}^{\dagger}\left(x_{1}^{\theta}\right) \varphi_{1}\left(x_{1}\right) \ldots \varphi_{n}\left(x_{n}\right)\right\rangle .
\end{aligned}
$$

It then follows that the integrand in (2.24) is bounded by a $\lambda$-independent integrable function, and the dominated convergence theorem is applicable.

Going back to the 4-point function case which is our focus in this section, let us start with $m=n=2$. Since we already proved OS positivity for states created by at most two operators (section 3.1), in this case we can rely on the above observation and we only need to check the point-wise limit:

$$
\lim _{\lambda \rightarrow \infty}\left\langle\chi_{2}^{\dagger}\left(y_{2}\right) \chi_{1}^{\dagger}\left(y_{1}\right) \varphi_{1}\left(x_{1}+\lambda a\right) \varphi_{2}\left(x_{2}+\lambda a\right)\right\rangle=\left\langle\chi_{2}^{\dagger}\left(y_{2}\right) \chi_{1}^{\dagger}\left(y_{1}\right)\right\rangle\left\langle\varphi_{1}\left(x_{1}\right) \varphi_{2}\left(x_{2}\right)\right\rangle .
$$

To see this, we apply the $\operatorname{OPE}(3.2)$ to $\chi_{2}^{\dagger}\left(y_{2}\right) \chi_{1}^{\dagger}\left(y_{1}\right)$ in the left-hand side. The results of the previous section imply that this OPE can be interpreted as expanding the state in the Hilbert space $\mathcal{H}$ created by $\chi_{2}^{\dagger}\left(y_{2}\right) \chi_{1}^{\dagger}\left(y_{1}\right)$ in terms of eigenstates of $\left(K^{0}-P^{0}\right) / 2$. This implies that the OPE converges uniformly in $\lambda$ since the norm of $\left|\varphi_{1}\left(x_{1}+\lambda a\right) \varphi_{2}\left(x_{2}+\lambda a\right)\right\rangle$ is independent of $\lambda$ due to translation invariance $\left(a^{0}=0\right.$ !). We can thus use the OPE to approximate $\left\langle\chi_{2}^{\dagger}\left(y_{2}\right) \chi_{1}^{\dagger}\left(y_{1}\right) \varphi_{1}\left(x_{1}+\lambda a\right) \varphi_{2}\left(x_{2}+\lambda a\right)\right\rangle$ for any $\lambda$ to within any $\varepsilon>0$ by a finite sum of 3-point functions of the form $\left\langle\left(\mathcal{D}^{(\alpha)}\right)^{\theta} \mathcal{O}_{i}^{(\nu)}\left(x_{N}\right) \varphi_{1}\left(x_{1}+\lambda a\right) \varphi_{2}\left(x_{2}+\lambda a\right)\right\rangle$ times some $\lambda$-independent coefficients. Of these, only the term corresponding to the identity operator, i.e. the one with $\left(\mathcal{D}^{(\alpha)}\right)^{\theta} \mathcal{O}_{i}^{(\nu)}=1$, does not decay with $\lambda$. It is easily verified that the contribution of this term is precisely equal to $\left\langle\chi_{2}^{\dagger}\left(y_{2}\right) \chi_{1}^{\dagger}\left(y_{1}\right)\right\rangle\left\langle\varphi_{1}\left(x_{1}\right) \varphi_{2}\left(x_{2}\right)\right\rangle$. This finishes the proof of clustering for $m=n=2$.

In the remaining case $m=3, n=1$, we will consider the limit for the integral (since we have not yet proved OS positivity for states involving 3 operators). Note that $\left\langle\varphi_{1}(x)\right\rangle=0$

\footnotetext{
${ }^{32}$ This follows, similarly to $(2.23)$, by applying OS positivity to the state $|\Psi\rangle=\left|\psi\left(F, \varphi_{1} \ldots \varphi_{n}\right)\right\rangle+$ $e^{i \alpha}\left|\psi\left(G, \chi_{1} \ldots \chi_{n}\right)\right\rangle$ where $F, G$ tend to delta functions localizing the operators at points $x_{1}+\lambda a, \ldots, x_{n}+\lambda a$ and $y_{1}^{\theta}, \ldots, y_{n}^{\theta}$ respectively, and choosing the phase $\alpha$ appropriately.
} 
unless $\varphi_{1} \propto 1$, in which case the cluster property becomes trivial. This means that we only need to prove

$$
\lim _{\lambda \rightarrow \infty} \int d x d y \overline{g\left(y_{1}^{\theta}, y_{2}^{\theta}, y_{3}^{\theta}\right)} f\left(x_{1}\right)\left\langle\chi_{3}^{\dagger}\left(y_{3}\right) \chi_{2}^{\dagger}\left(y_{2}\right) \chi_{1}^{\dagger}\left(y_{1}\right) \varphi_{1}\left(x_{1}+\lambda a\right)\right\rangle=0
$$

for $\varphi_{1} \neq 1$. This in turn is a very simple consequence of conformal invariance, and of the fact that $\Delta_{\varphi}>0$ for all operators but the identity. We will be somewhat schematic. The main point is that the configuration $\left(y_{3}, y_{2}, y_{1}, \infty\right)$ is nonsingular from the conformal kinematics point of view (for which the conformal compactification $S_{d}$ of the Euclidean space $\mathbb{R}^{d}$ is the appropriate arena). One way to see it is that the cross ratios are finite in this limit. Thinking in a pedestrian way, we can find a conformal transformation $g_{\lambda}$ which will move points $\left(y_{3}, y_{2}, y_{1}, x_{1}+\lambda a\right)$ to some points which have finite limits as $\lambda \rightarrow \infty$. Transforming the integral (3.23) to this coordinate system, the only singular behavior at large $\lambda$ comes from the Weyl transformation factor as the operator $\varphi_{1}$ is moved from near infinity to a finite position. This factor implies that the integral (3.23) will go to zero as $\lambda^{-2 \Delta_{\varphi}}$, proving clustering in this particular case. See section 6.8.2 for additional details. More generally, the same argument will also work for arbitrary $m$ as long as $n=1$.

\section{$4 \quad$ Euclidean CFT $\Rightarrow$ Wightman: basic strategy}

We will now pass to the main task of our paper: given a Euclidean unitary CFT, recover Minkowski correlators and show that they satisfy Wightman axioms.

Let us first discuss this problem without assuming conformal invariance. Suppose we know correlators $G_{n}^{E}\left(x_{1}, \ldots, x_{n}\right)$ of a scalar field in a Euclidean QFT which is translationally and rotationally invariant, but not necessarily conformally invariant. We are assuming, as discussed above, that the correlators $G_{n}^{E}$ are defined and real-analytic (see footnote 13) for non-coincident Euclidean points $\left(x_{k} \in \mathbb{R}^{d}, x_{i} \neq x_{j}\right)$.

We would like to recover correlators in Minkowski signature. We are only interested here in Wightman correlation functions, where the operator ordering is fixed while the Minkowski time coordinates vary independently. We will call them simply "Minkowski correlators". Starting from this section we will focus on correlators of scalar primaries; correlators of fields in general $\mathrm{SO}(d)$ representations will be considered in our future publication [42].

To understand the equations below, it helps to keep in mind the basic heuristic. If we had a Hilbert space, field operators $\phi$, and a Hamiltonian $H$, then the Minkowski correlators would be given by

$$
\begin{aligned}
G_{n}^{M}\left(x_{1}^{M}, \ldots, x_{n}^{M}\right) & =\left\langle 0\left|\phi\left(0, \mathbf{x}_{1}\right) e^{-i H\left(t_{1}-t_{2}\right)} \phi\left(0, \mathbf{x}_{2}\right) e^{-i H\left(t_{2}-t_{3}\right)} \ldots\right| 0\right\rangle, \\
x_{k}^{M} & =\left(t_{k}, \mathbf{x}_{k}\right) .
\end{aligned}
$$

while the Euclidean correlators by

$$
\begin{aligned}
G_{n}^{E}\left(x_{1}, \ldots, x_{n}\right) & =\left\langle 0\left|\phi\left(0, \mathbf{x}_{1}\right) e^{-H\left(\epsilon_{1}-\epsilon_{2}\right)} \phi\left(0, \mathbf{x}_{2}\right) e^{-H\left(\epsilon_{2}-\epsilon_{3}\right)} \ldots\right| 0\right\rangle, \\
x_{k} & =\left(\epsilon_{k}, \mathbf{x}_{k}\right), \quad \epsilon_{k}>\epsilon_{k+1},
\end{aligned}
$$


We stress that the r.h.s. of these two equations will never be used in this paper. We just use them to illustrate the intuitive property that $G_{n}^{M}$ can be recovered from $G_{n}^{E}$ by analytic continuation $\epsilon_{k} \rightarrow \epsilon_{k}+i t_{k}$ and sending $\epsilon_{k} \rightarrow 0$ while respecting $\epsilon_{k}>\epsilon_{k+1}$. In other words, there is a holomorphic function $G_{n}$ which reduces to $G_{n}^{M}$ in one limit and to $G_{n}^{E}$ in another. The precise domain of analyticity of $G_{n}$ can be clarified from the Wightman axioms [5]. Their basic consequence is that Minkowski correlators can be analytically continued to the "forward tube" (see below), which contains the configuration space of Euclidean timeordered points as a section. In this paper we will derive Wightman axioms, rather than assume them. In particular, we will carry out analytic continuation to the forward tube just from the properties of the Euclidean correlators.

Let us put these observations into a definition of what it means to recover $G_{n}^{M}$ from $G_{n}^{E}$. We consider $n$-point configurations with complexified coordinates:

$$
c=\left(x_{1}, \ldots, x_{n}\right), \quad x_{k}=\left(x_{k}^{0}, \mathbf{x}_{k}\right) \in \mathbb{C}^{d} .
$$

The "forward tube" $\mathcal{T}_{n}$ is defined as the set of all such configurations for which the differences $y_{k}=x_{k}-x_{k+1}=\left(y_{k}^{0}, \mathbf{y}_{k}\right) \in \mathbb{C}^{d}$ satisfy the constraint:

$$
\operatorname{Re} y_{k}^{0}>\left|\operatorname{Im} \mathbf{y}_{k}\right|, \quad k=1, \ldots, n-1 .
$$

Equivalently, this means that vectors $\operatorname{Im}\left(i y_{k}^{0}, \mathbf{y}_{k}\right)$ belong to the open forward light cone of $\mathbb{R}^{1, d-1}$, explaining the name "forward tube". ${ }^{33}$

Let $\mathcal{D}_{n}$ be the subset of the forward tube consisting of the configurations with real spatial parts $\mathbf{x}_{k}$. Equivalently, we have:

$$
\mathcal{D}_{n}=\left\{c \mid x_{k}^{0}=\epsilon_{k}+i t_{k}, \mathbf{x}_{k} \in \mathbb{R}^{d-1}, \epsilon_{1}>\epsilon_{2}>\ldots>\epsilon_{n}\right\} .
$$

Finally, we denote by $\mathcal{D}_{n}^{E}$ the Euclidean part of $\mathcal{D}_{n}$ obtained by setting all $t_{k}=0$.

Minkowski correlators are then defined by the following two-step procedure:

Step 1. One finds an extension $G_{n}^{E}$ from $\mathcal{D}_{n}^{E}$ to a function $G_{n}\left(x_{1}, \ldots, x_{n}\right)$ such that one of the two conditions is satisfied:

$$
G_{n} \text { is defined on } \mathcal{T}_{n} \text {, and holomorphic in all variables } x_{k}^{0}, \mathbf{x}_{k} \text {, }
$$

or

$$
G_{n} \text { is defined on } \mathcal{D}_{n} \text {, is holomorphic in variables } x_{k}^{0} \text { and is real-analytic in } \mathbf{x}_{k} \text {. }
$$

Real analyticity in $\mathbf{x}_{k}$ means that $G_{n}$ can be extended from $\mathcal{D}_{n}$ to a holomorphic function defined on a neighborhood of $\mathcal{D}_{n}$ which allows small imaginary parts for $\mathbf{x}_{k}$. This neighborhood can be arbitrarily small. Condition (4.7) is thus weaker than (4.6) and may be easier to check, although theorem 4.1 below shows that the two conditions are equivalent under the "powerlaw bound" assumption.

\footnotetext{
${ }^{33}$ The just given definition of the forward tube is adapted to the Euclidean coordinates. In section 1.1, eq. (1.2), we wrote the same definition in terms of Minkowski coordinates $x_{k}^{M}=\left(-i x_{k}^{0}, \mathbf{x}_{k}\right)$.
} 
Step 2. Minkowski correlators are defined as the limits of $G_{n}$ from inside $\mathcal{D}_{n}$ by sending $\epsilon_{i} \rightarrow 0:^{34}$

$$
G_{n}^{M}\left(x_{1}^{M}, \ldots, x_{n}^{M}\right)=\lim _{\epsilon_{i} \rightarrow 0} G_{n}\left(x_{1}, \ldots, x_{n}\right), \quad x_{k}^{M}=\left(t_{k}, \mathbf{x}_{k}\right), \quad k=1 \ldots n .
$$

As mentioned several times, Minkowski correlators are expected to be tempered distributions, and therefore this limit has to be understood in the distributional sense. To show that the limit exists and has properties required by Wightman axioms, one relies on the following powerful theorem of several complex variables:

Theorem 4.1 (Vladimirov's theorem). Suppose that the function $G_{n}$ is translation- and rotation-invariant, satisfies (4.7) and in addition satisfies everywhere on $\mathcal{D}_{n}$ the following 'powerlaw bound' with some positive constants $C_{n}, A_{n}, B_{n}$ :

$$
\begin{gathered}
\left|G_{n}\left(x_{1}, \ldots, x_{n}\right)\right| \leqslant C_{n}\left(1+\max _{k} \frac{1}{\epsilon_{k}-\epsilon_{k+1}}\right)^{A_{n}}\left(1+\max _{i}\left|x_{i}-x_{i+1}\right|\right)^{B_{n}} \\
\left|x_{i}-x_{j}\right|^{2} \equiv\left|\epsilon_{i}+i t_{i}-\epsilon_{j}-i t_{j}\right|^{2}+\left|\mathbf{x}_{i}-\mathbf{x}_{j}\right|^{2}
\end{gathered}
$$

Then:

1. Limit (4.8) exists in the sense of tempered distributions. The limiting value $G_{n}^{M}$ is a tempered distribution. ${ }^{35}$

2. The distribution $G_{n}^{M}$ is Poincaré-invariant and satisfies the Wightman spectral condition. I.e. its Fourier transform $W\left(p_{1}, \ldots, p_{n-1}\right)$ with respect to the differences $x_{k}^{M}-x_{k+1}^{M}$ has support in the product of closed forward light cones, which is the region $E_{k} \geqslant\left|\mathbf{p}_{k}\right|, p_{k}=\left(E_{k}, \mathbf{p}_{k}\right)$.

3. The function $G_{n}$ can be extended from a holomorphic function on the whole forward tube $\mathcal{T}_{n}$. The limit (4.8) exists also from the forward tube, i.e. when $\operatorname{Re} y_{k}^{0} \rightarrow$ $0,\left|\operatorname{Im} \mathbf{y}_{k}\right| \rightarrow 0$, satisfying (4.4).

See appendix C for the proof of Vladimirov's theorem and a reminder of what the limit in the sense of distributions means. In the process of the proof, it will be established that the holomorphic function $G_{n}$ on $\mathcal{T}_{n}$ can be written as a "Fourier-Laplace" transform

$$
G_{n}\left(x_{1}, \ldots, x_{n}\right)=\int d p_{1} \ldots d p_{n-1} W\left(p_{1}, \ldots, p_{n-1}\right) e^{\sum_{k=1}^{n-1}\left(-E_{k}\left(x_{k}^{0}-x_{k+1}^{0}\right)+i \mathbf{p}_{k} \cdot\left(\mathbf{x}_{k}-\mathbf{x}_{k+1}\right)\right)}
$$

where $W$ is a tempered distribution which is the Fourier transform of the tempered distribution $G_{n}^{M}$, mentioned in Part 2 of the theorem.

\footnotetext{
${ }^{34}$ We will see in theorem 4.1 that this limit has to be taken along a fixed direction and is independent of direction. If the stronger condition (4.6) holds, the limit can in fact be taken along any direction in the forward null cone.

${ }^{35}$ This part of the theorem does not need translation- and rotation-invariance of $G_{n}$.
} 
To use theorem 4.1, one needs to verify the powerlaw bound (4.9). This strategy was first developed by Osterwalder and Schrader (OS) [2, 3]. ${ }^{36}$ Their full list of assumptions included, in addition to reflection positivity and other OS axioms listed in section 2.2, a less widely known linear growth condition, which roughly says that $G_{n}^{E}$ (appropriately integrated) grows with $n$ not faster than a power of $n$ ! and the degree of its singularities grows not faster than linearly in $n$. The proof of the powerlaw bound was the most technical part of the OS construction, and it crucially relied on the linear growth condition. See appendix 9 for the review.

In this paper we aim to define Minkowski correlators of a conformal field theory, given Euclidean correlators satisfying the CFT axioms of section 2.3. As seen in section 3.1, reflection positivity is robustly encoded in CFT via the positivity requirements for 2-point functions and reality constraints on the OPE coefficients. On the other hand, not much is known about how CFT $n$-point functions grow with $n$. In particular, we are unable to justify the OS growth condition in our setup, hence we cannot appeal to the OS theorem.

In this paper we will be able to circumvent this difficulty, by giving an alternative proof of the powerlaw bound for the most important in applications cases of 2, 3 and 4point functions. Then, by theorem 4.1, these correlators exist in Minkowski space and are tempered distributions. Our proof of the powerlaw bound uses only the Euclidean CFT axioms. In fact, the two- and 3-point function case is almost trivial, these correlators being fixed by conformal invariance. The 4-point function case is much deeper and is one of our main results. Remaining Wightman axioms not mentioned in theorem 4.1 (positivity and clustering) will also be shown to hold.

Remark 4.1. In practice, to compute the Minkowski correlator function one may connect a Minkowski configuration to a Euclidean configuration by a curve $c(s), 0 \leqslant s \leqslant 1$, where $c(0)$ is Euclidean, $c(1)$ Minkowskian, and $c(s), 0<s<1$, belong to the forward tube. In general, the curve should remain in the forward tube except for the endpoint $c(1)$. This means that we must have strict inequalities:

$$
\operatorname{Re} x_{1}^{0}(s)>\operatorname{Re} x_{2}^{0}(s)>\cdots>\operatorname{Re} x_{n}^{0}(s)
$$

everywhere along the analytic continuation contour, except for $s=1$. See figure 1 .

In the literature, one sometimes encounters a different prescription for computing the Minkowski correlators (see e.g. [4], section 3.1), where one puts all points but one at Minkowski positions, and considers correlators as a holomorphic function of the complexified coordinate of the remaining point. One then imagines that Wightman functions are holomorphic functions branching at light-cone separation, and that one can access different operator orderings by going around branch points. We would like to warn the reader that this prescription has to be taken with a grain of salt. To our knowledge there is no general result that the only Wightman functions singularities are branch cuts on the light cones. This is known to be true only in some special cases, e.g. for CFT 2-point and 3-point functions, as well as for CFT 4-point functions in $d=2$ [57]. While some analytic continuation

\footnotetext{
${ }^{36}$ OS used a slightly stronger version of theorem 4.1 with real analyticity in $\mathbf{x}_{k}$ replaced by the weaker assumption of continuity in these variables, but this difference is not essential.
} 


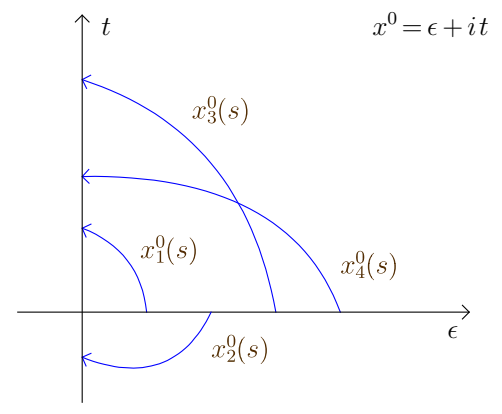

Figure 1. Inequalities (4.12) should be satisfied along the analytic continuation contour.

beyond the forward tube can be done in a general QFT (to the so called permuted extended tube), it does not suffice to justify the analytic continuation prescription of [4] in a general QFT. In CFTs in higher dimensions, the prescription of ref. [4] has some applicability, with the understanding that the correlator is analytic along the continuation contour but may stop being analytic at the endpoint (see appendix A.1).

Remark 4.2. A powerlaw bound in the forward tube (4.9) of course implies a powerlaw bound for the Euclidean 4pt function itself. Together with rotation invariance, this will imply the remaining OS axiom, the "Euclidean temperedness bound" (2.15). Indeed, by rotation invariance, we can choose the direction of the $x^{0}$ axis before applying the Euclidean powerlaw bound. Let us choose the $x^{0}$ direction so that, after ordering the operators according to $\epsilon_{1}>\epsilon_{2}>\epsilon_{3}>\epsilon_{4}$, we have $\epsilon_{k}-\epsilon_{k+1} \geqslant \alpha\left|x_{k}-x_{k+1}\right|$ for each $k$. Such a direction exists for a sufficiently small positive $\alpha$, depending on $d$ and the number of points but not on $x_{i}{ }^{37}$ Applying the Euclidean case of the powerlaw bound (4.9) in this frame we obtain (2.15).

\subsection{Recovering Minkowski averages from Euclidean averages}

Minkowski correlators provided by theorem 4.1, being tempered distributions, can be paired with a Schwartz test function $F$ :

$$
\left(G_{n}^{M}, F\right)=\int d x G_{n}^{M}\left(x_{1} \ldots x_{n}\right) F\left(x_{1} \ldots x_{n}\right) .
$$

Here we will discuss how these pairings can be computed given the Euclidean correlators (compare [2], section 4.3). This discussion will be needed in section 6.7 below and may be skipped on the first reading.

Eq. (4.13) can equivalently be expressed via the Fourier transform $W$ of $G_{n}^{M}$ with respect to $x_{k}-x_{k+1}$ :

$$
\left(G_{n}^{M}, F\right)=\int d p W\left(p_{1}, \ldots, p_{n-1}\right) f\left(p_{1} \ldots p_{n-1}\right),
$$

\footnotetext{
${ }^{37}$ For each pair of points $\left(x_{i}, x_{j}\right)$ we consider the set of direction $\widehat{e}_{0}$ such that $\left|\left(x_{i}-x_{j}\right) \cdot \widehat{e}_{0}\right| \leqslant\left|x_{i}-x_{j}\right| \sin \delta$. This gives a subset $\mathcal{U}_{\delta}$ of the sphere $S^{d-1}$ with $\operatorname{Vol}\left(\mathcal{U}_{\delta}\right) \leqslant 2 \delta \operatorname{Vol}\left(S^{d-2}\right)$. If we choose $\delta_{*}=\frac{\operatorname{Vol}\left(S^{d-1}\right)}{2 n(n-1) \operatorname{Vol}\left(S^{d-2}\right)}$, then the total volume excluded by considering all possible $\left(x_{i}, x_{j}\right)$ pairs is less than $\operatorname{Vol}\left(S^{d-1}\right) / 2$. Therefore, we can find a direction $\widehat{e}_{0}$ such that the opposite inequality $\left|\left(x_{i}-x_{j}\right) \cdot \widehat{e}_{0}\right| \geqslant\left|x_{i}-x_{j}\right| \sin \delta_{*}$ holds for all pairs. Then, renumbering the points in the order of decreasing $x_{i}^{0}$, we obtain $x_{k}^{0}-x_{k+1}^{0} \geqslant\left|x_{k}-x_{k+1}\right| \sin \delta_{*}$.
} 
where $f\left(p_{1} \ldots p_{n-1}\right)=\widehat{F}\left(-p_{1}, p_{1}-p_{2}, \ldots, p_{n-2}-p_{n-1}, p_{n-1}\right)$. Natural pairings for Euclidean correlators are

$$
\left(G_{n}^{E}, \varphi\right)=\int d x G_{n}^{E}\left(x_{1} \ldots x_{n}\right) \Phi\left(x_{1} \ldots x_{n}\right) .
$$

where $\Phi$ is a $C^{\infty}$ test function compactly supported in $x_{1}^{0}>\cdots>x_{n}^{0}$. We wish to discuss how pairings (4.13) or (4.14) can be found given (4.15).

By the Fourier-Laplace representation (4.11), we can write (4.15) as

$$
\left(G_{n}^{E}, \varphi\right)=\int d p W\left(p_{1}, \ldots, p_{n-1}\right) g\left(p_{1}, \ldots, p_{n-1}\right),
$$

where $g$ is any Schwartz class function which agrees inside the forward light cones with $\tilde{\varphi}$, the Fourier-Laplace transform of $\varphi\left(y_{1}, \ldots, y_{n-1}\right)=$ $\int d x_{n} \Phi\left(x_{n}+\sum_{i=1}^{n-1} y_{i}, x_{n}+\sum_{i=2}^{n-1} y_{i}, \ldots, x_{n}\right):$

$$
\begin{gathered}
g \in \mathcal{S}, \quad g(p)=\tilde{\varphi}(p) \quad\left(p_{k} \in \overline{V_{+}}\right) . \\
\tilde{\varphi}\left(p_{1}, \ldots, p_{n-1}\right)=\int d y \varphi\left(y_{1} \ldots y_{n-1}\right) e^{\sum_{k=1}^{n-1}\left(-p_{k}^{0} y^{0}+i \mathbf{p}_{k} \cdot \mathbf{y}_{k}\right)},
\end{gathered}
$$

Note that we cannot just put $g=\tilde{\varphi}$ because $\tilde{\varphi}$ is by itself not a Schwartz function (it may grow exponentially in the negative $p_{k}^{0}$ directions, although it will decrease exponentially in the positive one, since $\varphi$ is supported at $y_{k}^{0}>0$ ). On the other hand the values of $g$ outside the light cones, where $W$ is supported, are unimportant. We can for example take

$$
g\left(p_{1}, \ldots, p_{n-1}\right)=\chi\left(p_{1}^{0}\right) \ldots \chi\left(p_{n-1}^{0}\right) \tilde{\varphi}\left(p_{1}, \ldots, p_{n-1}\right),
$$

where $\chi(s)$ is a $C^{\infty}$ function which equals identically 1 for $s \geqslant 0$ and 0 for $s<-1$.

Suppose then that we find a sequence of $C^{\infty}$ functions $\left\{\varphi_{r}\right\}_{r=1}^{\infty}$ compactly supported at $y_{k}^{0}>0$, the corresponding functions $g_{r} \in \mathcal{S}$ such that $g_{r}=\tilde{\varphi}_{r}$ inside the light cones, and in addition that $g_{r} \rightarrow f$ in the sense of the Schwartz space (i.e. that all Schwartz space seminorms of the difference go to zero), where $f$ is the function in (4.14). Let us put $\Phi_{r}\left(x_{1}, \ldots, x_{n}\right)=\varphi\left(x_{1}-x_{2}, x_{2}-x_{3}, \ldots, x_{n-1}-x_{n}\right) \omega\left(x_{n}\right)$ where $\omega$ is any $C_{0}^{\infty}$ function of integral one. Then we will have

$$
\left(G_{n}^{E}, \Phi_{r}\right)=\left(W, g_{r}\right) \longrightarrow(W, f)=\left(G_{n}^{M}, F\right) \quad(r \rightarrow \infty),
$$

and so we will solve the problem of computing Minkowski averages given Euclidean averages. The following lemma, loosely related to lemma 8.2 in [2], shows that it is indeed possible to find such sequences $\varphi_{r}$ and $g_{r}$ for any Schwartz class $f$.

Lemma 4.2. The set of functions $g \in \mathcal{S}\left(\mathbb{R}^{d(n-1)}\right)$ which satisfy (4.17) for some $\varphi$ a $C^{\infty}$ test function compactly supported in $y_{k}^{0}>0$ is dense in the Schwartz space.

We will give a formal proof; see appendix D for some intuition. We will consider the case $n=2$ as $n>2$ is no more complicated. For each $\varphi$ we consider the set $A_{\varphi}$ of Schwartz functions $g$ which satisfy (4.17):

$$
A_{\varphi}:=\left\{g \in \mathcal{S}\left(\mathbb{R}^{d}\right)|g|_{V_{+}}=\tilde{\varphi}\right\} .
$$


We know that $A_{\varphi}$ is non-empty, e.g. we can take $g$ from (4.18) (it is not hard to show that this is a Schwartz function). Our lemma says that $A \equiv$ "the union of $A_{\varphi}$ over all $\varphi$ " is dense in $\mathcal{S}\left(\mathbb{R}^{d}\right)$. The proof will be by contradiction. Note that $A$ is a linear subspace of $\mathcal{S}\left(\mathbb{R}^{d}\right)$. If $\bar{A} \neq \mathcal{S}\left(\mathbb{R}^{d}\right)$, then there exists a tempered distribution $T \in \mathcal{S}^{\prime}\left(\mathbb{R}^{d}\right)$ such that $T$ vanishes on all test functions from $A$ but does not vanish identically. ${ }^{38}$

So $T$ in particular vanishes on $A_{0}=\left\{g \in \mathcal{S}\left(\mathbb{R}^{d}\right)|g|_{\overline{V_{+}}}=0\right\}$ (take $\varphi=0$ ). This means that the support of the distribution $T$ is contained inside $\overline{V_{+}}$. Consider the Fourier transform of $T$,

$$
\widehat{T}(x):=\int \frac{d^{d} p}{(2 \pi)^{d}} T(p) e^{i p^{0} x^{0}-i \mathbf{p} \cdot \mathbf{x}} .
$$

We can consider $\widehat{T}(x)$ for real $x$ where it is a distribution. Since $\operatorname{supp}(T) \subseteq \overline{V_{+}}$it is also natural to consider $\widehat{T}(\xi+i \eta)$ where $\xi, \eta$ are real and $\eta$ is in the forward cone. We know that $\widehat{T}(x)$ is a holomorphic function for such $x=\xi+i \eta$. We also know that the distribution $\widehat{T}(x)$ for real $x$ can be obtained as a limit of the holomorphic function $\widehat{T}(\xi+i \eta)$ as $\eta \rightarrow 0$.

Let us now come back to the assumption that $(T, g)=0$ for any $g \in A_{\varphi}$. We will apply this to a function $g$ of the form $g=X(p) \tilde{\varphi}$ where $X(p)$ is a $C^{\infty}$ function identically 1 on the forward light cone and such that $X(p) e^{-p^{0} x^{0}+i \mathbf{p} \cdot \mathbf{x}}$ is in Schwartz class for any $x^{0}>0$. It is easy to see that such functions $X(p)$ exist. Writing $(T, g)$ in full we get:

$$
\begin{aligned}
0=(T, g) & =\int d p T(p) X(p) \int d x e^{-p^{0} x^{0}+i \mathbf{p} \cdot \mathbf{x}} \varphi(x) \\
& =\int d x \varphi(x) \int d p T(p) X(p) e^{-p^{0} x^{0}+i \mathbf{p} \cdot \mathbf{x}} \\
& =\int d x \varphi(x) \widehat{T}\left(i x^{0}, \mathbf{x}\right) .
\end{aligned}
$$

The swap of the order of integration between the first and the second line can be justified as follows. Since $T(p)$ is a tempered distribution, we can write it as a finite sum of derivatives of continuous functions of power growth: $T(p)=\sum_{\alpha} \partial_{p}^{\alpha} F_{\alpha}(p)$. Using distributional integration by parts, we can the rewrite the first line of (4.22) as a sum of ordinary integrals, apply Fubini's theorem to swap the integration order, and integrate by parts back to express the answer in terms of $T(p)$.

Because $\varphi(x)$ has compact support in the region $x^{0}>0$, the argument of $\widehat{T}$ in the last line of $(4.22)$ is of the form $\xi+i \eta$ with $\eta=\left(x^{0}, \mathbf{0}\right)$ in the forward light cone, where we know $\widehat{T}$ is analytic. So, from the fact that the last line of (4.22) vanishes for any $\varphi$ we conclude that

$$
\widehat{T}(\xi+i \eta)=0, \quad \xi=(0, \mathbf{x}), \quad \eta=\left(i x^{0}, \mathbf{0}\right), \quad x^{0}>0 .
$$

The set of these points is a totally real submanifold, and so by analyticity we conclude that $\widehat{T}(\xi+i \eta)$ is identically zero for any $\xi \in \mathbb{R}^{d}$ and any $\eta$ in the forward cone. Furthermore,

\footnotetext{
${ }^{38}$ The corresponding statement for normed spaces is standard, being a well-known consequence of the Hahn-Banach theorem (see e.g. [58], Corollary 1.8). For the Schwartz space, we can first find a Schwartz norm $|\cdot|_{n}$, such that $\bar{A}$ is not everywhere dense with respect to this norm, and then apply the standard statement with the norm $|\cdot|_{n}$. This gives a linear functional $T$ on $\mathcal{S}\left(\mathbb{R}^{d}\right)$ continuous with respect to $|\cdot|_{n}$, hence $T \in \mathcal{S}^{\prime}\left(\mathbb{R}^{d}\right)$.
} 
as mentioned above, $\widehat{T}(x)$ for real $x$ is a boundary value of $\widehat{T}(\xi+i \eta)$. Therefore, $\widehat{T}=0$ in the sense of distributions. However we assumed above that $T$ was not identically zero. The reached contradiction shows that $A$ is dense in $\mathcal{S}$.

\section{Two- and 3-point functions}

Let us see how the strategy from section 4 works for the CFT 2-point and 3-point functions. The Euclidean 2-point and 3-point correlators of scalar primaries are given by $\left[x_{i j}^{2}=\left(x_{i}-x_{j}\right)^{2}\right]$

$$
\begin{aligned}
G_{2}^{E}\left(x_{1}, x_{2}\right) & =\frac{1}{\left(x_{12}^{2}\right)^{\Delta}}, \\
G_{3}^{E}\left(x_{1}, x_{2}, x_{3}\right) & =\frac{c_{123}}{\left(x_{12}^{2}\right)^{h_{123}}\left(x_{13}^{2}\right)^{h_{132}}\left(x_{23}^{2}\right)^{h_{23}}}, \quad h_{i j k}=\left(\Delta_{i}+\Delta_{j}-\Delta_{k}\right) / 2 .
\end{aligned}
$$

In this case, the standard way to obtain the Wightman functions is to write these Euclidean correlators in terms of $x_{i j}^{2}$ with $i<j$ (as we did). Substituting the analytic continuation of $x_{i j}^{2}$,

$$
x_{i j}^{2}=\left(x_{i}-x_{j}\right)^{\mu}\left(x_{i}-x_{j}\right)_{\mu}=\left(x_{i}^{0}-x_{j}^{0}\right)^{2}+\left(\mathbf{x}_{i}-\mathbf{x}_{j}\right)^{2}, \quad x_{i}=\left(x_{i}^{0}, \mathbf{x}_{i}\right) \in \mathbb{C}^{d} .
$$

into the Euclidean 2-point and 3-point functions expressions, we obtain their analytic continuations. Suppose further that $x_{i j}^{2} \neq 0, i<j$, in the forward tube (this will be shown below). Then the functions

$$
c \mapsto x_{i j}^{2} \quad(i<j)
$$

are holomorphic functions from the forward tube to $\widetilde{\mathbb{C} \backslash\{0\}}$, the universal covering of the complex plane minus the origin. On the other hand $z \mapsto z^{h}$ is holomorphic from this universal covering to $\mathbb{C}$. Composing these two holomorphic functions, we conclude that $\left(x_{i j}^{2}\right)^{h}, i<j$, are holomorphic on the forward tube. Hence this procedure analytically extends the Euclidean 2-point and 3-point functions to the whole forward tube $\mathcal{T}_{n}(n=2,3)$.

We will now give a simple lemma which proves that indeed $x_{i j}^{2} \neq 0, i<j$, in the forward tube. Actually the lemma says that a bit more is true, namely $x_{i j}^{2} \in \mathbb{C} \backslash(-\infty, 0]$. This has the following practical consequence. In general, to compute the analytic continuation of $(F(c))^{h}$, where $F(c)$ is a nonzero holomorphic function, we need to know the phase of $F(c)$, i.e. to which sheet of the Riemann surface $\widetilde{\mathbb{C} \backslash\{0\}}$ it belongs. To compute the phase we need to connect $c$ to some $c_{E}$ by a curve and analytically continue along this curve, following the phase. However, this is unnecessary for $\left(x_{i j}^{2}\right)^{h}$. Indeed, by the lemma below $x_{i j}^{2}$ always belongs to the principal sheet. So there is no need to use a curve to compute the phase: it can be computed unambiguously just by plugging the coordinates into (5.3).

Lemma 5.1. Let $y=\left(y^{0}, \mathbf{y}\right) \in \mathbb{C}^{d}$ satisfy $\operatorname{Re} y^{0}>|\operatorname{Im} \mathbf{y}|$. Then

$$
y^{2} \equiv\left(y^{0}\right)^{2}+\mathbf{y}^{2} \in \mathbb{C} \backslash(-\infty, 0] .
$$


Proof. We will denote by Greek letters $\xi, \eta$, etc., vectors of Minkowski space $\mathbb{R}^{1, d-1}$ with the Minkowski inner product $\xi^{2}=-\left(\xi^{0}\right)^{2}+\boldsymbol{\xi}^{2}{ }^{39}$ Decomposing the vector $\left(i y^{0}, \mathbf{y}\right)$ into its real and imaginary parts:

$$
\left(i y^{0}, \mathbf{y}\right)=\xi+i \eta,
$$

condition $\operatorname{Re} y^{0}>|\operatorname{Im} \mathbf{y}|$ means that $\eta^{0}>0$ and $-\eta^{2}>0$, i.e. $\eta$ is in the open forward light cone, which we will denote by $\eta \succ 0$. In this notation, we have to prove that

$$
(\xi+i \eta)^{2}=\xi^{2}-\eta^{2}+2 i(\xi \eta) \notin \mathbb{C} \backslash(-\infty, 0] .
$$

where by our conventions all inner products involving $\xi, \eta$ are Minkowski. Suppose this is violated, i.e.

$$
(\xi \eta)=0, \quad \xi^{2}-\eta^{2}<0,
$$

for some $\xi, \eta$. Since $\eta$ is timelike, $(\xi \eta)=0$ implies that $\xi$ is spacelike. But then $\xi^{2}-$ $\eta^{2}=\xi^{2}+\left(-\eta^{2}\right)>0$. Thus the two conditions in (5.8) cannot both be true, and (5.7) is proved.

As the next step of implementing the strategy from section 4, we need to check that the constructed analytic continuations satisfy a powerlaw bound so that we can apply theorem 4.1. Although we already constructed analytic continuation to the whole $\mathcal{T}_{n}$, we only need to check the bound on $\mathcal{D}_{n}$ which is somewhat easier. The powerlaw bound follows from the following lemma.

Lemma 5.2. (a) Let $y=(\varepsilon+i s, \mathbf{y}), \varepsilon, s \in \mathbb{R}, \mathbf{y} \in \mathbb{R}^{d-1}$. Then $y^{2}$ is bounded above and below in the absolute value, as follows:

$$
\varepsilon^{2} \leqslant\left|y^{2}\right| \leqslant|y|^{2} \equiv|\varepsilon+i s|^{2}+\mathbf{y}^{2}
$$

(b) On $\mathcal{D}_{n}(n=2,3)$, each $1 /\left(x_{i j}^{2}\right)^{h}$ factor in (5.1), (5.2) (h $\left.\in \mathbb{R}\right)$ satisfies a powerlaw bound:

$$
\left|\frac{1}{\left(x_{i j}^{2}\right)^{h}}\right| \leqslant \frac{\left|x_{i}-x_{j}\right|^{B}}{\left(\epsilon_{i}-\epsilon_{j}\right)^{A}} \quad(i>j)
$$

where $A=2 h, B=0$ for $h$ positive and $A=0, B=-2 h$ for $h$ negative.

Proof. (a) The upper bound is obvious. Let us show the lower bound by an explicit computation (see lemma 6.3 below for an alternative proof). We have:

$$
\left|y^{2}\right|^{2} \equiv\left|(\varepsilon+i s)^{2}+\mathbf{y}^{2}\right|^{2}=\left(\varepsilon^{2}-s^{2}+\mathbf{y}^{2}\right)^{2}+4 \varepsilon^{2} s^{2},
$$

Minimizing this in $\mathbf{y}$, we get

$$
\min _{\mathbf{y}}\left|y^{2}\right|^{2}= \begin{cases}\left(\varepsilon^{2}-s^{2}\right)^{2}+4 \varepsilon^{2} s^{2}=\left(\varepsilon^{2}+s^{2}\right)^{2}, & |s| \leqslant \varepsilon \\ 4 \varepsilon^{2} s^{2}, & |s| \geqslant \varepsilon .\end{cases}
$$

Minimizing this next in $s$, we find that the absolute minimum is located at $\mathbf{y}=0, s=0$, and is equal to $\varepsilon^{4}$. Part (b) follows from (a).

\footnotetext{
${ }^{39}$ We remind the readers that everywhere in this paper we are using $-,+, \ldots,+$ Minkowski signature.
} 
Now that we have the powerlaw bound, we can apply theorem 4.1. We conclude that the Minkowski 2-point and 3-point functions, defined as $\epsilon_{i} \rightarrow 0$ limits of the analytically continued Euclidean correlators, exist, are Lorentz-invariant tempered distributions, and satisfy the spectral condition. ${ }^{40}$

\subsection{Comparison with the $i \varepsilon$-prescription}

Here we will comment on the " $i \varepsilon$-prescription" often used in the literature to define Minkowski 2-point and 3-point correlators, and how it compares with our definition. We will focus on the 2-point case for definiteness (same remarks hold for the 3-point case).

The $i \varepsilon$-prescription defines the Minkowski 2-point correlator $G_{2}^{M}\left(x_{1}^{M}, x_{2}^{M}\right)$ as

$$
\frac{1}{\left(-(s-i \varepsilon)^{2}+\mathbf{y}^{2}\right)^{\Delta}}
$$

with $s=t_{1}-t_{2}, \mathbf{y}=\mathbf{x}_{1}-\mathbf{x}_{2}$ and taking the $\varepsilon \rightarrow 0^{+}$limit. The precise meaning of the limit is often left implicit in the physics literature. Away from the light cone the 2-point correlator is an ordinary function, the limit can be understood pointwise and it agrees with our definition. Clearly, on the light cone the limit must be understood in distributional sense, integrating against a test function $f(s, \mathbf{y})$. That is what we showed above: Vladimirov's theorem guarantees that the limit $\epsilon \rightarrow 0$ exists as a tempered distribution and can be therefore integrated against any Schwartz test function. In physics literature, one instead often hears that such integrals should be defined by "shifting the integration contour". Note however that this alternative way of understanding the $\epsilon \rightarrow 0$ limit would only work for analytic test functions. Let us discuss the consequences of this limitation.

It is helpful to recall that the theory of distributions commonly uses three classes of test functions, denoted $\mathcal{S}, \mathcal{K}, \mathcal{Z}$ [61]. Here $\mathcal{S}$ is the space of Schwartz functions, $\mathcal{K}$ (denoted sometimes by $\mathcal{D}$ ) is the space of compactly supported $C^{\infty}$ functions, and $\mathcal{Z}$ consists of entire holomorphic functions decreasing faster than any power in the real directions and bounded by some fixed exponential in the imaginary directions. Note that $\mathcal{K}, \mathcal{Z} \subset \mathcal{S}$. The corresponding distribution spaces thus satisfy the opposite inclusion: $\mathcal{S}^{\prime} \subset \mathcal{K}^{\prime}, \mathcal{Z}^{\prime}$. The elements of $\mathcal{S}^{\prime}$ are precisely the tempered distributions discussed above, $\mathcal{K}^{\prime}$ are distributions on the compactly supported test functions ${ }^{41}$, while $\mathcal{Z}^{\prime}$ is yet another distributional class.

Importantly, the Fourier transform $\mathcal{F}$ leaves $\mathcal{S}$ invariant. Since the Fourier transform is defined in the theory of distributions by duality, we also have $\mathcal{F}\left(\mathcal{S}^{\prime}\right)=\mathcal{S}^{\prime}$ : the Fourier transform of a tempered distribution is also a tempered distribution. On the other hand, one can show (see [61]) that $\mathcal{F}(\mathcal{K})=\mathcal{Z}$. This is the rationale behind introducing the space $\mathcal{Z}$, and this also implies that $\mathcal{F}$ maps $\mathcal{K}^{\prime}$ to $\mathcal{Z}^{\prime}$ and vice versa.

Coming back to (5.12), shifting the integration contour defines this distribution as an element of $\mathcal{Z}^{\prime}$. The pairing with a test function $f \in \mathcal{Z}$ is thus defined by

$$
\int_{C} d z \int d \mathbf{y} \frac{1}{\left(-z^{2}+\mathbf{y}^{2}\right)^{\Delta}} f(z, \mathbf{y})
$$

\footnotetext{
${ }^{40}$ Since these are tempered distributions, their Fourier transforms are well defined. Explicit expressions for these Fourier transforms are known in many cases. See [59] for $\left\langle\mathcal{O}_{\Delta, l}(p) \mathcal{O}_{\Delta, l}(-p)\right\rangle$ and [60] for $\left\langle\mathcal{O}_{\Delta_{1}}\left(p_{1}\right) \mathcal{O}_{\Delta_{2}}\left(p_{2}\right) \mathcal{O}_{\Delta, l}\left(p_{3}\right)\right\rangle$.

${ }^{41}$ They are briefly mentioned in the proof of theorem 4.1, appendix C.2, after eq. (C.13).
} 
with the contour $C$ running parallel to the real axis in the lower half plane. ${ }^{42}$ By the previous paragraph, this is then sufficient to define the Fourier transform of the 2-point function as an element of $\mathcal{K}^{\prime}$. By moving the contour far away from the real axis, one shows that the Fourier transform vanishes for negative energies, and by Lorentz invariance one concludes that the support must belong to the forward light cone. These arguments have parallels in the proof of Part 2 of theorem 4.1 (see appendix C.2).

Compared to this simple and almost elementary discussion, theorem 4.1 proves a stronger statement that the 2-point distribution (5.12) can be extended to test functions of Schwartz class and, furthermore, to functions which have only a finite number of derivatives as expressed by eq. (C.3). This can be seen as a finer characterization of the singularity structure at short distances. The Fourier transform is then also a tempered distribution, thus bounded by some power, which is a stronger statement than it being an element of $\mathcal{K}^{\prime}$ since those can grow arbitrarily fast at infinity.

Since the 2-point and 3-point correlators are known in closed form, one can in principle verify that their Fourier transform does not grow too fast at infinity by an explicit computation. This would provide an alternative proof of temperedness. Our point here is that theorem 4.1 reaches this conclusion without any computations. For the 4-point correlators considered below, the Fourier transform cannot be evaluated easily, and theorem 4.1 appears to be the only realistic way to show temperedness.

It is instructive to discuss why we insist so much on temperedness. In other words, why Wightman axioms require that the Minkowski $n$-point correlators must be tempered distributions, and not of some other class? There is a simple reason why temperedness is a natural requirement, while $\mathcal{K}^{\prime}$ or $\mathcal{Z}^{\prime}$ would not suffice. The point is that Wightman axioms include both commutativity at spacelike separation and the spectral condition (the Fourier transform supported in the forward tube). Both these conditions need compactly supported test functions: the former in position space, the latter in momentum space. The space $\mathcal{S}$ is large enough to write both these conditions, while $\mathcal{K}$ or $\mathcal{Z}$ are inadequate as we would lose one of them. ${ }^{43}$

Finally, sometimes by the $i \varepsilon$-prescription one means the following simplified form of (5.12):

$$
\frac{1}{\left(-s^{2}+i 0^{+} \operatorname{sign}(s)+\mathbf{y}^{2}\right)^{\Delta}}
$$

which agrees with (5.12) away from the light cone. By Vladimirov's theorem, this defines a distribution for $s>0$ (including the light cone) and another distribution for $s<0$, but it is not an adequate starting point for defining the distribution around $(s, \mathbf{y})=(0,0)$.

\footnotetext{
${ }^{42}$ We can somewhat relax the condition $f \in \mathcal{Z}$. At the very least, $f$ must be holomorphic in the lower half-plane close to the real axis and decrease sufficiently fast at infinity for the integral to be convergent.

${ }^{43}$ For completeness it should be noted that one can reduce $\mathcal{S}$ a bit and still be able to formulate both these axioms, as for Jaffe fields [62], which may have stronger-than-powerlaw singularities at short distances. For CFTs and for any theory which asymptotes to a CFT at short distances, there is no reasons to consider such modifications, and $\mathcal{S}$ remains the natural choice.
} 


\section{Scalar 4-point function}

This section is the heart of our paper. In it we will show how to define Minkowski 4-point functions starting from Euclidean 4-point functions of a unitary CFT. We will follow the strategy of section 4 and in particular will rely on theorem 4.1. To avoid inessential details, we will focus on the case of four identical scalars. Non-identical scalars can be treated by the same argument (see section 6.10). Additional complications arise for spinning operators; this case is postponed to a future publication [42].

So, we consider the Euclidean CFT 4-point function of four identical scalar Hermitian primaries, which by conformal invariance can be written as:

$$
G_{4}^{E}\left(c_{E}\right) \equiv\left\langle\mathcal{O}\left(x_{1}\right) \mathcal{O}\left(x_{2}\right) \mathcal{O}\left(x_{3}\right) \mathcal{O}\left(x_{4}\right)\right\rangle=\frac{1}{\left(x_{12}^{2} x_{34}^{2}\right)^{\Delta_{\mathcal{O}}}} g\left(c_{E}\right) .
$$

Here $c_{E}=\left(x_{1}, x_{2}, x_{3}, x_{4}\right)$ denotes an ordered configuration of four Euclidean non-coincident points $\left(x_{k} \in \mathbb{R}^{d}, \quad x_{i} \neq x_{j}\right), \Delta_{\mathcal{O}}$ is the scaling dimension of $\mathcal{O}$, and $g\left(c_{E}\right)$ is a real function which depends only on the conformal class of $c_{E}$. It can be written as a function of two conformally invariant cross-ratios $u, v$ :

$$
g\left(c_{E}\right)=g(u, v), \quad u=\frac{x_{12}^{2} x_{34}^{2}}{x_{13}^{2} x_{24}^{2}}, \quad v=\frac{x_{14}^{2} x_{23}^{2}}{x_{13}^{2} x_{24}^{2}} .
$$

Our plan is as follows. After a discussion of the basic issues involved in the analytic continuation of the 4-point function (section 6.1), we will introduce a representation in terms of the radial coordinates $\rho, \bar{\rho}$ (section 6.2), and use it to construct the analytic continuation to the whole forward tube $\mathcal{T}_{4}$ (section 6.3 ). This construction works because $\rho, \bar{\rho}$ remain strictly inside the unit disc everywhere in the forward tube (lemma 6.1 and eq. (6.21)), a fundamental fact proved in section 6.4. We then briefly review the well-established powerlaw bound on $g(\rho, \bar{\rho})$ with respect to $\rho, \bar{\rho}$, and prove a powerlaw bound on $|\rho(c)|,|\bar{\rho}(c)|$ with respect to $c \in \mathcal{T}_{4}$. Combining these powerlaw bounds together, we will get a powerlaw bound on the analytically continued 4-point function $G_{4}(c)$, which by theorem 4.1 implies (as $c$ approaches the Minkowski region) the existence of the boundary limit of $G_{4}(c)$ as a tempered distribution (section 6.5). After establishing temperedness, we will derive the Minkowski conformal invariance (section 6.6), Wightman positivity (section 6.7), Wightman clustering (section 6.8) and local commutativity (section 6.9). Some of them do not rely on conformal properties: for these we will use the standard arguments given by Osterwalder and Schrader [2]. In section 6.10, we will generalize the above results to non-identical scalars by using Cauchy-Schwarz arguments.

\subsection{Informal introduction to basic issues}

Here we wish to outline a few basic difficulties which must be dealt with when analytically continuing the 4-point function. We will be using $u, v$ coordinates as an example, although we will see below that other coordinates will be more suitable for our task. Readers uninterested in philosophical discussions may skip directly to section 6.2.

Given any point $c$ of the forward tube, we can connect it to a Euclidean point $c_{E}$ by a curve, and analytically continue the 4-point function along the curve (see figure 2, left). 


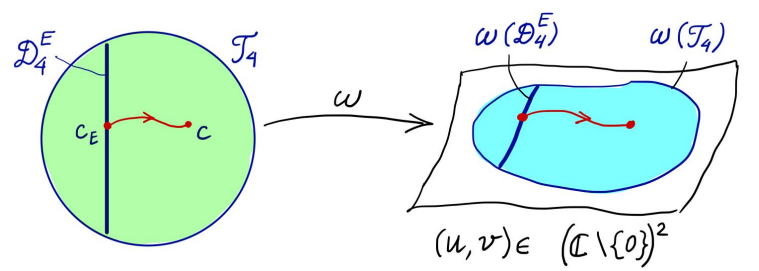

Figure 2. Illustration of the discussion in section 6.1 .

The forward tube being simply connected, the analytic continuation (if it exists) does not depend on the curve. Furthermore, let us take into account that our conformal 4-point function effectively only depends on two variables $u, v$. Applying lemma 5.1, we see that $u, v$ are both nonzero holomorphic functions on the forward tube. Consider the map:

$$
\omega: c \rightarrow(u, v)
$$

Since the forward tube is simply connected, we can consider this map as acting from $\mathcal{T}_{4}$ to $(\widetilde{\mathbb{C} \backslash\{0\}})^{2}$, where tilde denotes the universal cover. Denote by $\omega\left(\mathcal{T}_{4}\right)$ and $\omega\left(\mathcal{D}_{4}^{E}\right)$ the images of the forward tube and of its Euclidean part under this map (see figure 2, right).

Now suppose that we found an analytic continuation of $g(u, v)$ from $\omega\left(\mathcal{D}_{4}^{E}\right)$, where it is initially defined, to the whole of $\omega\left(\mathcal{T}_{4}\right)$. Then we could immediately write down the analytic continuation of the 4-point function to the forward tube as follows: ${ }^{44}$

$$
G_{4}(c)=\frac{1}{\left(x_{12}^{2} x_{34}^{2}\right)^{\Delta_{\mathcal{O}}}} g(u(c), v(c)) .
$$

This formula defines the analytic continuation to the forward tube as a composition of two holomorphic functions:

$$
\mathcal{T}_{4} \stackrel{\omega}{\rightarrow} \omega\left(\mathcal{T}_{4}\right) \stackrel{g}{\rightarrow} \mathbb{C}
$$

We would like to use this strategy, but its direct implementation is hindered by a couple of difficulties:

- We don't know much about the shape or even topology of $\omega\left(\mathcal{T}_{4}\right)$. E.g. we don't know if this set is simply connected. The continuous image of a simply connected set, such as the forward tube, does not have to be simply connected (figure 3 ). If $\omega\left(\mathcal{T}_{4}\right)$ is not simply connected, there is no guarantee that $g(u, v)$ will be single-valued on it. And if $g(u, v)$ has branch cuts, then a simple formula like (6.4) using only the endpoint values $(u(c), v(c))$ will not work; we will need to know in addition "from which side of the cut" we got to this point along the analytic continuation contour (see figure 3 ).

- To be sure, we don't know if the above difficulty is actually realized. Perhaps the set $\omega\left(\mathcal{T}_{4}\right)$ is, after all, simply connected, and $g(u, v)$ has a single-valued analytic continuation to it. Even if this is the case, how can we construct this extension starting from $g(u, v)$ in the Euclidean region? 


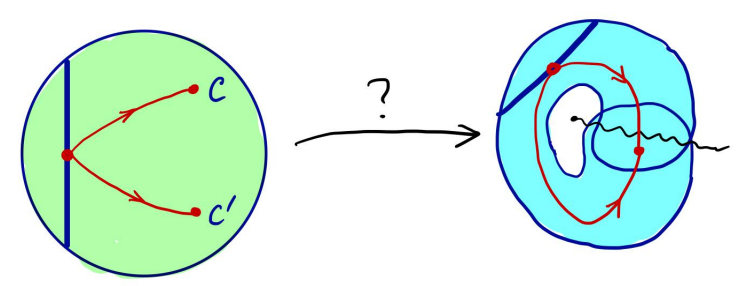

Figure 3. Illustration of a potential difficulty if the set $\omega\left(\mathcal{T}_{4}\right)$ were not simply connected (see section 6.1).

In this paper we will circumvent these difficulties rather than attacking them head-on. In the Euclidean region, one often uses different variables to parametrize the cross-ratios $u, v$, such as the Dolan-Osborn variables $z, \bar{z}$, or the radial variables $\rho, \bar{\rho}$. As one can imagine, a smart choice of Euclidean variables can greatly simplify the analytic continuation. We will see that the radial variables are ideally suited for this task, allowing a natural resolution of the above-mentioned difficulties.

\subsection{Euclidean 4-point function in radial coordinates}

We first recall the well-known Dolan-Osborn variables $z, \bar{z}[63,64]$, which are two complex variables related to $u, v$ by

$$
\begin{aligned}
u & =z \bar{z}, \quad v=(1-z)(1-\bar{z}), \quad \text { or } \\
z, \bar{z} & =\frac{1}{2}\left(1+u-v \pm \sqrt{(1+u-v)^{2}-4 u}\right) .
\end{aligned}
$$

Since in the Euclidean case we only consider non-coincident points, we have $u, v \neq 0$, and hence $z, \bar{z} \neq 0,1$. It is possible to fix a Euclidean conformal frame by setting the four points to positions

$$
x_{1}=0, \quad x_{2}=a \widehat{e}_{0}+b \widehat{e}_{1}, \quad x_{3}=\widehat{e}_{0}, \quad x_{4}=\infty \widehat{e}_{0},
$$

where $\widehat{e}_{\mu}$ is the standard orthonormal basis of $\mathbb{R}^{d}$. Using this frame, we obtain $z, \bar{z}=a \pm i b$. This shows that in the Euclidean, the variables $z, \bar{z}$ are complex-conjugate $\left(\bar{z}=z^{*}\right)$.

Euclidean configurations with real $z=\bar{z}$ correspond to four points lying on a circle, which maps in the frame (6.8) to four points on a line. The three possibilities $z<0$, $z \in(0,1), z \in(1,+\infty)$ are then realized for different cycling orderings.

The radial variables $\rho, \bar{\rho} \in \mathbb{C}[65,66]$ are defined in terms of the Dolan-Osborn variables by the formula:

$$
\rho=f(z), \quad \bar{\rho}=f(\bar{z}), \quad f(w):=\frac{w}{(1+\sqrt{1-w})^{2}} .
$$

The function $f(w)$ in this definition ${ }^{45}$ is the uniformization map for the complex plane minus the cut $(1,+\infty)$, i.e. it is a one-to-one map of $\mathbb{C} \backslash[1,+\infty)$ onto the unit disk. Eq. (6.9)

\footnotetext{
${ }^{44}$ The prefactor analytically continues just as the 2-point and 3-point functions in section 5 .

${ }^{45}$ The definition assumes the standard branch of the square root function.
} 
thus associates with any Euclidean configuration a pair of complex conjugate $\rho, \bar{\rho}\left(\bar{\rho}=\rho^{*}\right)$ belonging to the unit disk: $|\rho| \leqslant 1$. Moreover we have $|\rho|<1$ except for the Euclidean configurations with $z=\bar{z} \in(1,+\infty)$. As explained above, this happens when four points lie on a circle in the cyclic order 1324 . For such exceptional configurations one may define $\rho, \bar{\rho}$ by continuity so that $|\rho|=1, \bar{\rho}=\rho^{*}$.

The meaning of the coordinate $\rho$ is clarified by mapping the 4 -point configuration to a conformal frame (compare (6.8))

$$
x_{1}=-\alpha \widehat{e}_{0}-\beta \widehat{e}_{1}, \quad x_{2}=\alpha \widehat{e}_{0}+\beta \widehat{e}_{1}, \quad x_{3}=\widehat{e}_{0}, \quad x_{4}=-\widehat{e}_{0},
$$

Using this frame, we obtain $\rho, \bar{\rho}=\alpha \pm i \beta$.

There is a small difference between $d=2$ and $d \geqslant 3$ dimensions. In $d \geqslant 3$, conformal frames (6.8) and (6.10) are unique only up to a sign of $b$ and $\beta$ (flipped rotating by $\pi$ in the 12 plane), which implies that pairs $(z, \bar{z})$ and $(\rho, \bar{\rho})$ are defined only up to permutation. On the other hand in $d=2$ flipping the sign of $b$ or $\beta$ is a parity transformation, which is not in the identity component of the conformal group. Hence the conformal frames are unique and $z, \bar{z}$ as well as $\rho, \bar{\rho}$ are individually meaningful.

In a unitary Euclidean CFT, the 4-point function admits a power-series expansion in the $\rho$ coordinate, absolutely convergent whenever $|\rho|<1$ [65]. Specifically, the function $g\left(c_{E}\right)$ appearing in the 4-point function (6.1) of four identical scalar Hermitean primaries has a series expansion of the form

$$
g\left(c_{E}\right)=\sum_{\delta, m} p_{\delta, m} r^{\delta} e^{i m \theta}
$$

where the sum runs over a discrete set of pairs $(\delta, m)$ with $\delta \geqslant 0, m \in 2 \mathbb{Z}$, and the variables $r, \theta \in \mathbb{R}$ are the modulus and the phase of $\rho\left(c_{E}\right)=r e^{i \theta}$. The sum is absolutely convergent when $r=\left|\rho\left(c_{E}\right)\right|<1$. In addition, we know that $|m| \leqslant \delta$ and $p_{\delta, m} \geqslant 0$ for all terms in (6.11). Finally, when $d \geqslant 3$ we have $p_{\delta,-m}=p_{\delta, m}$, so that the r.h.s. of (6.11) is uniquely defined in spite of $\rho\left(c_{E}\right)$ being defined only up to complex conjugation.

The readers familiar with this fact may skip to section 6.3 where we will use it to perform analytic continuation. In the rest of this section we recall how it follows from the CFT axioms [65, 67].

We consider the 4-point function in the conformal frame configuration (6.10) and write it as the inner product of two states created by the operators outside and inside a unit sphere $S$ centered at the origin:

$$
\langle\mathcal{O}(1,0, \mathbf{0}) \mathcal{O}(-1,0, \mathbf{0}) \mid \mathcal{O}(\alpha, \beta, \mathbf{0}) \mathcal{O}(-\alpha,-\beta, \mathbf{0})\rangle
$$

We can find a conformal transformation which maps the sphere $S$ to $x^{0}=0$ plane, its center 0 to $x_{S}$ and the infinity to $x_{N}$. This is the setup in which we developed the CFT Hilbert space picture in section 3.2. Applying the inverse transformation, we are allowed to use the Hilbert space language in the frame (6.12), which is the familiar setting of radial quantization. We decompose the radial quantization Hilbert space, produced by local 
operators inserted at the origin, in orthonormalized eigenstates $|\delta, m\rangle$ of the dilatation $D$ and the planar rotation $M_{01}$. The ket state is expanded in this basis as

$$
|\mathcal{O}(\alpha, \beta, \mathbf{0}) \mathcal{O}(-\alpha,-\beta, \mathbf{0})\rangle=\sum_{\delta, m} c_{\delta, m} r^{\delta-2 \Delta_{\varphi}} e^{i m \theta}|\delta, m\rangle .
$$

The dependence of the expansion coefficients in this formula on $r$ and $\theta$ is fixed by knowing how the state in the l.h.s. transforms under rotations and dilatations. The transformation $\theta \rightarrow \theta+\pi$ swaps the two operators leaving the state invariant for the considered case of identical operators. Hence the state in the r.h.s. also must remain invariant, proving that $m$ must be even.

Setting $r=1, \theta=0$ in (6.13), we get

$$
|\mathcal{O}(1,0, \mathbf{0}) \mathcal{O}(-1,0, \mathbf{0})\rangle=\sum_{\delta, m} c_{\delta, m}|\delta, m\rangle .
$$

In the considered frame the OS reflection is the inversion with respect to the sphere $S$ : $x^{\mu} \rightarrow x^{\mu} / x^{2}$. In particular, this leaves $x_{3}$ and $x_{4}$ invariant. Applying this transformation to $(6.14)$, we get

$$
\langle\mathcal{O}(1,0, \mathbf{0}) \mathcal{O}(-1,0, \mathbf{0})|=\sum_{\delta, m} c_{\delta, m}^{*}\langle\delta, m| .
$$

Taking the inner product of (6.13) and (6.15), we get

$$
\langle\mathcal{O}(1,0, \mathbf{0}) \mathcal{O}(-1,0, \mathbf{0}) \mid \mathcal{O}(\alpha, \beta, \mathbf{0}) \mathcal{O}(-\alpha,-\beta, \mathbf{0})\rangle=\sum_{\delta, m}\left|c_{\delta, m}\right|^{2} r^{\delta-2 \Delta_{\varphi}} e^{i m \theta}
$$

Comparing this with eq. (6.1), and using that $x_{12}^{2}=4 r^{2}, x_{34}^{2}=4$ in the considered conformal frame, we obtain (6.11) with $p_{\delta, m}=16^{\Delta_{\mathcal{O}}}\left|c_{\delta, m}\right|^{2} \geqslant 0$.

In the above argument we chose for simplicity the sphere of radius 1 , but any sphere of radius $r<r_{0}<1$ would work equally well and give rise to the same expression. Absolute convergence for $r<1$ follows, because both the bra and the ket states are normalizable for such $r_{0}$ (while for $r_{0}=1$ as above the bra state $\left\langle\varphi\left(x_{3}\right) \varphi\left(x_{4}\right)\right|$ is not normalizable).

The restriction $|m| \leqslant \delta$ follows from the $2 \mathrm{~d}$ unitarity bounds. The $2 \mathrm{~d}$ unitarity bound applies, as any $d$-dimensional CFT restricted to a plane can be seen as a unitary $2 \mathrm{~d}$ CFT. For 2 d primaries of spin $J$ and dimension $\Delta$, the 2 d unitarity bound says $|J| \leqslant \Delta$. The descendants at level $n \in \mathbb{Z}_{\geqslant 0}$ have $\delta=\Delta+n,|m-J| \leqslant n$, hence $|m| \leqslant \delta$ follows.

Finally, let us prove that $p_{\delta, m}=p_{\delta,-m}$ in $d \geqslant 3$. We consider eq. (6.14) and perform a $\pi$ rotation in the 12 plane. In the r.h.s. $|\delta, m\rangle \rightarrow|\delta,-m\rangle$ because $M_{01} \rightarrow-M_{01}$ under such a rotation. On the other hand the l.h.s. does not change. This implies that we must have $c_{\delta, m}=c_{\delta,-m}$, and hence $p_{\delta, m}=p_{\delta,-m}$. (In $d=2$, these properties also hold under the additional assumption of parity invariance.)

\subsection{Analytic continuation}

In this section we will construct the analytic continuation of the Euclidean 4-point function (6.1) to the forward tube $\mathcal{T}_{4}$ (recall the forward tube definition (4.4)). Analytic continuation to $\mathcal{D}_{4} \subset \mathcal{T}_{4}$ has already been given in [37], section 3.4, and we will use a somewhat 
streamlined version of that construction. We will analytically continue to the full forward tube $\mathcal{T}_{4}$, since this does not lead to additional complications.

The analytic continuation will be given by the formula

$$
G_{4}(c)=\frac{1}{\left(x_{12}^{2} x_{34}^{2}\right)^{\Delta_{\mathcal{O}}}} g(c), \quad c \in \mathcal{T}_{4} .
$$

Here the prefactor trivially analytically continues to $\mathcal{T}_{4}$ similarly to the 2-point and 3-point functions discussed in section 5. We will construct $g(c)$, analytic continuation of $g\left(c_{E}\right)$, starting from eq. (6.11).

First we have to define the variables $z(c), \bar{z}(c)$ on the forward tube, which is naturally done as follows. Given a configuration $c \in \mathcal{T}_{4}$, we evaluate $u=u(c), v=v(c)$ via (6.2). By lemma 5.1, $u(c)$ and $v(c)$ are nonzero holomorphic functions on the forward tube. We then define $z(c), \bar{z}(c)$ via $(6.7)$ :

$$
z(c), \bar{z}(c)=\frac{1}{2}\left(1+u(c)-v(c) \pm \sqrt{[1+u(c)-v(c)]^{2}-4 u(c)}\right) .
$$

Unlike for Euclidean configurations, for a general configuration $c \in \mathcal{T}_{4}$ these are two complex numbers unrelated by conjugation. Since $u(c)$ and $v(c)$ are nonzero, eq. (6.6) implies $z(c), \bar{z}(c) \in \mathbb{C} \backslash\{0,1\}$.

Since eq. (6.18) only defines $z(c), \bar{z}(c)$ up to permutation, we view it as a map from the forward tube to $\mathbb{C}^{2} / \mathbb{Z}_{2}$, the set of unordered pairs of complex numbers. This map is continuous, and is analytic everywhere except on $\Gamma \subset \mathcal{T}_{4}$ where the expression under the square root vanishes:

$$
\Gamma=\left\{c \in \mathcal{T}_{4}:[1+u(c)-v(c)]^{2}-4 u(c)=0\right\} .
$$

Actually, it turns out that in $d=2$ one can resolve the ambiguity inherent in eq. (6.18) and define $z(c), \bar{z}(c)$ as individually globally holomorphic functions on $\mathcal{T}_{4}$. We will bring up this fact below when we need it. Ref. [37], appendix A, showed that such an improvement is impossible in $d \geqslant 3$.

The following result is fundamental for our construction. The proof is elementary but a bit tricky and is postponed to section 6.4.

Lemma 6.1. For any $c \in \mathcal{T}_{4}$ we have $z(c), \bar{z}(c) \notin[1,+\infty)$.

We next define $\rho(c), \bar{\rho}(c)$ on $\mathcal{T}_{4}$, via

$$
\rho(c)=f(z(c)), \quad \bar{\rho}(c)=f(\bar{z}(c)),
$$

where $f$ is the same function as in (6.9), mapping $\mathbb{C} \backslash[1,+\infty)$ onto the unit disk. By lemma 6.1, we then have ${ }^{46}$

$$
0<|\rho(c)|,|\bar{\rho}(c)|<1 \quad \text { for any } \quad c \in \mathcal{T}_{4} .
$$

Moreover, $\rho(c)$ and $\bar{\rho}(c)$ are locally holomorphic away from $\Gamma$. Because of this, and since eq. (6.11) for the 4-point function converges in the Euclidean for any $|\rho|<1$, we may hope

\footnotetext{
${ }^{46}$ Note that the converse is not true: the region in which $0<|\rho|,|\bar{\rho}|<1$ is larger than the forward tube. For example, it includes the extended forward tube (see section 6.9.1).
} 
to use eq. (6.11) to analytically continue $g(c)$ to the whole forward tube. We will now carry out this strategy. Note that some extra care is needed, because $\rho(c)$ and $\bar{\rho}(c)$ are, just as $z(c)$ and $\bar{z}(c)$, not globally holomorphic and are defined only up to permutation (except in $d=2$, see below), and because (6.11) contains in general non-integer powers.

To begin with, we rewrite eq. (6.11) equivalently as

$$
g\left(c_{E}\right)=\sum_{\delta, 0 \leqslant m \leqslant \delta}(\rho \bar{\rho})^{\delta / 2-m / 2}\left(p_{\delta, m} \rho^{m}+p_{\delta,-m} \bar{\rho}^{m}\right),
$$

Various pieces of this formula need to be analytically continued to the forward tube. Consider first

$$
R(c)=\rho(c) \bar{\rho}(c),
$$

which is a candidate for the analytic continuation of $\rho \bar{\rho}$ from the Euclidean region. We can view it as a composition of two functions: $c \mapsto(\rho(c), \bar{\rho}(c))$ which is a continuous function from $\mathcal{T}_{4}$ to $\mathbb{C}^{2} / \mathbb{Z}_{2}$ analytic away from $\Gamma$, followed by $(\rho, \bar{\rho}) \mapsto \rho \bar{\rho}$ which is a continuous holomorphic function from $\mathbb{C}^{2} / \mathbb{Z}_{2}$ to $\mathbb{C}$. Hence $R(c)$ is a continuous function on the forward tube, analytic everywhere except perhaps on $\Gamma$. However, manifold $\Gamma$ has complex codimension one, and by an analogue of Riemann's theorem about removable singularities we conclude that $R(c)$ is in fact analytic also on $\Gamma$, and thus on the whole $\mathcal{T}_{4} \cdot{ }^{47}$

In addition, $R(c)$ is nonzero in the forward tube. Thus we can lift $R(c)$ to a holomorphic function $\tilde{R}(c)$ from the forward tube to the universal cover $\widetilde{\mathbb{C} \backslash\{0\}}$. Composing this function with $z^{h}: \widetilde{\mathbb{C} \backslash\{0\}} \rightarrow \mathbb{C}$, we obtain an analytic continuation of $(\rho \bar{\rho})^{h}$ for any $h \in \mathbb{R}$, which we denote by $R_{h}(c)$. This discussion mirrors the one around eq. (5.4) in section 5. However, unlike $x_{i j}^{2}$ in that discussion, it is not true that $\tilde{R}(c)$ always belongs to the principal sheet of $\widetilde{\mathbb{C} \backslash\{0\}}$. So, in general, to compute the phase of the analytically continued function, one should follow the phase of $\rho \bar{\rho}$ along a curve joining $c_{E}$ to $c$.

Following a curve is perfectly fine as a theoretical device. For practical computations of the phase, one may wish to use instead the following trick which avoids having to look at the curve. (The reader happy to follow the curve may skip the trick and go directly to eq. (6.26).) Consider the identity:

$$
\rho \bar{\rho}=\frac{1}{16} u(1+\rho)^{2}(1+\bar{\rho})^{2}=\frac{1}{16} \frac{x_{12}^{2} x_{34}^{2}}{x_{13}^{2} x_{24}^{2}} Y^{2}, \quad Y=(1+\rho)(1+\bar{\rho}),
$$

which follows by using $z=\frac{4 \rho}{(1+\rho)^{2}}$, the inverse of the relation (6.9) between $\rho$ and $z$, as well as $u=z \bar{z}$ and the expression for $u$. The function $Y(c)=(1+\rho(c))(1+\bar{\rho}(c))$ is holomorphic on $\mathcal{T}_{4}$ by the same "analyticity on $\mathcal{T}_{4} \backslash \Gamma$ plus Riemann's theorem" argument as used above

\footnotetext{
${ }^{47}$ The precise argument is as follows. Let us keep all complex coordinates fixed and vary just one, say $x_{1}^{0}$. There are two cases: either (6.19) is identically zero as a function of $x_{1}^{0}$, or it is a nonzero polynomial of $x_{1}^{0}$. In the first case $R(c)$ is trivially holomorphic in $x_{1}^{0}$. In the second case (6.19) vanishes at most for a few isolated values of $x_{1}^{0}$. We can then apply 1d Riemann's theorem to say that $R(c)$ is also analytic at those isolated points. By these arguments, we conclude that $R(c)$ is holomorphic in each variable separately. Finally, a continuous function of several complex variables holomorphic in each variable separately is jointly holomorphic [68].
} 
for $\rho \bar{\rho}$. In addition, and this is the key point, because $|\rho(c)|,|\bar{\rho}(c)|<1$, we know that $Y(c) \in \mathbb{C} \backslash(-\infty, 0]$. The upshot of the trick is that eq. (6.24) expresses $\rho \bar{\rho}$ as a product of factors which all remain on the principal sheet of $z^{h}$ upon the analytic continuation. Hence we can compute the analytic continuation of $(\rho \bar{\rho})^{h}$ by

$$
R_{h}(c)=\frac{1}{16^{h}} \frac{\left(x_{12}^{2}\right)^{h}\left(x_{34}^{2}\right)^{h}}{\left(x_{13}^{2}\right)^{h}\left(x_{24}^{2}\right)^{h}} Y(c)^{2 h} \quad(h \in \mathbb{R}),
$$

This determines the phase of $R_{h}(c)$ unambiguously without having to look at the curve joining $c_{E}$ to $c$.

Next, we consider for an integer $m$ a function

$$
\Phi_{m}(c)=\rho(c)^{m}+\bar{\rho}(c)^{m} .
$$

Just as $\rho \bar{\rho}$ and $Y$, it is continuous on $\mathcal{T}_{4}$ and holomorphic on $\mathcal{T}_{4} \backslash \Gamma$, and thus holomorphic on the whole $\mathcal{T}_{4}$.

We can now define the analytic continuation of (6.22). Consider first $d \geqslant 3$, when $p_{\delta,-m}=p_{\delta, m}$. In this case the analytic continuation is given by the formula

$$
g(c)=\sum_{m, \delta, 0 \leqslant m \leqslant \delta} p_{\delta, m} R_{\delta / 2-m / 2}(c) \Phi_{m}(c)
$$

This series consists of holomorphic functions, and it reduces to (6.22) in the Euclidean region. Furthermore, every term in the series can be bounded in absolute value by:

$$
\begin{aligned}
\left|p_{\delta, m} R^{\delta / 2-m / 2}(c) \Phi_{m}(c)\right| & \leqslant p_{\delta, m}|\rho(c) \bar{\rho}(c)|^{\delta / 2-m / 2}\left(|\rho(c)|^{m}+|\bar{\rho}(c)|^{m}\right) \\
& \leqslant p_{\delta, m} r^{\delta-m}\left(r^{m}+r^{m}\right),
\end{aligned}
$$

where $r=r(c)=\max (|\rho(c)|,|\bar{\rho}(c)|)$, which is $<1$ by eq. (6.21). Here we used $p_{\delta, m} \geqslant 0$ in the first line, and $\delta-m \geqslant 0$ in the second line. The terms in the r.h.s. of (6.28) comprise a positive convergent series whose sum is the Euclidean 4-point function (6.22) evaluated at $\rho=\bar{\rho}=r(c)$. This proves that (6.27) converges uniformly on compact subsets of $\mathcal{T}_{4}$, and hence defines a holomorphic function in $\mathcal{T}_{4}$.

It remains to consider $d=2$. As anticipated above, in this case the functions $z(c), \bar{z}(c)$ are individually globally holomorphic on $\mathcal{T}_{4}$. This can be seen introducing coordinates (see [37], section 3.5)

$$
z_{k}=x_{k}^{0}+i x_{k}^{1}, \quad \bar{z}_{k}=x_{k}^{0}-i x_{k}^{1}, \quad k=1,2,3,4 .
$$

Then the explicit formulas for $z(c), \bar{z}(c)$ are given by:

$$
z(c)=\frac{\left(z_{1}-z_{2}\right)\left(z_{3}-z_{4}\right)}{\left(z_{1}-z_{3}\right)\left(z_{2}-z_{4}\right)}, \quad \bar{z}(c)=\frac{\left(\bar{z}_{1}-\bar{z}_{2}\right)\left(\bar{z}_{3}-\bar{z}_{4}\right)}{\left(\bar{z}_{1}-z_{3}\right)\left(\bar{z}_{2}-\bar{z}_{4}\right)} .
$$

The functions $\rho(c), \bar{\rho}(c)$ defined by (6.20) are also individually globally holomorphic on $\mathcal{T}_{4}$. As a consequence, the functions $\rho(c)^{m}$ and $\bar{\rho}(c)^{m}$ are individually holomorphic in $d=2$, 
and not just their sum (6.26). We can therefore define the analytic continuation of $g(c)$ by the formula (compare (6.27)):

$$
g(c)=\sum_{m, \delta, 0 \leqslant m \leqslant \delta} R_{\delta / 2-m / 2}(c)\left[p_{\delta, m} \rho(c)^{m}+p_{\delta,-m} \bar{\rho}(c)^{m}\right] .
$$

This formula would be appropriate for non-parity invariant 2d CFTs which may have $p_{\delta, m} \neq p_{\delta,-m}$. Analyticity follows from the uniform convergence on compact subsets, by the same argument as for $d \geqslant 3$.

Finally, we wish to explain how the above construction may be translated into the language of section 6.1, to see how the issues raised there are resolved. This is instructive but not strictly speaking necessary, so we will be schematic. In the $2 \mathrm{~d}$ case, when $\rho(c)$, $\bar{\rho}(c)$ are individually defined, the translation is in terms of the map

$$
\Omega: c \mapsto(\rho(c), \bar{\rho}(c)) \in(\widetilde{\mathbb{D} \backslash\{0\}})^{2},
$$

where $\mathbb{D}$ is the open unit disk, and we lifted each of the maps $\rho(c), \overline{\rho(c)}$ to the universal cover of $\mathbb{D} \backslash\{0\}$. This map is the present analogue of $\omega$ in (6.3). The function $g(\rho, \bar{\rho})$ extends analytically to the whole $(\widehat{\mathbb{D} \backslash\{0\}})^{2}$, which makes it unnecessary to understand the precise shape of $\Omega\left(\mathcal{T}_{4}\right)$.

For $d \geqslant 3, \rho(c), \bar{\rho}(c)$ are defined only up to permutation. Translation can then be done in terms of their symmetric combinations $\rho \bar{\rho}, \rho+\bar{\rho}$. Any symmetric polynomial in $\rho, \bar{\rho}$, such as the r.h.s. of (6.26), can be expressed as a polynomial in these coordinates. Let then $X$ be the image of $(\mathbb{D} \backslash\{0\})^{2}$ under the map $(\rho, \bar{\rho}) \mapsto(\rho \bar{\rho}, \rho+\bar{\rho})$. The following map is holomorphic on $\mathcal{T}_{4}$ :

$$
\Omega: c \mapsto(\rho(c) \bar{\rho}(c), \rho(c)+\bar{\rho}(c)) \in \tilde{X},
$$

where we lifted to the universal cover. The above argument can be interpreted as showing that the function $g(\rho, \bar{\rho})$ extends analytically to the whole $\tilde{X}$. Understanding the precise shape of $\Omega\left(\mathcal{T}_{4}\right)$ is once again unnecessary.

\subsection{Proof of $z, \bar{z} \notin[1,+\infty)$}

Here we will prove lemma 6.1 which played such a fundamental role in the previous section. Just as for lemma 5.1, it will be helpful to use the Minkowski metric. Thus we pass from Euclidean complex coordinates $x_{k} \in \mathbb{C}^{d}$ to Minkowski complex coordinates $\zeta_{k}=\left(i x_{k}^{0}, \mathbf{x}_{k}\right) \in$ $\mathbb{C}^{1, d-1}$. Definitions of $u, v$ are then rewritten equivalently as

$$
u=\frac{\zeta_{12}^{2} \zeta_{34}^{2}}{\zeta_{13}^{2} \zeta_{24}^{2}}, \quad v=\frac{\zeta_{23}^{2} \zeta_{14}^{2}}{\zeta_{13}^{2} \zeta_{24}^{2}}
$$

where $\zeta_{i j}=\zeta_{i}-\zeta_{j}$ and $\zeta^{2}=-\left(\zeta^{0}\right)^{2}+\zeta^{2}$. We denote

$$
\zeta_{k}=\xi_{k}+i \eta_{k}, \quad \xi_{k}, \eta_{k} \in \mathbb{R}^{1, d-1} .
$$

We will thus use Minkowski norm for $\xi$ 's, $\eta$ 's and their differences. The forward tube condition on $x_{k}$ is rewritten as $\eta_{k}-\eta_{k+1} \succ 0$ which is the notation for

$$
\eta_{k}^{0}-\eta_{k+1}^{0}>0 \text { and } \quad-\left(\eta_{k}-\eta_{k+1}\right)^{2}>0 .
$$


We will need the following lemma which is related to lemma 5.1 (see the proof at the end of the section).

Lemma 6.2. Let $\zeta=\xi+i \eta$ and $\eta^{2}<0$. Then

(a) $\zeta^{2} \neq 0$;

(b) Define $\zeta^{\prime}=\xi^{\prime}+i \eta^{\prime}$ by

$$
\zeta^{\prime}=\zeta / \zeta^{2}
$$

which is finite by Part (a). Then $\eta^{\prime}$ belongs to the same causal part of the light cone (future or past) as $\eta$. I.e. $\eta \succ 0 \Rightarrow \eta^{\prime} \succ 0$. Analogously, $\eta \prec 0 \Rightarrow \eta^{\prime} \prec 0$.

Let us start the proof of lemma 6.1. The $z, \bar{z}$ are defined from $u, v$ via (6.6). It is not hard to see from the first line of (6.6) that $z, \bar{z}$ are precisely the two solutions of the quadratic equation

$$
z^{2}-(1+u-v) z+u=0
$$

We thus have to show that, assuming (6.36), this equation has no solutions which are real and belong to the interval $[1,+\infty)$.

Without loss of generality, we can assume that $\zeta_{3}=0 .{ }^{48}$ Then we have $\eta_{1}, \eta_{2} \succ 0$ while $\eta_{4} \prec 0$. Then we apply lemma 6.2 and map the configuration $\left(\zeta_{1}, \zeta_{2}, 0, \zeta_{4}\right)$ to the configuration $\left(\zeta_{1}^{\prime}, \zeta_{2}^{\prime}, \infty, \zeta_{4}^{\prime}\right)$ with $\eta_{1}^{\prime}, \eta_{2}^{\prime} \succ 0$ while $\eta_{4}^{\prime} \prec 0$. These relations imply $\eta_{14}^{\prime} \succ$ $0, \eta_{24}^{\prime} \succ 0$ which will be used below.

Since $u, v$ are invariant under the inversion, we have (this can be checked by a direct computation)

$$
u=\frac{\left(\zeta_{12}^{\prime}\right)^{2}}{\left(\zeta_{24}^{\prime}\right)^{2}}, \quad v=\frac{\left(\zeta_{14}^{\prime}\right)^{2}}{\left(\zeta_{24}^{\prime}\right)^{2}}
$$

and eq. (6.38) reduces to

$$
\left(\zeta_{24}^{\prime}\right)^{2} z^{2}-\left[\left(\zeta_{24}^{\prime}\right)^{2}+\left(\zeta_{12}^{\prime}\right)^{2}-\left(\zeta_{14}^{\prime}\right)^{2}\right] z+\left(\zeta_{12}^{\prime}\right)^{2}=0
$$

Using that $\zeta_{12}^{\prime}=\zeta_{14}^{\prime}-\zeta_{24}^{\prime}$, this equation can be written equivalently as

$$
\left(\zeta_{14}^{\prime}+(z-1) \zeta_{24}^{\prime}\right)^{2}=0
$$

Now let us suppose that $z \in[1,+\infty)$. Then

$$
\operatorname{Im}\left[\zeta_{14}^{\prime}+(z-1) \zeta_{24}^{\prime}\right]=\eta_{14}^{\prime}+(z-1) \eta_{24}^{\prime} \succ 0 .
$$

Then eq. (6.41) is in contradiction with lemma 5.1. lemma 6.1 is demonstrated.

\footnotetext{
${ }^{48} \mathrm{It}$ is important to move $\zeta_{3}$ (or $\zeta_{2}$ ) to zero rather than $\zeta_{1}$ or $\zeta_{4}$, because only then, after applying the inversion, one gets causal information not only on $\eta_{k}^{\prime}$ 's but also on some of their differences.
} 
Proof of lemma 6.2. This was shown in [55], footnote 74, and we reproduce the argument here for completeness. Part (a) is a partial case of lemma 5.1 (for $\eta \prec 0$ we should apply it to the complex conjugate vector $\left.\zeta^{*}=\xi-i \eta\right)$. Let us show Part (b). To show that $\eta \succ 0 \Rightarrow \eta^{\prime} \succ 0$, we write

$$
\zeta^{\prime}=\frac{\xi+i \eta}{\xi^{2}-\eta^{2}+2 i(\xi, \eta)}=\frac{(\xi+i \eta)\left(\xi^{2}-\eta^{2}-2 i(\xi, \eta)\right)}{\left(\xi^{2}-\eta^{2}\right)^{2}+4(\xi, \eta)^{2}} .
$$

So, up to a positive factor, $\eta^{\prime}$ is given by

$$
\left(\xi^{2}-\eta^{2}\right) \eta-2(\xi, \eta) \xi
$$

For $\xi=0$ this is given by $\left(-\eta^{2}\right) \eta \succ 0$. More generally, this squares to

$$
\left(\xi^{2}-\eta^{2}\right)^{2} \eta^{2}+4(\xi, \eta)^{2} \xi^{2}-4(\xi, \eta)^{2}\left(\xi^{2}-\eta^{2}\right)=\eta^{2}\left(\left(\xi^{2}-\eta^{2}\right)^{2}+4(\xi, \eta)^{2}\right)<0 .
$$

Therefore, for all $\xi$, we have that $\eta^{\prime}$ is timelike. Since we have shown that $\eta^{\prime} \succ 0$ for $\xi=0$, by continuity it follows that $\eta^{\prime} \succ 0$ for all $\xi$.

Finally, the implication $\eta \prec 0 \Rightarrow \eta^{\prime} \prec 0$ follows by complex conjugation.

\subsection{4-point function powerlaw bound}

We wish to show next that the analytically continued 4-point function satisfies a powerlaw bound, so that we can apply theorem 4.1. The prefactor in eq. (6.17) satisfies a powerlaw bound by lemma 5.2. Furthermore, eq. (6.28) implies that the analytic continuation $g(c)$ constructed in section 6.3 is bounded by a Euclidean 4-point function, namely:

$$
|g(c)| \leqslant g_{E}\left(c_{*}\right)
$$

where $c_{*}$ is any Euclidean 4-point function configuration having $\rho\left(c_{*}\right)=\bar{\rho}\left(c_{*}\right)=r=r(c)=$ $\max (|\rho(c)|,|\bar{\rho}(c)|)$. We choose the conformal frame (6.10):

$$
c_{*}: \quad x_{1}=-r \widehat{e}_{0}, x_{2}=r \widehat{e}_{0}, x_{3}=\widehat{e}_{0}, x_{4}=-\widehat{e}_{0} .
$$

Using the convergent OPE in the $x_{2} \rightarrow x_{3}, x_{1} \rightarrow x_{4}$ channel, we have the asymptotics

$$
G_{4}^{E}\left(c_{*}\right) \sim \frac{1}{(1-r)^{4 \Delta_{\varphi}}} \quad(r \rightarrow 1) .
$$

The function $g_{E}\left(c_{*}\right)$ satisfies the same asymptotics up to a constant, being related to $G_{4}^{E}\left(c_{*}\right)$ via eq. (6.1) by a factor which is non-singular in the $r \rightarrow 1$ limit. Since $g_{E}\left(c_{*}\right)$ is a positive monotonically increasing function for $0 \leqslant r<1$ (see eq. (6.11)), we conclude that it has a bound

$$
g_{E}\left(c_{*}\right) \leqslant \frac{\text { const. }}{(1-r(c))^{4 \Delta_{\varphi}}}
$$

and $|g(c)|$ by (6.46) satisfies the same bound.

The upshot of this discussion is that we will have a powerlaw bound on $G_{4}(c)$ if we manage to get a powerlaw bound on $\frac{1}{1-r(c)}$. We will next state and prove such a bound. 
Before launching into the technical discussion, let us discuss intuitively why a result like this is expected to be true. We know (lemma 6.1) that $|\rho(c)|,|\bar{\rho}(c)|<1$ and now we wish to prove that $|\rho(c)|,|\bar{\rho}(c)|$ do not approach 1 too quickly as $c$ goes to the Minkowski boundary of the forward tube. This may remind the reader of the Schwarz-Pick lemma, which says that if $f(w)$ is a function from a unit disk to itself and $f(0)=0$, then $|f(w)| \leqslant$ $|w|$, hence providing a bound on how fast $|f(w)|$ can approach 1 as $|w| \rightarrow 1$. In the $2 \mathrm{~d}$ case, when $\rho(c)$ and $\bar{\rho}(c)$ are individually defined holomorphic functions in the forward tube, it is indeed possible to use the Schwarz-Pick lemma to prove a powerlaw bound on $\max (|\rho(c)|,|\bar{\rho}(c)|)[69]$. It should be possible to generalize the Schwarz-Pick argument to any $d$, although we have not worked it out in full details. ${ }^{49}$ The proof below will be different and more direct: it will simply mimic the proof of lemma 6.1 , replacing all " $>0$ " inequalities by " $\geqslant \varepsilon$ " with an explicit positive $\varepsilon$.

\subsubsection{A powerlaw bound on $\frac{1}{1-r(c)}$}

Let us introduce some notation. We will measure the size of a complex vector $\zeta \in \mathbb{C}^{1, d-1}$ by $|\zeta|$,

$$
|\zeta|^{2}=\left|\zeta^{0}\right|^{2}+\left|\zeta^{1}\right|^{2}+\cdots+\left|\zeta^{d-1}\right|^{2}
$$

Clearly $\left|\left(\zeta_{1}, \zeta_{2}\right)\right| \leqslant\left|\zeta_{1}\right|\left|\zeta_{2}\right|$. We also define for $\zeta=\xi+i \eta, \xi, \eta \in \mathbb{R}^{1, d-1}$, and $\eta^{2}<0$ (i.e. timelike)

$$
S(\zeta)=\max \left(\frac{1}{\sqrt{-\eta^{2}}},|\zeta|\right)
$$

Thus $S(\zeta)$ is large either if some component of $\zeta$ (real or imaginary) is large or if $\eta$ approaches the light cone. Note that $S(\zeta) \geqslant 1$ for any $\zeta$. We will never need $S(\xi+i \eta)$ for spacelike $\eta$.

Finally we consider an analogous function on $\mathcal{T}_{4}$ :

$$
S(c)=\max _{i<j} S\left(\zeta_{i j}\right),
$$

which becomes large if any of $S\left(\zeta_{i j}\right)$ become large. We claim that there is the following bound $($ recall $r(c)=\max (|\rho(c)|,|\bar{\rho}(c)|))$

$$
\frac{1}{1-r(c)} \leqslant 720 S(c)^{12} \quad\left(c \in \mathcal{T}_{4}\right)
$$

This bound will be shown for any $c$ in the forward tube, which is the natural setting. When we specify to $c \in \mathcal{D}_{4} \subset \mathcal{T}_{4}$ [see eq. (4.5)], we have

$$
S(c)=\max _{i<j} \max \left\{\frac{1}{\left|\epsilon_{i}-\epsilon_{j}\right|},\left|x_{i}-x_{j}\right|\right\} .
$$

Eq. (6.53) then becomes a powerlaw bound for $\frac{1}{1-r(c)}$ on $\mathcal{D}_{4}$ of the form (4.9), precisely as needed for applying theorem 4.1 .

\footnotetext{
${ }^{49}$ For any $d$, the Schwarz-Pick lemma allows a natural generalization to holomorphic functions in the forward tube [70].
} 
The proof of the bound (6.53) will build upon the proof of $z, \bar{z} \notin[1,+\infty)$ given in section 6.4. There we showed that $z$ solves eq. (6.41), which however is inconsistent for $z \in[1,+\infty)$ and $c$ in the forward tube. Here we will use the same eq. (6.41), but make the rest of the argument quantitative, by showing that if $c$ stays away from the boundary or infinity of the forward tube, so that $S(c)$ is bounded, then both $z(c)$ and $\bar{z}(c)$ must stay a finite distance away from $[1,+\infty)$, as measured by an upper bound on $\frac{1}{1-r(c)}$ expressed by eq. (6.53). The proof is straightforward but somewhat technical and we split it into a series of lemmas.

Lemma 6.3. Let $\zeta=\xi+i \eta, \eta^{2}<0$. Then for any $\xi$

$$
\left|\zeta^{2}\right| \geqslant\left(-\eta^{2}\right)
$$

Proof. This is a generalization of lemma 5.2(a) and could be proven analogously. We give a slightly different proof for a change. We have

$$
\left|\zeta^{2}\right|^{2}=\left(\xi^{2}-\eta^{2}\right)^{2}+4(\xi, \eta)^{2}=\left(\xi^{2}\right)^{2}+\left(\eta^{2}\right)^{2}+2\left[2(\xi, \eta)^{2}-\xi^{2} \eta^{2}\right] .
$$

The lemma now follows from the inequality:

$$
2(\xi, \eta)^{2}-\xi^{2} \eta^{2} \geqslant 0
$$

Eq. (6.57) is obvious for $\xi^{2} \geqslant 0$, so let us consider $\xi^{2}<0$. By Lorentz invariance and homogeneity it's enough to consider $\xi=( \pm 1,0, \ldots, 0)$ in which case the l.h.s. of (6.57) becomes $\left(\eta^{0}\right)^{2}+\boldsymbol{\eta}^{2} \geqslant 0$.

Then we have the following strengthening of lemma $6.2(\mathrm{~b})$ :

Lemma 6.4. Let $\zeta=\xi+i \eta, \eta^{2}<0$, and $\zeta^{\prime}=\zeta / \zeta^{2}$. Then

$$
S\left(\zeta^{\prime}\right) \leqslant[S(\zeta)]^{3}
$$

Proof. We have

$$
\left|\zeta^{\prime}\right|=\frac{|\zeta|}{\left|\zeta^{2}\right|} \leqslant[\text { by lemma } 6.3] \frac{|\zeta|}{-\eta^{2}} \leqslant S(\zeta)^{3}
$$

We also have (see the proof of lemma 6.2, in particular eq. (6.45)) that $\eta^{\prime 2}<0$ and

$$
\frac{1}{-\eta^{\prime 2}}=\frac{\left|\zeta^{2}\right|^{2}}{-\eta^{2}} \leqslant[\text { by lemma } 6.3] S(\zeta)^{6} \text {. }
$$

Lemma 6.5. Let $\zeta_{i} \in \mathbb{C}^{1, d-1}, \eta_{i} \succ 0(i=1,2)$. Then

$$
S\left(\zeta_{1}+\zeta_{2}\right) \leqslant S\left(\zeta_{1}\right)+S\left(\zeta_{2}\right)
$$

Proof. We have $\left|\zeta_{1}+\zeta_{2}\right| \leqslant\left|\zeta_{1}\right|+\left|\zeta_{2}\right|$ and $-\left(\eta_{1}+\eta_{2}\right)^{2} \geqslant-\eta_{1}^{2}-\eta_{2}^{2}\left(\right.$ since $\left.\eta_{1} \cdot \eta_{2}<0\right)$. 
Lemma 6.6. Let $\Upsilon_{i}=\Phi_{i}+i \Psi_{i} \in \mathbb{C}^{1, d-1}, \Phi_{i}, \Psi_{i} \in \mathbb{R}^{1, d-1}, \Psi_{i} \succ 0(i=1,2)$, and $z$ solves the equation

$$
\left(\Upsilon_{1}+(z-1) \Upsilon_{2}\right)^{2}=0
$$

Then

$$
1-|\rho(z)| \geqslant \delta_{0}:=\frac{1}{45 S^{4}}, \quad S=\max \left(S\left(\Upsilon_{1}\right), S\left(\Upsilon_{2}\right)\right)
$$

Proof. Note that $z=4 \rho /(1+\rho)^{2}$, and so eq. (6.61) can be rewritten as

$$
\left((\rho+1)^{2} \Upsilon_{1}-(\rho-1)^{2} \Upsilon_{2}\right)^{2}=0
$$

For $\rho=e^{i \alpha}$, multiplying this equation by $e^{-2 i \alpha}$, it becomes

$$
(\Upsilon)^{2}=0, \quad \Upsilon \equiv\left(2 \cos \frac{\alpha}{2}\right)^{2} \Upsilon_{1}+\left(2 \sin \frac{\alpha}{2}\right)^{2} \Upsilon_{2}
$$

which contradicts lemma 6.2(a), since $\operatorname{Im} \Upsilon \succ 0$. So $\rho$ cannot lie precisely on the unit circle (as we already knew). It should then not be surprising that it also cannot get too close to the unit circle, which is what (6.62) says. This can be shown by a straightforward although somewhat technical generalization of the above argument.

Denoting $\rho=r e^{i \alpha}=e^{i \alpha}-\delta e^{i \alpha}, \delta=1-r>0$, and multiplying (6.63) by $e^{-2 i \alpha}$, it becomes

$$
\left(\left(2 \cos \frac{\alpha}{2}-\delta e^{i \alpha / 2}\right)^{2} \Upsilon_{1}+\left(2 \sin \frac{\alpha}{2}+i \delta e^{i \alpha / 2}\right)^{2} \Upsilon_{2}\right)^{2}=0
$$

or

$$
\left(\Upsilon+\Upsilon^{\prime}\right)^{2}=0
$$

with

$$
\begin{aligned}
\Upsilon & =4 \cos ^{2} \frac{\alpha}{2} \Upsilon_{1}+4 \sin ^{2} \frac{\alpha}{2} \Upsilon_{2}, \\
\Upsilon^{\prime} & =\kappa_{1} \Upsilon_{1}+\kappa_{2} \Upsilon_{2}, \\
\kappa_{1} & =-4 \cos \frac{\alpha}{2} \delta e^{i \alpha / 2}+\delta^{2} e^{i \alpha}, \quad \kappa_{2}=4 i \sin \frac{\alpha}{2} \delta e^{i \alpha / 2}-\delta^{2} e^{i \alpha} .
\end{aligned}
$$

So for $\delta$ small, $\Upsilon^{\prime}$ is a small correction to $\Upsilon$. We write $\operatorname{Im}\left(\Upsilon+\Upsilon^{\prime}\right)=\Psi+\Psi^{\prime}$, where

$$
\Psi=\operatorname{Im} \Upsilon=4 \cos ^{2} \frac{\alpha}{2} \Psi_{1}+4 \sin ^{2} \frac{\alpha}{2} \Psi_{2}, \quad \Psi^{\prime}=\operatorname{Im} \Upsilon^{\prime} .
$$

We know that $\Psi \succ 0$. In addition we also have a lower bound on $-\Psi^{2}$ :

$$
-\Psi^{2} \geqslant 16 \cos ^{4} \frac{\alpha}{2}\left(-\Psi_{1}^{2}\right)+16 \sin ^{4} \frac{\alpha}{2}\left(-\Psi_{2}^{2}\right) \geqslant \frac{1}{S^{2}} \times 16 \min \left\{\cos ^{4} \frac{\alpha}{2}, \sin ^{4} \frac{\alpha}{2}\right\}=\frac{4}{S^{2}} .
$$


We will now show that $-\left(\Psi+\Psi^{\prime}\right)^{2}$ remains strictly positive if $\delta<\delta_{0}$. This will imply, by lemma 6.2(a), that eq. (6.66) cannot hold, and hence we must have $\delta \geqslant \delta_{0}$, i.e. eq. (6.62), proving the lemma.

To implement this natural strategy, we will need only crude estimates of the size of various terms. Note that $\delta_{0}<1$ since $S \geqslant 1$, so in particular we have $\delta^{2} \leqslant \delta$. Using this we have the bounds $\left|\kappa_{i}\right| \leqslant 5 \delta$, and hence an upper bound

$$
\left|\Psi^{\prime}\right| \leqslant\left|\Upsilon^{\prime}\right| \leqslant 10 \delta S
$$

We also have an upper bound $|\Psi| \leqslant 4 S$. Using these, (6.70), and $\delta^{2} \leqslant \delta$, we have:

$$
\begin{aligned}
-\left(\Psi+\Psi^{\prime}\right)^{2}=-\Psi^{2}-2\left(\Psi, \Psi^{\prime}\right)-\left(\Psi^{\prime}\right)^{2} & \geqslant \frac{4}{S^{2}}-2|\Psi|\left|\Psi^{\prime}\right|-\left|\Psi^{\prime}\right|^{2} \\
& \geqslant \frac{4}{S^{2}}-80 \delta S^{2}-100 \delta^{2} S^{2} \\
& \geqslant \frac{4}{S^{2}}-180 \delta S^{2}=\frac{4\left(1-\delta / \delta_{0}\right)}{S^{2}},
\end{aligned}
$$

which is strictly positive for $\delta<\delta_{0}$. As explained above this proves the lemma.

Finally we can prove (6.53). We repeat the proof of lemma 6.1 given in section 6.4. As there, we reduce to configuration having $\zeta_{3}=0$ and obtain that $z$ (as well as $\bar{z}$ ) is a solution of eq. (6.41), which has the form (6.61) with

$$
\Upsilon_{1}=\zeta_{14}^{\prime}=\zeta_{1}^{\prime}-\zeta_{4}^{\prime}, \quad \Upsilon_{2}=\zeta_{24}^{\prime}=\zeta_{2}^{\prime}-\zeta_{4}^{\prime}, \quad \zeta_{i}^{\prime}=\zeta_{i} / \zeta^{2} \quad(i=1,2,4)
$$

Let us write $\Upsilon_{i}=\Phi_{i}+i \Psi_{i} \in \mathbb{C}^{1, d-1}, \Phi_{i}, \Psi_{i} \in \mathbb{R}^{1, d-1}$. As was already pointed out in section 6.4 , we have $\Psi_{i} \succ 0(i=1,2)$. Furthermore, by lemma 6.4 we know that $S\left(\zeta_{i}^{\prime}\right) \leqslant$ $S(c)^{3}$, and then applying lemma 6.5 that $S\left(\Upsilon_{i}\right) \leqslant 2 S(c)^{3}$. Thus lemma 6.6 implies (6.53) (note that $720=45 \times 16$ ).

Remark 6.1. The bound (6.53) is not optimal. We will prove a better bound in section 7 , by a different argument.

Let us recap. In section 6.3 we have analytically continued the Euclidean 4-point function to the forward tube, and here we showed that this analytic continuation satisfies a powerlaw bound. Then by theorem 4.1, the Minkowski 4-point function defined as the limit (4.8) exists, is a Lorentz-invariant tempered distribution, and satisfies Wightman spectral condition. In the remainder of this section we will show that this distribution is also conformally invariant (section 6.6), that it satisfies the remaining Wightman axioms (positivity in section 6.7, clustering in section 6.8, and local commutativity in section 6.9). Later in section 8 we will also show that it can be computed by a convergent (in the sense of distributions) OPE.

Now that we know that the Minkowski 4-point function is a distribution everywhere, one may inquire about the regularity of this distribution. E.g. for some configurations the 4-point function is actually real-analytic [37]. We will come back to this question in the conclusion section. 


\subsection{Conformal invariance}

Conformal invariance of Euclidean 4-point function (6.1) can be described as invariance under finite conformal transformations $x \rightarrow x^{\prime}=f(x)$,

$$
\Omega_{1} \Omega_{2} \Omega_{3} \Omega_{4} G_{4}^{E}\left(x_{1}^{\prime}, x_{2}^{\prime}, x_{3}^{\prime}, x_{4}^{\prime}\right)=G_{4}^{E}\left(x_{1}, x_{2}, x_{3}, x_{4}\right),
$$

where $\Omega_{i}=J\left(x_{i}\right)^{\Delta_{\mathcal{O}}}$ and $J(x)=\operatorname{det}\left(\partial f^{\mu} / \partial x^{\nu}\right)^{1 / d}$ is the local scale factor. Alternatively, and equivalently, this can be expressed as invariance under infinitesimal conformal transformations, a conformal Ward identity, which says that the Euclidean correlator is annihilated by a sum of differential operators, one per point:

$$
\sum_{i=1}^{4} \mathcal{D}\left(x_{i}, \partial_{x_{i}}\right) G_{4}^{E}\left(x_{1}, x_{2}, x_{3}, x_{4}\right)=0 .
$$

There is a differential operator per conformal group generator $\left(\partial^{\mu}\right.$ for $P_{\mu}, x^{\mu} \partial^{\nu}-x^{\nu} \partial^{\mu}$ for $M_{\mu \nu}, x \cdot \partial+\Delta_{\mathcal{O}}$ for $D, x^{2} \partial^{\mu}-2 x^{\mu}(x \cdot \partial)-2 x^{\mu} \Delta_{\mathcal{O}}$ for $\left.K_{\mu}\right)$.

Since all these differential operators have polynomial coefficients, Ward identities (6.75) continue to hold in the forward tube for the function $G\left(x_{1}, x_{2}, x_{3}, x_{4}\right)$. Taking the limit to the Minkowski boundary, we obtain that the Minkowski 4-point function satisfies infinitesimal Minkowski conformal invariance expressed by the Ward identities.

The possibility to take the limit is guaranteed by the standard result that distributional limits commute with derivatives. Indeed, suppose that we have, in the sense of distributions, $\lim _{\varepsilon \rightarrow 0} f_{\varepsilon}=g$. This means that for any test function $\varphi$, we have $\lim _{\varepsilon \rightarrow 0}\left(f_{\varepsilon}, \varphi\right)=(g, \varphi)$. But then for any derivative $\partial$,

$$
(\partial g, \varphi)=-(g, \partial \varphi)=-\lim _{\varepsilon \rightarrow 0}\left(f_{\varepsilon}, \partial \varphi\right)=\lim _{\varepsilon \rightarrow 0}\left(\partial f_{\varepsilon}, \varphi\right),
$$

which implies that $\lim _{\varepsilon \rightarrow 0} \partial f_{\varepsilon}=\partial g$. A similar argument shows that the limit commutes with multiplication of distributions by polynomials. All this is analogous to how we prove Lorentz invariance of the Minkowski correlator in appendix C.

So we have shown that the Minkowski 4-point function satisfies Lorentzian conformal Ward identities. This means that

$$
\sum_{i=1}^{4}\left(\mathcal{D}_{i} G_{4}^{M}, \varphi\right)=0,
$$

where $\mathcal{D}_{i}$ are the analytic continuations of the Euclidean differential operators to Minkowski space, and the pairing with the Schwartz test functions is defined by integration by parts. Note that the conformal Ward identities in Minkowski space hold also at coincident points (i.e. the test function $\varphi$ does not have to be zero at coincident points).

Now let us discuss invariance of Minkowski 4-point function under finite Lorentzian conformal transformations. Since $G_{4}^{M}$ is a distribution, the appropriate form of writing is to transform the test function:

$$
\left(G_{4}^{M}, \varphi\right)=\left(G_{4}^{M}, \varphi^{f}\right)
$$




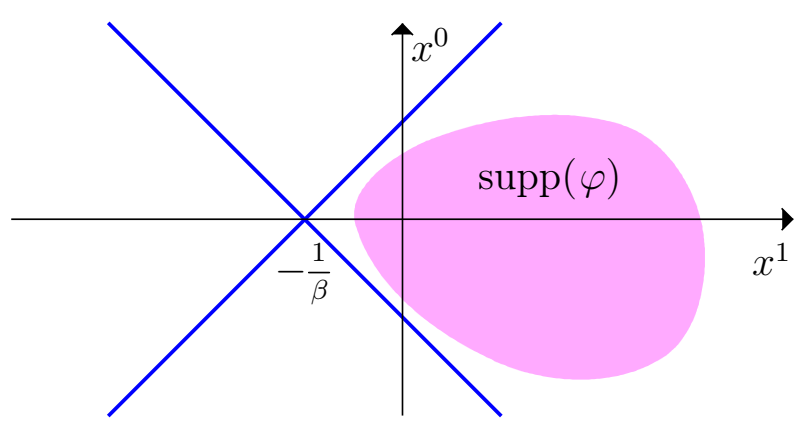

Figure 4. In the $2 \mathrm{~d}$ case, the special conformal transformation (6.80) is singular on the blue light cone $x^{0}= \pm\left|x^{1}+\beta^{-1}\right|$. Suppose $\varphi$ is supported as shown on the right of the light cone. As $\beta \rightarrow 0$, the light cones moves towards the left infinity and does not touch $\operatorname{supp}(\varphi)$. Therefore, such a $\varphi$ satisfies the condition for the invariance under a finite special conformal transformation (6.80).

where $\varphi^{f}\left(x_{1}, \ldots, x_{4}\right)=\varphi\left(f^{-1}\left(x_{1}\right), \ldots, f^{-1}\left(x_{4}\right)\right) \prod_{i=1}^{4} J\left(f^{-1}\left(x_{i}\right)\right)^{\Delta_{\mathcal{O}}-d}$. However we have to be careful. This invariance is true not for every test function $\varphi$ but only for an $f$ dependent subset of test functions.

Let $f_{t}$ be a smooth family of Lorentzian conformal transformations connecting $f$ to the identity: $f_{0}=\mathrm{id}, f_{1}=f$. Suppose that

$$
\varphi^{f_{t}} \text { is a Schwartz function for any } f_{t} \text { in the family. }
$$

Then we can integrate infinitesimal conformal invariance and prove that (6.78) is true. For translations, Lorentz transformations and dilatations, eq. (6.79) is clearly satisfied and eq. (6.78) holds for any $\varphi$. However, for general conformal transformations, (6.79) may not necessarily be true. The problems will appear if $f$ is singular on the support of $\varphi$, as $\varphi^{f}$ may then not be a Schwartz function. As a concrete example, consider the Lorentzian special conformal transformation:

$$
f(x)=\frac{x^{\mu}+x^{2} b^{\mu}}{1+2 x \cdot b+x^{2} b^{2}} .
$$

The corresponding scale factor is $J(x)=\frac{1}{1+2 x \cdot b+x^{2} b^{2}}$. Take for definiteness spacelike $b=\beta \widehat{e}_{1}$, where $\beta>0$ and $\widehat{e}_{1}$ is the unit vector in the $x^{1}$ direction. The transformation (6.80) is then singular for $x^{0}= \pm\left|\mathbf{x}+\beta^{-1} \widehat{e}_{1}\right|$, where the scale factor blows up, i.e. on the light cone whose vertex is at $x=-\beta^{-1} \widehat{e}_{1} .{ }^{50}$ Scaling $\beta$ to zero we can connect the transformation (6.80) to the identity. Under this scaling the light cone of singularities moves away to infinity along the negative $x^{1}$ direction. Requirement (6.79), and hence finite invariance (6.78), will hold if the light cone of singularities, while moving away, does not touch the support of $\varphi$ (see figure 4 for the $2 \mathrm{~d}$ case).

Note that such a support requirement still leaves the possibility for both spacelike and timelike separations among the points $x_{i}$ in the support of $\varphi$. For $x_{i} \in \operatorname{supp} \varphi$, the points $f\left(x_{i}\right)$ will have the same causal structure as the points $x_{i}$, i.e. $\left(f\left(x_{i}\right)-f\left(x_{j}\right)\right)^{2}$ will have the

\footnotetext{
${ }^{50}$ Recall that we are using,$-+\cdots+$ Minkowski signature.
} 
same sign as $\left(x_{i}-x_{j}\right)^{2}$. This follows from the fact that $J(x)>0$ on $\operatorname{supp}(\varphi)$, as guaranteed by being able to continuously connect to the identity without singularities.

In the early CFT days, it was considered puzzling that Lorentzian special conformal transformations may change the causal structure of a point configuration. As we see here, the puzzle can be avoided by either limiting to infinitesimal conformal invariance, or by restricting the class of test functions so that the causal structure is preserved. A third way to deal with the puzzle is to consider the Lorentzian conformal transformations acting on the Lorentzian cylinder as opposed to the Minkowski space [71]. We will revisit the Lorentzian cylinder in our future publication [72].

Remark 6.2. We would like to contrast the Minkowski conformal Ward identities (6.77) with conformal Ward identities valid for Euclidean correlators. Euclidean correlators are real-analytic away from coincident points and naturally satisfy conformal Ward identities for such configurations. Although in this paper we don't need it, in some questions it might be useful to extend Euclidean correlators, in the sense of distributions, also to coincident points. One may ask if such an extension can be done in a way so that the resulting distributional correlators satisfy conformal Ward identities analogous to (6.77). In general the answer is no, already for 2-point functions. Namely 2-point functions of primaries of dimension $\Delta$ such that $2 \Delta-d \in \mathbb{Z}$ will in general not allow even a scale invariant extension at coincident points, let alone conformally invariant one. E.g. this feature will always be present for the stress tensor 2-point function.

\subsubsection{Conformal invariance in terms of cross ratios}

So as we have just seen, Minkowski correlator $G_{4}^{M}$ is conformally invariant. If it were a function, conformal invariance would imply that we could write it as the usual prefactor times a function of the cross-ratios. Since it is a distribution, one might hope that it can be written as the prefactor times a distribution of the cross-ratios. We will now develop this point of view and show that it indeed works, at least locally. ${ }^{51}$

Our goal in this section will be to make sense of the formula:

$$
G_{4}(c)=\frac{g(z(c), \bar{z}(c))}{\left(x_{12}^{2} x_{34}^{2}\right)^{\Delta_{\mathcal{O}}}},
$$

where $g(z, \bar{z})$ will be in general a distribution in two variables, and $g(z(c), \bar{z}(c))$ its pullback to the space $\mathbb{R}^{4 d}$ of Minkowski 4-point configurations $c$. This equation will be understood in the sense of integrating both parts with a test function. Because of the difficulty described in footnote 51 , we will only consider compactly supported $C^{\infty}$ test functions $\varphi(c)$, with the

\footnotetext{
${ }^{51}$ We note right away that one does not expect a very nice global statement. Indeed, the cross-ratio space is morally the moduli space of four points on Minkowski cylinder $\mathcal{M}$. (Note that we have not yet constructed the Wightman functions as distributions on Minkowski cylinder. However, it is not important for the point that we are trying to convey.) This is a quotient space $\left(\mathcal{M}^{4}\right) / G$ where $G$ is the universal cover of Lorentzian conformal group. This quotient space is rather singular, which has to do with different configurations in $\mathcal{M}^{4}$ having different stability subgroups (light-cones, $z=\bar{z}$ ). The quotient space $\left(\mathcal{M}^{4}\right) / G$ is not only not smooth, it is not even Hausdorff. So away from some regular regions of $\left(\mathcal{M}^{4}\right) / G$ one shouldn't expect a simple statement of the form (6.81), unless one finds a smoother model of this moduli space.
} 
additional requirement that all $c \in \operatorname{supp} \varphi$ have the same causal ordering. In particular, this implies that $\operatorname{supp} \varphi$ contains no $c$ 's with lightlike separated pairs. The causal ordering of a configuration $c=\left(x_{1}, x_{2}, x_{3}, x_{4}\right)$ is encoded by the directed graph with vertices $1,2,3,4$ and edges $i \rightarrow j$ if $x_{j}$ belongs to the open future light cone of $x_{i}$ (no edge if two points are spacelike).

Since $u, v$ are real in Minkowski space, $z, \bar{z}$ are either both real (excluding 0,1 ) or complex conjugate. Ref. [37] divided all causal orderings into 4 classes according to possible values of $(z, \bar{z})$ :

- Class S: $(z, \bar{z}) \in(0,1) \times(-\infty, 0)$ or the other way around

- Class T: $(z, \bar{z}) \in(0,1) \times(1,+\infty)$ or the other way around

- Class U: $(z, \bar{z}) \in(-\infty, 0) \times(1,+\infty)$ or the other way around

- Class E causal orderings which contain configurations realizing the remaining possibilities:

$$
\begin{aligned}
& -\mathrm{E}_{\mathrm{su}}:(z, \bar{z}) \in(-\infty, 0) \times(-\infty, 0) \\
& -\mathrm{E}_{\mathrm{st}}:(z, \bar{z}) \in(0,1) \times(0,1) \\
& -\mathrm{E}_{\mathrm{tu}}:(z, \bar{z}) \in(1,+\infty) \times(1,+\infty) \\
& -\mathrm{E}_{\mathrm{stu}}: z, \bar{z} \text { are complex-conjugate and not real }
\end{aligned}
$$

Some class E causal orderings realize only one of the four subclasses, while others contain configurations in each subclass. In the latter case different subclasses are connected along configurations with $z=\bar{z}$ (see figure 2 in [37]).

To simplify the discussion, we will assume that $\operatorname{supp} \varphi$ does not include any configurations with $z=\bar{z}$. In particular, this implies that all configurations from $\operatorname{supp} \varphi$ fall into a single class $\mathrm{S}, \mathrm{T}, \mathrm{U}$ or a single subclass $\mathrm{E}_{\mathrm{su}}, \mathrm{E}_{\mathrm{st}}, \mathrm{E}_{\mathrm{tu}}, \mathrm{E}_{\mathrm{stu}}$. Below we will comment how one can add the $z=\bar{z}$ configurations.

If $\operatorname{supp} \varphi$ falls into class $\mathrm{S}, \mathrm{E}_{\mathrm{su}}, \mathrm{E}_{\mathrm{st}}, \mathrm{E}_{\mathrm{stu}}$, we will have $|\rho|,|\bar{\rho}|<1$. These cases do not require special treatment, since the correlator is a function, and eq. (6.81) is true in the ordinary sense of functions.

If $\operatorname{supp} \varphi$ falls into class $\mathrm{T}$ or $\mathrm{U}$, we will have $|\rho|<1,|\bar{\rho}|=1$ or the other way around. Then $g(z, \bar{z})$ will be a function in $z$ and a distribution in $\bar{z} \cdot{ }^{52}$ This case can be treated analogously, and simpler, than the $|\rho|,|\bar{\rho}|=1$ case discussed below.

Finally, if $\operatorname{supp} \varphi$ falls into class $\mathrm{E}_{\mathrm{tu}}$, we will have $|\rho|,|\bar{\rho}|=1$. Then $g(z, \bar{z})$ will generally be a distribution in two variables. This is the case we will focus on. E.g. it is realized for the causal ordering $1 \rightarrow 3 \rightarrow 2 \rightarrow 4$.

Let us define the distribution $g(z, \bar{z})$ for $z, \bar{z} \in(1,+\infty)$. We first define the distribution $g(\rho, \bar{\rho})$ with $|\rho|,|\bar{\rho}|=1$. This is done using the series in the r.h.s. of eq. (6.22), which we now consider as a function of two independent variables $\rho, \bar{\rho}$. To be precise we consider the series:

$$
g(\rho, \bar{\rho})=\sum_{\delta, 0 \leqslant m \leqslant \delta} e^{i \Phi(\delta / 2-m / 2)}(\rho \bar{\rho})^{\delta / 2-m / 2}\left(p_{\delta, m} \rho^{m}+p_{\delta,-m} \bar{\rho}^{m}\right),
$$

\footnotetext{
${ }^{52}$ For some (but not all) of these causal orderings, it can be shown using another OPE channel that $g(z, \bar{z})$ is actually a function of both variables. See ref. [37].
} 
which we view as a holomorphic function on $(\mathbb{D} \backslash(-1,0])^{2}$. Here $e^{i \Phi}, \Phi \in\{0, \pm 2 \pi, \pm 4 \pi\}$, is the phase acquired by $\rho(c) \bar{\rho}(c)$ upon analytic continuation from Euclidean space (as discussed in section 6.3 this phase is the same as for $u(c))$. This phase is constant for each causal ordering and it may be determined by following a path from $c_{E}$ to $c$ for any particular $c$. Alternatively, the phase can also be determined from (6.24). E.g. the causal ordering $1 \rightarrow 3 \rightarrow 2 \rightarrow 4$ has $\Phi=0 .^{53}$

It's easy to see that function (6.82) satisfies a powerlaw bound as $|\rho|,|\bar{\rho}| \rightarrow 1$. This is a baby version of the problems studied in this paper, which was considered in [36]. The limit of $g\left(r e^{i \theta_{1}}, r e^{i \theta_{2}}\right)$ as $r \rightarrow 1$ defines a tempered distribution on the boundary of the domain of analyticity, parametrized by the two angles $\theta_{1}, \theta_{2}$. We can write it as $g(\rho, \bar{\rho})$, with $\rho, \bar{\rho} \in S^{1}{ }^{54}$

In fact we are interested only in a part of this distribution, because $\rho, \bar{\rho} \neq \pm 1$ for each fixed causal ordering. The points $-1,1$ divide the circle into two open arcs, and within $\operatorname{supp} \varphi, \rho$ and $\bar{\rho}$ will each live in one or the other arc. Each arc is mapped smoothly and one-to-one to $(1,+\infty)$ by the $\rho \mapsto z$ map. Thus we obtain the distribution $g(z, \bar{z})$ defined for $z, \bar{z}>1$. Although in general $z(c), \bar{z}(c)$ are defined only up to permutation, let us define them in the case at hand, with real $z \neq \bar{z}$, so that $\bar{z}(c)>z(c)$.

Now let us go back to making sense of (6.81). Suppose first $g(z(c), \bar{z}(c))$ were a function. Integrating (6.81) against a test function we have:

$$
\int d^{4 d} c G_{4}(c) \varphi(c)=\int d^{4 d} \operatorname{cg}(z(c), \bar{z}(c)) \tilde{\varphi}(c), \quad \tilde{\varphi}(c)=\frac{\varphi(c)}{\left(x_{12}^{2} x_{34}^{2}\right)^{\Delta_{\mathcal{O}}}} .
$$

Note that $\tilde{\varphi}(c)$ is still $C^{\infty}$ since we are away from light cones. We would like to continue by expressing the r.h.s. of the previous equation as an integral of $g(z, \bar{z})$ against a twodimensional test function:

$$
\begin{aligned}
\int d^{4 d} c g(z(c), \bar{z}(c)) \tilde{\varphi}(c) & =\int d z d \bar{z} g(z, \bar{z}) \psi(z, \bar{z}), \\
\psi\left(x_{1}, x_{2}\right) & =\int d^{4} c \delta\left(x_{1}-z(c)\right) \delta\left(x_{2}-\bar{z}(c)\right) \tilde{\varphi}(c) .
\end{aligned}
$$

We would like to know if $\psi\left(x_{1}, x_{2}\right)$ is a smooth function. By our assumptions, $\tilde{\varphi}(c)$ is supported away from $\bar{z}(c)=z(c)$. In this region the map $c \rightarrow(z(c), \bar{z}(c))$ is a submersion, which means that the Jacobian has maximal rank (i.e. 2). Alternatively, this means that the form $d z \wedge d \bar{z}$ does not vanish anywhere away from $z=\bar{z}$. Showing this is a matter of an easy computation. ${ }^{55}$

\footnotetext{
${ }^{53}$ We have that $x_{12}^{2}, x_{34}^{2}, x_{13}^{2}, x_{24}^{2}$ all acquire phase $-\pi$, hence $u=\frac{x_{12}^{2} x_{34}^{2}}{x_{13}^{2} x_{24}^{2}}$ acquires phase 0 .

${ }^{54}$ In [36] we also discussed a more general distribution defined on the product of universal covers of two circles. Here eq. (6.82) with fixed $\alpha$ will be sufficient for our purposes.

${ }^{55}$ Start by noting that, away from $z=\bar{z}$, we have $d z \wedge d \bar{z} \propto d u \wedge d v$ with a nonvanishing prefactor. We need to understand where $\nabla u$ can become proportional to $\nabla v$. Using the embedding space formalism [73] we write $u=\frac{\left(X_{1} X_{2}\right)\left(X_{3} X_{4}\right)}{\left(X_{1} X_{3}\right)\left(X_{2} X_{4}\right)}, v=\frac{\left(X_{1} X_{2}\right)\left(X_{3} X_{4}\right)}{\left(X_{1} X_{3}\right)\left(X_{2} X_{4}\right)}$ where $X_{i}$ are null cone $d+2$ dimensional vectors. For any $X_{i}, X_{j}, X_{k}$ the direction $R_{i, j k}=X_{j}\left(X_{i} X_{k}\right)-X_{k}\left(X_{i}, X_{j}\right)$ is tangent to the null cone at $X_{i}$. Imposing $R_{i, j k} \cdot \nabla_{X_{i}}(u-\alpha v)=0$ for all unequal $i, j, k$ where $\alpha$ is a constant, one gets a set of simple algebraic constraints on $u, v$. These constraints can be easily solved to show that $\alpha=\frac{2 u}{-1+u+v}$ while $(1+u-v)^{2}-4 u=0$. The latter is precisely the constraint characterizing $z=\bar{z}$.
} 
Using the fact that $c \rightarrow(z(c), \bar{z}(c))$ is a submersion, it's easy to show that $\psi\left(x_{1}, x_{2}\right)$ is smooth for $\tilde{\varphi}(c)$ supported away from $\bar{z}(c)=z(c)$ (see Chapter III.1 of [61] for such arguments). To summarize, for every smooth function $\varphi(c)$ compactly supported away from $\bar{z}(c)=z(c)$ and from the light cones, we constructed a smooth function $\psi(z, \bar{z})$ compactly supported in $1<z<\bar{z}$ such that

$$
\int d^{4 d} c G_{4}(c) \varphi(c)=\int d z d \bar{z} g(z, \bar{z}) \psi(z, \bar{z})
$$

holds in case $g(z, \bar{z})$ is a function. We now claim that this equation continues to hold, with the same $\psi$, in case $g(z, \bar{z})$ is a distribution. The point is that we can find a sequence of functions $g_{n}(z, \bar{z})$ which tend to $g(z, \bar{z})$ in the sense of distributions, so that the corresponding $\frac{g_{n}(z(c), \bar{z}(c))}{\left(x_{12}^{2} x_{34}^{2}\right)^{\Delta_{\mathcal{O}}}}$ tend to $G_{4}(c)$ in the sense of distributions on $\mathbb{R}^{4 d}$. Since both $\varphi$ and $\psi$ are smooth, we are allowed to pass to the limit on both sides of the equation, proving the claim. The functions $g_{n}(z, \bar{z})$ are given e.g. by the partial sums of the series (6.82), transformed from the $\rho$ to the $z$ coordinates.

Let us now discuss how configurations where $z=\bar{z}$ can be included into this discussion. The basic difficulty is that the map $c \mapsto(z, \bar{z})$ fails to be a submersion near such configurations. So in general the function $\psi(z, \bar{z})$ will not be smooth. Consider e.g. the causal ordering $1 \rightarrow 3 \rightarrow 2 \rightarrow 4$. In this case it's possible to show (we omit the proof) that the function $\psi(z, \bar{z})$ behaves like

$$
|z-\bar{z}|^{d-2} \text { times a smooth function near } z=\bar{z},
$$

which in general is not smooth unless $d$ is even.

We need to be able to make sense of the r.h.s. of (6.85) for such non-fully-smooth test functions. This is possible due to the following observation. Above we explained, following the arguments first presented in $[36]$, that $g(\rho, \bar{\rho})$ is a distribution for $|\rho|,|\bar{\rho}|=1$. But in fact it's a bit better than that (the fact not mentioned in [36]): it is a distribution in $\rho$ for each fixed value of $\bar{\rho} / \rho=e^{i \alpha}$ ! Indeed if we substitute $\bar{\rho}=e^{i \alpha} \rho$ with a fixed $\alpha$ into (6.82), we get a holomorphic function in the unit disk of $\rho$, which satisfies a powerlaw bound, hence its boundary value is a distribution. This can be generalized to holomorphic maps $\bar{\rho}=f(\rho)$ which maps the unit disk into itself (or at least a portion of the unit disk near $\rho=\rho_{0}$ into the unit disk). Translating to $z, \bar{z}$, this implies in particular that $g(z, z+t)$ is a distribution for any fixed $t$. In fact, the map $\bar{z}=z+t$ corresponds to a map $\bar{\rho}=f_{t}(\rho)$ to which the previous argument is applicable. So $g(z, \bar{z})$ is by no means the most general distribution in two variables, as it allows the restriction to the submanifold $\bar{z}=z+t$ for any $t$. E.g. $\delta(z-\bar{z})$ is not allowed by this property, while $\delta(z+\bar{z})$ is allowed. Following this logic a bit more carefully, it can be shown (we omit the proof) that $g(z, \bar{z})$ can be paired with test functions $\psi(z, \bar{z})$ which, when expressed in terms of $s=z+\bar{z}, t=\bar{z}-z$, have the following property: $\psi(s, t)$ is $C^{\infty}$ with respect to $s$ for any fixed $t$, with bounds on derivatives in the $s$ direction which are integrable in the $t$ direction. Eq. (6.86) is compatible with this requirement.

A further complications arises near the $z=\bar{z}>1$ locus for the causal orderings which include configurations in both $\mathrm{E}_{\mathrm{tu}}$ and $\mathrm{E}_{\mathrm{stu}}$ subclasses. In this case the function $\psi(z, \bar{z})$ 
defined in (6.84) will consist of two functions $\psi_{1}(z, \bar{z})$ and $\psi_{2}(z, \bar{z})$ : one defined for real $z, \bar{z}$, another for complex-conjugate $z, \bar{z}$. The two functions $\psi_{i}$ will be glued along the $z=\bar{z}>1$ line. The resulting glued function will not in generally be smooth on the $z=\bar{z}>1$ line (while it will be smooth away from it). However the directions orthogonal to the line turn out analogous to the $t$ direction in the previous paragraph, i.e. the test function is actually not required to be smooth in these directions for the pairing to be defined. This allows to make sense of the formula (6.85) also in this case. We omit the details.

\subsubsection{Fixing points}

We would like to put the results of the previous section in the context of a general question of "fixing points" in a distribution. E.g. we know that the Minkowski 4-point function is a translationally invariant distribution. Using translation invariance we can always fix one of the 4 points to a given position, e.g. zero, and consider it as a distribution with respect to the remaining 3 positions. One could ask if one can do better than that, i.e. to fix $n$ points to given positions and consider the 4-point function as a distribution with respect to the remaining $4-n$ positions. Where the 4 -point function is real-analytic we can of course consider all four points as fixed.

Now, results of section 6.6.1 show that, if one excludes lightlike separations limiting to configurations having some fixed causal ordering, one can fix a conformal frame, i.e. fix three points to some fixed positions, and the fourth point to a position characterized by two conformal cross ratios, and consider the distribution as a distribution in only two variables (cross ratios). It is not clear if results of section 6.6.1 can be generalized to cover lightlike separations.

In some cases it is possible to argue that one can fix more than one point without using conformal invariance. E.g. we may always fix a consecutive pair of points, i.e. $\left(x_{k}, x_{k+1}\right)$, where $k=1,2$ or 3, to spacelike-separated positions in Minkowski space, while allowing the remaining two points to approach Minkowski limit from the forward tube. The proof of lemma 6.1 can be slightly modified to show that $|\rho|,|\bar{\rho}|<1$ for such configurations (see section 6.9 .1 below). Moreover, a powerlaw bound also holds, by a slight modification of the argument after eq. (6.73). ${ }^{56}$ Then our arguments show that the Minkowski 4-point function is a distribution with respect to the two unfixed coordinates, which depends analytically on the fixed coordinates. In this case the unfixed coordinates may have any causal orderings and also lightlike separation.

One interesting case is that of the double light cone (DLC) singularity, i.e. the region close to $x_{1}=0, x_{3}=\widehat{e}_{1}, x_{4}=\infty$, while $x_{2}$ on the light cones of $x_{1}, x_{3}$. Our results are the

\footnotetext{
${ }^{56}$ Since $S(c)=\infty$ in these cases, we cannot rely on (6.53). Instead we directly show powerlaw bounds on $S\left(\Upsilon_{1}\right), S\left(\Upsilon_{2}\right)$ defined in eq. (6.73). Then the powerlaw bound on $|\rho|,|\bar{\rho}|$ holds by lemma 6.6. For $k=1$, by fixing $\zeta_{3}=0$, and using lemmas 6.4 and 6.5 , we have $S\left(\Upsilon_{i}\right) \leqslant S\left(\zeta_{i}^{\prime}\right)+S\left(-\zeta_{4}^{\prime}\right) \leqslant S\left(x_{i 3}\right)^{3}+S\left(x_{34}\right)^{3}$ $(i=1,2)$. This is the desired powerlaw bound with respect to $x_{3}$ and $x_{4}$. For $k=2, S\left(\Upsilon_{1}\right)$ is bounded as for $k=1$, while for $S\left(\Upsilon_{2}\right)$ we argue as follows. Since $x_{2}$ and $x_{3}$ are spacelike separated, after fixing $\zeta_{3}=0, \zeta_{2}$ is a spacelike Minkowski point, hence so is $\zeta_{2}^{\prime}$, i.e. $\operatorname{Im}\left(\zeta_{4}^{\prime}+\zeta_{2}^{\prime}\right)=\operatorname{Im}\left(\zeta_{4}^{\prime}\right)$. Then by lemma 6.4 , $S\left(\Upsilon_{2}\right) \leqslant S\left(\zeta_{4}^{\prime}\right)+\left|\zeta_{2}^{\prime}\right| \leqslant S\left(\zeta_{4}\right)^{3}+\left|\zeta_{2}^{\prime}\right|$, which is the needed bound. Case $k=3$ follows by similar arguments or by mapping it to $k=1$ via $\left(x_{1}, x_{2}, x_{3}, x_{4}\right) \rightarrow\left(x_{1}^{\prime}=x_{4}^{\theta}, x_{2}^{\prime}=x_{3}^{\theta}, x_{3}^{\prime}=x_{2}^{\theta}, x_{4}^{\prime}=x_{1}^{\theta}\right)$ which maps $\rho$ and $\bar{\rho}$ are to their complex conjugates.
} 
first ones which establish the existence of the Wightman 4-point function in a neighborhood of DLC. However, there is a difference between restricting to one causal ordering near DLC or studying an open neighborhood of DLC which includes several causal orderings (see figure 10 in Conclusions). In the former case we can use directly the results of section 6.6.1 and represents the 4-point function as a distribution in two variables $z, \bar{z}$. In the latter case we can fix, by the above argument, two successive spacelike points $x_{3}$ and $x_{4}$. We are left with a distribution depending on $x_{1}, x_{2}$, i.e. $2 \times d$ coordinates. This distribution still satisfies conformal invariance Ward identities w.r.t. infinitesimal conformal transformations preserving $x_{2}$. It would be interesting to understand how this constrains the distribution at the DLC.

Although it is not directly related, we would also like to mention here the classic result of Borchers [74] which says that it is enough to smear Wightman functions $G_{M}\left(x_{1}, \ldots, x_{n}=0\right)$ with respect to the time variables only, i.e. integrating with respect to $h_{1}\left(x_{1}^{0}\right) \ldots h_{n-1}\left(x_{n-1}^{0}\right)$ where $h_{i} \in \mathcal{S}(\mathbb{R})$, after which they become $C^{\infty}$ functions in the remaining spatial variables $\mathbf{x}_{i}$. This result is valid in any QFT satisfying Wightman axioms. It holds because smearing in time, which acts as an energy cutoff, is effectively also a momentum cutoff because $|\mathbf{p}| \leqslant E$.

\subsection{Wightman positivity}

Recall that in section 3.1 we showed that CFT axioms imply OS reflection positivity for 4point functions. That discussion gives us access to OS states $|\mathcal{O}(x) \mathcal{O}(y)\rangle$ with $0>x^{0}>y^{0}$, with finite norm, and inner products measured by the Euclidean 4-point function. We know that these states belong to the CFT Hilbert space, i.e. can be arbitrarily well approximated in norm by states produced by inserting finite linear combinations of CFT local operators at one point in the half-space $x^{0}<0$, e.g. the south pole $x_{S}=(-1, \mathbf{0})$.

Now that we analytically continued the 4-point function, we can consider other states involving operators at complexified coordinates. We wish to prove that those states belong to the CFT Hilbert space and have a positive definite inner product. This can be shown by a robust argument, going back to Osterwalder and Schrader [2], section 4.3. The argument uses only OS positivity and the Fourier-Laplace representation, but not directly the CFT axioms.

We will consider two new kinds of states. First, states generated by a pair of Minkowski operators smeared with respect to an arbitrary Schwartz test function:

$$
\left|\Psi_{M}(F)\right\rangle=\int d x d y F(x, y)\left|\mathcal{O}\left(i x^{0}, \mathbf{x}\right) \mathcal{O}\left(i y^{0}, \mathbf{y}\right)\right\rangle
$$

and second, states generated by a pair of Euclidean operators at complexified time positions:

$$
\left|\mathcal{O}\left(x_{3}\right) \mathcal{O}\left(x_{4}\right)\right\rangle, \quad x_{i}=\left(\epsilon_{i}+i t_{i}, \mathbf{x}_{i}\right), \quad 0>\epsilon_{3}>\epsilon_{4} .
$$

The inner products of states (6.87) are given by integrals of the Minkowski 4-point function

$$
\left\langle\Psi_{M}\left(F_{1}\right) \mid \Psi_{M}\left(F_{2}\right)\right\rangle=\int d x G_{4}^{M}\left(x_{1}, x_{2}, x_{3}, x_{4}\right) \overline{F_{1}\left(x_{2}, x_{1}\right)} F_{2}\left(x_{3}, x_{4}\right),
$$


while the natural inner product on the states (6.88) is:

$$
\left\langle\mathcal{O}\left(x_{1}\right) \mathcal{O}\left(x_{2}\right) \mid \mathcal{O}\left(x_{3}\right) \mathcal{O}\left(x_{4}\right)\right\rangle=G_{4}\left(x_{2}^{\theta}, x_{1}^{\theta}, x_{3}, x_{4}\right),
$$

where the OS reflection operation extends to points with complex time coordinates by:

$$
x=(\varepsilon+i t, \mathbf{x}) \mapsto x^{\theta}=(-\varepsilon+i t, \mathbf{x}) .
$$

The states (6.87) also have a natural inner product $\left\langle\mathcal{O}\left(x_{1}\right) \mathcal{O}\left(x_{2}\right) \mid \Psi(F)\right\rangle$ with the OS states. ${ }^{57}$

We wish to show that all these new inner products are positive definite and, moreover, that the new states can be approximated in norm by the smeared OS 2-operator states at Euclidean positions. Note that the positive definiteness of (6.89) is precisely Wightman positivity for the 4-point case.

\subsubsection{Wightman states}

Let us start with (6.89). Rewriting the inner product in terms of $W\left(p_{1}, p_{2}, p_{3}\right)$, the (distributional) Fourier transform of $G_{4}^{M}$ with respect to $y_{k}=x_{k}-x_{k+1}$, we obtain

$$
\left\langle\Psi_{M}\left(F_{1}\right) \mid \Psi_{M}\left(F_{2}\right)\right\rangle=\int d p W\left(p_{1}, p_{2}, p_{3}\right)\left[\widehat{F}_{1}\left(p_{2}-p_{1}, p_{1}\right)\right]^{*} \widehat{F}_{2}\left(p_{2}-p_{3}, p_{3}\right) .
$$

We will also need the inner products of the (smeared) OS states

$$
|\Psi(H)\rangle=\int d x d y H(x, y)|\mathcal{O}(x) \mathcal{O}(y)\rangle
$$

where $H$ is any $C^{\infty}$ function compactly supported at $0>x^{0}>y^{0}$. Their inner products are given by

$$
\left\langle\Psi\left(H_{1}\right) \mid \Psi\left(H_{2}\right)\right\rangle=\int d x G_{4}^{E}\left(x_{1}, x_{2}, x_{3}, x_{4}\right) \overline{H_{1}\left(x_{2}^{\theta}, x_{1}^{\theta}\right)} H_{2}\left(x_{3}, x_{4}\right) .
$$

This can be expressed using the Fourier-Laplace representation (4.11). We obtain

$$
\left\langle\Psi\left(H_{1}\right) \mid \Psi\left(H_{2}\right)\right\rangle=\int d p W\left(p_{1}, p_{2}, p_{3}\right) \overline{g\left(H_{1}\right)\left(p_{2}, p_{1}\right)} g\left(H_{2}\right)\left(p_{2}, p_{3}\right),
$$

where $g(H)(p, q)$ is a Schwartz class function related to $H(x, y)$ as follows. First we form the function $h\left(y_{1}, y_{2}\right)=H\left(-y_{1},-y_{1}-y_{2}\right)$ which has support at $y_{1}^{0}, y_{2}^{0}>0$. Next we consider $\tilde{h}$, the Fourier-Laplace transform of $h\left(y_{1}, y_{2}\right)$ :

$$
\tilde{h}\left(p_{1}, p_{2}\right)=\int d y_{1} d y_{2} e^{-p_{1}^{0} y_{1}^{0}+i \mathbf{p}_{1} \cdot \mathbf{y}_{1}-p_{2}^{0} y_{2}^{0}+i \mathbf{p}_{2} \cdot \mathbf{y}_{2}} h\left(y_{1}, y_{2}\right) .
$$

Finally, $g(H)$ is an arbitrary Schwartz class function which coincides with $\tilde{h}$ inside the forward light cones. We also have an analogous formula for the inner product between states of two types:

$$
\left\langle\Psi(H) \mid \Psi_{M}(F)\right\rangle=\int d p W\left(p_{1}, p_{2}, p_{3}\right) \overline{g(H)\left(p_{2}, p_{1}\right)} \widehat{F}_{2}\left(p_{2}-p_{3}, p_{3}\right) .
$$

\footnotetext{
${ }^{57}$ We start from the analytically continued Euclidean 4-point function $G_{4}\left(x_{1}, x_{2}, x_{3}, x_{4}\right)$ and take the limit where $x_{1}, x_{2}$ are kept at fixed Euclidean positions, while $x_{3}, x_{4}$ approach the Minkowski space. By theorem 4.1, the limit is a distribution in $x_{3}, x_{4}$, and the inner product is its pairing with the test function $F$.
} 
At this point we recall lemma 4.2 from section 4.1. That lemma implies that Schwartz functions of the form $g(H)$ are dense in the Schwartz space. In particular, for any Schwartz $F$, we can find a sequence of functions $\left\{H_{r}\right\}_{r=1}^{\infty}$ such that $g\left(H_{r}\right)\left(p_{2}, p_{3}\right) \rightarrow \widehat{F}\left(p_{2}-p_{3}, p_{3}\right)$ in the Schwartz space. Then it follows from (6.92), (6.95), (6.97) that

$$
\begin{aligned}
\left\langle\Psi\left(H_{r}\right) \mid \Psi\left(H_{r}\right)\right\rangle & \rightarrow\left\langle\Psi_{M}(F) \mid \Psi_{M}(F)\right\rangle, \\
\left\langle\Psi\left(H_{r}\right) \mid \Psi_{M}(F)\right\rangle & \rightarrow\left\langle\Psi_{M}(F) \mid \Psi_{M}(F)\right\rangle .
\end{aligned}
$$

From the first equation we conclude that $\left\langle\Psi_{M}(F) \mid \Psi_{M}(F)\right\rangle \geqslant 0$, proving Wightman positivity. The two equations taken together imply that

$$
\left\langle\Psi\left(H_{r}\right)-\Psi_{M}(F) \mid \Psi\left(H_{r}\right)-\Psi_{M}(F)\right\rangle \rightarrow 0,
$$

i.e. OS states can approximate Wightman states in norm.

\subsubsection{OS states for complexified times}

Let us discuss next the states (6.88) obtained by putting operators at complexified time positions. In these states we don't take the limit to Minkowski space, so they are defined without smearing. Using the Fourier-Laplace representation, their inner product (6.90) is expressed as

$$
\begin{aligned}
\left\langle\mathcal{O}\left(x_{1}\right) \mathcal{O}\left(x_{2}\right) \mid \mathcal{O}\left(x_{3}\right) \mathcal{O}\left(x_{4}\right)\right\rangle & =G_{4}\left(x_{2}^{\theta}, x_{1}^{\theta}, x_{3}, x_{4}\right) \\
& =\int d p W\left(p_{1}, p_{2}, p_{3}\right) \overline{f_{x_{1}, x_{2}}\left(p_{2}, p_{1}\right)} f_{x_{3}, x_{4}}\left(p_{2}, p_{3}\right),
\end{aligned}
$$

where $f_{x, y}(p, q)$, where $0>\operatorname{Re}\left(x^{0}\right)>\operatorname{Re}\left(y^{0}\right)$, is any Schwartz function which agrees with

$$
e^{p^{0} x^{0}-i \mathbf{p} \cdot \mathbf{x}-q^{0}\left(x^{0}-y^{0}\right)+i \mathbf{q} \cdot(\mathbf{x}-\mathbf{y})} .
$$

for $p, q$ in the forward light cone (where this function is exponentially decreasing) and extends it somehow outside the light cones (it does not matter how because $W$ has support in the forward light cones).

Since $f_{x, y}$ is a Schwartz function, it can be approximated by Schwartz functions of the form $g(H)$ as in the preceding subsection. This implies that non-smeared complexified OS states can be approximated in norm by Euclidean OS states smeared with compactly supported test functions. In particular, the inner product (6.90) is positive definite, providing an extension of pointwise OS positivity to complexified times:

$$
G_{4}\left(y^{\theta}, x^{\theta}, x, y\right) \geqslant 0, \quad\left(0>\operatorname{Re} x^{0}>\operatorname{Re} y^{0}\right) .
$$

As usual, positive-definite inner product implies a Cauchy-Schwarz inequality for the complexified times:

$$
\left|G_{4}\left(x_{1}, x_{2}, x_{3}, x_{4}\right)\right|^{2} \leqslant G_{4}\left(x_{1}, x_{2}, x_{2}^{\theta}, x_{1}^{\theta}\right) G_{4}\left(x_{4}^{\theta}, x_{3}^{\theta}, x_{3}, x_{4}\right),
$$

valid for $\operatorname{Re} x_{1}^{0}>\operatorname{Re} x_{2}^{0}>0>\operatorname{Re} x_{3}^{0}>\operatorname{Re} x_{4}^{0}$. The analogues of these properties for conformal blocks will be useful in section 7 . 
Remark 6.3. We can extend the reflection operation further for points with complexified both time and space coordinates, as

$$
x=(\varepsilon+i t, \mathbf{x}+i \mathbf{y}) \mapsto x^{\theta}=(-\varepsilon+i t, \mathbf{x}-i \mathbf{y}) .
$$

With this definition, we can show by the same arguments as above that $G_{4}\left(y^{\theta}, x^{\theta}, x, y\right) \geqslant 0$ (pointwise OS positivity) remains true for $0 \succ\left(\operatorname{Re} x^{0}, \operatorname{Im} \mathbf{x}\right) \succ\left(\operatorname{Re} y^{0}, \operatorname{Im} \mathbf{y}\right)$ where $\eta_{1} \succ \eta_{2}$ means $\eta_{1}-\eta_{2} \succ 0$ (i.e. in the forward light cone). We can then show that the states $|\mathcal{O}(x) \mathcal{O}(y)\rangle$ make sense for such $x, y$ and can be approximated in norm by integrated Euclidean OS states.

\subsection{Wightman clustering}

\subsection{1 $2+2$ split}

In this section we will derive clustering (2.4) for Wightman 4-point functions (see [2], section 4.4). As in section 3.3 for the OS case, we will consider $2+2$ and $3+1$ splits separately. The property we need to prove in the $2+2$ case can be written conveniently in the language of Wightman states $\left|\Psi_{M}(F)\right\rangle$, at our disposal by the discussion in section 6.7 :

$$
\left\langle\Psi_{M}\left(F_{1}\right) \mid \Psi_{M}\left(U_{\lambda a} F_{2}\right)\right\rangle \rightarrow\left\langle\Psi_{M}\left(F_{1}\right) \mid \Omega\right\rangle\left\langle\Omega \mid \Psi_{M}\left(F_{2}\right)\right\rangle
$$

as $\lambda \rightarrow \infty$ for any spacelike vector $a$ and any Schwartz test functions $F_{1}, F_{2}$, where $U_{\lambda a}$ is translation: $\left(U_{\lambda a} F_{2}\right)(x, y)=F_{2}(x-\lambda a, y-\lambda a)$, and $\Omega$ is the vacuum state corresponding to inserting the unit operator. By Lorentz invariance it's enough to prove this for $a=(0, \mathbf{a})$, purely spatial vector. In section 3.3 we showed the OS clustering, which we can also write using the integrated OS states (6.93), as

$$
\left\langle\Psi\left(H_{1}\right) \mid \Psi\left(U_{\lambda a} H_{2}\right)\right\rangle \rightarrow\left\langle\Psi\left(H_{1}\right) \mid \Omega\right\rangle\left\langle\Omega \mid \Psi\left(H_{2}\right)\right\rangle .
$$

As explained in section 6.7 , we can find states $\left|\Psi\left(H_{1}\right)\right\rangle$ and $\left|\Psi\left(H_{2}\right)\right\rangle$ which approximate $\left|\Psi_{M}\left(F_{1}\right)\right\rangle$ and $\left|\Psi_{M}\left(F_{2}\right)\right\rangle$ in norm within any $\varepsilon>0$. Moreover it's obvious from that construction that the norm is invariant under shifts in purely spatial direction (i.e. the operator $U_{\lambda a}$ is unitary). Hence we have $\left\|\Psi\left(U_{\lambda a} H_{2}\right)-\Psi_{M}\left(U_{\lambda a} F_{2}\right)\right\|=\left\|\Psi\left(H_{2}\right)-\Psi_{M}\left(F_{2}\right)\right\| \leqslant$ $\varepsilon$ for any $\lambda$. By these properties, (6.106) implies (6.105). ${ }^{58}$

\subsection{2 $3+1$ split}

Let us first restate the Euclidean $3+1$ clustering argument from section 3.3 in a somewhat more explicit form, and specializing to scalars. So let $\varphi\left(x_{1}\right), \chi\left(x_{2}, x_{3}, x_{4}\right)$ be two smooth functions with compact support ${ }^{59}$

$$
\operatorname{supp}(\varphi) \subset\left\{x_{1}^{0}>0\right\}, \quad \operatorname{supp}(\chi) \subset\left\{0>x_{2}^{0}>x_{3}^{0}>x_{4}^{0}\right\} .
$$

\footnotetext{
${ }^{58}$ Indeed we have $\left|\left\langle\Psi_{M}\left(F_{1}\right) \mid \Psi_{M}\left(U_{\lambda a} F_{2}\right)\right\rangle-\left\langle\Psi\left(H_{1}\right) \mid \Psi\left(U_{\lambda a} H_{2}\right)\right\rangle\right| \leqslant C \varepsilon$ with some $C$ independent of $\lambda$. Now passing to the limit $\lambda \rightarrow \infty$ and using (6.106) we obtain $\lim \sup _{\lambda \rightarrow \infty}\left\langle\Psi_{M}\left(F_{1}\right) \mid \Psi_{M}\left(U_{\lambda a} F_{2}\right)\right\rangle \leqslant$ $\left\langle\Psi_{M}\left(F_{1}\right) \mid \Omega\right\rangle\left\langle\Omega \mid \Psi_{M}\left(F_{2}\right)\right\rangle+C^{\prime} \varepsilon$, and an analogous lower bound on lim $\inf _{\lambda \rightarrow \infty}$. Since $\varepsilon>0$ is arbitrary we obtain $(6.105)$.

${ }^{59}$ For simplicity, in this section we prove clustering for compactly-supported, as opposed to Schwartz, test functions. We expect that it should be possible to find a proof for Schwartz test functions as well. In any case, the most natural proof would use positivity and the OPE similarly to $2+2$ split, provided positivity for higher-point functions is proven (which we don't do in this paper).
} 
We would like to show

$$
\lim _{\lambda \rightarrow \infty}\left(G, \varphi_{\lambda} \otimes \chi\right)=0, \quad \varphi_{\lambda}:=\varphi\left(\cdot-\lambda \widehat{e}_{1}\right)
$$

where $G=G_{4}^{E}$ is the Euclidean 4-point function of four identical scalars, and $\widehat{e}_{1}$ is the $x^{1}$ unit vector. The main idea is that we can find a conformal transformation which moves the point at infinity as well as all the other points to some finite positions. The suppression of the integral then comes from the Jacobian of this transformation. Consider a special conformal transformation $f(x)=\frac{x^{\mu}+x^{2} b^{\mu}}{1+2 x \cdot b+x^{2} b^{2}}=\mathcal{J} \circ T_{b} \circ \mathcal{J}$, where $\mathcal{J}$ is inversion and $T_{b}$ is a translation by $b=\widehat{e}_{1}$. We have $f\left(-\widehat{e}_{1}\right)=\infty$, while $f$ is non-singular on $\operatorname{supp}\left(\varphi_{\lambda}\right)$ and $\operatorname{supp}(\chi)$. We also have $f(\infty)=\widehat{e}_{1}$. By conformal invariance we have (compare (6.78)) $(G, \Phi)=\left(G, \Phi^{f}\right)$ where $\Phi^{f}\left(x_{1}, \ldots, x_{4}\right)=\Phi\left(f^{-1}\left(x_{1}\right), \ldots, f^{-1}\left(x_{4}\right)\right) \prod_{i=1}^{4} J\left(f^{-1}\left(x_{i}\right)\right)^{\Delta_{\mathcal{O}}-d}$, where $J(x)=\frac{1}{1+2 x \cdot b+x^{2} b^{2}}$. We apply this equation with $\Phi=\varphi_{\lambda} \otimes \chi$. The function $\chi$ is mapped by this transformation to some smooth function. Suppression of the integral in the limit $\lambda \rightarrow \infty$ will come from the transformation of $\varphi_{\lambda}$, which is mapped to

$$
\varphi_{\lambda}^{f}\left(x_{1}\right):=\varphi\left(f^{-1}\left(x_{1}\right)-\lambda \widehat{e}_{1}\right) J\left(f^{-1}\left(x_{1}\right)\right)^{\Delta_{\mathcal{O}}-d} .
$$

Namely we have

$$
\left|\left(G, \varphi_{\lambda}^{f} \otimes \chi^{f}\right)\right| \leqslant C(\lambda) I, \quad I=\int d x_{1}\left|\varphi_{\lambda}^{f}\left(x_{1}\right)\right|, \quad C(\lambda)=\sup _{x_{1} \in \operatorname{supp} \varphi_{\lambda}^{f}}\left|\left(G\left(x_{1}, \cdot\right), \chi^{f}\right)\right| .
$$

The function $\varphi_{\lambda}^{f}$ is nonzero for $f^{-1}\left(x_{1}\right) \in \operatorname{supp}(\varphi)+\lambda \widehat{e}_{1}$, which is a point near infinity for $\lambda$ large. We conclude that $\varphi_{\lambda}^{f}$ is supported in a small neighborhood, order $1 / \lambda$, of $f(\infty)=\widehat{e}_{1}$. Since $G$ is real-analytic at nonzero point separation, this implies that $C(\lambda)$ is bounded by some constant for $\lambda \geqslant \lambda_{0}$. To compute $I$, we do the change of variables $x_{1}=f(y)$ :

$$
I=\int d y\left|\varphi\left(y-\lambda \widehat{e}_{1}\right)\right| J(y)^{\Delta_{\mathcal{O}}} \sim \frac{\text { const }}{\lambda^{2 \Delta_{\mathcal{O}}}} .
$$

This finishes the proof of Euclidean 3+1 clustering, eq. (6.108).

Let us proceed next to show Wightman $3+1$ clustering. We will show the same equation as (6.108), namely

$$
\lim _{\lambda \rightarrow+\infty}\left(G, \varphi_{\lambda} \otimes \chi\right)=0,
$$

where now $G=G_{4}^{M}$ is the Minkowski 4-point function, which is a tempered distribution, and $\varphi\left(x_{1}\right)$ and $\chi\left(x_{2}, x_{3}, x_{4}\right)$ are arbitrary compactly supported test functions (i.e. no support requirements analogous to (6.107)). ${ }^{60}$ The proof will be based on the same idea of moving the point at infinity to a finite position, paying attention to $G$ now being distribution, and to the requirement (6.79) on invariance under finite Minkowski conformal transformations.

\footnotetext{
${ }^{60}$ The method described below cannot be straightforwardly generalized to the case of Schwartz test functions.
} 


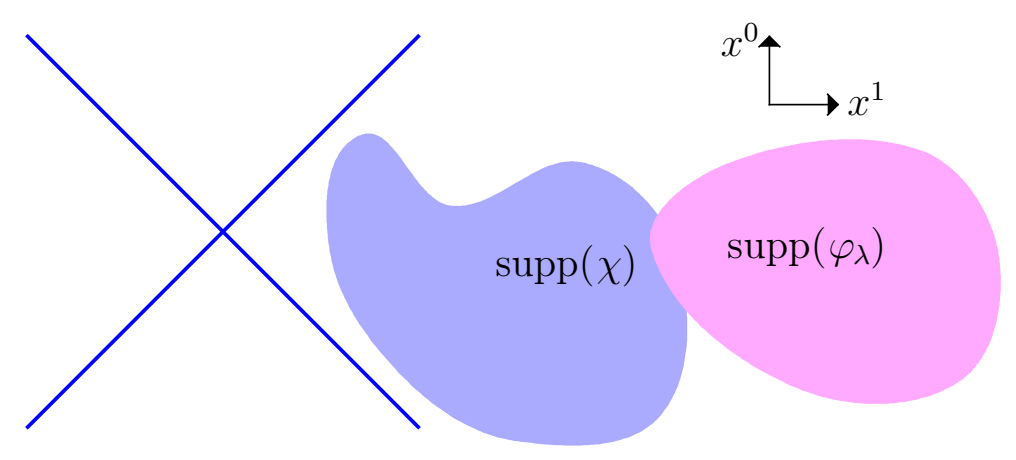

Figure 5. Location of supports of $\varphi_{\lambda}$ and $\chi$ with respect to the singularity light cone of $f$.

We will use the same transformation $f(x)=\frac{x^{\mu}+x^{2} b^{\mu}}{1+2 x \cdot b+x^{2} b^{2}}, b=\widehat{e}_{1}$. By translation invariance, we may assume that $\operatorname{supp}(\chi)$ lies at larger $x^{1}$ values than of the singularity light cone $x^{0}= \pm\left|\mathbf{x}+\widehat{e}_{1}\right|$ of this transformation (see section 6.6). For sufficiently large $\lambda$, $\operatorname{supp}\left(\varphi_{\lambda}\right)$ will also satisfy this condition. As we scale $b$ to zero to connect $f$ to the identity, the singularity light cone moves away to infinity along the negative $x^{1}$ direction, without touching $\operatorname{supp}\left(\varphi_{\lambda}\right)$ nor $\operatorname{supp}(\chi)$, see figure 5. Hence requirement (6.79) is satisfied and we may apply invariance (6.78), which says $\left(G, \varphi_{\lambda} \otimes \chi\right)=\left(G, \varphi_{\lambda}^{f} \otimes \chi^{f}\right)$.

Now, using translation invariance of the 4-point function, $G\left(x_{1}, x_{2}, x_{3}, x_{4}\right)=\tilde{G}\left(x_{2}-\right.$ $\left.x_{1}, x_{3}-x_{1}, x_{4}-x_{1}\right)$ we may write

$$
\left(G, \varphi_{\lambda}^{f} \otimes \chi^{f}\right)=\int d x_{1} \varphi_{\lambda}^{f}\left(x_{1}\right) F\left(x_{1}\right),
$$

where $F\left(x_{1}\right)=\left(\tilde{G}, T_{x_{1}} \cdot \chi^{f}\right)$ and $T_{a}$ is a translation. $\tilde{G}$ is a distribution, but since translation is a continuous operation in the space of test functions, we know that $F\left(x_{1}\right)$ is a continuous function of $x_{1}$. When $\lambda$ goes to $+\infty$, the support of $\varphi_{\lambda}^{f}$ shrinks to the point $\widehat{e}_{1} \cdot{ }^{61}$ Hence for $\lambda \geqslant \lambda_{0}$ we can bound $|F|$ on $\operatorname{supp}\left(\varphi_{\lambda}^{f}\right)$ by a constant, and estimate (6.113) in absolute value by const $\times \int d x_{1}\left|\varphi_{\lambda}^{f}\left(x_{1}\right)\right|$. This remaining integral is computed via the change of variables as the Euclidean one, and goes to zero as $\lambda^{-2 \Delta_{\mathcal{O}}}$, completing the proof.

\subsection{Local commutativity}

Let us show that the constructed Minkowski correlators satisfy local commutativity. This follows by a robust argument which uses only Lorentz invariance, analyticity in the forward tube, existence of the boundary distribution, together with real analyticity and permutation symmetry of the Euclidean correlators away from coincident points (OS [2], section 4.5). Here for completeness we will provide this argument for $n$-point functions which is its natural setting. In section 6.9 .1 below we will make some remarks specific to CFT 4point functions.

So, we start from the Euclidean correlator $G^{E}\left(x_{1}, \ldots, x_{n}\right)$ at $x_{1}^{0}>x_{2}^{0}>\cdots>x_{n}^{0}$ and its analytic continuation $G\left(x_{1}, \ldots, x_{n}\right)$ to the forward tube $\mathcal{T}_{n}$ which is the set of points

\footnotetext{
${ }^{61}$ It is important for the argument that, as one can easily check, $\operatorname{supp}\left(\varphi_{\lambda}^{f}\right) \operatorname{shrinks}$ to a compact set (in fact, a point) and not, say, spreads out along some light cone.
} 
$x_{k} \in \mathbb{C}^{d}$ such that their differences $y_{k}=x_{k}-x_{k+1}$ satisfy $\operatorname{Re} y_{k}^{0}>\left|\operatorname{Im} \mathbf{y}_{k}\right|$ or equivalently $\eta_{k} \succ 0$ in terms of $\zeta_{k}=\left(i y_{k}^{0}, \mathbf{y}_{k}\right)=\xi_{k}+i \eta_{k}, \xi_{k}, \eta_{k} \in \mathbb{R}^{1, d-1}$. We will write $G$ instead of $G_{n}$. We know by theorem 4.1 that this analytic continuation is invariant under Lorentz transformations $\zeta_{k} \rightarrow \Lambda \zeta_{k}$ where $\Lambda \in L_{+}^{\uparrow}$, the identity component of the real Lorentz group. Since $G$ is translationally invariant, it depends only on $\zeta_{k}$, and we will abuse of notation by sometimes writing $G\left(\zeta_{1}, \ldots, \zeta_{n-1}\right)$ and $\left(\zeta_{1}, \ldots, \zeta_{n-1}\right) \in \mathcal{T}_{n}$ instead of $G\left(x_{1}, \ldots, x_{n}\right)$ and $\left(x_{1}, \ldots, x_{n}\right) \in \mathcal{T}_{n}$.

Step 1. We will extend domain of analyticity of $G$ using the complex Lorentz group $L(\mathbb{C})$, defined as the set of complex matrices $A$ preserving the Minkowski metric, i.e. $A^{T} g A=g$ where $g=\operatorname{diag}(-1,1 \ldots 1)$. We will only need the component of $L(\mathbb{C})$ connected to the identity, denoted $L_{+}(\mathbb{C})$. For any $\Lambda \in L_{+}(\mathbb{C})$ consider the equation

$$
G\left(\zeta_{1}, \ldots, \zeta_{n-1}\right)=G\left(\Lambda^{-1} \zeta_{1}, \ldots, \Lambda^{-1} \zeta_{n-1}\right)
$$

The two sides of this equation coincide for real $\Lambda \in L_{+}^{\uparrow}$ (by Lorentz invariance of $G_{n}$ ), and hence by analyticity in the components of $\Lambda$ also for complex $\Lambda \in L_{+}(\mathbb{C})$, at least for $\Lambda$ close to 1 . In other words, eq. (6.114) is just an identity if $\Lambda \approx 1$ and the arguments of $G_{n}$ on both sides are in the forward tube. But a general $\Lambda \in L_{+}(\mathbb{C})$ does not preserve the forward tube. For such $\Lambda$, eq. (6.114) extends analytically $G$ from the forward tube to the set

$$
\mathcal{T}_{n}^{\prime}=\bigcup_{\Lambda \in L_{+}(\mathbb{C})} \Lambda \cdot \mathcal{T}_{n}
$$

called the extended tube. The Bargmann-Hall-Wightman theorem shows that no further topological obstructions arise in this analytic continuation; see [75], p. 78 for details. Call this extension $\tilde{G}$.

Step 2. Let us consider $\tilde{G}\left(x_{1}, \ldots, x_{n}\right)$ for

$$
\epsilon_{1}>\ldots>\epsilon_{k-1}>0>\epsilon_{k+2}>\ldots>\epsilon_{n},
$$

while assuming that $\epsilon_{k}, \epsilon_{k+1}$ are near zero and much smaller than other $\epsilon_{i}$ 's, and $\left|t_{k}-t_{k+1}\right|<$ $\left|\mathbf{x}_{k}-\mathbf{x}_{k+1}\right|, \mathbf{x}_{k}, \mathbf{x}_{k+1}$ real, so that $x_{k}-x_{k+1}$ approaches a spacelike separation. For $\epsilon_{k}>\epsilon_{k+1}$ this configuration is in the forward tube, so we know $\tilde{G}$ is analytic there and agrees with $G\left(x_{1}, \ldots, x_{n}\right)$. Let us show that the configurations with $\epsilon_{k}<\epsilon_{k+1}$ are in the extended tube. We may set $x_{k+1}=0$ for this argument, so that

$$
\zeta_{k}=\left(t_{k}+i \epsilon_{k}, \mathbf{x}_{k}\right) .
$$

We may assume without loss of generality that $\mathbf{x}_{k}=\left(x_{k}^{1}, 0, \ldots 0\right), x_{k}^{1}>\left|t_{k}\right|$. Then acting on $\zeta_{k}$ with the complexified Lorentz transformation

$$
\Lambda_{\theta}=\left(\begin{array}{cc}
\cosh (i \theta) & \sinh (i \theta) \\
\sinh (i \theta) & \cosh (i \theta)
\end{array}\right) \in L_{+}(\mathbb{C}),
$$

with small $\theta$ we get, using $\Lambda_{\theta} \approx\left(\begin{array}{cc}1 & i \theta \\ i \theta & 1\end{array}\right), \zeta_{k}^{\prime}=\Lambda_{\theta} \zeta_{k} \approx\left(t_{k}, x_{k}^{1}\right)+i\left(\theta x_{k}^{1}+\epsilon_{k}, \theta t_{k}\right)$, and thus $\eta_{k}^{\prime} \approx\left(\theta x_{k}^{1}+\epsilon_{k}, \theta t_{k}\right)$. If $\epsilon_{k}$ is negative but very small, we can can achieve $\eta_{k}^{\prime} \succ 0$ by choosing 


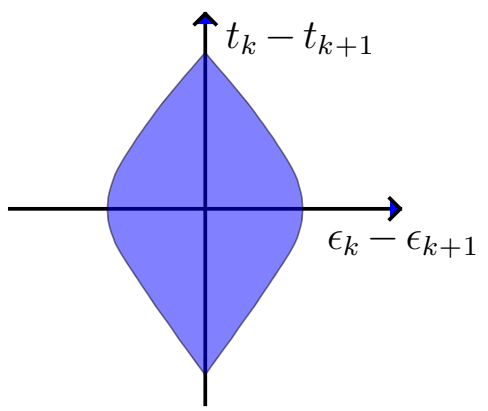

Figure 6. Projection of the set $\mathcal{Q}_{n, k}$, where the function $\tilde{G}$ is holomorphic, to the plane $\left(\epsilon_{k}-\right.$ $\left.\epsilon_{k+1}, t_{k}-t_{k+1}\right)$. The vertical extent of this region is determined by the condition $\left|t_{k}-t_{k+1}\right|<$ $\left|\mathbf{x}_{k}-\mathbf{x}_{k-1}\right|$. The horizontal extent is determined, among other things, by the condition that $\epsilon_{k}, \epsilon_{k+1}$ have to be much smaller that all the other $\epsilon_{i}^{\prime}$ 's.

an appropriate small $\theta$. We need $\theta$ small so that all the other $\zeta_{i}^{\prime}$ remain in the forward light cone, and this will work because we are assuming that $\epsilon_{k}$ is very much smaller than all the other $\epsilon_{i}$ 's.

The bottom line is that the extended tube contains an open set of configurations as above, with $\left|t_{k}-t_{k+1}\right|<\left|\mathbf{x}_{k}-\mathbf{x}_{k+1}\right|$ and $\epsilon_{k}, \epsilon_{k+1}$ small, with $\epsilon_{k}-\epsilon_{k+1}$ of any sign. Let us call this set $\mathcal{Q}_{n, k}$. By restricting this set a bit, we may assume that $\mathcal{Q}_{n, k}$ is invariant under permutations of $x_{k}$ and $x_{k+1}$. By Step 1 we know that function $\tilde{G}$ is holomorphic in the extended tube and hence also in $\mathcal{Q}_{n, k}$. In particular, it is analytic if we set $\epsilon_{k}=\epsilon_{k+1}=0$. This already has an interesting consequence: the Minkowski correlator is analytic with respect to a pair of spacelike-separated points (while it remains a distribution with respect to all the other points). The projection of $\mathcal{Q}_{n, k}$ to the plane $\left(\epsilon_{k}-\epsilon_{k+1}, t_{k}-t_{k+1}\right)$ is shown schematically in figure 6 .

The set $\mathcal{Q}_{n, k}$ contains real configurations (horizontal axis in figure 6 , setting other $t_{i} \rightarrow 0$ as well). Restriction of $\tilde{G}$ to the real part of $\mathcal{Q}_{n, k}$ agrees with the Euclidean correlator $G^{E}$. (They agree for $\epsilon_{k}>\epsilon_{k+1}$ by construction and for $\epsilon_{k}<\epsilon_{k+1}$ by the uniqueness of analytic continuation. Recall that the Euclidean correlator $G^{E}$ is real analytic everywhere away from coincident points, i.e. for $\epsilon_{k}-\epsilon_{k+1}$ of any sign as long as $\mathbf{x}_{k} \neq \mathbf{x}_{k-1}$.) One consequence of this fact is that $\tilde{G}$ restricted to the real part $\mathcal{Q}_{n, k}$ is permutation invariant w.r.t. $x_{k} \leftrightarrow x_{k+1}$ :

$$
\tilde{G}\left(\ldots x_{k}, x_{k+1} \ldots\right)=\tilde{G}\left(\ldots x_{k+1}, x_{k} \ldots\right),
$$

because the Euclidean correlator has this property. Finally, since $\mathcal{Q}_{n, k}$ is connected to the real configurations (see figure 6), we conclude that permutation invariance (6.119) holds everywhere in $\mathcal{Q}_{n, k} \cdot{ }^{62}$

We now see the meaning of $\tilde{G}$ for configurations with $\epsilon_{k}<\epsilon_{k+1}$. Via permutation invariance (6.119), such configurations are mapped to the forward tube and hence can be evaluated as $G$ for the permuted configurations.

\footnotetext{
${ }^{62}$ In fact, $\tilde{G}$ can be extended to a single-valued holomorphic function on the "permuted extended tube" $\bigcup_{\pi \in S^{n}} \pi \mathcal{T}_{n}^{\prime}$, and satisfied permutation invariance (6.119) on this large set. See [75], appendix II, [76] and [77], section 9.D. However for our purposes analyticity and permutation invariance on $\mathcal{Q}_{n, k}$ will suffice.
} 
Step 3. We are now ready to show local commutativity. We have to prove that boundary value limits of two holomorphic functions agree:

$$
\lim _{\epsilon_{i} \rightarrow 0} G\left(\ldots x_{k}, x_{k+1} \ldots\right)=\lim _{\epsilon_{i} \rightarrow 0} G\left(\ldots x_{k+1}, x_{k} \ldots\right)
$$

when approaching a Minkowski configuration in which $x_{k}-x_{k+1}$ is spacelike. Note that, by the original definition, the two limits are from different forward tubes: the first one must respect the condition $\epsilon_{k}>\epsilon_{k+1}$, while the second $\epsilon_{k+1}>\epsilon_{k}$. By theorem 4.1, Part 3, we can take the limits $\epsilon_{i} \rightarrow 0$ in any order, so let us send $\epsilon_{k}, \epsilon_{k+1} \rightarrow 0$ first, while keeping other $\epsilon_{i}$ fixed for the moment. For very small $\epsilon_{k}, \epsilon_{k+1}$, the configurations on both sides will be in $\mathcal{Q}_{n, k}$ where both sides are restrictions of the function $\tilde{G}$ analytic around $\epsilon_{k}, \epsilon_{k+1}=0$ and satisfying permutation invariance (6.119). It follows that the two sides of (6.120) agree in the limit $\epsilon_{k}, \epsilon_{k+1} \rightarrow 0$. Sending the remaining $\epsilon_{i} \rightarrow 0$ we recover the local commutativity.

\subsubsection{Local commutativity for CFT 4-point functions}

In this paper we analytically continued the CFT 4-point function $\left\langle\mathcal{O}\left(x_{1}\right) \mathcal{O}\left(x_{2}\right) \mathcal{O}\left(x_{3}\right) \mathcal{O}\left(x_{4}\right)\right\rangle$ to the forward tube using $\rho, \bar{\rho}$ coordinates. We would like to indicate here that this provides an alternative path to understanding local commutativity. We have shown previously that $0<|\rho|,|\bar{\rho}|<1$ in the forward tube. Since the extended tube is obtained from the forward tube by complexified Lorentz transformations and $\rho, \bar{\rho}$ are invariant under such transformations, it follows that $0<|\rho|,|\bar{\rho}|<1$ also in the extended tube. Below we will show this explicitly for the configurations used in the proof of local commutativity. We consider separately $k=1$ and $k=2(k=3$ being analogous to $k=1)$.

$\boldsymbol{k}=\mathbf{1}$ : here $x_{1}, x_{2}$ approach spacelike-separated Minkowski points. We know that $|\rho|,|\bar{\rho}|<1$ in $\mathcal{D}_{4}, \epsilon_{1}>\epsilon_{2}>\epsilon_{3}>\epsilon_{4}$. Extended tube analyticity suggests that this must remain true also for $\epsilon_{1}=\epsilon_{2}>\epsilon_{3}>\epsilon_{4}$. Indeed, this follows from critical rereading of the proof of lemma 6.1 (section 6.4, eq. (6.38) and below). (That proof does not use the condition $\eta_{1} \succ \eta_{2}$ but only $\eta_{1}, \eta_{2} \succ 0 .^{63}$ ) It is also important for analyticity that $\rho, \bar{\rho}$ not vanish. In the forward tube $\rho, \bar{\rho}$ do not vanish because $x_{i j}^{2} \neq 0, i<j$ (lemma 5.1). When $\epsilon_{1}=\epsilon_{2}$ we have $x_{12}^{2}>0$ (spacelike separation), hence also nonzero. These observations show that the CFT 4-point function can be analytically extended, using the $\rho, \bar{\rho}$ expansion, to a neighborhood of points with $\epsilon_{1}=\epsilon_{2}>0>\epsilon_{3}>\epsilon_{4}, x_{12}^{2}>0$, in agreement with the general QFT arguments given above.

Let us now permute the first two points: $\left(\epsilon_{1}+i t_{1}, \mathbf{x}_{1}\right) \leftrightarrow\left(\epsilon_{2}+i t_{2}, \mathbf{x}_{2}\right)$. In the Euclidean region, this transformation maps $\rho \rightarrow-\rho, \bar{\rho} \rightarrow-\bar{\rho}$ and leaves the 4-point function of identical scalars invariant because the expansion (6.11) contains only even $m$. The same transformation remains true for complexified times for spacelike separation. Taking the limit $\epsilon_{1}, \epsilon_{2} \rightarrow 0$, we recover local commutativity very explicitly.

\footnotetext{
${ }^{63} \mathrm{An}$ alternative argument is as follows. In section 7 we will show the Cauchy-Schwarz inequality for $\rho, \bar{\rho}$, theorem 7.2, which bounds $\rho, \bar{\rho}$ for any configuration in the forward tube with $\epsilon_{1}>\epsilon_{2}>0>\epsilon_{3}>\epsilon_{4}$ in terms of $\rho, \bar{\rho}$ of "reflection-symmetric" configurations having $\epsilon_{3}=-\epsilon_{2}, \epsilon_{4}=-\epsilon_{1}$. The proof of lemma 7.1, eq. (7.7) shows that $\rho, \bar{\rho}$ remain less than 1 for the latter configurations in the limit $\epsilon_{1} \rightarrow \epsilon_{2}$.
} 
$\boldsymbol{k}=2:$ now we are interested in the limit $\epsilon_{2} \rightarrow \epsilon_{3}$ from inside $\epsilon_{1}>\epsilon_{2}>\epsilon_{3}>\epsilon_{4}{ }^{64}$ As for $k=1$, critical rereading of the proof of lemma 6.1 shows that $|\rho|,|\bar{\rho}|$ remain less than 1 . (We put in that proof $\zeta_{2}=\xi_{2}+i \eta_{2}, \xi_{2}=\left(t_{2}, \mathbf{x}_{2}\right)$ spacelike, and $\eta_{2}=\left(\epsilon_{2}, \mathbf{0}\right), \epsilon_{2}>0$. The proof does not use the condition $\eta_{2}^{\prime} \succ 0$ but only $\eta_{24}^{\prime} \succ 0$. The latter condition remains true for $\epsilon_{2} \rightarrow \epsilon_{3}=0$, as $\zeta_{2}^{\prime}$ goes to a finite real vector.) Hence, the CFT 4-point function can be analytically extended, using the $\rho, \bar{\rho}$ expansion, to a neighborhood of points with $\epsilon_{1}>\epsilon_{2}=\epsilon_{3}>\epsilon_{4}, x_{23}^{2}<0$.

To finish the proof of local commutativity, we fall back on the general argument, appealing to the permutation invariance of the (real-analytic) CFT 4-point function under $x_{2} \leftrightarrow x_{3}$. (Unlike for $k=1$, the s-channel OPE expansion (6.11) cannot be used to make this step more explicit, as it does not manifestly have this invariance.)

\subsection{Generalization to non-identical scalars}

In the previous subsections we proved that the 4-point function of identical scalars has analytic continuation to the forward tube $\mathcal{T}_{4}$, and its boundary value in the Minkowski region is a tempered distribution. Then Minkowski conformal invariance, Wightman positivity, Wightman clustering and local commutativity follow from their Euclidean analogues.

In this section we will indicate how to generalize analytic continuation and temperedness to 4-point functions of non-identical scalars. The proof of the other properties is the same as in the case of identical scalars.

We consider the 4-point function of scalar primary operators $\mathcal{O}_{i}$ with scaling dimensions $\Delta_{i}$,

$$
\begin{aligned}
G_{1234}^{E}\left(c_{E}\right) & :=\left\langle\mathcal{O}_{1}\left(x_{1}\right) \mathcal{O}_{2}\left(x_{2}\right) \mathcal{O}_{3}\left(x_{3}\right) \mathcal{O}_{4}\left(x_{4}\right)\right\rangle \\
& =\frac{1}{\left(x_{12}^{2}\right)^{\frac{\Delta_{1}+\Delta_{2}}{2}}\left(x_{34}^{2}\right)^{\frac{\Delta_{3}+\Delta_{4}}{2}}}\left(\frac{x_{24}^{2}}{x_{14}^{2}}\right)^{\frac{\Delta_{1}-\Delta_{2}}{2}}\left(\frac{x_{14}^{2}}{x_{13}^{2}}\right)^{\frac{\Delta_{3}-\Delta_{4}}{2}} g_{1234}\left(c_{E}\right),
\end{aligned}
$$

which reduces to (6.1) when $\Delta_{i}$ 's are identical. The analytic continuation of the prefactor to the forward tube $\mathcal{T}_{4}$ is straightforward. The function $g_{1234}\left(c_{E}\right)$ only depends on the conformal equivalence class of $c_{E}$, i.e. $g_{1234}\left(c_{E}\right)=g_{1234}\left(\rho\left(c_{E}\right), \bar{\rho}\left(c_{E}\right)\right)$. By the similar argument to that in section 6.2 , the function $g_{1234}\left(c_{E}\right)$ has the following series expansion

$$
g_{1234}\left(c_{E}\right)=\left[\frac{(1-\rho)(1-\bar{\rho})}{(1+\rho)(1+\bar{\rho})}\right]^{\frac{\Delta_{1}-\Delta_{2}-\Delta_{3}+\Delta_{4}}{2}} \sum_{\delta, m} a_{12}(\delta, m) a_{\overline{43}}(\delta, m)^{*} r^{\delta} e^{i m \theta}, \quad \rho\left(c_{E}\right)=r e^{i m \theta},
$$

where the sum runs over a discrete set of pairs $(\delta, m)$ with $\delta \geqslant 0, m \in \mathbb{Z}$ (not necessarily even for non-identical scalars), $|m| \leqslant \delta$. Analogously to the case of identical scalars, the

\footnotetext{
${ }^{64}$ The discussion on the local commutativity of this type can also be found in the study of causality in a shockwave background (see section 5 of [4]). In [4], the 2-point function in a shockwave background is defined by $\langle\mathcal{O}(x) \mathcal{O}(y)\rangle_{\Psi}:=\frac{\langle\Psi(i \delta) \mathcal{O}(x) \mathcal{O}(y) \Psi(-i \delta)\rangle}{\langle\Psi(i \delta) \Psi(-i \delta)\rangle}$, where $x, y$ are Minkowski points and " $i \delta$ " means the Euclidean point $(\delta, 0, \ldots, 0)$. In our language it corresponds to the 4-point function $\left\langle\Psi\left(x_{1}\right) \mathcal{O}\left(x_{2}\right) \mathcal{O}\left(x_{3}\right) \Psi\left(x_{4}\right)\right\rangle$ with $\varepsilon_{1}=-\varepsilon_{4}=\delta>0$ and $\varepsilon_{2}=\varepsilon_{3}=0$. We know that the 4-point function is regular analytic at such configurations. So the commutator $[\mathcal{O}(x), \mathcal{O}(y)]$ vanishes in the shockwave background when $x$ and $y$ are spacelike separated.
} 
sum is absolutely convergent when $\left|\rho\left(c_{E}\right)\right|<1$ (see below). Also, when $d \geqslant 3$ we have $p_{\delta,-m}=p_{\delta, m}$, where $p_{\delta, m}=a_{12}(\delta, m) a_{\overline{43}}(\delta, m)^{*}$. Analogously to section 6.3 , the analytic continuation of $g_{1234}(c)$ in $d \geqslant 3$ will be given by the formula (compare (6.27))

$$
g_{1234}(c)=\left(\frac{x_{14}^{2} x_{23}^{2}}{x_{13}^{2} x_{24}^{2}}\right)^{\frac{\Delta_{1}-\Delta_{2}-\Delta_{3}+\Delta_{4}}{4}} \sum_{m, \delta, 0 \leqslant m \leqslant \delta} p_{\delta, m} R_{\delta / 2-m / 2}(c) \Phi_{m}(c) .
$$

In $d=2, p_{\delta, m} \neq p_{\delta,-m}$ but the functions $\rho(c)^{m}$ and $\bar{\rho}(c)^{m}$ are individually holomorphic. In this case the analytic continuation of $g_{1234}(c)$ is given by the formula (compare (6.31)):

$$
g_{1234}(c)=\left(\frac{x_{14}^{2} x_{23}^{2}}{x_{13}^{2} x_{24}^{2}}\right)^{\frac{\Delta_{1}-\Delta_{2}-\Delta_{3}+\Delta_{4}}{2}} \sum_{m, \delta, 0 \leqslant m \leqslant \delta} R_{\delta / 2-m / 2}(c)\left[p_{\delta, m} \rho(c)^{m}+p_{\delta,-m} \bar{\rho}(c)^{m}\right] .
$$

We would like to show that

(a) when $r=\max \{|\rho|,|\bar{\rho}|\}<1$, the series

$$
\tilde{g}_{1234}(\rho, \bar{\rho})=\sum_{\delta, m} a_{12}(\delta, m) a_{\overline{43}}(\delta, m)^{*} \rho^{(\delta+m) / 2} \bar{\rho}^{(\delta-m) / 2}
$$

is absolutely convergent;

(b) the remainder $\tilde{g}_{1234}\left(\rho, \bar{\rho} ; \delta_{*}\right):=\sum_{\delta \geqslant \delta_{*}, m} a_{12}(\delta, m) a_{\overline{43}}(\delta, m)^{*} \rho^{(\delta+m) / 2} \bar{\rho}^{(\delta-m) / 2}$ has a powerlaw bound, uniform in $\delta_{*}$ :

$$
\left|\tilde{g}_{1234}\left(\rho, \bar{\rho} ; \delta_{*}\right)\right| \leqslant C(1-r)^{-\Delta_{1}-\Delta_{2}-\Delta_{3}-\Delta_{4}} .
$$

This is done as follows (compare [36], section 4.2). Consider the 4-point functions $\left\langle\mathcal{O}_{1} \mathcal{O}_{2} \mathcal{O}_{2}^{\dagger} \mathcal{O}_{1}^{\dagger}\right\rangle,\left\langle\mathcal{O}_{4}^{\dagger} \mathcal{O}_{3}^{\dagger} \mathcal{O}_{3} \mathcal{O}_{4}\right\rangle$, and let $\tilde{g}_{12 \overline{21}}, \tilde{g}_{\overline{43} 34}$ be the analogues of (6.125):

$$
\begin{aligned}
& \tilde{g}_{12 \overline{21}}(\rho, \bar{\rho})=\sum_{\delta, m}\left|a_{12}(\delta, m)\right|^{2} \rho^{(\delta+m) / 2} \bar{\rho}^{(\delta-m) / 2} \\
& \tilde{g}_{\overline{4334}}(\rho, \bar{\rho})=\sum_{\delta, m}\left|a_{\overline{43}}(\delta, m)\right|^{2} \rho^{(\delta+m) / 2} \bar{\rho}^{(\delta-m) / 2}
\end{aligned}
$$

Noticing that $|m| \leqslant \delta$, we estimate (6.125) by absolute value and apply Cauchy-Schwarz inequality:

$$
\left|\tilde{g}_{1234}\left(\rho, \bar{\rho} ; \delta_{*}\right)\right| \leqslant \sum_{\delta, m}\left|a_{12}(\delta, m)\right|\left|a_{\overline{43}}(\delta, m)\right| r^{\delta} \leqslant\left[\tilde{g}_{12 \overline{21}}(r, r) \tilde{g}_{\overline{4334}}(r, r)\right]^{1 / 2} .
$$

The functions $\tilde{g}_{12 \overline{21}}(r, r)$ and $\tilde{g}_{\overline{4334}}(r, r)$ correspond to the 4-point functions at the Euclidean configurations with $\rho=\bar{\rho}=r<1$, hence their series expansions (6.127), (6.128) are convergent by the Euclidean OPE axiom. Therefore, (6.125) is absolutely convergent when $|\rho|,|\bar{\rho}|<1$. This finishes the proof of part (a).

Using the t-channel OPE, we can show that for $0 \leqslant r<1$,

$$
\begin{aligned}
& \tilde{g}_{12 \overline{21}}(r, r) \leqslant C(1-r)^{-2 \Delta_{1}-2 \Delta_{2}}, \\
& \tilde{g}_{\overline{43} 34}(r, r) \leqslant C(1-r)^{-2 \Delta_{3}-2 \Delta_{4}},
\end{aligned}
$$

with some $C>0$. Combining (6.130), (6.131) with (6.129) we get (6.126). This finishes the proof of part (b). 


\section{Optimal powerlaw bound from Cauchy-Schwarz $\rho, \bar{\rho}$ inequality}

In section 6.5 we provided a powerlaw bound for the 4-point function, based on the inequality (6.53) for $\max (|\rho(c)|,|\bar{\rho}(c)|)$. That did the job of allowing us to apply theorem 4.1 and prove that the Minkowski 4-point function is a distribution, but the actual bound (6.53) is not optimal. It is interesting to get a better bound on $|\rho(c)|,|\bar{\rho}(c)|$, because this will translate into a better powerlaw bound for the 4-point function, allowing us to get a better idea about the regularity of the Minkowski 4-point function as a distribution, i.e. how many derivatives test functions must have. In the proof of theorem 4.1, parameters $A_{n}$ and $B_{n}$ of the powerlaw bound enter into eq. (C.6) which provides an upper bound on the regularity.

In this section we will provide such an optimal bound on $|\rho(c)|,|\bar{\rho}(c)|$. The main idea of the bound and of its proof is inspired by section 6.7.2. Let us denote by $\mathcal{D}_{4}^{(0)}$ the subset of configurations $c \in \mathcal{D}_{4}$ satisfying the condition $\operatorname{Re} x_{1}^{0}>\operatorname{Re} x_{2}^{0}>0>\operatorname{Re} x_{3}^{0}>\operatorname{Re} x_{4}^{0}$. We showed that the 4-point functions for complexified times satisfy the Cauchy-Schwarz inequality (6.103) for $c=\left(x_{1}, x_{2}, x_{3}, x_{4}\right) \in \mathcal{D}_{4}^{(0)}$. For a general configuration $c \in \mathcal{D}_{4}^{(0)}$ we define two configurations

$$
c_{12}=\left(x_{1}, x_{2}, x_{2}^{\theta}, x_{1}^{\theta}\right), \quad c_{34}=\left(x_{4}^{\theta}, x_{3}^{\theta}, x_{3}, x_{4}\right),
$$

where $\theta$ is the operation in (6.91) which generalizes the OS reflection to complexified times. We will call such configurations, for obvious reasons, reflection-symmetric. It is clear that both $c_{12}, c_{34} \in \mathcal{D}_{4}^{(0)}$. Eq. (6.103) can now be written as

$$
\left|G_{4}(c)\right|^{2} \leqslant G_{4}\left(c_{12}\right) G_{4}\left(c_{34}\right) \quad\left(c \in \mathcal{D}_{4}^{(0)}\right) .
$$

Since we know that $G_{4}$ can be written as a convergent power series in $\rho, \bar{\rho}$, eq. (7.2) suggests that there should be a corresponding bound for the $\rho, \bar{\rho}$ coordinates. This is indeed the case, as we have the following couple of results:

Lemma 7.1. Any reflection-symmetric configuration $c \in \mathcal{D}_{4}^{(0)}$ has $\rho(c), \bar{\rho}(c) \in(0,1)$.

Theorem 7.2 (Cauchy-Schwarz inequality for $\rho, \bar{\rho}$ ). For any configuration $c \in \mathcal{D}_{4}^{(0)}$ we have the inequality:

$$
\max \{|\rho(c)|,|\bar{\rho}(c)|\}^{2} \leqslant \max \left\{\rho\left(c_{12}\right), \bar{\rho}\left(c_{12}\right)\right\} \times \max \left\{\rho\left(c_{34}\right), \bar{\rho}\left(c_{34}\right)\right\} .
$$

We will next prove lemma 7.1. We will then show how, combined with theorem 7.2, this implies an optimal bound on $\rho, \bar{\rho}$. Finally we will present a proof of theorem 7.2 , which is surprisingly subtle.

\subsection{Proof of lemma 7.1}

To prove the lemma, consider a reflection-symmetric configuration $c$ as in (7.1) with:

$$
\begin{array}{ll}
x_{1}=\left(\epsilon_{1}+i t_{1}, \mathbf{x}_{1}\right), & x_{2}=\left(\epsilon_{2}+i t_{2}, \mathbf{x}_{2}\right), \quad \epsilon_{1}>\epsilon_{2}>0, \\
x_{3}=x_{2}^{\theta}=\left(-\epsilon_{2}+i t_{2}, \mathbf{x}_{2}\right), & x_{4}=x_{1}^{\theta}=\left(-\epsilon_{1}+i t_{1}, \mathbf{x}_{1}\right) .
\end{array}
$$


We will compute $z(c), \bar{z}(c)$ explicitly. We can use translations in the $\mathbf{x}$ direction, as well as spatial rotations to simplify these computations. All these transformations do not change the conformal class of configuration, hence preserve $u, v$ and $z, \bar{z}$. They also commute with time reflection, and so map reflection-symmetric configurations to reflection-symmetric ones. By using this freedom, we get an equivalent configuration $c^{\prime}$ with the same $z, \bar{z}$ :

$$
x_{1}^{\prime}=\left(\epsilon_{1}+i t_{1}, \mathbf{0}\right), \quad x_{2}^{\prime}=\left(\epsilon_{2}+i t_{2},\left|\mathbf{x}_{2}-\mathbf{x}_{1}\right|, 0, \ldots, 0\right), \quad x_{3}^{\prime}=\left(x_{2}^{\prime}\right)^{\theta}, \quad x_{4}^{\prime}=\left(x_{1}^{\prime}\right)^{\theta} .
$$

This is an effectively two-dimensional configuration. The $z, \bar{z}$ variables of a two-dimensional 4-point configuration $x_{k}=\left(x_{k}^{0}, x_{k}^{1}\right)$ are given by eq. (6.30), which we copy here

$$
z=\frac{\left(z_{1}-z_{2}\right)\left(z_{3}-z_{4}\right)}{\left(z_{1}-z_{3}\right)\left(z_{2}-z_{4}\right)}, \quad \bar{z}=\frac{\left(\bar{z}_{1}-\bar{z}_{1}\right)\left(\bar{z}_{3}-\bar{z}_{4}\right)}{\left(\bar{z}_{1}-\bar{z}_{3}\right)\left(\bar{z}_{2}-\bar{z}_{4}\right)}, \quad z_{k}=x_{k}^{0}+i x_{k}^{1}, \quad \bar{z}_{k}=x_{k}^{0}-i x_{k}^{1} .
$$

Applying this to the configuration $c^{\prime}$, we get $z, \bar{z}$ for $c^{\prime}$ (which are the same as for $c$ ). It's easy to see that $z_{3}-z_{4}=\left(z_{1}-z_{2}\right)^{*}, z_{1}-z_{3}=\left(z_{2}-z_{4}\right)^{*}$ as a consequence of reflection symmetry, and similarly for $\bar{z}$ 's. So we get $z(c), \bar{z}(c)$ both real and positive. Explicit expressions come out to be

$$
\begin{aligned}
& z(c)=\frac{\left(\epsilon_{1}-\epsilon_{2}\right)^{2}+\left(t_{1}-t_{2}-\left|\mathbf{x}_{1}-\mathbf{x}_{2}\right|\right)^{2}}{\left(\epsilon_{1}+\epsilon_{2}\right)^{2}+\left(t_{1}-t_{2}-\left|\mathbf{x}_{1}-\mathbf{x}_{2}\right|\right)^{2}} \\
& \bar{z}(c)=\frac{\left(\epsilon_{1}-\epsilon_{2}\right)^{2}+\left(t_{1}-t_{2}+\left|\mathbf{x}_{1}-\mathbf{x}_{2}\right|\right)^{2}}{\left(\epsilon_{1}+\epsilon_{2}\right)^{2}+\left(t_{1}-t_{2}+\left|\mathbf{x}_{1}-\mathbf{x}_{2}\right|\right)^{2}} .
\end{aligned}
$$

In particular we see that $0<z(c), \bar{z}(c)<1$. The function $f(\zeta)$ in the definition of $\rho$ variables maps the interval $(0,1)$ to itself. Hence also $0<\rho(c), \bar{\rho}(c)<1$, and the lemma and proved.

\subsection{Optimal bound for $\rho, \bar{\rho}$}

We wish to derive a powerlaw bound on $\frac{1}{1-r}, r=\max (|\rho|,|\bar{\rho}|)$, since by the arguments in section 6.5 this implies a powerlaw bound for the 4-point function. Our aim here is to improve on (6.53), (6.54).

Consider first a configuration $c \in \mathcal{D}_{4}^{(0)}$. For such a configuration, by theorem 7.2, we have

$$
r(c) \leqslant \sqrt{r\left(c_{12}\right) r\left(c_{34}\right)} \leqslant \max \left(r\left(c_{12}\right), r\left(c_{34}\right)\right),
$$

and hence

$$
\frac{1}{1-r(c)} \leqslant \max \left(\frac{1}{1-r\left(c_{12}\right)}, \frac{1}{1-r\left(c_{34}\right)}\right) .
$$

We are thus reduced to study $r(c)$ for reflection-symmetric configurations, like in (7.4). By definition (6.9) of $\rho$ variables, we have

$$
\frac{1}{1-\rho}=\frac{1+\sqrt{1-z}}{2 \sqrt{1-z}} \leqslant \frac{1}{\sqrt{1-z}}, \quad z \in[0,1)
$$


so it suffices to study $1 /(1-z)$ and $1 /(1-\bar{z})$. Using $z, \bar{z}$ for reflection-symmetric configurations computed in eqs. (7.7) we have

$$
\frac{1}{1-z\left(c_{12}\right)}=\frac{\left(\epsilon_{1}+\epsilon_{2}\right)^{2}+\left(t_{1}-t_{2}-\left|\mathbf{x}_{1}-\mathbf{x}_{2}\right|\right)^{2}}{4 \epsilon_{1} \epsilon_{2}}
$$

and an analogous relation for $\frac{1}{1-\bar{z}\left(c_{12}\right)}$. From these equations, using $\epsilon_{2}<\epsilon_{1}$, and estimating $\epsilon_{1}-\epsilon_{2},\left|t_{1}-t_{2}\right|,\left|\mathbf{x}_{1}-\mathbf{x}_{2}\right|$ from above by $\left|x_{1}-x_{2}\right|$ (see (4.10)), we easily get

$$
\frac{1}{1-z\left(c_{12}\right)}, \frac{1}{1-\bar{z}\left(c_{12}\right)} \leqslant\left(1+\frac{1}{\epsilon_{2}}\right)^{2}\left(1+\left|x_{1}-x_{2}\right|\right)^{2},
$$

Putting together this relation, an analogous relation for $z\left(c_{34}\right), \bar{z}\left(c_{34}\right)$, eqs. (7.9) and (7.10), we get

$$
\frac{1}{1-r(c)} \leqslant \max \left\{\left(1+\frac{1}{\epsilon_{2}}\right)\left(1+\left|x_{1}-x_{2}\right|\right),\left(1+\frac{1}{\left|\epsilon_{3}\right|}\right)\left(1+\left|x_{3}-x_{4}\right|\right)\right\} \quad\left(c \in \mathcal{D}_{4}^{(0)}\right) .
$$

This was for $c \in \mathcal{D}_{4}^{(0)}$. For a general configuration $c \in \mathcal{D}_{4}$, we will shift the coordinates by a translation in time direction (which of course does not change $\rho, \bar{\rho}$ ), arranging so that the shifted configurations $c^{\prime}$ has $\epsilon_{2}>0>\epsilon_{3}$, i.e. $c^{\prime} \in \mathcal{D}_{4}^{(0)}$. Specifically we will choose

$$
\epsilon_{2}\left(c^{\prime}\right)=\frac{1}{2}\left(\epsilon_{2}(c)-\epsilon_{3}(c)\right), \quad \epsilon_{3}\left(c^{\prime}\right)=-\frac{1}{2}\left(\epsilon_{2}(c)-\epsilon_{3}(c)\right) .
$$

Then, using (7.13) for $c^{\prime}$, we obtain a bound on $\frac{1}{1-r(c)}$ which for example can be expressed as

$$
\frac{1}{1-r(c)} \leqslant 2\left(1+\frac{1}{\epsilon_{2}-\epsilon_{3}}\right)\left(1+\max \left\{\left|x_{1}-x_{2}\right|,\left|x_{3}-x_{4}\right|\right\}\right) \quad\left(c \in \mathcal{D}_{4}\right) .
$$

This is a powerlaw bound of the type we were looking for. By considering reflectionsymmetric configurations, it's easy to see that the exponents in this bound cannot be improved. Eq. (7.15) is much stronger than our previous suboptimal bound (6.53); in fact it implies a bound of the same form as (6.53) with the power exponent 12 replaced by 2 .

\subsection{Proof of theorem 7.2}

Although (7.3) looks like a simple-enough geometric inequality, we do not know an elementary proof of this fact. We essentially guessed this inequality, checked it numerically, and then looked for a proof. Our guess started in the Euclidean region, where $\bar{\rho}=\rho^{*}$, and (7.3) takes the form

$$
|\rho(c)|^{2} \leqslant \rho\left(c_{12}\right) \rho\left(c_{34}\right) \quad\left(c \in \mathcal{D}_{4}^{(0)} \quad \text { Euclidean }\right)
$$

Even in this case we don't know an elementary proof. We guessed that this must hold, because otherwise it was hard to imagine that the 4-point function itself would satisfy a Cauchy-Schwarz inequality. Indeed (7.16) implies the Euclidean version of (6.103). We then guessed (7.3) as a generalization of (7.16) for complexified times. 
Our proof of (7.3) reverses this logic, by deriving it from (6.103). There exist many explicit CFT 4-point functions, e.g. mean field theories (MFT). One could imagine that by considering (6.103) for a family of such 4-point functions, and passing to some limit (e.g. of scaling dimension of the mean field going to infinity), one could recover (7.3). We haven't managed to make this work using MFTs, but a closely related strategy does work. Namely we will apply this sort of argument not to the full 4-point function, but to a single conformal block, since the latter also satisfy (6.103) (as we will explain).

Now that we explained the main idea, let us supply the details. By applying a translation, we may set $\mathbf{x}_{1}=0$. The remaining spacial component vectors $\mathbf{x}_{2}, \mathbf{x}_{3}, \mathbf{x}_{4}$ span at most three-dimensional subspace of $\mathbb{R}^{d-1}$. This shows that it is enough to prove the inequality (7.3) in the case $d=4$. We assume that the readers are familiar with the conformal blocks, which encode contributions of a primary into a 4-point function. In the considered case of 4 identical Hermitean scalar, the relevant OPE has the form (simplifying the general case considered in section 3.1)

$$
\varphi\left(x_{1}\right) \varphi\left(x_{2}\right)=f_{\varphi \varphi \mathcal{O}} C_{(\lambda)}\left(x_{1}, x_{2}, x_{0}, \mathcal{D}_{0}\right) \mathcal{O}^{(\lambda)}\left(x_{0}\right)
$$

where $\mathcal{O}^{(\lambda)}$ is a dimension $\Delta$, spin $\ell$ symmetric traceless primary. The conformal block then can be computed by

$$
g_{\Delta, \ell}(c)=C_{(\lambda)}\left(x_{1}, x_{2}, x_{0}, \mathcal{D}_{0}\right) C_{(\mu)}\left(x_{3}^{\theta}, x_{4}^{\theta}, x_{0}^{\theta}, \mathcal{D}_{0}^{\theta}\right)\left\langle\mathcal{O}^{(\lambda)}\left(x_{0}\right) \mathcal{O}^{\dagger(\mu)}\left(x_{0}^{\theta}\right)\right\rangle .
$$

The 4d Euclidean conformal blocks are known explicitly [63, 64]:

$$
g_{\Delta, \ell}(c)=\frac{z \bar{z}}{z-\bar{z}}\left[k_{h}(z) k_{\bar{h}-1}(\bar{z})-k_{h}(\bar{z}) k_{\bar{h}-1}(z)\right]
$$

where $h, \bar{h}=(\Delta \pm \ell) / 2$, and $k_{\beta}(z)=z^{\beta}{ }_{2} F_{1}(\beta, \beta, 2 \beta ; z)$. (We only cite the result for external operators with equal dimensions.) We will assume that the exchanged operator $\mathcal{O}$ satisfies the $4 \mathrm{~d}$ unitarity bound $\Delta \geqslant \ell+2$. As eq. (7.19) shows, Euclidean conformal blocks are realanalytic functions whenever $|z|<1$. We can also use this formula to analytically continue them to the forward tube. We wish to show that this analytic continuation satisfies some properties. This is best shown not from the explicit formula, but by adapting the robust 4-point function arguments from section 6. Indeed, conformal blocks allow an expansion of the same form as (6.11), with non-negative coefficients which are fixed by conformal invariance. This can be shown by arguments similar to those in section 6.2. The existence of the representation (7.18) guarantees Hilbert space unitarity. Then, by the arguments of section 6.3, conformal blocks admit an analytic extension to the forward tube (which is of course the same as the one following from the explicit formula (7.19)). The point of the current construction is that it shows that the analytic extension satisfies an inequality analogous to $(6.46)$ :

$$
\left|g_{\Delta, \ell}(c)\right| \leqslant g_{\Delta, \ell}\left(c_{*}\right)
$$

Then, by the arguments in section 6.5, conformal blocks satisfy the powerlaw bound in the forward tube. (As is easy to see from (7.19), 4d Euclidean conformal blocks grow 
as $1 /(1-z)$ as $z \rightarrow 1^{-}$along the real axis, which replaces eq. (6.48).) Finally, by the arguments analogous to section 6.7.2 we conclude that the analytically continued conformal blocks satisfy Cauchy-Schwarz inequality:

$$
\left|g_{\Delta, l}(c)\right|^{2} \leqslant g_{\Delta, l}\left(c_{12}\right) g_{\Delta, l}\left(c_{34}\right) \text { for any } c \in \mathcal{D}_{4}^{(0)} .
$$

(Euclidean reflection positivity of conformal blocks follows from the representation (7.18), which we assume to be valid in the Euclidean region.)

In the rest of the argument we will only need two facts, the Cauchy-Schwarz inequality (7.21) and the explicit Dolan-Osborn formula (7.19). We will apply (7.21) to the blocks of spin $\ell \geqslant 1$ at the unitarity bound, i.e. with $\bar{h}=1, h=\ell+1$. The Cauchy-Schwarz inequality for $\rho, \bar{\rho}$ will follow by extracting the asymptotics in the limit $h \rightarrow+\infty$. The asymptotic behavior of $k_{h}$ is given by the following lemma:

Lemma 7.3. For any fixed $z \in \mathbb{C} \backslash[1,+\infty)$, the function $k_{h}(z)$ has the following asymptotic behavior in terms of the $\rho$ variable defined in (6.9):

$$
k_{h}(z)=(4 \rho)^{h}\left[\frac{1}{\sqrt{1-\rho^{2}}}+o(1)\right], \quad h \rightarrow+\infty .
$$

Proof. We have the following identity for $k_{h}(z)[66]$ :

$$
k_{h}(z)=(4 \rho)_{2}^{h} F_{1}\left(1 / 2, h ; h+1 / 2 ; \rho^{2}\right) .
$$

The region $z \notin[1,+\infty)$ corresponds to $|\rho|<1$, where the hypergeometric function ${ }_{2} F_{1}$ has the power series representation

$$
{ }_{2} F_{1}\left(1 / 2, h ; h+1 / 2 ; \rho^{2}\right)=\sum_{n=0}^{\infty} \frac{(1 / 2)_{n}(h)_{n}}{n !(h+1 / 2)_{n}} \rho^{2 n} .
$$

When $h \rightarrow+\infty$, each coefficient of the series increases monotonically, and tends to the coefficients of the convergent in the disk $|\rho|<1$ series

$$
\sum_{n=0}^{\infty} \frac{(1 / 2)_{n}}{n !} \rho^{2 n}=\frac{1}{\sqrt{1-\rho^{2}}}
$$

This implies the statement of the lemma.

Consider now inequality (7.21) for the blocks with $\bar{h}=1, h=\ell+1$. Since $k_{0} \equiv 1$, it reads:

$$
\left|w \cdot\left[k_{h}(z)-k_{h}(\bar{z})\right]\right|^{2} \leqslant w_{12} w_{34} \cdot\left[k_{h}\left(z_{12}\right)-k_{h}\left(\bar{z}_{12}\right)\right]\left[k_{h}\left(z_{34}\right)-k_{h}\left(\bar{z}_{34}\right)\right],
$$

where we denoted $w=\frac{z \bar{z}}{z-\bar{z}}$, and similarly $w_{12}, w_{34}$. Let us assume that the configuration $c \in \mathcal{D}_{4}^{(0)}$ is such that

$$
|\rho| \neq|\bar{\rho}|, \quad \rho_{12} \neq \bar{\rho}_{12}, \quad \rho_{34} \neq \bar{\rho}_{34} .
$$


Then, using lemma 7.3, for large $h$ inequality (7.25) becomes:

$$
(A+o(1)) \max \{|\rho|,|\bar{\rho}|\}^{2 h} \leqslant(B+o(1)) \max \left\{\rho_{12}, \bar{\rho}_{12}\right\}^{h} \max \left\{\rho_{34}, \bar{\rho}_{34}\right\}^{h},
$$

where $A, B$ are some positive $h$-independent quantities. Now, taking the limit $h \rightarrow+\infty$, we obtain inequality (7.3).

It's easy to see that configurations which violate the condition (7.26) are non-generic. They can be approached by configurations which do satisfy (7.26). Therefore, by continuity inequality (7.3) is valid also for such exceptional configurations.

\section{OPE convergence in the forward tube and in Minkowski space}

We have several OPE convergence statements scattered throughout the paper. The Euclidean CFT axioms assume convergence of the OPE series for $\mathcal{O}_{1}\left(x_{1}\right) \mathcal{O}_{2}\left(x_{2}\right)$ whenever the two points $x_{1}, x_{2}$ are closer to the OPE center than any other point. Then we established OPE convergence in the Hilbert space sense (section 3.2) in the Euclidean region for states generated by two operators in the half-space. Then in section 6.2 we used Hilbert space language to derive the power series representation (6.11) for the 4-point function, whose convergence is thus morally equivalent to OPE convergence (for the 4-point functions). We then used this power series representation to analytically continue the 4-point function to the forward tube, and then define the Minkowski 4-point function as a boundary value in the sense of distributions. Finally, in section 6.7 we showed, by arguments not using conformal invariance, that the OS states $\left|\mathcal{O}_{1}\left(x_{1}\right) \mathcal{O}_{2}\left(x_{2}\right)\right\rangle$ can be extended to the forward tube and (when integrated against test functions) to the Minkowski region, and that the so obtained states can be arbitrarily well approximated by (integrated) OS states. Therefore, OPE convergence holds for these states, as for the OS states.

In this section we will give a more explicit discussion of the OPE convergence for the Minkowski 4-point function and for the 2-operator states in the forward tube and Minkowski space. We will also explain how our approach and results compare to the classic paper by Mack [41].

\subsection{Convergence of conformal block decomposition for 4-point functions}

Let us consider the 4-point function of identical scalars (6.1):

$$
G\left(x_{1}, x_{2}, x_{3}, x_{4}\right) \equiv G(c)=\left\langle\mathcal{O}\left(x_{1}\right) \mathcal{O}\left(x_{2}\right) \mathcal{O}\left(x_{3}\right) \mathcal{O}\left(x_{4}\right)\right\rangle=\frac{g(\rho, \bar{\rho})}{\left(x_{12}^{2} x_{34}^{2}\right)^{\Delta_{\mathcal{O}}}}
$$

The discussion below can be easily extended to non-identical scalars using the same ideas as in section 6.10 .

We know that in the Euclidean region the function $g(\rho, \bar{\rho})$ has a convergent conformal block decomposition

$$
g(\rho, \bar{\rho})=\sum_{\Delta, l} C_{\Delta, l}^{2} g_{\Delta, l}(\rho, \bar{\rho})
$$


As in section 7.3, we will assume that the reader is familiar with conformal blocks. The main point is that the conformal block decomposition is obtained by separating the series (6.11) into parts corresponding to the conformal multiplets of primary operators $\mathcal{O}_{\Delta, l}$ occurring in the $\mathcal{O} \times \mathcal{O}$ OPE with coefficients $C_{\Delta, l}$. Conformal blocks in the Euclidean region by themselves have power series expansions like (6.11) with positive coefficients (fixed by conformal symmetry). As in section 6.3 , we can use this expansion to analytically continue conformal blocks to the forward tube. By an analogue of the bound (6.28) we know that conformal block expansion remains convergent everywhere in the forward tube, since $|\rho|,|\bar{\rho}|<1$ there. Since individual conformal blocks are smaller than the 4-point function in the Euclidean region, by the arguments in section 6.5 we know that they satisfy a powerlaw bound, and hence they become tempered distributions when going to the Minkowski region. ${ }^{65,66,67}$

By the arguments like in section $6.5, g(\rho, \bar{\rho})$, the partial sums of the conformal block decomposition $g\left(\rho, \bar{\rho} ; \Delta_{*}\right)=\sum_{\Delta \leqslant \Delta_{*}, l} C_{\Delta, l}^{2} g_{\Delta, l}(\rho, \bar{\rho})$, and the corresponding remainders satisfy in the forward tube a uniform bound:

$$
\left|g\left(\rho, \bar{\rho} ; \Delta_{*}\right)\right|,\left|g(\rho, \bar{\rho})-g\left(\rho, \bar{\rho} ; \Delta_{*}\right)\right| \leqslant C(1-r)^{-4 \Delta}, \quad r=\max \{|\rho|,|\bar{\rho}|\} .
$$

Consider the 4-point partial sums including the prefactor $G\left(c ; \Delta_{*}\right)=\frac{1}{\left(x_{12}^{2} x_{34}^{2}\right)^{\Delta_{\mathcal{O}}}} g\left(\rho, \bar{\rho} ; \Delta_{*}\right)$. By the powerlaw bound of $(1-r(c))^{-1}$, we have the powerlaw bounds

$$
\left|G\left(c ; \Delta_{*}\right)\right|,\left|G(c)-G\left(c ; \Delta_{*}\right)\right| \leqslant C\left(1+\max _{k} \frac{1}{\epsilon_{k}-\epsilon_{k+1}}\right)^{A}\left(1+\max _{i}\left|x_{i}-x_{i+1}\right|\right)^{B}
$$

for all $c \in \mathcal{D}_{4}$ and $\Delta_{*} \geqslant 0$. Consider the boundary value of $G\left(c ; \Delta_{*}\right)$, call it $G^{M}\left(x_{1}, x_{2}, x_{3}, x_{4} ; \Delta_{*}\right)$, where $x_{i} \in \mathbb{R}^{1, d-1} ;$ it is a tempered distribution by theorem 4.1. The following theorem establishes distributional convergence of conformal block decomposition.

Theorem 8.1. We have $G^{M}\left(x ; \Delta_{*}\right) \rightarrow G^{M}(x)$ in the sense of tempered distributions.

Proof. Denote $H\left(c ; \Delta_{*}\right)=G(c)-G\left(c ; \Delta_{*}\right)$. We have to show that, as $\Delta_{*}$ goes to infinity, the boundary value of $H\left(c ; \Delta_{*}\right)$ converges to 0 in the sense of tempered distributions, i.e,

\footnotetext{
${ }^{65}$ This argument shows that any conformal block which occurs in a reflection-positive CFT 4-point function satisfies a powerlaw bound. E.g. conformal blocks for $l \geqslant 0, \Delta \geqslant l+d-2$ occur in a 4-point function $\left\langle\varphi_{1} \varphi_{2} \varphi_{1} \varphi_{2}\right\rangle$ where $\varphi_{1}, \varphi_{2}$ are two GFFs of appropriately chosen equal dimension. It should be also possible to show that conformal blocks satisfy a powerlaw bound without relying on a fiducial 4-point function. E.g. for $d=4$ conformal blocks this follows from their explicit Dolan-Osborn expressions. For general $d$, powerlaw bound on the diagonal $z=\bar{z}$ can be shown using the differential equation found in [78] and extended to $z \neq \bar{z}$ by the usual arguments.

${ }^{66}$ It should be noted that away from light cones conformal blocks are better than distributions: they are real-analytic there (although this fact won't play a role for us). In even $d$ this is obvious from their explicit expressions in terms of hypergeometric functions. For general $d$ this follows from a first-order matrix ODE satisfied by a finite-length vector including the conformal block and its low-order derivatives. Such an ODE exists for a length-8 vector and can be built using the quadratic and quartic Casimir equations [79].

${ }^{67}$ Also "conformal partial waves" $\frac{g_{\Delta, l}(\rho, \bar{\rho})}{\left(x_{12}^{2} x_{34}^{2}\right)^{\Delta} \mathcal{O}}$ are tempered distributions in the Minkowski space. Therefore their Fourier transforms are well defined. Explicit expressions for these Fourier transforms were found recently in $[80]$.
} 
for any Schwartz test function $f \in \mathcal{S}\left(\mathbb{R}^{4 d}\right)$

$$
\lim _{\Delta_{*} \rightarrow \infty} \lim _{\lambda \rightarrow 0^{+}} \int H\left(\lambda \epsilon+i t, \mathbf{x} ; \Delta_{*}\right) f(t, \mathbf{x}) d t d \mathbf{x}=0, \quad\left(\epsilon_{1}>\epsilon_{2}>\epsilon_{3}>\epsilon_{4}\right),
$$

where we write for brevity $t$ instead of $t_{1}, t_{2}, t_{3}, t_{4}$ etc. The proof is the same as in our paper [36], theorem 3.1. We will retrace here the main steps for completeness and because we will need it to establish a stronger result below. Define $L_{f}\left(\lambda ; \Delta_{*}\right):=\int H\left(\lambda \epsilon+i t, \mathbf{x} ; \Delta_{*}\right) f(x) d x$ with $x=(t, \mathbf{x})$. Since $H$ is holomorphic in $\tau=\lambda \epsilon+i t$ we have

$$
\begin{aligned}
L_{f}^{(n)}\left(\lambda ; \Delta_{*}\right) & =\int\left(\left(\epsilon \cdot \frac{\partial}{i \partial t}\right)^{n} H\left(\lambda \epsilon+i t, \mathbf{x} ; \Delta_{*}\right)\right) f(x) d x \\
& =\int H\left(\lambda \epsilon+i t, \mathbf{x} ; \Delta_{*}\right)\left(\left(i \epsilon \cdot \frac{\partial}{\partial t}\right)^{n} f(x)\right) d x
\end{aligned}
$$

which by the powerlaw bound (8.4) implies

$$
L_{f}^{(n)}\left(\lambda ; \Delta_{*}\right) \leqslant \frac{C_{n}}{\lambda^{A}}|f|_{p_{n}}, \quad \lambda \in(0,1], \quad p_{n}=\max \{n,\lceil B\rceil+4 d+1\} .
$$

These bounds blow up in the $\lambda \rightarrow 0$ limit, but by using the Newton-Leibniz repeatedly one can get bounds which do not blow up:

$$
L_{f}^{(n)}\left(\lambda ; \Delta_{*}\right) \leqslant D_{n}|f|_{p_{n+[A]+1}}, \quad \lambda \in(0,1] .
$$

Using this for $n=1$ one proves that the limit $L_{f}\left(0 ; \Delta_{*}\right)=\lim _{\lambda \rightarrow 0^{+}} L_{f}\left(\lambda ; \Delta_{*}\right)$ exists and

$$
\left|L_{f}\left(0 ; \Delta_{*}\right)-L_{f}\left(\lambda ; \Delta_{*}\right)\right| \leqslant D_{1} \lambda|f|_{\max \{[A]+2,\lceil B\rceil+4 d+1\}} .
$$

By Lebesgue's dominated convergence theorem, for any fixed $\lambda$ in $(0,1], L_{f}\left(\lambda ; \Delta_{*}\right)$ tends to zero as $\Delta_{*} \rightarrow+\infty$. Thus the previous bound implies

$$
\begin{aligned}
\varlimsup_{\Delta_{*} \rightarrow \infty}\left|L_{f}\left(0, \Delta_{*}\right)\right| & \leqslant \varlimsup_{\Delta_{*} \rightarrow \infty}\left|L_{f}\left(0, \Delta_{*}\right)-L_{f}\left(\lambda ; \Delta_{*}\right)\right|+\varlimsup_{\Delta_{*} \rightarrow \infty}\left|L_{f}\left(\lambda ; \Delta_{*}\right)\right| \\
& \leqslant D_{1} \lambda|f|_{\max \{[A]+2,[B\rceil+4 d+1\} .}
\end{aligned}
$$

Since $\lambda$ can be arbitrarily small, we get $\varlimsup_{\Delta_{*} \rightarrow \infty}\left|L_{f}\left(0, \Delta_{*}\right)\right|=0$. This finishes the proof.

\subsubsection{Convergence rate for compactly supported test functions}

Because of the use of Lebesgue's theorem on dominated convergence, theorem 8.1 does not give the rate of convergence. We will now give the rate in an important special case of compactly supported test functions. This provides an explicit example for the remark in [36], the last paragraph of section 3.3.

The idea is that not only $H\left(c, \Delta_{*}\right) \rightarrow 0$ pointwise but it does so exponentially fast. We will first derive the exponential convergence bound, upgrading the Euclidean argument from [65], to the forward tube, and then use it. Let $F(t)$ be the Laplace transform of a positive measure $\mu(E)$ on $E \geqslant 0$ :

$$
F(t)=\int_{0}^{\infty} \mu(E) e^{-E t} d E, \quad \mu(E) \geqslant 0 .
$$


We assume that the integral is convergent for $t>0$ and $F(t) \sim t^{-\alpha}$ as $t \rightarrow 0^{+}$, and $\alpha>0$. Then by the Hardy-Littlewood tauberian theorem we know that

$$
M(E)=\int_{0}^{E} \mu\left(E^{\prime}\right) d E^{\prime} \sim \frac{E^{\alpha}}{\Gamma(\alpha+1)} \text { as } E \rightarrow+\infty .
$$

We can now estimate the remainder $F_{E_{*}}(t):=\int_{E_{*}}^{\infty} \mu(E) e^{-E t} d E$, via

$$
F_{E_{*}}(t)=\int_{E_{*}}^{\infty} e^{-E t} d M(E)=-M\left(E_{*}\right) e^{-E_{*} t}+t \int_{E_{*}}^{\infty} M(E) e^{-E t} d E,
$$

which gives $\left|F_{E_{*}}(t)\right| \leqslant C_{1} e^{-E_{*} t} E_{*}^{\alpha}+C_{2} e^{-E_{*} t}\left(\frac{1+E_{*} t}{t}\right)^{\alpha}$, and finally

$$
\left|F_{E_{*}}(t)\right| \leqslant \text { const } \times e^{-E_{*} t}\left(t^{-1}+E_{*}\right)^{\alpha} .
$$

for any $E_{*} \geqslant 1$ (say).

Now let's go back to the 4-point function of four identical scalars $G(c)=$ $\left\langle\mathcal{O}\left(x_{1}\right) \mathcal{O}\left(x_{2}\right) \mathcal{O}\left(x_{3}\right) \mathcal{O}\left(x_{4}\right)\right\rangle, c \in \mathcal{D}_{4}$. The remainder $H\left(c ; \Delta_{*}\right)$ can clearly be bounded by replacing $\rho, \bar{\rho}$ with $r=\max \{|\rho|,|\bar{\rho}|\}$ :

$$
\left|H\left(c ; \Delta_{*}\right)\right| \leqslant \frac{1}{\left|x_{12}^{2}\right|^{\Delta_{\mathcal{O}}}\left|x_{34}^{2}\right|^{\Delta_{\mathcal{O}}}}\left[g(r, r)-g\left(r, r ; \Delta_{*}\right)\right],
$$

By setting $t=\log (1 / r), g(r, r)$ with its representation (6.11) is in the same form as (8.11) and by eq. (6.48) we have that the corresponding $F(t) \sim t^{-\alpha}$ with $\alpha=4 \Delta_{\mathcal{O}}$. Bounding the remainder $g(r, r)-g\left(r, r ; \Delta_{*}\right)$ by (8.14), and using (8.15), we get a bound on the remainder $\left|H\left(c ; \Delta_{*}\right)\right|$ for any $\Delta_{*} \geqslant 1$ (say):

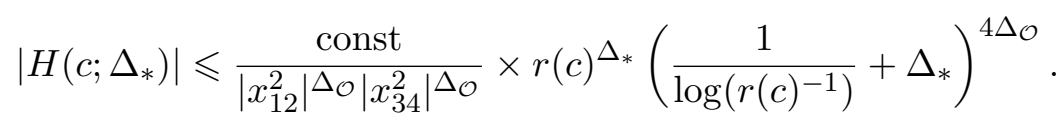

Let us now convert this into an explicit estimate for the distributional convergence rate, improving on theorem 8.1 for compactly supported test functions. Recall that we have an upper bound on $r(c)=\max \{|\rho(c)|,|\bar{\rho}(c)|\}\left(c=\left(x_{1}, x_{2}, x_{3}, x_{4}\right)\right)$, eq. (7.15), which we copy here:

$$
\frac{1}{1-r(c)} \leqslant 2\left(1+\frac{1}{\epsilon_{2}-\epsilon_{3}}\right)\left(1+\max \left\{\left|x_{1}-x_{2}\right|,\left|x_{3}-x_{4}\right|\right\}\right) .
$$

This bound tells us how much $r(c)$ is separated from 1. In turn, by (8.16) this translates into an explicit bound on $\left|H\left(c ; \Delta_{*}\right)\right|$. Let us retrace the proof of theorem 8.1, replacing eq. (8.10) by

$$
\begin{aligned}
\left|L_{f}\left(0, \Delta_{*}\right)\right| & \leqslant\left|L_{f}\left(0, \Delta_{*}\right)-L_{f}\left(\lambda ; \Delta_{*}\right)\right|+\left|L_{f}\left(\lambda ; \Delta_{*}\right)\right| \\
& \leqslant D_{1} \lambda|f|_{\max \{[A]+2,\lceil B\rceil+4 d+1\}}+\left|L_{f}\left(\lambda ; \Delta_{*}\right)\right| .
\end{aligned}
$$

We will now choose $\lambda$ small, as a function of $\Delta_{*}$, so that the second term in the r.h.s. is smaller than the first one. Let us choose and fix $\epsilon_{1}>\epsilon_{2}>\epsilon_{3}>\epsilon_{4}$. For $x_{k}^{\lambda}=\left(\lambda \epsilon_{k}+i t_{k}, \mathbf{x}_{k}\right)$, $x_{k}^{M}=\left(i t_{k}, \mathbf{x}_{k}\right)$, bound (8.17) gives

$$
\frac{1}{1-r\left(c^{\lambda}\right)} \leqslant \frac{C_{\epsilon}}{\lambda}\left(1+\max \left\{\left|x_{1}^{M}-x_{2}^{M}\right|,\left|x_{3}^{M}-x_{4}^{M}\right|\right\}\right) \quad(0<\lambda \leqslant 1),
$$


where $C_{\epsilon}$ is a constant which depends only on $\epsilon_{i}$ but not on $\lambda$ or $x_{k}^{M}$. If $f \in C_{0}^{\infty}\left(\mathbb{R}^{4 d}\right)$, a compactly supported test function, then (8.19) implies

$$
\frac{1}{1-r\left(c^{\lambda}\right)} \leqslant \frac{A_{f}}{\lambda} \quad\left(0<\lambda \leqslant 1,\left(x_{k}^{M}\right) \in \operatorname{supp}(f)\right),
$$

where $A_{f}=2 C_{\epsilon} \sup _{\left(x_{k}^{M}\right) \in \operatorname{supp}(f)}\left(1+\max \left\{\left|x_{1}^{M}-x_{2}^{M}\right|,\left|x_{3}^{M}-x_{4}^{M}\right|\right\}\right)$.

Now by (8.16), (8.20), and lemma 5.2(b) we have a bound for a compactly supported test function $f$ :

$$
\left|L_{f}\left(\lambda ; \Delta_{*}\right)\right| \leqslant \frac{\text { const }}{\lambda^{4 \Delta_{\mathcal{O}}}} e^{-\frac{\lambda}{A_{f}} \Delta_{*}}\left(\frac{A_{f}}{\lambda}+\Delta_{*}\right)^{4 \Delta_{\mathcal{O}}} \int|f| d x .
$$

By this and (8.18) we have

$$
\left|L_{f}\left(0 ; \Delta_{*}\right)\right| \leqslant A_{1} \lambda+\frac{A_{2}}{\lambda^{4 \Delta_{\mathcal{O}}}} e^{-\frac{\lambda}{A_{f}} \Delta_{*}}\left(\frac{A_{f}}{\lambda}+\Delta_{*}\right)^{4 \Delta_{\mathcal{O}}},
$$

where all constants $A_{1}, A_{2}, A_{f}$ are $f$-dependent. If we choose $\lambda=1 / \Delta_{*}^{\gamma}$ then for any $\gamma \in(0,1)$ the first term dominates (the second term decays exponentially fast for large $\Delta_{*}$ ). It is easy to see that the first term still dominates for $\lambda=\kappa \frac{\log \Delta_{*}}{\Delta_{*}}$ with sufficiently large $\kappa$. We therefore obtain the following promised strengthening of theorem 8.1.

Theorem 8.2. For any compactly supported $C^{\infty}$ test function $f$, we have $\left(G^{M}\left(\Delta_{*}\right), f\right)-$ $\left(G^{M}, f\right) \rightarrow 0$ as $O\left(\frac{\log \Delta_{*}}{\Delta_{*}}\right)$.

It is somewhat surprising that conformal block decomposition converges so slowly in the Minkowski region, while it converges exponentially fast in the Euclidean region.

\subsection{OPE convergence in the sense of $\mathcal{H}^{\mathrm{CFT}}$}

We will now rephrase the question of OPE convergence from the point of view of states generated by two operators (rather than 4-point functions which represent inner products of such states). We already discussed these questions to some extent in section 3.2 in the Euclidean region, and section 6.7 in the forward tube and in the Minkowski region. We will now update that discussion.

Recall that in section 6.7 we defined states (see eq. (6.88) and remark 6.3)

$$
\psi\left(x_{1}, x_{2}\right)=\left|\mathcal{O}\left(x_{1}\right) \mathcal{O}\left(x_{2}\right)\right\rangle, \quad x_{k}=\left(\epsilon_{k}+i t_{k}, \mathbf{x}_{k}+i \mathbf{y}_{k}\right), \quad 0 \succ\left(\epsilon_{1}, \mathbf{y}_{2}\right) \succ\left(\epsilon_{1}, \mathbf{y}_{2}\right),
$$

as elements of a vector space with inner products (6.90):

$$
\left\langle\mathcal{O}\left(x_{1}\right) \mathcal{O}\left(x_{2}\right) \mid \mathcal{O}\left(x_{3}\right) \mathcal{O}\left(x_{4}\right)\right\rangle=G_{4}\left(x_{2}^{\theta}, x_{1}^{\theta}, x_{3}, x_{4}\right),
$$

where $x^{\theta}=(-\epsilon+i t, \mathbf{x}-i \mathbf{y})$ for $x=(\epsilon+i t, \mathbf{x}+i \mathbf{y})$. We then proved that this inner product was positive definite, and that these states could be approximated in norm by Euclidean states, and so belong to the same Hilbert space. The arguments of section 6.7 did not use conformal symmetry. 
So, by arguments of section 6.7 we have a map $\psi\left(x_{1}, x_{2}\right)$ from $x_{1}, x_{2}$ as in (8.23) into $\mathcal{H}^{\mathrm{CFT}}$. We claim that this map is holomorphic. To begin with, this map is continuous with respect to the $\mathcal{H}^{\mathrm{CFT}}$ norm, and in particular bounded on compact subsets. This follows easily from the continuity of $G_{4}$. To show holomorphicity, we will use Morera's theorem and Osgood's lemma (which remain valid for Hilbert-space-valued functions of complex variables). Morera's theorem says that a locally continuous function of one complex variable is holomorphic if its integral over any small contour is zero. Let $C$ be a small $1 \mathrm{~d}$ contour in the region of $\xi=\left(x_{1}, x_{2}\right)$ where $\psi$ is defined (we assume that one of the components of $\xi$ goes around the contour while the others stay fix). We have

$$
\left\|\int_{C} d \xi \psi(\xi)\right\|^{2}=\int_{\xi^{\prime} \in C} d \overline{\xi^{\prime}} \int_{\xi \in C} d \xi G_{4}\left(\xi^{\prime \theta}, \xi\right)=0
$$

the last integral being zero because $G_{4}$ is holomorphic in $\xi$. Hence $\int_{C} d \xi \psi(\xi)=0$ and by Morera's theorem $\psi\left(x_{1}, x_{2}\right)$ is holomorphic in each component separately. Finally, by Osgood's lemma [68] $\psi\left(x_{1}, x_{2}\right)$ is holomorphic in all variables jointly.

Let us connect this discussion with the OPE. In the Euclidean region, OPE says

$$
\begin{gathered}
\psi\left(x_{1}, x_{2}\right)=\left|\mathcal{O}\left(x_{1}\right) \mathcal{O}\left(x_{2}\right)\right\rangle=\sum_{k, \lambda} f_{\mathcal{O O} k} C_{k, \lambda}\left(x_{1}, x_{2}, x_{S}, \mathcal{D}\right)\left|\left(\mathcal{O}_{k}^{\dagger}\right)^{(\lambda)}\left(x_{S}\right)\right\rangle, \\
C_{k, \lambda}\left(x_{1}, x_{2}, x_{S}, \mathcal{D}\right)=\sum_{\alpha} f_{\mathcal{O O} k} C_{k, \lambda, \alpha}\left(x_{1}, x_{2}, x_{S}\right) \mathcal{D}^{\alpha}
\end{gathered}
$$

where $x_{1}, x_{2}$ are two Euclidean points in the lower halfspace, $0>x_{1}^{0}, x_{2}^{0}, x_{S}=(-1,0, \ldots)$ is the south pole, and $\mathcal{D}=\mathcal{D}_{x_{S}}$ is the image of $\partial /\left.\partial x\right|_{x=0}$ under a conformal transformation which maps $0, \infty$ to $x_{S}, x_{N}$. We proved in section 3.2, from the Euclidean CFT axioms, that the series in the r.h.s. converges in $\mathcal{H}^{\mathrm{CFT}}$. As discussed in section 3.2, convergence holds provided that the series is summed in a certain manner: for each $\Lambda$ we define a partial sum $\psi_{\Lambda}\left(x_{1}, x_{2}\right)$ over all terms with $\Delta_{k}+|\alpha|<\Lambda$, and then tend $\Lambda \rightarrow \infty$. This procedure is needed because, although the states $\mathcal{D}^{\alpha}\left|\left(\mathcal{O}_{k}^{\dagger}\right)^{(\lambda)}\left(x_{S}\right)\right\rangle$ are orthogonal for different $|\alpha|$ (because they are eigenvectors of $\frac{K^{0}-P^{0}}{2}$ with different eigenvalues), they are not orthonormal. This can be corrected as follows. For each $k$, let us orthonormalize the infinite multiplet of states $\mathcal{D}^{\alpha}\left|\left(\mathcal{O}_{k}^{\dagger}\right)^{(\lambda)}\left(x_{S}\right)\right\rangle$. Let $e_{k, n}, n \in \mathbb{Z}_{\geqslant 0}$, be the corresponding orthonormal basis of states (there is obviously a lot of arbitrariness in this basis). We then can write

$$
\psi\left(x_{1}, x_{2}\right)=\left|\mathcal{O}\left(x_{1}\right) \mathcal{O}\left(x_{2}\right)\right\rangle=\sum_{k} f_{\mathcal{O O} k} \sum_{n} \tilde{C}_{k, n}\left(x_{1}, x_{2}\right) e_{k, n}
$$

where $\tilde{C}_{k, n}$ 's are some finite linear combinations of $C_{k, \lambda, \alpha}\left(x_{1}, x_{2}, x_{S}\right)$. Since $e_{k, n}$ 's with different $k$ are also orthogonal, this equation is an expansion of the state $\psi\left(x_{1}, x_{2}\right)$ in an orthonormal basis. Convergence of the series is now equivalent to the finiteness of the norm of $\psi\left(x_{1}, x_{2}\right)$ :

$$
\left\|\psi\left(x_{1}, x_{2}\right)\right\|_{\mathcal{H}^{\mathrm{CFT}}}=\sum_{k, n}\left|f_{\mathcal{O O O} k} \tilde{C}_{k, n}\left(x_{1}, x_{2}\right)\right|^{2}<\infty .
$$

Moreover, by definition, this norm is nothing but the 4-point function $\left\langle\mathcal{O}\left(x_{2}^{\theta}\right) \mathcal{O}\left(x_{1}^{\theta}\right) \mathcal{O}\left(x_{1}\right) \mathcal{O}\left(x_{2}\right)\right\rangle$. 
Eqs. (8.26)-(8.28) were all in the Euclidean region, but we claim that they continue to make sense in the forward tube. The argument is as follows. We know by the arguments around (8.25) that $\psi\left(x_{1}, x_{2}\right)$ have analytic continuation to the region (8.23). The inner product $\left\langle e_{k, n} \mid \psi\left(x_{1}, x_{2}\right)\right\rangle$ is then the analytic continuation of $f_{\mathcal{O O} k} \tilde{C}_{k, n}\left(x_{1}, x_{2}\right)$ from the Euclidean to the same region. (This inner product is a finite linear combination of $x_{N^{-}}$ derivatives of the 3-point function $\left\langle\left(\mathcal{O}_{k}\right)^{(\lambda)}\left(x_{N}\right) \mathcal{O}\left(x_{1}\right) \mathcal{O}\left(x_{2}\right)\right\rangle$, hence holomorphic in the forward tube.) We thus obtain the following fact:

Theorem 8.3. Expansion (8.27), analytically continued from the Euclidean region to the forward tube term by term, converges in the sense of $\mathcal{H}^{\mathrm{CFT}}$ to the same states $\psi\left(x_{1}, x_{2}\right)$ in the region (8.23) that we defined in section 6.7.

For the subsequent discussion, we also define the states

$$
\psi_{k}\left(x_{1}, x_{2}\right)=f_{\mathcal{O O} k} \sum_{n} \tilde{C}_{k, n}\left(x_{1}, x_{2}\right) e_{k, n}
$$

The norms of these states is given by conformal blocks (up to prefactor $f_{\mathcal{O O} k}^{2} /\left(x_{12}^{2}\right)^{4 \Delta_{\mathcal{O}}}$ ). Just as the state $\psi\left(x_{1}, x_{2}\right)$, each state $\psi_{k}\left(x_{1}, x_{2}\right)$ is an $\mathcal{H}^{\mathrm{CFT}}$-valued holomorphic function in the region (8.23), moreover in this region we have

$$
\psi\left(x_{1}, x_{2}\right)=\sum_{k} \psi_{k}\left(x_{1}, x_{2}\right)
$$

the series convergent in the sense of $\mathcal{H}^{\mathrm{CFT}}$. The norm of the tail of this series is given by the function $H\left(c, \Delta_{*}\right)$ from the proof of theorem 8.1:

$$
\left\|\sum_{\Delta_{k}>\Delta_{*}} \psi_{k}\left(x_{1}, x_{2}\right)\right\|=H\left(c, \Delta_{*}\right), \quad c=\left(x_{2}^{\theta}, x_{1}^{\theta}, x_{1}, x_{2}\right) .
$$

Vladimirov's theorem 4.1 remains true for Hilbert-space-valued holomorphic functions, whose norm satisfies a powerlaw bound in the forward tube. Applying such a version of theorem 4.1, as well as arguments from the proof of theorem 8.1 and from section 8.1.1 (in particular using the bound (8.16)), it's easy to obtain the following result (we omit the proof).

Theorem 8.4. (a) The boundary value $\operatorname{bv}(\psi)=\lim _{\epsilon_{i} \rightarrow 0} \psi\left(x_{1}, x_{2}\right)$ exists as $\mathcal{H}^{\mathrm{CFT}}$-valued tempered distributions, and similarly for each $\operatorname{bv}\left(\psi_{k}\right): \operatorname{bv}(\psi), \operatorname{bv}\left(\psi_{k}\right) \in \mathcal{S}^{\prime}\left(\mathbb{R}^{2 d}\right) \otimes \mathcal{H}^{\mathrm{CFT}}$. Explicitly, the limit

$$
(\operatorname{bv}(\psi), f)=\lim _{\epsilon \rightarrow 0} \int \psi(\epsilon+i t, \mathbf{x}) f(t, \mathbf{x}) d^{2} t d^{2(d-1)} \mathbf{x}
$$

exists as a vector in $\mathcal{H}^{\mathrm{CFT}}$ for any Schwartz function $f \in \mathcal{S}\left(\mathbb{R}^{2 d}\right)$, and is a continuous linear operator from $\mathcal{S}\left(\mathbb{R}^{2 d}\right)$ to $\mathcal{H}^{\mathrm{CFT}}$, and analogously for $\operatorname{bv}\left(\psi_{k}\right)$;

(b) (Distributional OPE convergence in $\mathcal{H}^{\mathrm{CFT}}$ ) For each Schwartz test function $f \in$ $\mathcal{S}\left(\mathbb{R}^{2 d}\right)$, the series $\sum_{k}\left(\mathrm{bv}\left(\psi_{k}\right), f\right)$ converges in $\mathcal{H}^{\mathrm{CFT}}$ norm to $(\mathrm{bv}(\psi), f)$;

(c) (Convergence rate) For compactly supported test functions, the series in (b) summed over $\Delta_{k} \leqslant \Delta_{*}$ converges with rate $O\left(\sqrt{\frac{\log \Delta_{*}}{\Delta_{*}}}\right)$. 
Remark 8.1. The following more finegrained version of theorem 8.4(b) also holds. Denote

$$
E_{k, n}\left(x_{1}, x_{2}\right)=f_{\mathcal{O O} k} \tilde{C}_{k, n}\left(x_{1}, x_{2}\right) e_{k, n} .
$$

As explained above, $\tilde{C}_{k, n}\left(x_{1}, x_{2}\right)$ is a finite sum of terms like $\left(\mathcal{D}^{\theta}\right)^{\alpha}\left\langle\left(\mathcal{O}_{k}\right)^{(\lambda)}\left(x_{N}\right) \mathcal{O}\left(x_{1}\right) \mathcal{O}\left(x_{2}\right)\right\rangle$ (all having the same $\left.|\alpha|\right)$, so $\operatorname{bv}\left(E_{k, n}\right)$ exists by Vladimirov's theorem. Then for each Schwartz test function $f \in \mathcal{S}\left(\mathbb{R}^{2 d}\right)$, the series $\sum_{k, n}\left(\mathrm{bv}\left(E_{k, n}\right), f\right)$ converges in $\mathcal{H}^{\mathrm{CFT}}$ norm to $(\mathrm{bv}(\psi), f)$.

It would be interesting to prove a version theorem 8.4(c), truncating the series $\sum_{k, n}\left(\operatorname{bv}\left(E_{k, n}\right), f\right)$ to $k, n$ such as the corresponding $\Delta_{k}+|\alpha| \leqslant \Delta_{*}$. To do so one would have to find an analogue of the bounds (8.31), (8.16) valid for such a truncation. This is not straightforward because the partial sums of the series (8.28) truncated to $\Delta_{k}+|\alpha| \leqslant \Delta_{*}$ do not correspond to a simple truncation of the $\rho, \bar{\rho}$ series of the full 4-point function (basically because the transformation which maps $x_{1}, x_{2}, x_{1}^{\theta}, x_{2}^{\theta}$ to their $\rho, \bar{\rho}$ conformal frame does not necessarily map $x_{S}, x_{N}$ to $\left.0, \infty\right)$.

\subsection{Comparison to Mack's work on OPE convergence}

To assume OPE convergence as an axiom in Euclidean CFT, and to derive Minkowski physics from it, as we did in this paper, seems natural from the modern perspective. On the contrary, in the early days of CFTs it was considered natural to assume standard Minkowski physics (such as Wightman axioms). The OPE convergence was not assumed at the time, but was something to be derived.

This was the underlying philosophy of the works by Lüscher and Mack [71] and of Mack [41, 81]. Written 45 years ago, these papers remain widely cited, but not everyone is familiar with what precisely has been derived there. Here we will present a short review for the benefit of the modern audience.

These works make two main assumptions. First, that we have a unitary quantum field theory in the Minkowski signature which satisfies Wightman axioms (in particular has a Hilbert space $\mathcal{H}$ on which the Poincaré group acts unitarily and quantum fields are operator-valued distributions). Correlators then have the usual analyticity properties of Wightman functions, in particular they are real-analytic in the Euclidean. The second main assumption is that these Euclidean correlators are invariant under the action of the Euclidean conformal group.

Using these two assumptions, Lüscher and Mack [71] proved that the Hilbert space $\mathcal{H}$ carries a unitary representation not just of the Poincaré but of the group $G^{*}=$ universal cover of the Lorentzian conformal group $\mathrm{SO}(d, 2) .{ }^{68}$ Mack [81] then classified all unitary positive energy representations of $G^{*}$. It should be mentioned that refs. [41, 71, 81]

\footnotetext{
${ }^{68}$ One also often quotes Lüscher and Mack [71] for proving that CFT correlation functions may be continued to the Minkowski cylinder $S^{d-1} \times \mathbb{R}$. This is a misquotation as they did not prove this, but posed it as a conjecture. What they did prove was that CFT correlation functions can be analytically continued to a domain of which $S^{d-1} \times \mathbb{R}$ is a real boundary. One still needs to establish a powerlaw bound to take the boundary limit and obtain a distribution. We plan to derive this fact in our future work [72], for 4-point functions, from the Euclidean CFT axioms, using the $\rho, \bar{\rho}$ expansion.
} 
only consider $d=4$ spacetime dimensions. Many explicit group theoretic calculations are done only for this value of $d$. The results should however generalize to arbitrary $d$ with appropriate modifications.

Continuing this program, Mack [41] studied distributional OPE convergence in Minkowski CFT. Since we also have results of this kind (section 8.2), it will be particularly interesting to compare with Mack's discussion. So let us review his argument. Compared to [71, 81], ref. [41] includes one extra assumption: that the OPE $\varphi_{i}\left(x_{1}\right) \varphi_{j}\left(x_{2}\right)$ of two fields acting on the Minkowski vacuum is valid in an asymptotic sense. Namely that for some dense set of states $\psi$ we have ${ }^{69}$

$$
\left\langle\psi \mid \varphi_{i}\left(x_{1}\right) \varphi_{j}\left(x_{2}\right)\right\rangle \sim \sum_{k} C_{i j k}\left(x_{1}, x_{2}\right)\left\langle\psi \mid \varphi_{k}(0)\right\rangle,
$$

as an asymptotic expansion for rescaling $x_{1}, x_{2} \rightarrow \lambda x_{1}, \lambda x_{2}$, where $C_{i j k}\left(x_{1}, x_{2}\right)$ are some $\psi$ independent homogeneous functions: $C_{i j k}\left(\lambda x_{1}, \lambda x_{2}\right)=\lambda^{\Delta_{k}-\Delta_{i}-\Delta_{j}} C_{i j k}\left(x_{1}, x_{2}\right) .{ }^{70}$ Asymptotic means that if we truncate the expansion at $\Delta_{k}=\Delta_{*}$ and take $\lambda \rightarrow 0$ limit for any fixed $x_{1}, x_{2}$ then the error is $o\left(\lambda^{\Delta_{*}-\Delta_{i}-\Delta_{j}}\right)$. Note that there are both primaries and descendants among $\varphi_{k}$ 's. The main result of [41] is to convert this asymptotic expansion to an expansion convergent in the Hilbert space sense.

The first step is to use a general result that any Hilbert space carrying a unitary representation of a semisimple Lie group can be decomposed as a direct integral of unitary irreducible representations [82]. Since, by the above-mentioned result of [71], $\mathcal{H}$ carries a unitary representation of $G^{*}$, we thus have

$$
\mathcal{H}=\int^{\oplus} d \mu_{\chi} d \tilde{\mu}_{\nu} \mathcal{H}^{\chi \nu}
$$

where $\chi$ labels different unitary irreps of $G^{*}, \chi=\left(\Delta_{\chi}, \rho_{\chi}\right)$ with $\Delta_{\chi}$ the scaling dimension and $\rho_{\chi}$ a Lorentz group irrep, and $\nu$ labels different copies of the same irrep. Only positive energy irreps may occur, since all states of Wightman theory have positive energy. By definition, eq. (8.35) identifies every vector $\psi \in \mathcal{H}$ with a Hilbert-space-valued function $(\chi, \nu) \mapsto \psi_{\chi \nu} \in \mathcal{H}^{\chi}$, some standard realization of the irrep $\chi$. It is assumed that $\langle\psi \mid \psi\rangle<\infty$, inner products being given by the following integral:

$$
\left\langle\psi \mid \psi^{\prime}\right\rangle=\int d \mu_{\chi} d \tilde{\mu}_{\nu}\left\langle\psi_{\chi \nu} \mid \psi_{\chi \nu}^{\prime}\right\rangle_{\mathcal{H} \chi},
$$

Also $G^{*}$ acts on $\psi$ by acting on each $\psi_{\chi \nu}$. Furthermore, ref. [81] realized $\mathcal{H}^{\chi}$ as a space of distributions $\psi(x)$ on Minkowski space ${ }^{71}$ taking values in the representation space of $\rho_{\chi}$, with Fourier transform supported in the forward light cone, and for which the following inner product (defined initially on a dense subset of smooth $\psi, \psi^{\prime}$ ) is finite:

$$
\left\langle\psi \mid \psi^{\prime}\right\rangle_{\mathcal{H} \chi}=\int d x d y \overline{\psi(x)} I^{\chi}(x-y) \psi^{\prime}(y)
$$

\footnotetext{
${ }^{69}$ Mack assumes $x_{2}=-x_{1}$ but here for simplicity we will assume that this is valid for any $x_{1}, x_{2}$.

${ }^{70} \mathrm{In}$ fact $C_{i j k}$ is a distribution so homogeneity should be understood in the sense of pairing with a rescaled test function.

${ }^{71}$ More properly $\psi(x)$ is a distribution on the Lorentzian cylinder on which the group $G^{*}$ acts naturally, but due to a periodicity condition it may be reconstructed from its values on the Poincaré patch.
} 
where $I^{\chi}$ is an "intertwining kernel". Physically, $I^{\chi}$ is the Minkowski CFT 2-point function of the primary in the "shadow irrep" of $\chi$.

The above integration measure $d \mu_{\chi} d \tilde{\mu}_{\nu}$ depends on the theory; from the abstract arguments alone it may be continuous or discrete. Ref. [41] then proceeds to show that (a) this measure is actually discrete (a sum of delta-functions), so that the direct integral is a direct sum; (b) that the state $\left|\mathcal{O}_{i}\left(x_{1}\right) \mathcal{O}_{j}\left(x_{2}\right)\right\rangle$ produced by two Minkowski primary operators acting on the Minkowski vacuum can be written as

$$
\left|\mathcal{O}_{i}\left(x_{1}\right) \mathcal{O}_{j}\left(x_{2}\right)\right\rangle=\sum_{k, a} f_{i j k}^{a} \int d x B_{k, i j}^{a}\left(x, x_{1}, x_{2}\right)\left|\mathcal{O}_{k}(x)\right\rangle
$$

where $B_{i j k}^{a}\left(x, x_{1}, x_{2}\right), a=1 \ldots N_{i j k}$, are some kinematically determined distributions, the convergence is in the Hilbert space sense after integrating with an arbitrary test functions $f\left(x_{1}, x_{2}\right)$, and the local primary operators $\mathcal{O}_{k}$ occurring in this sum have quantum numbers in the discrete set where the integration measure $d \mu_{\chi} d \tilde{\mu}_{\nu}$ is supported.

To show how this comes about, let us focus on the case of scalar identical $\mathcal{O}_{i}=\mathcal{O}_{j}=\mathcal{O}$ for simplicity. In this case expansion (8.38) will end up being precisely our expansion (8.30) (although derived under very different assumptions), with $B\left(x, x_{1}, x_{2}\right)$ related to the OPE kernel in (8.26).

Applying (8.36) with $\left|\psi^{\prime}\right\rangle=\left|\mathcal{O}\left(x_{1}\right) \mathcal{O}\left(x_{2}\right)\right\rangle$ gives (eq. (2.6) in [41]):

$$
\left\langle\psi \mid \mathcal{O}\left(x_{1}\right) \mathcal{O}\left(x_{2}\right)\right\rangle=\int d \mu_{\chi} d \tilde{\mu}_{\nu} c_{\chi \nu} \int d x \overline{\psi_{\chi \nu}(x)} B_{\chi}\left(x, x_{1}, x_{2}\right),
$$

where we denoted $\int d y I^{\chi}(x-y) \psi_{\chi \nu}^{\prime}(y)=c_{\chi \nu} B_{\chi}\left(x, x_{1}, x_{2}\right)$ where $c_{\chi \nu}$ is a proportionality factor, and $B_{\chi}\left(x, x_{1}, x_{2}\right)$ is a kinematically determined distribution (it is basically the amputated Minkowski 3-point function $\left.\left\langle\mathcal{O}_{\chi}(x) \mathcal{O}\left(x_{1}\right) \mathcal{O}\left(x_{2}\right)\right\rangle\right)$. Mack then undertakes a meticulous study of $B_{\chi}\left(x, x_{1}, x_{2}\right)$ and of its Fourier transform with respect to the first argument $\widehat{B}_{\chi}\left(p, x_{1}, x_{2}\right)$. This actually takes most of his paper, and involves many explicit nontrivial calculations (e.g. it involves the first ever explicit characterization of the most general 3-point function of CFT primaries in arbitrary irreps). One of the main results is that $\widehat{B}_{\chi}\left(p, x_{1}, x_{2}\right)$ are entire functions of $p$ :

$$
\widehat{B}_{\chi}\left(p, x_{1}, x_{2}\right)=\sum_{|\alpha| \geqslant 0} b_{\chi}^{\alpha}\left(x_{1}, x_{2}\right)(-i p)_{\alpha}
$$

where $b_{\chi}^{\alpha}\left(x_{1}, x_{2}\right)=\left(x_{12}^{2}\right)^{-\Delta_{\mathcal{O}}+\Delta_{\chi} / 2}$ times a polynomial in $x_{1}, x_{2}$ of degree $|\alpha|$, in particular $b_{\chi}^{\alpha}\left(\lambda x_{1}, \lambda x_{2}\right)=\lambda^{\Delta_{\chi}+|\alpha|-2 \Delta_{\mathcal{O}}} b_{\chi}^{\alpha}\left(x_{1}, x_{2}\right)$.

Let us now specialize to states $\psi$ for which the function $\psi_{\chi \nu}(x)$ has Fourier transform of compact support (one can show that such states are dense in $\mathcal{H}$ ). Then the previous equations imply the following convergent expansion for the integrand in (8.39):

$$
\int d x \overline{\psi_{\chi \nu}(x)} B_{\chi}\left(x, x_{1}, x_{2}\right)=\sum_{\alpha} b_{\chi}^{\alpha}\left(x_{1}, x_{2}\right) \overline{\partial^{\alpha} \psi_{\chi}(0)}
$$

Mack then claims (before eq. $\left(2.11^{\prime}\right)$ ) that if, for each $\chi$, this convergent expansion is truncated at $\Delta_{\chi}+|\alpha|=\Delta_{*}$, and inserted back into (8.39), this results in an asymptotic 
expansion for the l.h.s. of (8.39). I.e. for any $\Delta_{*}$ (Mack does not write this equation explicitly):

$$
\left\langle\psi \mid \mathcal{O}\left(x_{1}\right) \mathcal{O}\left(x_{2}\right)\right\rangle=\left\{\int d \mu_{\chi} d \tilde{\mu}_{\nu} c_{\chi \nu} \sum_{\Delta_{\chi}+|\alpha| \leqslant \Delta_{*}} b_{\chi}^{\alpha}\left(x_{1}, x_{2}\right) \overline{\partial^{\alpha} \psi_{\chi}(0)}\right\}+E\left(x_{1}, x_{2} ; \Delta_{*}\right),
$$

where the error term $E\left(\lambda x_{1}, \lambda x_{2} ; \Delta_{*}\right)=O\left(\lambda^{\Delta_{*}-2 \Delta_{\mathcal{O}}}\right)$ as $\lambda \rightarrow 0$ for any fixed $x_{1}$. Unfortunately, Mack does not give any justification of this claim, which to us does not appear self-evident. The difficulty is that although for every $\chi, \nu$ the truncated series has error $O\left(\lambda^{\Delta_{*}-2 \Delta_{\mathcal{O}}}\right)$, the constant will certainly depend on $\chi, \nu$. How do we know that the error estimate survives after the integration in $\chi, \nu$ ? It might be possible to close this omission in Mack's reasoning using normalizability of $|\psi\rangle$, but this needs extra arguments compared to what is given in his paper, and we have not investigated this in detail. ${ }^{72}$ Researchers relying on Mack's result should keep this caveat in mind.

Assuming that (8.42) is true, the argument is completed as follows. We now have two asymptotic expansions for the 1.h.s. of $\left\langle\psi \mid \mathcal{O}\left(x_{1}\right) \mathcal{O}\left(x_{2}\right)\right\rangle$, one coming from (8.42), and another from (8.34). The second one is discrete (by assumption), so the first one also must be discrete. This establishes that the measure $d \mu_{\chi} d \tilde{\mu}_{\nu}$ is discrete, a sum of delta functions, hence we can write (8.39) with the r.h.s. as a sum, not an integral:

$$
\left\langle\psi \mid \mathcal{O}\left(x_{1}\right) \mathcal{O}\left(x_{2}\right)\right\rangle=\sum_{n} c_{\chi_{n}} \int d x \overline{\psi_{\chi_{n}}(x)} B_{\chi_{n}}\left(x, x_{1}, x_{2}\right) .
$$

A more detailed comparison of this equation with (8.34) leads us to conclude that (a)

$$
c_{\chi_{n}} \overline{\psi_{\chi_{n}}(x)}=f_{n}\left\langle\psi \mid \mathcal{O}_{\chi_{n}}(x)\right\rangle
$$

where $\mathcal{O}_{\chi_{n}}$ are primary operators related by rescaling to a subset of the local operators $\varphi_{k}$, we choose them unit-normalized (hence a coefficient $f_{n}$ ); (b) that all the other operators $\varphi_{k}$ are the descendants $\mathcal{O}_{\chi_{n}}$ 's; and (c) that all coefficients $C_{k}\left(x_{1}, x_{2}\right)$ are basically the expansion coefficients $b_{\chi_{n}}^{\alpha}\left(x_{1}, x_{2}\right)$ in (8.40). From (8.43) and (8.44), we have

$$
\left\langle\psi \mid \mathcal{O}\left(x_{1}\right) \mathcal{O}\left(x_{2}\right)\right\rangle=\sum_{n} f_{n} \int d x\left\langle\psi \mid \mathcal{O}_{\chi_{n}}(x)\right\rangle B_{\chi_{n}}\left(x, x_{1}, x_{2}\right)
$$

for a dense set of states $\psi$. Because of the orthogonality of different $\left|\mathcal{O}_{\chi_{n}}(x)\right\rangle$ 's, this implies that

$$
\left|\mathcal{O}\left(x_{1}\right) \mathcal{O}\left(x_{2}\right)\right\rangle=\sum_{n} f_{n} \int d x B_{\chi_{n}}\left(x, x_{1}, x_{2}\right)\left|\mathcal{O}_{\chi_{n}}(x)\right\rangle
$$

the sum convergent in the Hilbert space sense after integrating out with any test function $f\left(x_{1}, x_{2}\right)$. This is eq. (8.38) in the considered case $\mathcal{O}_{i}=\mathcal{O}_{j}=\mathcal{O}$.

\footnotetext{
${ }^{72}$ We also tried, but unfortunately we did not manage, to get feedback from Prof. Gerhard Mack concerning this matter.
} 


\subsubsection{Relating Mack's kernel $B$ to the Euclidean OPE kernel $C$}

Now we would like to relate Mack's OPE kernel $B$ to our OPE kernel $C_{a,(\lambda)}^{(\mu)(\nu)}\left(x_{1}, x_{2}, x_{0}, \partial_{0}\right)$ defined by eqs. (2.35) and (2.36). We only consider the OPE kernel for the scalar external operators for simplicity, i.e. $C_{\chi}\left(x_{1}, x_{2}, x_{0}, \partial_{0}\right), \chi=(\Delta, \ell=0)$; similar remarks apply in the general case. We first give the conclusion:

$$
C_{\chi}\left(x_{1}, x_{2}, x_{0}, \partial_{0}\right)=\sum_{\mu} b_{\chi}^{\mu}\left(x_{1}-x_{0}, x_{2}-x_{0}\right) \partial_{0}^{\mu},
$$

where the coefficient functions $b_{\chi}^{\mu}$ are the same as in (8.40). One could "derive" this by using (8.38) and formally manipulating the integral in the momentum space: ${ }^{73}$

$$
\begin{aligned}
\left|\mathcal{O}\left(x_{1}\right) \mathcal{O}\left(x_{2}\right)\right\rangle_{\chi} & =\int d x B_{\chi}\left(x, x_{1}, x_{2}\right)\left|\mathcal{O}_{\chi}(x)\right\rangle \\
& =\int d p \widehat{B}_{\chi}\left(p, x_{1}, x_{2}\right)\left|\widehat{\mathcal{O}}_{\chi}(p)\right\rangle=\sum_{\mu} b_{\chi}^{\mu}\left(x_{1}, x_{2}\right)\left|\partial^{\mu} \mathcal{O}_{\chi}(0)\right\rangle,
\end{aligned}
$$

which shows (8.47) in the case when $x_{0}=0$. The general $x_{0}$ case follows by translation invariance. This derivation is not rigorous for various reasons: (a) we did not clarify the meaning of $\widehat{\mathcal{O}}_{\chi}(p)$; (b) why can we swap the order of summation and integration? (c) the above derivation is done in Minkowski region, how do we match the coefficients $b_{\chi}^{\mu}\left(x_{1}, x_{2}\right)$ with the Euclidean coefficients in (2.36)?

Below we will give a rigorous justification of (8.47), using only the two- and 3-point functions which are kinematically determined by conformal invariance. Recall that on the Euclidean side, the formal power series of $C_{\chi}$ (the scalar version of (2.36))

$$
C_{\chi}\left(x_{1}, x_{2}, x_{0}, \partial_{0}\right)=\frac{1}{\left(x_{12}^{2}\right)^{\Delta_{\mathcal{O}}-\Delta_{\chi} / 2}} \sum_{\mu} c_{\chi}^{\mu}\left(x_{10}, x_{20}\right) \partial_{0}^{\mu}
$$

is determined by the Euclidean two- and 3-point functions:

$$
\left\langle\mathcal{O}_{\chi}^{\dagger}(y) \mathcal{O}\left(x_{1}\right) \mathcal{O}\left(x_{2}\right)\right\rangle_{E}=\frac{1}{\left(x_{12}^{2}\right)^{\Delta_{\mathcal{O}-\Delta_{\chi} / 2}}} \sum_{\alpha} c_{\chi, \alpha}\left(x_{10}, x_{20}\right)\left\langle\mathcal{O}_{\chi}^{\dagger}(y) \partial^{\alpha} \mathcal{O}_{\chi}\left(x_{0}\right)\right\rangle_{E}
$$

Here we already used translation invariance, which implies $C_{a,(\lambda), \alpha}^{(\mu)(\nu)}\left(x_{1}, x_{2}, x_{0}\right)=$ $c_{a,(\lambda), \alpha}^{(\mu)(\nu)}\left(x_{10}, x_{20}\right)$ on the r.h.s. of (2.36). One can match the coefficients $c_{\chi}^{\mu}\left(x_{10}, x_{20}\right)$ with the Taylor expansion of $\left\langle\mathcal{O}_{\chi}^{\dagger}(y) \mathcal{O}\left(x_{1}\right) \mathcal{O}\left(x_{2}\right)\right\rangle_{E}$ around $x_{1}=x_{2}=x_{0}$. In Euclidean one can always find a proper region for the matching: let $y$ be sufficiently far from the $\left(x_{0}, x_{1}, x_{2}\right)$ cluster, so that the Taylor expansions of $\left[\left(y-x_{1}\right)^{2}\right]^{\#}$ around $x_{1}=x_{0}$ and $\left[\left(y-x_{2}\right)^{2}\right]^{\#}$ around $x_{2}=x_{0}$ are convergent.

On the Minkowski side, the OPE kernel $B_{\chi}$ is kinematically determined by the equality

$$
\widehat{G}_{\mathcal{O}_{\chi} \mathcal{O O}}\left(p, x_{1}, x_{2}\right)=\widehat{B}_{\chi}\left(p, x_{1}, x_{2}\right) \widehat{G}_{\chi}(p),
$$

\footnotetext{
${ }^{73}$ Since here we are only interested in the OPE kernels $B_{\chi}$ and $C_{\chi}$, we set $f_{\chi}=1$ (the overall coefficient) for convenience.
} 
where $\widehat{G}_{\mathcal{O}_{\chi} \mathcal{O O}}\left(p, x_{1}, x_{2}\right)=\int d y\left\langle\mathcal{O}_{\chi}^{\dagger}(y) \mathcal{O}\left(x_{1}\right) \mathcal{O}\left(x_{2}\right)\right\rangle_{M} e^{-i p \cdot y} \quad$ and $\quad \widehat{G}_{\chi}(p)=$ $\int d y\left\langle\mathcal{O}_{\chi}^{\dagger}(y) \mathcal{O}_{\chi}(0)\right\rangle_{M} e^{-i p \cdot y}$ (see [41], eq. (8.2)). ${ }^{74}$ All Fourier transforms here are in the sense of distributions. To get an equation valid in the sense of functions we pick a test function $\varphi$ with compactly supported Fourier transform, and integrate (8.50) against $\widehat{\varphi}$, which gives:

$$
\int\left\langle\mathcal{O}_{\chi}^{\dagger}(x) \mathcal{O}\left(x_{1}\right) \mathcal{O}\left(x_{2}\right)\right\rangle_{M} \varphi(x) d x=\int d p \widehat{\varphi}(p) \widehat{B}_{\chi}\left(p, x_{1}, x_{2}\right) \widehat{G}_{\chi}(p) .
$$

The variable $x$ ranges over the Minkowski space, while we will pick $x_{1}, x_{2}$ complex, in the forward tube region

$$
\operatorname{Im}\left(x_{1}\right), \operatorname{Im}\left(x_{2}\right) \prec 0 .
$$

Then the 3-point function $\left\langle\mathcal{O}_{\chi}^{\dagger}(x) \mathcal{O}\left(x_{1}\right) \mathcal{O}\left(x_{2}\right)\right\rangle_{M}$ is nonsingular as a function of $x$ and the 1.h.s. of (8.51) is a finite number. To transform the r.h.s. of (8.51) we will use the fact that $\widehat{B}_{\chi}$ has the following form (a more detailed version than $(8.40)$ ):

$$
\widehat{B}_{\chi}\left(p, x_{1}, x_{2}\right)=\frac{e^{-i p \cdot x_{1}}}{\left(x_{12}^{2}\right)^{\Delta_{\mathcal{O}}-\Delta_{\chi} / 2}} E_{\chi}\left(x_{12} \cdot p, x_{12}^{2} p^{2}\right)
$$

where $E_{\chi}\left(z_{1}, z_{2}\right)$ is some entire function on $\mathbb{C}^{2}$. Hence as long as $x_{12}^{2} \neq 0$ (not necessarily real), $\widehat{B}_{\chi}\left(p, x_{1}, x_{2}\right)$ has the following convergent expansion:

$$
\widehat{B}_{\chi}\left(p, x_{1}, x_{2}\right)=\frac{e^{-i p \cdot x_{1}}}{\left(x_{12}^{2}\right)^{\Delta_{\mathcal{O}-\Delta_{\chi} / 2}}} \sum_{\alpha} a_{\chi}^{\alpha}\left(x_{21}\right)(-i p)_{\alpha},
$$

where $a_{\chi}^{\alpha}(x)$ is some $\mathrm{SO}(1, d-1)$-covariant, homogeneous, symmetric polynomial of degree $|\alpha|$. Plugging this into (8.51), using that the expansion (8.54) converges uniformly on the support of $\widehat{\varphi}$ (assumed compact), and the fact that $\widehat{G}_{\chi}$ is a tempered measure, ${ }^{75}$ we obtain:

$$
\begin{aligned}
\int\left\langle\mathcal{O}_{\chi}^{\dagger}(x) \mathcal{O}\left(x_{1}\right)\right. & \left.\mathcal{O}\left(x_{2}\right)\right\rangle_{M} \varphi(x) d x \\
= & \frac{1}{\left(x_{12}^{2}\right)^{\Delta_{\mathcal{O}}-\Delta_{\chi} / 2}} \sum_{\alpha} a_{\chi}^{\alpha}\left(x_{21}\right) \int\left\langle\mathcal{O}_{\chi}^{\dagger}(x) \partial_{\alpha} \mathcal{O}_{\chi}\left(x_{1}\right)\right\rangle_{M} \varphi(x) d x .
\end{aligned}
$$

At this stage we have established that for any $x_{1}, x_{2}$ as in (8.52), and for any test $\varphi$ with compact $\widehat{\varphi}$, the series in the r.h.s. converges to the l.h.s.

Now the key point is that the r.h.s. of (8.55) is a convergent power series in $x_{21}$, while the l.h.s. can be expanded in such a convergent power series. Indeed we know the explicit form of $\left\langle\mathcal{O}_{\chi}^{\dagger}(x) \mathcal{O}\left(x_{1}\right) \mathcal{O}\left(x_{2}\right)\right\rangle_{M}$ :

$$
\left\langle\mathcal{O}_{\chi}^{\dagger}(x) \mathcal{O}\left(x_{1}\right) \mathcal{O}\left(x_{2}\right)\right\rangle_{M}=\frac{1}{\left(x_{12}^{2}\right)^{\Delta_{\mathcal{O}}-\Delta_{\chi} / 2}} \frac{1}{\left[\left(x-x_{1}\right)^{2}\left(x-x_{2}\right)^{2}\right]^{\Delta_{\chi} / 2}} .
$$

\footnotetext{
${ }^{74}$ In the unitary CFTs, $\widehat{G}_{\bar{n} \mathcal{O O}}\left(p, x_{1}, x_{2}\right)$ and $\widehat{G}_{\chi_{n}}(p)$ vanish unless $p \in \overline{V_{+}}$, so the behavior of $\widehat{B}_{\chi_{n}}\left(p, x_{1}, x_{2}\right)$ outside the forward light cone is not important.

${ }^{75}$ This is a consequence of the Bochner-Schwartz theorem: any positive tempered distribution is the Fourier transform of some positive tempered measure (see [83], section 8.2).
} 
For all $x$ in the Minkowski space, the function $\left[\left(x-x_{1}\right)^{2}\left(x-x_{2}\right)^{2}\right]^{-\Delta_{\chi} / 2}$ is holomorphic in $x_{1}, x_{2}$ as long as $\operatorname{Im}\left(x_{1}\right), \operatorname{Im}\left(x_{2}\right) \prec 0$. It's easy to show that this remains true after integration in $\varphi$. At this point we can match the expansions for the two sides of (8.55), and get

$$
\begin{aligned}
\sum_{|\alpha|=n} \int d x \varphi(x)\left\{\frac { ( x _ { 2 1 } ) _ { \alpha } } { \alpha ! } \partial _ { x _ { 2 } } ^ { \alpha } \left[\left(x-x_{1}\right)^{2}(\right.\right. & \left.\left.x-x_{2}\right)^{2}\right]\left.^{-\Delta_{\chi} / 2}\right|_{x_{2}=x_{1}} \\
& \left.-a_{\chi}^{\alpha}\left(x_{21}\right)\left\langle\mathcal{O}_{\chi}^{\dagger}(x) \partial_{\alpha} \mathcal{O}_{\chi}\left(x_{1}\right)\right\rangle_{M}\right\}=0 .
\end{aligned}
$$

Up to this point it was crucial to keep the function $\varphi$ in the game to keep convergence issues under control, but now we can get rid of it. Indeed $\sum_{|\alpha|=n}$ is a finite sum, also $\left\langle\mathcal{O}_{\chi}^{\dagger}(x) \partial_{\alpha} \mathcal{O}_{\chi}\left(x_{1}\right)\right\rangle_{M}$ is a holomorphic function in the forward tube $\mathcal{T}_{2}$. For any Minkowski point $x_{0}$, we choose a sequence of test functions $\varphi_{k}$ of compact support $\widehat{\varphi}_{k}$ such that $\varphi_{k}$ tends to $\delta\left(x-x_{0}\right)$. Passing to the limit, (8.57) implies the same equality for the integrand. I.e. for any fixed $n \in \mathbb{N}$, and any Minkowski $x$,

$$
\left.\frac{\left(x_{21}\right)_{\alpha}}{\alpha !} \partial_{x_{2}}^{\alpha}\left[\left(x-x_{1}\right)^{2}\left(x-x_{2}\right)^{2}\right]^{-\Delta_{\chi} / 2}\right|_{x_{2}=x_{1}}-a_{\chi}^{\alpha}\left(x_{21}\right)\left\langle\mathcal{O}_{\chi}^{\dagger}(x) \partial_{\alpha} \mathcal{O}_{\chi}\left(x_{1}\right)\right\rangle_{M}=0 .
$$

Now as promised we are reduced to an equation which only involves 2-point and 3-point functions which are holomorphic. E.g. we can take $x=0$ and $x_{1}, x_{2}$ in Euclidean. Then this equation is the same one as the equation which determines the Euclidean OPE kernel for $x_{0}=x_{1}$, i.e. $C_{\chi}\left(x_{1}, x_{2}, x_{1}, \partial\right)$. For convenience in this discussion we use Minkowski coordinates for Euclidean correlators (i.e. we write the Euclidean correlators as $\left\langle\langle\mathcal{O}(-i \tau, \mathbf{x}) \ldots\rangle_{M}\right)$. Under this convention we have

$$
C_{\chi}\left(x_{1}, x_{2}, x_{1}, \partial\right)=\frac{1}{\left(x_{12}^{2}\right)^{\Delta_{\mathcal{O}}-\Delta_{\chi} / 2}} \sum_{(\mu)} a_{\chi}^{\mu}\left(x_{21}\right) \partial_{\mu}=\sum_{(\mu)} b_{\chi}^{\alpha}\left(0, x_{21}\right) \partial_{\alpha}
$$

This establishes (8.47) for $x_{0}=x_{1}$. The general case reduces to this one by noticing that $c_{\chi}^{\alpha}$ satisfies the relation:

$$
c_{\chi}^{\alpha}\left(x_{10}, x_{20}\right)=\sum_{\beta \leqslant \alpha} \frac{1}{\beta !} c_{\chi}^{\alpha-\beta}\left(0, x_{21}\right) x_{10}^{\beta},
$$

where $\beta \leqslant \alpha$ means $\beta_{i} \leqslant \alpha_{i}$ for all $i$; and $b_{\chi}$ satisfies the identical relation with $c_{\chi} \rightarrow b_{\chi}$. For $c_{\chi}^{\alpha}$ this follows by translation invariance and analyticity of CFT two- and 3-point functions, and for $b_{\chi}^{\alpha}$ from $\widehat{B}_{\chi}\left(p, x_{10}, x_{20}\right)=e^{-i p \cdot x_{10}} \widehat{B}_{\chi}\left(p, 0, x_{21}\right)$.

\section{Review of Osterwalder-Schrader theorem}

In this section we review the results of $[2,3]$ and, in particular, discuss the linear growth condition and why it was necessary for establishing Wightman axioms in [3].

In [2] Osterwalder and Schrader formulated an equivalence theorem which stated that a set of axioms for Euclidean correlation functions (a version of the Osterwalder-Schrader axioms described in section 2.2) is equivalent to Wightman axioms for Euclidean correlation 
functions. Unfortunately, later a technical error was discovered in their proof, and in [3] Osterwalder and Schrader gave two new results.

The first result of [3] is a revised equivalence theorem, which shows that a stronger version of Euclidean axioms is in fact equivalent to Wightman axioms. The proof of this theorem is rather simple. However, as we will review, this is at the expense of the new version of Euclidean axioms being rather hard to verify.

The second result of [3] shows that the original OS axioms, plus a "linear growth condition," imply Wightman axioms and a growth condition on Wightman distributions. A partial result in the reverse direction is also valid. It assumes a stronger growth condition on the Wightman distributions than follows from the direct result, and it yields a growth condition on Euclidean correlators which is weaker than the linear growth condition. Therefore, these latter results do not establish an equivalence of any two systems of axioms, but they do allow to establish Wightman axioms from OS axioms in some situations.

In what follows we will review the general structure of the arguments of $[2,3]$. For our purposes it will suffice to ignore the space coordinates and focus only on the time arguments of the fields. We will not completely reproduce all arguments of $[2,3]$, and in some of the omitted steps the space arguments and Lorentz symmetry are important. We will also work with correlation functions involving a single hermitian scalar field $\phi$, similarly to $[2,3]$. In CFT applications we are interested in correlation functions of all local operators. It should be relatively straightforward to adapt the discussion of $[2,3]$ to this more general setup.

Our main goal is to construct an analytic continuation of the Euclidean correlation functions

$$
G_{n}^{E}\left(t_{1}, \ldots, t_{n}\right) \equiv\left\langle\phi\left(t_{1}\right) \ldots \phi\left(t_{n}\right)\right\rangle
$$

from real to complex $t_{k}$, and to establish that the Wightman functions recovered in the limit of pure imaginary $t_{k}$ (real Lorentzian times) are tempered distributions. This is the most non-trivial part of the argument. Other Wightman axioms such as positivity, spectrum condition, etc., follow relatively easily and have been reviewed in section 6 .

\subsection{The argument of [2]}

Physically, the analyticity of position-space correlation functions is due to positivity of energy. More concretely, the Euclidean evolution operator $e^{-H t}$ is well-defined and holomorphic in $t$ for Ret $>0$ due to the spectrum of $H$ being non-negative. The first step to establishing analyticity is thus to construct the operator $H$, and for this we first need to construct a Hilbert space on which it acts.

The Hilbert space $\mathcal{H}^{\mathrm{OS}}$ is constructed, as we discussed in section 2.2, by considering the vector space $\mathcal{H}_{0}^{\mathrm{OS}}$ of formal linear combinations of $\operatorname{states}^{76}$

$$
\left|\phi\left(t_{1}\right) \phi\left(t_{2}\right) \ldots \phi\left(t_{n}\right)\right\rangle
$$

\footnotetext{
${ }^{76}$ In section 2.2 the states are introduced as integrals of these quantities. This is also what is done in [2,3], since they assume only that the Euclidean correlators are distributions. Here, for simplicity of discussion, we use the knowledge that correlators are functions and use states evaluated at points. The arguments easily generalize to distributions and smeared states, but become more technical.
} 
with $0>t_{1}>t_{2}>\cdots>t_{n}$. A Hermitian inner product is introduced on $\mathcal{H}_{0}^{\mathrm{OS}}$ by

$$
\left\langle\phi\left(s_{1}\right) \phi\left(s_{2}\right) \ldots \phi\left(s_{m}\right) \mid \phi\left(t_{1}\right) \phi\left(t_{2}\right) \ldots \phi\left(t_{n}\right)\right\rangle \equiv G_{n}^{E}\left(-s_{m}, \ldots,-s_{1}, t_{1}, \ldots, t_{n}\right) .
$$

By OS reflection positivity-axiom this inner product is positive-semidefinite. The Hilbert space $\mathcal{H}^{\mathrm{OS}}$ is obtained from $\mathcal{H}_{0}^{\mathrm{OS}}$ by modding out null states and completing the resulting quotient space with respect to the above inner product. We can naturally think of $\left|\phi\left(t_{1}\right) \phi\left(t_{2}\right) \ldots \phi\left(t_{n}\right)\right\rangle$ as states in $\mathcal{H}^{\mathrm{OS}}$.

Physically, to construct the Hamiltonian $H$, we first define it by its action on (9.2). Then we note that $H$ has to be positive, otherwise the correlation functions would grow exponentially at large distances. Formally, one first defines for $t>0$ an operator $U_{t}$ on $\mathcal{H}_{0}^{\mathrm{OS}}$ by

$$
U_{t}\left|\phi\left(t_{1}\right) \phi\left(t_{2}\right) \ldots \phi\left(t_{n}\right)\right\rangle \equiv\left|\phi\left(t_{1}-t\right) \phi\left(t_{2}-t\right) \ldots \phi\left(t_{n}-t\right)\right\rangle .
$$

The usual care must be taken to ensure that this defines an operator on $\mathcal{H}^{\mathrm{OS}}$. For this one notes that for any $\Psi \in \mathcal{H}_{0}^{\mathrm{OS}}$ we have $\left|\left\langle\Psi\left|U_{t}\right| \Psi\right\rangle\right| \leqslant P(t)$ for some polynomial $P(t)$ since the Euclidean correlation functions are assumed to be powerlaw-bounded when groups of points are separated to infinity. Then a simple estimate gives

$$
\left|\left\langle\Psi\left|U_{t}\right| \Psi\right\rangle\right| \leqslant\|\Psi\|\left\|U_{t} \Psi\right\|=\|\Psi\|\left|\left\langle\Psi\left|U_{2 t}\right| \Psi\right\rangle\right|^{1 / 2} \leqslant \cdots \leqslant\|\Psi\|^{1+1 / 2+\cdots+1 / 2^{n-1}}\left|\left\langle\Psi\left|U_{2^{n} t}\right| \Psi\right\rangle\right|^{1 / 2^{n}} .
$$

Using $\left|\left\langle\Psi\left|U_{t}\right| \Psi\right\rangle\right| \leqslant P(t)$ we get in the limit $n \rightarrow \infty$

$$
\left|\left\langle\Psi\left|U_{t}\right| \Psi\right\rangle\right| \leqslant\|\Psi\|^{1+1 / 2+\cdots+1 / 2^{n-1}}\left(P\left(2^{n} t\right)\right)^{1 / 2^{n}} \rightarrow\|\Psi\|^{2} .
$$

This shows that $U_{t}$ maps null states to null states and thus is defined on (a dense subset of) $\mathcal{H}^{\mathrm{OS}}$. By the above, it is also a bounded operator, so it extends in a unique way to all of $\mathcal{H}^{\mathrm{OS}}$. Furthermore, noting that it is symmetric, of norm at most 1 , and we have the semigroup law $U_{t} U_{s}=U_{t+s}$, we find that $U_{t}=e^{-H t}$ for a non-negative self-adjoint Hamiltonian $H$ (see, e.g., [84] section 141).

Since the domain in which we need to construct the analytic continuation of $G_{n}^{E}$ is awkward to define in $t_{k}$ variables, we introduce the difference variables $y_{k} \equiv t_{k}-t_{k+1}$. Due to translation invariance, $G_{n}^{E}\left(t_{1} \ldots t_{n}\right)$ can be rewritten as

$$
G_{n}^{E}\left(t_{1} \ldots t_{n}\right)=S_{n-1}\left(y_{1} \ldots y_{n-1}\right)
$$

for some functions $S_{n}$. Similarly, we will use the following notation for states in terms of $y_{k}$ variables,

$$
\left|\Psi_{n}\left(-t_{1} ; y_{1} \ldots y_{n-1}\right)\right\rangle \equiv\left|\phi\left(t_{1}\right) \phi\left(t_{2}\right) \ldots \phi\left(t_{n}\right)\right\rangle
$$

Note that

$$
\left\langle\Psi_{m}\left(t^{\prime} ; y_{1}^{\prime} \ldots y_{m-1}^{\prime}\right) \mid \Psi_{n}\left(t ; y_{1} \ldots y_{n-1}\right)\right\rangle=S_{m+n-1}\left(y_{m-1}^{\prime}, \ldots, y_{1}^{\prime}, t^{\prime}+t, y_{1}, \ldots, y_{n-1}\right) .
$$


In terms of $S_{n-1}\left(y_{1} \ldots y_{n-1}\right)$, our goal is to construct an analytic continuation to $\operatorname{Re} y_{k}>0$ and show that the limit of all $\operatorname{Re} y_{k} \rightarrow 0^{+}$exists in the sense of tempered distributions.

With a positive $H$ now constructed, we can define a holomorphic family of bounded operators $U_{\tau}=e^{-H \tau}$ for $\operatorname{Re} \tau>0$, which will be our main tool for analytically continuing the correlation functions $S_{n}$. In particular, we can now consider the matrix elements

$$
\left\langle\Psi_{m}\left(t^{\prime} ; y_{1}^{\prime} \ldots y_{m-1}^{\prime}\right)\left|U_{\tau}\right| \Psi_{n}\left(t ; y_{1} \ldots y_{n-1}\right)\right\rangle=S_{m+n-1}\left(y_{m-1}^{\prime}, \ldots, y_{1}^{\prime}, t^{\prime}+t+\tau, y_{1}, \ldots, y_{n-1}\right)
$$

which are analytic for $\operatorname{Re} \tau>0$. This establishes the desired analyticity of $S_{n-1}\left(y_{1} \ldots y_{n-1}\right)$ in each variable $y_{k}$ separately. In [2] they additionally establish some growth conditions on these individual holomorphic functions which then imply that for fixed $y_{k}, y_{k}^{\prime}$ and $\operatorname{Re} \tau>0$ the above function can be represented as the Fourier-Laplace transform

$$
S_{m+n-1}\left(y_{m-1}^{\prime}, \ldots, y_{1}^{\prime}, \tau, y_{1}, \ldots, y_{n-1}\right)=\int d \alpha e^{-\alpha \tau} \check{S}(\alpha)
$$

for some tempered distribution $\check{S}(\alpha)$. In other words, $S_{m+n-1}$ can be extended to a holomorphic function in the right-half plane in each variable separately, and each such holomorphic function can be represented as a Fourier-Laplace transform of a tempered distribution. The erroneous lemma 8.8 of [2] states that under these conditions, the full function $S_{m+n-1}$ is a simultaneous Fourier-Laplace transform in all its variables of a tempered distribution,

$$
S_{m+n-1}\left(\tau_{1} \ldots \tau_{m+n-1}\right)=\int d \alpha e^{-\alpha_{1} \tau_{1}-\cdots-\alpha_{m+n-1} \tau_{m+n-1}} \check{S}_{m+n-1}\left(\alpha_{1} \ldots \alpha_{m+n-1}\right) .
$$

From this, the tempered Wightman distributions are obtained immediately by setting $\operatorname{Re} \tau_{k} \rightarrow 0^{+}$in which case the Fourier-Laplace transform above becomes a Fourier transform of a tempered distribution. Fourier transform of a tempered distribution is, of course, itself tempered.

\subsection{The argument of [3]}

\subsubsection{Fixing the equivalence theorem}

Unfortunately, lemma 8.8 of [2] is wrong. As explained in [3], the function $S_{2}\left(y_{1}, y_{2}\right)=$ $e^{-y_{1} y_{2}}$ gives a simple counter-example. For fixed $y_{2}>0$,we find that $S_{2}\left(y_{1}, y_{2}\right)$ is holomorphic for Re $y_{1}>0$ and is there the Fourier-Laplace transform of the tempered distribution $\delta\left(\alpha-y_{2}\right)$. The same statements hold with $y_{1}$ and $y_{2}$ exchanged. However, $S_{2}\left(y_{1}, y_{2}\right)$ is not a Fourier-Laplace transform of a tempered distribution in both variables simultaneously. For if this were the case, the corresponding Wightman function $S_{2}\left(i x_{1}, i x_{2}\right)=e^{x_{1} x_{2}}$ would be a tempered distribution, which it is not since it grows faster than any power in some directions.

The first result of [3] (see also the review in [43]) rescues lemma 8.8 by making a stronger assumption about $S_{n}\left(y_{1} \ldots y_{n}\right)$ which they denote by $\check{E} 0$. Concretely, let $\mathbb{R}_{+}^{n}$ be the set of points $\left(y_{1}, \ldots, y_{n}\right)$ with $y_{k}>0$. Let $\mathcal{S}\left(\mathbb{R}_{+}^{n}\right)$ be the subspace of the space of Schwartz functions, consisting of functions supported on $\mathbb{R}_{+}^{n}$ with the induced topology. 
The functions $S_{n}\left(y_{1} \ldots y_{n}\right)$ can be viewed as distributions in the continuous dual space $\mathcal{S}^{\prime}\left(\mathbb{R}_{+}^{n}\right)$ defined by, for $f \in \mathcal{S}\left(\mathbb{R}_{+}^{n}\right)$

$$
S_{n}(f) \equiv \int d y_{1} \ldots d y_{n} S_{n}\left(y_{1} \ldots y_{n}\right) f\left(y_{1} \ldots y_{n}\right) .
$$

Note that smoothness of $f$ together with its support properties ensures that $f\left(y_{1} \ldots y_{n}\right)$ vanishes with all derivatives whenever $y_{k}=y_{j}$ for $k \neq j$. The assumption that $S_{n}$ has at most powerlaw singularities at coincident points and at infinity means that $S_{n}(f)$ is continuous in $f$ in the topology of $\mathcal{S}\left(\mathbb{R}_{+}^{n}\right)$. The additional assumption $\check{E} 0$ is that it is also continuous in $f$ in a weaker topology. This weaker topology is defined by the usual Schwartz norms on $\overline{\mathbb{R}_{+}^{n}}$

$$
|g|_{p,+}=\sup _{x \in \overline{\mathbb{R}_{+}^{n}},|\alpha| \leqslant p}\left|\left(1+x^{2}\right)^{p / 2} \partial^{(\alpha)} g(x)\right|
$$

but applied not to $f$ and instead to its Fourier-Laplace ${ }^{77}$ transform $\check{f}$

$$
\check{f}\left(q_{1} \ldots q_{n}\right) \equiv \int d y_{1} \ldots d y_{n} e^{-q_{1} y_{1}-\cdots-q_{n} y_{n}} f\left(y_{1} \ldots y_{n}\right) .
$$

One establishes that $\check{f}=0$ iff $f=0$ (injectivity) and that the set of all images $\check{f}$ is dense in an appropriate space of Schwartz functions (denseness). The proof of (9.12) then becomes straightforward: one first defines $\check{S}_{n}$ by $\check{S}_{n}(\check{f})=S_{n}(f)$. This definition makes sense due to the injectivity property. The assumption $\check{E} 0$ ensures that $\check{S}_{n}$ is continuous. The denseness property just mentioned then allows to extend $\check{S}_{n}$ to an appropriate space of Schwartz functions by continuity, establishing temperedness of $\check{S}_{n}$ and allowing one to define tempered Wightman distributions as Fourier transforms of $\check{S}_{n}$. It is similarly not difficult to show that Wightman axioms imply $\check{E} 0$.

\subsubsection{Wightman axioms from linear growth condition}

As we can see, the axiom $\check{E} 0$ is not very different from directly assuming temperedness of Wightman distributions, even though it is formulated for Euclidean correlators. It is also unclear how to verify this axiom in practice. ${ }^{78}$ For this reason, [3] introduced an alternative "linear growth condition" on the correlation functions $S_{n}$ which is easier to verify and has been established in some models (see below), yet is also sufficient to establish temperedness of Wightman functions (though this condition is not known to follow from Wightman axioms). The construction of the analytic continuation of the functions $S_{n}$ as

\footnotetext{
${ }^{77}$ As written, this is a Laplace transform. It is a Fourier transform in the spatial variables which we are omitting.

${ }^{78}$ We would also like to mention related work by Zinoviev [85]. Zinoviev replaces axiom Ě0 by an axiom E5 which imposes that certain limits exist which allow to compute the inverse Laplace transform of $S_{n}$. While E5 may look like a more constructive version of $\check{E} 0$, in practice its verification appears just as hard as assuming outright that $S_{n}$ is a Laplace transform (which is what $\check{E} 0$ essentially does). We are grateful to David Brydges for an enlightening explanation of Zinoviev's construction, and in particular for pointing out that it represents a generalization of Post's Laplace transform inversion formula [86] to the case of distributions.
} 
well the proof of the temperedness of the resulting Wightman distributions is much more complicated than using $\check{E} 0$. Therefore, our review of these arguments will be even more schematic than the above, and we will only try to illustrate the key ideas and explain why and how the linear growth condition is used. We are not aware of any previous attempt to review this part of [3].

First of all, let us state the linear growth condition of [3]. Note that the correlation functions $G_{n}^{E}$ can be viewed as distributions in $\left({ }^{0} \mathcal{S}\right)^{\prime}\left(\mathbb{R}^{d \cdot n}\right)$, where ${ }^{0} \mathcal{S}\left(\mathbb{R}^{d \cdot n}\right)$ is the space of Schwartz functions of $n$ arguments in $\mathbb{R}^{d}$ which vanish with all derivatives at coincident points, by

$$
G_{n}^{E}(f)=\int d^{d} x_{1} \ldots d^{d} x_{n} f\left(x_{1}, \ldots, x_{n}\right) G_{n}^{E}\left(x_{1}, \ldots, x_{n}\right) .
$$

Here we have temporarily restored the spatial coordinates. In fact, $[2,3]$ do not assume that $G_{n}^{E}$ are functions, and only that they are distributions in $\left({ }^{0} \mathcal{S}\right)^{\prime}\left(\mathbb{R}^{d \cdot n}\right)$. It follows, however, from the OS axioms (without the linear growth condition) that $G_{n}^{E}$ are realanalytic functions, as shown in [3, 46].

Note that the assumption that $G_{n}^{E} \in\left({ }^{0} \mathcal{S}\right)^{\prime}\left(\mathbb{R}^{d \cdot n}\right)$ means $G_{n}^{E}$ is sufficiently continuous as a linear functional or, equivalently, is sufficiently bounded. That is,

$$
\left|G_{n}^{E}(f)\right| \leqslant \sigma_{n}|f|_{q_{n}}
$$

for all $f \in{ }^{0} \mathcal{S}\left(\mathbb{R}^{d \cdot n}\right)$ and some $\sigma_{n}>0$ and $q_{n} \in \mathbb{Z}_{\geqslant 0}$, where $|f|_{p}$ denotes the Schwartz norms on ${ }^{0} \mathcal{S}\left(\mathbb{R}^{d \cdot n}\right)$. The linear growth condition requires $q_{n}$ to grow at most linearly, and $\sigma_{n}$ at most as a power of a factorial. In other words, the linear growth condition is the statement that there exists a positive integer $s$ and a sequence $\sigma_{n}$ such that

$$
\left|G_{n}^{E}(f)\right| \leqslant \sigma_{n}|f|_{n \cdot s}
$$

for any $n$ and $f \in{ }^{0} \mathcal{S}\left(\mathbb{R}^{d \cdot n}\right)$, and $\sigma_{n} \leqslant \alpha(n !)^{\beta}$ for some constants $\alpha, \beta$.

The unusual feature of the linear growth condition is that this is a condition on all $n$-point correlation functions $G_{n}^{E}$. It has to hold for all $n$ in order for the result of [3] to imply, say, even just the temperedness of 3-point Wightman distribution. In order to understand why this is required, below we will review the basic strategy behind the proof of [3]. There are two steps in the argument. In the first step, one establishes analyticity of $S_{n}\left(y_{1}, \ldots, y_{n}\right)$ in the region $\operatorname{Re} y_{k}>0$. This does not require the linear growth condition $[3,46]$. In the second step, which does use the linear growth condition, one proves a bound on $S_{n}$ in this region, which allows the application of Vladimirov's theorem and thus the construction of tempered Wightman distributions.

We conclude this section with additional comments about the linear growth condition. First of all, appendix of [3] shows that the linear growth condition follows from requiring that $G_{n}^{E} \in \mathcal{S}^{\prime}\left(\mathbb{R}^{d n}\right)$ and imposing

$$
\left|G_{n}^{E}\left(f_{1} \otimes \ldots \otimes f_{n}\right)\right| \leqslant \sigma_{n} \prod_{i=1}^{n}\left|f_{i}\right|_{r},
$$


for any $n$, where $f_{i} \in \mathcal{S}\left(\mathbb{R}^{d}\right),|\cdot|_{r}$ is some fixed Schwartz space norm, and $\sigma_{n} \leqslant \alpha(n !)^{\beta}$. In other words, while in (9.18) the $n$-point function variables are smeared jointly, here each variable is smeared separately. Note that the total smearing $f_{1} \otimes \ldots \otimes f_{n}$ does not necessarily exclude coincident points, that's why we need to assume $G_{n}^{E} \in \mathcal{S}^{\prime}\left(\mathbb{R}^{d n}\right)$ and not $G_{n}^{E} \in\left({ }^{0} \mathcal{S}\right)^{\prime}\left(\mathbb{R}^{d \cdot n}\right)$ as above.

Although (9.19) is stronger than (9.18), it is easier to verify in particular models. E.g. it holds for any gaussian scalar field $\mathcal{O}$ with a two point function $G_{2}$ having a powerlaw asymptotics in the UV. ${ }^{79}$ It has been also established in some non-gaussian models. ${ }^{80}$

More generally, bound (9.19) is natural for field theories realizable as random distributions. ${ }^{81}$ Imagine that there is a measure $d \mu$ in the space of distributions $\phi \in \mathcal{S}^{\prime}\left(\mathbb{R}^{d}\right)$ such that for every test function $f \in \mathcal{S}\left(\mathbb{R}^{d}\right)$ the following expectation value is finite:

$$
S(f)=\int d \mu e^{\phi(f)} .
$$

Such measures make rigorous sense of the Feynman path integral. Eq. (9.20) is a rigorous version of generating functional, and differentiating with respect to $f$ one defines correlation functions $\left\langle\phi\left(x_{1}\right) \ldots \phi\left(x_{n}\right)\right\rangle$ which are in this framework automatically distributions in $\mathcal{S}^{\prime}\left(\mathbb{R}^{d n}\right)$. Bound (9.19) in this case can be reduced to an estimate on the growth of $S(f)$. The Osterwalder-Schrader and Wightman axioms then follow.

The field $\phi$ in (9.20) is naturally a "fundamental field" of some model, such as $P(\phi)_{2}$ [88] or $\left(\phi^{4}\right)_{3}$ (see [88], section 23.1 for references). Sometimes this framework can be extended to generating functionals $\int d \mu e^{\phi^{\prime}(f)}$ where $\phi^{\prime}$ is a composite operator. E.g. $\phi^{\prime}=: \phi^{n}$ :, $n<\operatorname{deg} P$, in $P(\phi)_{2}$ is treated in [88]. See also [89] for the general problem to construct $: \phi^{2}$ : as a random distribution given $\phi$.

\subsubsection{Analytic continuation}

There are three tricks used together to construct the analytic continuation of $S_{n}$. The first trick was already used above: it is the observation that if the states $\left|\Psi_{n}\left(t ; y_{1}, \ldots, y_{n}\right)\right\rangle$ and $\left|\Psi_{m}\left(t^{\prime} ; y_{1}^{\prime}, \ldots, y_{m}^{\prime}\right)\right\rangle$ are defined for some values of $t, y_{k}$ and $t^{\prime}, y_{k}^{\prime}$, then we can compute the matrix elements

$$
\begin{aligned}
& \left\langle\Psi_{m}\left(t^{\prime} ; y_{1}^{\prime} \ldots y_{m-1}^{\prime}\right)\left|U_{\tau}\right| \Psi_{n}\left(t ; y_{1} \ldots y_{n-1}\right)\right\rangle \\
& =S_{m+n-1}\left(\overline{y_{m-1}^{\prime}}, \ldots, \overline{y_{1}^{\prime}}, t^{\prime}+t+\tau, y_{1}, \ldots, y_{n-1}\right)
\end{aligned}
$$

with $\operatorname{Re} \tau>0$, thus potentially extending the domain of analyticity of $S_{m+n-1}$.

\footnotetext{
${ }^{79}$ Then $G_{n}^{E}\left(f_{1} \otimes \ldots \otimes f_{n}\right)$ is a sum of $(n-1) ! !$ terms, products of Wick contractions $G_{2}\left(f_{i} \otimes f_{j}\right)$, which can be bounded by $A\left|f_{i}\right|_{r}\left|f_{j}\right|_{r}$ where $r$ depends on the UV dimension of $\mathcal{O}$. We thus get (9.19) with $\sigma_{n}=(n-1) ! ! A^{n / 2}$.

${ }^{80}$ See e.g. [87], theorem 1.1.8, which establishes eq. (9.19) for Schwinger functions of arbitrarily high normal-ordered powers : $\phi^{n}$ : of the fundamental field $\phi$ in weakly coupled $P(\phi)_{2}$ theories.

${ }^{81}$ See [88], section 6. This book introduced axioms for random distributions, numbered OS0-OS5. This chosen name is a bit unfortunate because these axioms are quite different in spirit from the original Osterwalder-Schrader axioms described in section 2.2, and appear much stronger. E.g. they make the recovery of Wightman axioms a relatively trivial task. We don't know how to derive the axioms of [88] from the Euclidean CFT axioms.
} 
The second trick, intuitively, says that we can write

$$
\left\langle\Psi_{n}\left(t ; y_{1} \ldots y_{n-1}\right) \mid \Psi_{n}\left(t ; y_{1} \ldots y_{n-1}\right)\right\rangle=S_{2 n-1}\left(\overline{y_{n-1}}, \ldots, \overline{y_{1}}, 2 t, y_{1}, \ldots, y_{n-1}\right),
$$

and so the state $\left|\Psi_{n}\left(t ; y_{1} \ldots y_{n-1}\right)\right\rangle$, whose norm appears in the left-hand side, should be well-defined as long as the correlation function in the right-hand side is well-defined. That is, while we start with the states $\left|\Psi_{n}\left(t ; y_{1} \ldots y_{n-1}\right)\right\rangle$ defined for positive real $y_{k}$, we should be able to analytically continue them in $y_{k}$ if we manage to analytically continue the correlators $S_{2 n-1}$. Of course, this is not a proof that $\left|\Psi_{n}\left(t ; y_{1} \ldots y_{n-1}\right)\right\rangle$ is well-defined. We will give the proof below, after we get more information about the domain in which we wish to construct it.

The final trick is the idea of analytic completion for functions of several complex variables. Recall that for $n>1$ not every domain in $\mathbb{C}^{n}$ is the domain of holomorphy of some holomorphic function: there exist domains $\mathcal{D} \subset \mathbb{C}^{n}$ such that any $f$ holomorphic in $\mathcal{D}$ can be extended to a function holomorphic in a strictly larger domain $\mathcal{D}^{\prime} \supset \mathcal{D}$. For our applications the relevant theorem is Bochner's tube theorem, which states that any holomorphic function in a tube domain of the form $\mathcal{D}=\mathbb{R}^{n}+i X$, where $X$ is a connected open subset of $\mathbb{R}^{n}$, can be extended to a holomorphic function on $\mathcal{D}^{\prime}=\operatorname{ch}(\mathcal{D})=\mathbb{R}^{n}+i \operatorname{ch}(X)$, where ch denotes the convex hull. Note that since $\mathcal{D}^{\prime}$ is a convex set, it is a domain of holomorphy ${ }^{82}$ and so $f$ cannot be extended any further by analytic completion alone. The requirement that $X$ is open is a bit too restrictive and we'll need also a degenerate case of this theorem, as described below.

These three tricks are applied one by one infinitely many times in order to construct the full analytic continuation of $S_{n}$. Instead of setting up the procedure in its full glory, we will only follow the first steps to see how it works in principle. The full analysis is performed in [3].

First, it helps to introduce new variables $w_{i}$ by

$$
e^{w_{i}}=y_{i}
$$

Our domains of analyticity in terms of $w_{i}$ will always be tubes of the form $\left(w_{1} \ldots w_{n}\right) \in$ $\mathcal{D}(X) \equiv \mathbb{R}^{n}+i X$ for various $X \subset \mathbb{R}^{n}$, and so we'll often just describe $X$. For example, we start with $S_{n}$ and $\Psi_{n}$ defined for real positive $y_{i}$, which corresponds to the domain $\mathcal{D}(\{0\})$ in $w_{i}$.

Consider the 2-point function $S_{1}\left(y_{1}\right)$. We start with the domain $\mathcal{D}(\{0\})=\mathbb{R}$ in $w_{1}$, corresponding to real positive $y_{1}$. Next, we apply the first trick. Specifically, we write

$$
\left\langle\Psi_{1}\left(t^{\prime}\right)\left|U_{\tau}\right| \Psi_{1}(t)\right\rangle=S_{1}\left(t^{\prime}+t+\tau\right)
$$

\footnotetext{
${ }^{82}$ To see this, it suffices to show that for any point $z$ on the boundary of $\mathcal{D}^{\prime}$ there exists a function holomorphic in $\mathcal{D}^{\prime}$ but singular at $z$. In general, such functions might not exist since the set of singularities of a holomorphic function cannot be arbitrary. However, it is easy to construct a function singular on any complex codimension-1 hyperplane in $\mathbb{C}^{n}$ (take the reciprocal of an affine-linear function). For a convex $\mathcal{D}^{\prime}$ one can always find such a hyperplane passing through a given boundary point but staying away from the interior of $\mathcal{D}^{\prime}$.
} 


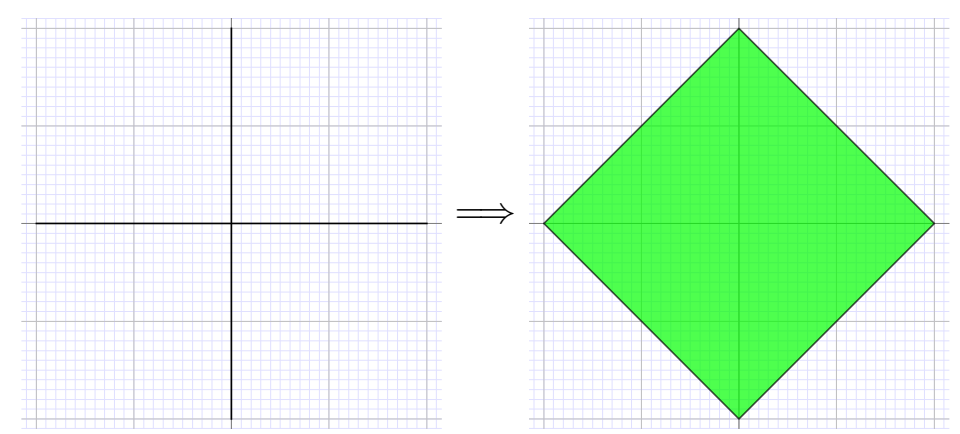

Figure 7. Left: set $X_{2}$. Right: domain $X_{2}^{\prime}$ which defines the envelope of holomorphy $\mathcal{D}\left(X_{2}^{\prime}\right)$ of $\mathcal{D}\left(X_{2}\right)$.

and since we are free to choose $t>0$ and $t^{\prime}>0$ arbitrarily small, while $U_{\tau}$, as discussed above, is a well-defined bounded operator for $\operatorname{Re} \tau>0$, we obtain an analytic continuation of $S_{1}\left(y_{1}\right)$ to the right half-plane.

We are now done with the analytic continuation of $S_{1}$, since our goal was to continue all $y_{k}$ to the right-half plane. In terms of $w_{i}$, this corresponds to the domain $w_{1} \in \mathcal{D}\left(\left(-\frac{\pi}{2},+\frac{\pi}{2}\right)\right)$, i.e. a strip. For higher-point functions, in terms of $w_{i}$, we should stop when our domain of analyticity is $\mathcal{D}\left(\left(-\frac{\pi}{2},+\frac{\pi}{2}\right) \times \cdots \times\left(-\frac{\pi}{2},+\frac{\pi}{2}\right)\right)$.

Consider now the 3-point function $S_{2}\left(y_{1}, y_{2}\right)$. We can again use the first trick and define it on $\left(w_{1}, w_{2}\right) \in \mathcal{D}\left(X_{2}\right)$, where $X_{2}=\{0\} \times\left(-\frac{\pi}{2},+\frac{\pi}{2}\right) \cup\left(-\frac{\pi}{2},+\frac{\pi}{2}\right) \times\{0\}$ (see figure 7 , left). In more detail, we write the following two equations for $S_{2}\left(y_{1}, y_{2}\right)$, representing it as an inner product in two ways, and inserting a $U_{\tau}$,

$$
\begin{aligned}
& \left\langle\Psi_{2}\left(t^{\prime} ; y_{1}^{\prime}\right)\left|U_{\tau}\right| \Psi_{1}(t)\right\rangle=S_{2}\left(y_{1}^{\prime}, t^{\prime}+t+\tau\right), \\
& \left\langle\Psi_{1}\left(t^{\prime}\right)\left|U_{\tau}\right| \Psi_{2}\left(t, y_{1}\right)\right\rangle=S_{2}\left(t^{\prime}+t+\tau, y_{1}\right),
\end{aligned}
$$

where the left-hand sides are well-defined (at this point) for $t, t^{\prime}, y_{1}, y_{1}^{\prime}>0$ and $\operatorname{Re} \tau>0$. We see that the first equation defines $S_{2}\left(y_{1}, y_{2}\right)$ for real $y_{1}>0$ and Re $y_{2}>0$ as a holomorphic function of $y_{2}$. The second equation does the same, but with $y_{1}$ and $y_{2}$ exchanged. In terms of $\left(w_{1}, w_{2}\right)$ this corresponds to the "analyticity domain" $\mathcal{D}\left(X_{2}\right)$ described above. We write "analyticity domain" in quotes because $\mathcal{D}\left(X_{2}\right)$ is not open (and has empty interior), and thus is not a domain. Correspondingly, we cannot say that $S_{2}$ is an holomorphic function of two variables on $\mathcal{D}\left(X_{2}\right)$. We will deal with this problem momentarily.

To proceed with the analytic continuation of $S_{2}\left(y_{1}, y_{2}\right)$, we want to use the third trick, the tube theorem, to extend the analyticity domain from $\mathcal{D}\left(X_{2}\right)$ to $\mathcal{D}\left(X_{2}^{\prime}\right)$, with $X_{2}^{\prime} \equiv \operatorname{ch}\left(X_{2}\right)$ (figure 7, right).

The problem with this is that $X_{2}$ is not open, as mentioned above, so the tube theorem does not apply. Instead, for this step one has to use Malgrange-Zerner theorem [90], which allows $X_{2}$ to be a union of intervals, with $S_{2}\left(y_{1}, y_{2}\right)$ separately holomorphic in one variable on each of these intervals, as is the case in our setup. The conclusion is still that $S_{2}\left(y_{1}, y_{2}\right)$ can be analytically continued to $\mathcal{D}\left(X_{2}^{\prime}\right)$.

Note that the domain $\mathcal{D}\left(X_{2}^{\prime}\right)$ is not yet the full analyticity domain $\mathcal{D}\left(\left(-\frac{\pi}{2},+\frac{\pi}{2}\right) \times\left(-\frac{\pi}{2},+\frac{\pi}{2}\right)\right)$ that we are aiming for. In particular, $X_{2}^{\prime}$ is a proper 
subset of the square $\left(-\frac{\pi}{2},+\frac{\pi}{2}\right) \times\left(-\frac{\pi}{2},+\frac{\pi}{2}\right)$, see the right panel of figure 7 . Importantly, it doesn't approach the corners $\left( \pm \frac{\pi}{2}, \pm \frac{\pi}{2}\right)$, which correspond to pure imaginary $y_{1}, y_{2}$. Pure imaginary $y_{1}, y_{2}$ is, in turn, where we want to recover the Wightman distributions.

To extend the domain of analyticity of $S_{2}\left(y_{1}, y_{2}\right)$ even further, we need to first extend the domain of $\Psi_{2}\left(t, y_{1}\right)$, which can be done by the second trick above - via the equality

$$
\left\langle\Psi_{2}\left(t, y_{1}\right) \mid \Psi_{2}\left(t, y_{1}\right)\right\rangle=S_{3}\left(\overline{y_{1}}, 2 t, y_{1}\right) .
$$

Note that we are not interested in the analytic continuation in $t$ here - it is automatic when we act on $\Psi_{2}$ with $e^{-H t}$ - so we can assume $t$ is real. For $S_{3}$ we can run the same argument as we just did for $S_{2}$ and conclude that it is holomorphic in $\mathcal{D}\left(X_{3}^{\prime}\right)$, where $X_{3}^{\prime}$ is the convex hull of three intersecting intervals on coordinate axes (an octahedron). As discussed above, we expect that $\Psi_{2}\left(t, y_{1}\right)$ is defined whenever $t$ and $y_{1}$ are such that the arguments of $S_{3}$ above are in its analyticity domain. This happens whenever

$$
\left(\overline{w_{1}}, \log 2 t, w_{1}\right) \in \mathcal{D}\left(X_{3}^{\prime}\right)
$$

which is equivalent to

$$
\left(\operatorname{Im} \overline{w_{1}}, \operatorname{Im} \log 2 t, \operatorname{Im} w_{1}\right) \in X_{3}^{\prime} .
$$

Since we take $t$ to be real and positive, we have $\operatorname{Im} \log 2 t=0$ and so $t$ is otherwise unconstrained. By construction of $X_{3}^{\prime}$ and $X_{2}^{\prime},\left(\operatorname{Im} \overline{w_{1}}, 0, \operatorname{Im} w_{1}\right) \in X_{3}^{\prime}$ is equivalent to $\left(\operatorname{Im} \bar{w}_{1}, \operatorname{Im} w_{1}\right) \in X_{2}^{\prime}$. Using $\operatorname{Im} \overline{w_{1}}=-\operatorname{Im} w_{1}$, we conclude that $w_{1}$ is constrained by

$$
\left(-\operatorname{Im} w_{1}, \operatorname{Im} w_{1}\right) \in X_{2}^{\prime}
$$

This is equivalent to $\left|\operatorname{Im} w_{1}\right|<\frac{\pi}{4}$, which is the same as $w_{1} \in \mathcal{D}\left(\left(-\frac{\pi}{4},+\frac{\pi}{4}\right)\right)$. To conclude, we expect $\Psi_{2}\left(t, y_{1}\right)$ to be defined and holomorphic in $y_{1}$ for $t>0$ and $w_{1} \in \mathcal{D}\left(\left(-\frac{\pi}{4},+\frac{\pi}{4}\right)\right)$.

We can now apply the first trick to $S_{2}\left(y_{1}, y_{2}\right)$ again, writing it as inner product of $\Psi_{1}$ and $\Psi_{2}$ in the two ways (9.25) and (9.26). However, this time we can use $\Psi_{2}\left(t, y_{1}\right)$ in a wider domain of $y_{1}$, as computed above, equivalent to $w_{1} \in \mathcal{D}\left(\left(-\frac{\pi}{4},+\frac{\pi}{4}\right)\right)$. From (9.25) we conclude that $S_{2}\left(y_{1}, y_{2}\right)$ is analytic for

$$
\left(w_{1}, w_{2}\right) \in \mathcal{D}\left(\left(-\frac{\pi}{4},+\frac{\pi}{4}\right) \times\left(-\frac{\pi}{2},+\frac{\pi}{2}\right)\right),
$$

where the domain of analyticity in $w_{1}$ comes from that of $\Psi_{2}\left(t, y_{1}\right)$, and in $w_{2}$ from $e^{-H \tau}$. Similarly, (9.26) now implies analyticity in the domain

$$
\left(w_{1}, w_{2}\right) \in \mathcal{D}\left(\left(-\frac{\pi}{2},+\frac{\pi}{2}\right) \times\left(-\frac{\pi}{4},+\frac{\pi}{4}\right)\right) .
$$

Combining the two together, we find that $S_{2}\left(y_{1}, y_{2}\right)$ is analytic for $\left(w_{1}, w_{2}\right) \in \mathcal{D}\left(X_{2}^{\prime \prime}\right)$, where

$$
X_{2}^{\prime \prime} \equiv\left(-\frac{\pi}{4},+\frac{\pi}{4}\right) \times\left(-\frac{\pi}{2},+\frac{\pi}{2}\right) \cup\left(-\frac{\pi}{2},+\frac{\pi}{2}\right) \times\left(-\frac{\pi}{4},+\frac{\pi}{4}\right),
$$

see the left panel of figure 8 . 


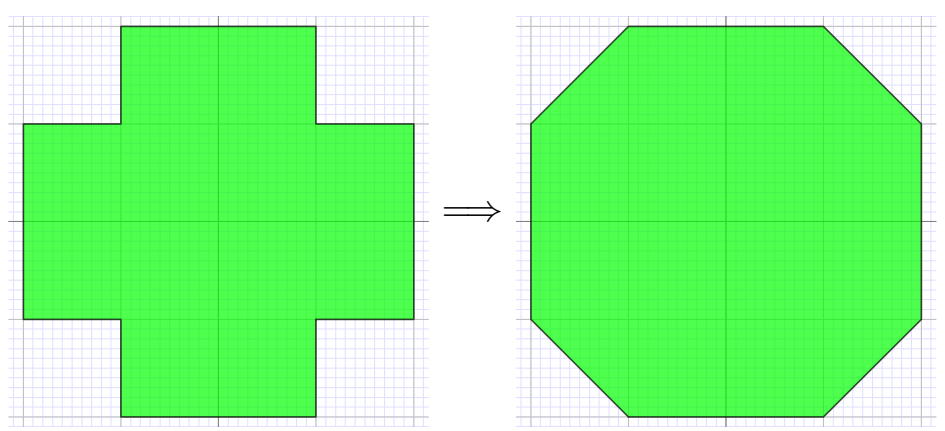

Figure 8. Left: set $X_{2}^{\prime \prime}$. Right: domain $X_{2}^{\prime \prime \prime}$ which defines the envelope of holomorphy $\mathcal{D}\left(X_{2}^{\prime \prime \prime}\right)$ of $\mathcal{D}\left(X_{2}^{\prime \prime}\right)$.

Using the tube theorem, we can now extend the analyticity domain from $\mathcal{D}\left(X_{2}^{\prime \prime}\right)$ further to $\mathcal{D}\left(X_{2}^{\prime \prime \prime}\right)$, where $X_{2}^{\prime \prime \prime} \equiv \operatorname{ch}\left(X_{2}^{\prime \prime}\right)$ is the convex hull of $X_{2}^{\prime \prime}$ shown in the right panel of figure 8 .

We see that in order to analytically continue the 3-point function $S_{2}$, it was useful to split it into an inner product of one-operator and two-operator states $\Psi_{1}$ and $\Psi_{2}$, and use the information about the latter that is provided by its norm, the 4-point function $S_{3}$. Still, we have not yet managed to analytically continue $S_{2}$ to the entire region of interest (we still have the corners missing in the right panel of figure 8). The only way to fix this is to extend the region of analyticity of $S_{3}$. For that, we have to split it into a product of two states, and extend the region of analyticity of these states. It is useless to split it as a product of two $\Psi_{2}$ states, since their norm is computed by $S_{3}$ itself and we won't learn anything new in this way. Instead, we have to split it as a product of $\Psi_{1}$ and $\Psi_{3}$. This will lead us to consider the norm of $\Psi_{3}$, which is computed by the six-point function $S_{5}$. Following this logic, eventually, we will be forced to consider $S_{n}$ with arbitrarily high $n$ just in order to construct the analytic continuation of $S_{2}$. Fortunately, it can be shown that this procedure converges to the desired domain $\mathcal{D}\left(\left(-\frac{\pi}{2},+\frac{\pi}{2}\right) \times \cdots \times\left(-\frac{\pi}{2},+\frac{\pi}{2}\right)\right)$ for all $S_{n}$, see [3] for details.

To finish the discussion of the analytic continuation of $S_{n}$, let us justify the second trick, which constructs the states $\Psi_{n}$ based on analyticity of their norm $S_{2 n-1}$. Let $C$ be the domain of analyticity of $S_{2 n-1}\left(y_{1} \ldots y_{2 n-1}\right)$ known to us, expressed in terms of $w_{i}$, and let $D$ be the domain of the arguments $t, w_{1} \ldots w_{n-1}$ of $\Psi_{n}\left(t ; y_{1} \ldots y_{n-1}\right)$ for which the arguments of $S_{2 n-1}$ in the right-hand side of

$$
\left\langle\Psi_{n}\left(t ; y_{1} \ldots y_{n-1}\right) \mid \Psi_{n}\left(t ; y_{1} \ldots y_{n-1}\right)\right\rangle=S_{2 n-1}\left(\overline{y_{n-1}}, \ldots, \overline{y_{1}}, 2 t, y_{1}, \ldots, y_{n-1}\right),
$$

belong to $C$. As is clear from the above discussion, $C$ (expressed in terms of $w_{i}$ ) is always of the form $C=\mathcal{D}(X)$ for some $X$. We similarly have $D=\mathcal{D}(Y)$ for some $Y$. By the tube theorem (or Malgrange-Zerner theorem), we can assume that $X$ (and thus also $Y$ ) is open, non-empty, and convex. Furthermore, it is easy to convince oneself that $X$, and thus $Y$, is invariant under reflections along any of the coordinate real axes (i.e. sending $w_{i}$ to $\overline{w_{i}}$ for some $i$ ).

Suppose now that we have a point $\left(t ; w_{1}^{0} \ldots w_{n-1}^{0}\right) \in D$. Then by definition of $D$ we have

$$
p \equiv\left(\overline{w_{n-1}^{0}} \ldots \overline{w_{1}^{0}}, \log 2 t, w_{1}^{0} \ldots w_{n-1}^{0}\right) \in C .
$$




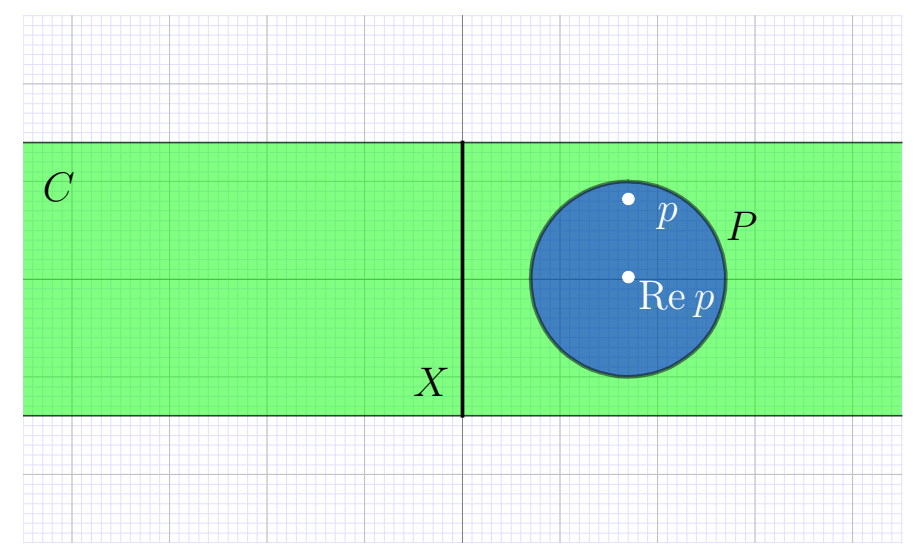

Figure 9. Schematic picture of the tube $C$ and polydisk $P$.

The above properties imply that there are $r_{i}>0$ such that the polydisk

$$
P=\left\{\left(w_{1} \ldots w_{2 n-1}\right)|| w_{i} \mid<r_{i}\right\}+\operatorname{Re} p
$$

is contained in $C, P \subset C$, and moreover $p \in P$. Indeed, since $C=\mathcal{D}(X)$, this will be true if $\operatorname{Im} P \subset X$ and $\operatorname{Im} p \in \operatorname{Im} P .{ }^{83}$ By construction, $\operatorname{Im} P$ is a box with sides $2 r_{i}$ centered at 0 . On the other hand, the properties of $X$ imply that together with any point $x, X$ contains such a box with $x$ being one of its vertices. We can then find an $\varepsilon>0$ such that $(1+\varepsilon) \operatorname{Im} p \in X$, and take $\operatorname{Im} P$ to be the box defined by the vertex $x=(1+\varepsilon) \operatorname{Im} p$. See figure 9 for an intuitive picture.

Writing temporarily the state $\Psi_{n}$ as a function of $w_{k}$ instead of $y_{k}$, we define it at $w_{k}$ by the Taylor series

$$
\left|\Psi_{n}\left(t ; w_{1} \ldots w_{n-1}\right)\right\rangle \equiv \sum_{\alpha} \frac{\left(w-\operatorname{Re} w^{0}\right)^{\alpha}}{\alpha !} \partial^{\alpha}\left|\Psi_{n}\left(t ; \operatorname{Re} w_{1}^{0} \ldots \operatorname{Re} w_{n-1}^{0}\right)\right\rangle
$$

( $\alpha$ is a multiindex so $w^{\alpha}=w_{1}^{\alpha_{1}} w_{2}^{\alpha_{2}} \ldots$ etc.). Note that the state in the right-hand side is well defined since the corresponding $y_{k}=e^{\operatorname{Re} w_{k}^{0}}>0$. To check whether this Taylor series converges, we look at its remainder

$$
\sum_{|\alpha|>N} \frac{\left(w-\operatorname{Re} w^{0}\right)^{\alpha}}{\alpha !} \partial^{\alpha}\left|\Psi_{n}\left(t ; \operatorname{Re} w_{1}^{0} \ldots \operatorname{Re} w_{n-1}^{0}\right)\right\rangle,
$$

whose norm squared is

$$
\begin{aligned}
& \sum_{|\alpha|>N} \sum_{|\beta|>N} \frac{\left(w-\operatorname{Re} w^{0}\right)^{\alpha}}{\alpha !} \frac{\left(\bar{w}-\operatorname{Re} w^{0}\right)^{\beta}}{\beta !} \\
& \quad \times \partial^{\alpha} \partial^{\beta} S_{2 n-1}\left(\operatorname{Re} w_{n-1}^{0} \ldots \operatorname{Re} w_{1}^{0}, \log 2 t, \operatorname{Re} w_{1}^{0} \ldots \operatorname{Re} w_{n-1}^{0}\right),
\end{aligned}
$$

where $\beta$-derivatives act on the first $n-1$ arguments of $S_{2 n-1}$, while $\alpha$-derivatives act on the last $n-1$ arguments. Here we also temporarily write $S_{2 n-1}$ as function of $w_{k}$. This

\footnotetext{
${ }^{83}$ The latter is because $\operatorname{Im}$ of the section of $P$ by $\operatorname{Re} x=\operatorname{Re} p$ is $\operatorname{Im} P$.
} 
norm is clearly just the tail of the Taylor series of $S_{2 n-1}$ expanded around the point $\operatorname{Re} p$, and evaluated at $\left(\overline{w_{n-1}}, \ldots, \overline{w_{1}}, \log 2 t, w_{1}, \ldots, w_{n-1}\right)$. (We are not expanding in $t$.) Since $S_{2 n-1}$ is holomorphic in the polydisk $P$ centered at $\operatorname{Re} p$, this Taylor series converges in $P$ and thus this remainder tends to 0 there.

Since $p=\left(\overline{w_{n-1}^{0}} \ldots \overline{w_{1}^{0}}, \log 2 t, w_{1}^{0} \ldots w_{n-1}^{0}\right) \in P$, the remainder tends to 0 at $p$, and thus (9.36) converges at $\left(t ; w_{1}^{0} \ldots w_{n-1}^{0}\right)$. Furthermore, since $P$ is open, it follows that (9.36) converges in some neighborhood of $\left(t ; w_{1}^{0} \ldots w_{n-1}^{0}\right)$, defining $\left|\Psi_{n}\left(t ; w_{1} \ldots w_{n-1}\right)\right\rangle$ as a holomorphic $\mathcal{H}^{\mathrm{OS}}$-valued function in that neighborhood. Since the choice of $\left(t ; w_{1}^{0} \ldots w_{n-1}^{0}\right) \in D$ was arbitrary, we have defined $\left|\Psi_{n}\left(t ; w_{1} \ldots w_{n-1}\right)\right\rangle$ as a holomorphic function of $w_{i}$ for all points in $D$.

\subsubsection{Temperedness bound}

Now that the correlation functions $S_{n}\left(y_{1} \ldots y_{n}\right)$ have been analytically continued from $y_{k}>0$ to $\operatorname{Re} y_{k}>0$, we only need to establish a bound on their growth as $\operatorname{Re} y_{i} \rightarrow 0$ in order to construct tempered Wightman distributions by an application of Vladimirov's theorem. The logic proceeds by establishing a bound on $S_{n}\left(y_{1} \ldots y_{n}\right)$ for real $y_{k}$, and then repeating the analytic continuation described above, while keeping track of this bound. We will only sketch this rather technical argument in very general terms.

The final temperedness bound that we want to establish is

$$
\left|S_{n}\left(y_{1} \ldots y_{n}\right)\right| \leqslant c_{n}\left(\left(1+\sum_{k}\left|y_{k}\right|\right)\left(1+\sum_{k}\left(\operatorname{Re} y_{k}\right)^{-1}\right)\right)^{p_{n}},
$$

for some sequences $c_{n}$ and $p_{n} .{ }^{84}$ We would like (9.39) to hold for all $y_{k}$, Re $y_{k}>0$. For real positive $y_{k}$ (i.e. in the Euclidean) this holds as a consequence of (2.15). As discussed in remark 2.4, the original OS axioms did not include (2.15), so their first step was to derive (9.39) for $y_{k}>0$ using (9.17).

In principle at fixed $n,(9.39)$ looks reasonable given (9.17): both say, intuitively, that the correlation functions cannot be too singular at coincident points or grow too fast at infinity. However, (9.39) imposes this in a much more direct way. It turns out that in general one cannot derive direct bounds such as (9.39) from averaged statements such as (9.17), even if we know that $S_{n}$ is real analytic.

Consider the real-analytic function $\sin \left(e^{x}\right), x \in \mathbb{R}$. It is a bounded function, hence a tempered distribution. Thus its first derivative $e^{x} \cos \left(e^{x}\right)$ is also a tempered distribution. This is an example of a real-analytic tempered distribution which is not polynomially bounded. So some further assumptions are needed beyond real analyticity. ${ }^{85}$

In our case, the functions $S_{n}\left(y_{1} \ldots y_{n}\right)$ are real-analytic and satisfy (9.17). In addition, they satisfy OS positivity. We already used OS positivity to show real-analyticity, and we will now have to invoke it again to prove (9.39) for $y_{k}>0$. The full argument is rather

\footnotetext{
${ }^{84}$ Here, for simplicity, we again ignore spatial arguments of the correlation functions, although they need to be taken care of at this step in order to establish "temperedness in spatial directions." Furthermore, note that Osterwalder and Schrader establish additional bounds on $c_{k}$, etc., which are not important for the application of Vladimirov's theorem.

${ }^{85}$ Incidentally, our example shows that the Corollary of lemma 1 in [46] is wrong.
} 
technical; we will explain the main idea on the example of $S_{1}(y)$. Since we know that $S_{1}$ is holomorphic, in particular harmonic, by the mean value theorem for harmonic functions we can write it as a radially symmetric average

$$
\begin{aligned}
S_{1}(y) & =\int d x d t S_{1}(y+x+i t) k_{\rho}(x, t) \\
& =\int_{|t|,\left|t^{\prime}\right|<\rho} d t d t^{\prime} T\left(t \mid g_{\rho}\left(\cdot, t+t^{\prime}\right), g_{\rho}\left(\cdot, t^{\prime}\right)\right), \\
T\left(t \mid \varphi_{1}, \varphi_{2}\right) & :=\int d x d x^{\prime} S_{1}(y+x+i t) \varphi_{1}\left(x+x^{\prime}\right) \varphi_{2}\left(x^{\prime}\right),
\end{aligned}
$$

where $k_{\rho}$ is a $C_{0}^{\infty}$ radial function supported in a ball of radius $\rho$ and of integral 1 , and we choose $\rho$ sufficiently small so that all points under the integral sign are where $S_{2}$ is analytic. We also chose

$$
k_{\rho}(x, t)=\int d x^{\prime} d t^{\prime} g_{\rho}\left(x+x^{\prime}, t+t^{\prime}\right) g_{\rho}\left(x^{\prime}, t^{\prime}\right),
$$

a convolution of another radial $C_{0}^{\infty}$ function with itself (and hence a radial function). The point of this construction is that, for generic $\varphi_{1}, \varphi_{2}, T\left(0 \mid \varphi_{1}, \varphi_{2}\right)$ is an inner product $\left\langle\Psi_{1} \mid \Psi_{2}\right\rangle$ of two OS states:

$$
\left\langle\Psi_{1}\left|=\int d x \mathcal{O}(y / 2+x) \varphi_{1}(x), \quad\right| \Psi_{2}\right\rangle=\int d x \mathcal{O}(-y / 2+x) \varphi_{2}(x) .
$$

The norm of these states, and hence their inner product, can be bounded using (9.17). Furthermore $T\left(t \mid \varphi_{1}, \varphi_{2}\right)=\left\langle\Psi_{1}\left|e^{-i H t}\right| \Psi_{2}\right\rangle$ satisfies the same bound. Using this bound for $\varphi_{1}=g_{\rho}\left(\cdot, t+t^{\prime}\right), \varphi_{2}=g_{\rho}\left(\cdot, t^{\prime}\right)$, eq. (9.40) gives a bound on $S_{1}(y)$. The same idea works for higher point functions. We first have to estimate the norm of some states using (9.17). ${ }^{86} \mathrm{We}$ then analytically continue separately in each time, and then use Malgrange-Zerner theorem to extend the bound on $T$ to an open set. A single use of Malgrange-Zerner theorem suffices here, like in figure 7 . We refer the reader to section VI.1 of [3] for full details.

Once (9.39) is established for $y_{k}>0$, one repeats the analytic continuation procedure that we described above, keeping track of the implications of (9.39). The analytic continuation used three tricks: (1) analytically continuing $S_{n}$ by representing it in the form (9.21) (as $e^{-H \tau}$ inserted between two states), (2) expressing the norms of these states in terms of higher-point $S_{n}$ as in (9.22), and (3) analytic completion.

The bound (9.39) propagates through the tricks (1) and (2) by the use of CauchySchwarz inequality, as well as by using the fact that the norm of $e^{-H \tau}$ is bounded from above by 1 (i.e. eq. (9.6)).

To propagate the bound through trick (3), the following simple idea is used. Suppose we have domains $\mathcal{D}^{\prime} \supset \mathcal{D}$ such that any holomorphic function $f$ on $\mathcal{D}$ can be extended to a holomorphic function on $\mathcal{D}^{\prime}$. Then we have the equality of images

$$
f\left(\mathcal{D}^{\prime}\right)=f(\mathcal{D})
$$

\footnotetext{
${ }^{86}$ Note that the linear growth condition is not needed at this point: eq. (9.17) with some $\sigma_{n}$ and $q_{n}$ suffices to establish (9.39) with some $c_{n}$ and $p_{n}$. The linear growth condition gives in addition $c_{n}$ of factorial growth and $p_{n}$ growing at most linearly. This turns out important later in the proof, see below.
} 
and in particular

$$
\sup _{z \in \mathcal{D}^{\prime}}|f(z)|=\sup _{z \in \mathcal{D}}|f(z)|
$$

To see this, suppose $a \in \mathbb{C}$ is a value which $f$ assumes in $\mathcal{D}^{\prime}$ but not in $\mathcal{D}$. Then the function $(f(z)-a)^{-1}$ is holomorphic in $\mathcal{D}$ but has a singularity in $\mathcal{D}^{\prime}$, which is a contradiction. This shows that if we have a bound on $f$ in $\mathcal{D}$, it is also valid in $\mathcal{D}^{\prime}$.

Finally, recall that in order to construct the analytic continuation of $S_{n_{0}}$ for some fixed $n_{0}$, we had to use $S_{n}$ with arbitrarily high $n$ in the process. This means that in order to establish the bound (9.39) on $S_{n_{0}}$ for all Re $y_{k}$, we have to use (9.39) for $y_{k}>0$ for $S_{n}$ with arbitrarily high $n$. These bounds need to combine in a way that is strong enough to establish (9.39) for $S_{n_{0}}$. For this, it is important that $c_{n}$ is of factorial growth and $p_{n}$ grows at most linearly. This requires the same of the sequence $\sigma_{n}$ and the index of the seminorm in (9.17), explaining the need for the linear growth condition.

\section{Conclusions}

In this paper we studied the relationship between the modern Euclidean CFT axioms (which we formulated in section 2.3) and the more traditional Osterwalder-Schrader and Wightman axioms. We showed that at least for $(n \leqslant 4)$-point functions, both OS and Wightman axioms follow from the Euclidean CFT axioms. Our Euclidean CFT axioms are quite modest. In particular, beyond the minimal assumptions of regularity of correlators and the standard constraints of unitarity, we assumed only a very weak form of the convergent OPE.

Our derivation of Wightman axioms is of particular importance: it shows that the conformal Wightman 4-point functions are well-defined tempered distributions for arbitrary configurations of the 4 points, even when no OPE channel is convergent in the sense of functions. We have furthermore shown that these tempered distributions can always be computed by a conformal block expansion which is convergent in the sense of distributions, generalizing our previous results in cross-ratio space [36], and giving a derivation of Mack's results [41] from Euclidean CFT axioms.

For example, consider the configuration in figure 10, where the operators in a 4-point function are inserted at $x_{1}=0, x_{3}=\widehat{e}_{1}, x_{4}=\infty \widehat{e}_{1}$, while $x_{2}=t \widehat{e}_{0}+x \widehat{e}_{1}$ is allowed to move in a plane parametrized by $(t, x)$. The cross-ratios for this configuration are $z, \bar{z}=x \pm t$. It is then easy to see that for $x_{2}$ in the blue region of figure $10|\rho|,|\bar{\rho}|<1$ and the s-channel OPE converges in the sense of functions. Our results imply that the s-channel OPE also converges in the red region where $|\rho|$ and/or $|\bar{\rho}|=1$, but now the convergence is in the sense of distributions. In particular, the 4-point function is at least a distribution for all values of $x_{2}$. Of course, in some regions of figure 10 this was obviously true - for example, in the red part of the regions $\mathbf{9 , 1 2}$ (labeling according to the classification in [37]), one can show that the 4-point function is real-analytic using the convergent t-channel OPE. One may hope to establish real-analyticity also in the region 7 using $\mathrm{u}$-channel OPE. This would indeed be the case for the ordering $\left\langle\mathcal{O}\left(x_{2}\right) \mathcal{O}\left(x_{1}\right) \mathcal{O}\left(x_{3}\right) \mathcal{O}\left(x_{4}\right)\right\rangle$. However, for the ordering $\left\langle\mathcal{O}\left(x_{1}\right) \mathcal{O}\left(x_{2}\right) \mathcal{O}\left(x_{3}\right) \mathcal{O}\left(x_{4}\right)\right\rangle$ that we are discussing here, it turns out that no OPE 


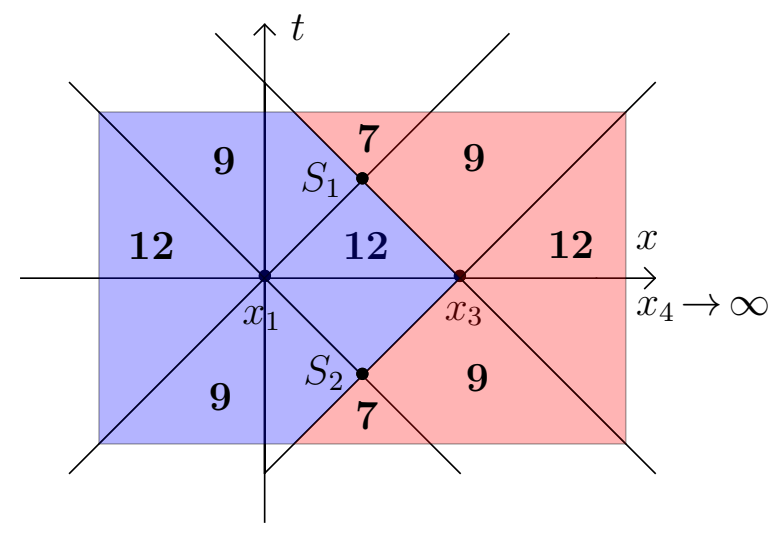

Figure 10. Minkowski configurations with $x_{1}=0, x_{3}=\widehat{e}_{1}, x_{4}=\infty \widehat{e}_{1}$ and $x_{2}=t \widehat{e}_{0}+x \widehat{e}_{1}$. Blue: configurations where $|\rho|,|\bar{\rho}|<1$ and the 4-point functions is analytic. Red: configurations where $|\rho|$ and/or $|\bar{\rho}|=1$. Boldface numbers $X=\mathbf{7 , 9 , 1 2}$ denote the causal type of the configuration according to [37] (excluding lightlike separations). $S_{1,2}$ are double light-cone singularities.

channel converges in region 7 in the sense of functions. ${ }^{87}$ Therefore, before our work it was not at all clear whether this correlator makes any sense in region 7 if we assume only the Euclidean CFT axioms.

While we have shown that the correlator is at least distributional in region 7 , we have not excluded the possibility of it being real-analytic there. For example, in 2 dimensions Virasoro symmetry implies that the 4-point function is analytic everywhere away from light-cone singularities [57]. This is perhaps too much to expect in higher dimensions, but one can still ask whether analyticity can be established in a larger domain. One approach is to ask for the envelope of holomorphy of the known domain of analyticity. Since the 4-point function is essentially only a function of two cross-ratios, this might be a tractable question [91]. We leave working out the full consequences of this idea for future work. ${ }^{88}$

In an upcoming paper [42], we will generalize our results to external operators with spins. In addition, there are many other fundamental open questions which we believe are important to understand. First of all, this paper is concerned with properties of CFT Wightman functions in Minkowski space. However, it is expected that Lorentzian CFTs should be naturally defined on Minkowski cylinder [71], which is the smallest physicallysensible space on which finite conformal transformations can act. Yet, it is not known whether CFT Wightman functions can be defined as tempered distributions on Minkowski

\footnotetext{
${ }^{87}$ For a reader comfortable with cuts in $z, \bar{z}$ plane the intuitive argument is simple: we have $z<0$ (on what we'll call s-channel cut), $\bar{z}>1$ (on t-channel cut). Furthermore according to the operator ordering, when $z$ crosses 0 we need to make $\operatorname{Im} t$ slightly negative (and thus $\operatorname{Im} z$ slightly negative), because $x_{2}$ at this point crosses the null cone of $x_{1}$, and when $\bar{z}$ crosses 1 we need to make $\operatorname{Im} t$ slightly positive (and thus $\operatorname{Im} \bar{z}$ slightly negative) because it corresponds to $x_{2}$ crossing the null cone of $x_{3}$. Thus both $z$ and $\bar{z}$ end on lower sides of their respective cuts, and so one of them must have crossed the $u$-channel cut at $(0,1)$ when analytically continuing from a Euclidean configuration. We conclude that s- and t- channel OPEs are only distributionally convergent, while $u$-channel is badly divergent.

${ }^{88}$ Another approach could be via alternative representations of the 4-point function having an extended region of analyticity, e.g. [92].
} 
cylinder (see note 68). Answering this question in the positive for CFT $(n \leqslant 4)$-point functions is the main goal of our forthcoming paper [72].

An important problem is to extend our results to $(n>4)$-point functions. As we discuss in appendix B, even deriving the OS axioms might require some strengthening of the OPE axiom. Another interesting possibility is to formulate Euclidean CFT axioms as OS axioms supplemented with a very weak form of the OPE (for example, asymptotic OPE in Euclidean space). This is perhaps less attractive, since it is desirable to formulate CFT axioms directly in terms of the CFT data (scaling dimensions and OPE coefficients). However, it will still be interesting to establish an equivalence between OS+(weak OPE axiom) and (possibly a stronger version of) our Euclidean CFT axioms, perhaps using arguments similar to those of [41]. Once OS axioms are established, it is likely that a strategy similar to that of the present paper can be pursued to establish Wightman axioms, using a comb-like OPE channel.

In this paper we only considered Wightman functions, but in practice one often needs time-ordered Minkowski correlators. Textbook definition of time-ordered correlators involves multiplying Wightman functions by $\theta$-functions implementing time ordering. Since Wightman functions are in general distributions, this definition does not make rigorous sense at coincident points. As a matter of fact, time-ordered correlators have not been rigorously defined just from Wightman axioms alone (see e.g. [77], p.505) in a general QFT. In a general QFT setting, it is known that defining time-ordered Minkowski correlators is closely related to defining Euclidean correlators at coincident points [93]. In the future, it would be interesting to define time-ordered CFT Minkowski correlators as distributions just from Euclidean CFT axioms. ${ }^{89}$

A more ambitious goal is to understand the relationship of CFT axioms to HaagKastler axioms. This appears to be considerably harder since these axioms deal with operator algebras rather than local correlation functions, and some qualitatively new ideas seem to be required.

\section{Acknowledgments}

Some of our results were first presented in a talk at the Simons Foundation [94], in lectures at the IPHT Saclay [69], and in an online seminar [95]. SR thanks Riccardo Guida and Antti Kupiainen for organizing the last two events.

SR thanks Gian Michele Graf for mentioning Zinoviev's work [85] and to David Brydges for the explanation of this work. We thank Marc Gillioz and Tom Hartman for communications concerning their work. We are grateful to Malek Abdesselam, Simon Caron-Huot, Tom Hartman and David Simmons-Duffin for comments on the draft.

PK is supported by DOE grant DE-SC0009988 and by the Adler Family Fund at the Institute for Advanced Study. The work of SR and JQ is supported by the Simons Foundation grant 488655 and 733758 (Simons Collaboration on the Nonperturbative Bootstrap). SR is supported by Mitsubishi Heavy Industries as an ENS-MHI Chair holder.

\footnotetext{
${ }^{89}$ Time-ordered Minkowski correlators are expected to be to some extent ambiguous at coincident points, just as the Euclidean correlators. For external CFT operators of non-integer external dimension one may choose to fix this ambiguity imposing scale invariance. Ambiguity will remain for external fields of integer dimensions, like the stress tensor.
} 


\section{A Lorentzian CFT literature}

Recent years have seen an explosion of the uses of Lorentzian CFT, motivated in particular by the conformal bootstrap applications. In this appendix we will mention some of these works, and comment on their underlying assumptions. See also [96] for a modern pedagogical introduction to Lorentzian CFT.

Conformal collider bounds. One of the first "modern" Lorentzian CFT results was obtained in [97]. This work considered a thought experiment, creating a CFT state via a (smeared) local operator and measuring energy coming out at null infinity in a particular direction, integrated over time. On physical grounds, one expects $\left\langle\Psi\left|\int d x^{-} T_{--}\right| \Psi\right\rangle \geqslant 0$ for any state ("averaged null energy condition" - ANEC). One interesting case is of 3-point functions $\left\langle\mathcal{O}^{\dagger} T_{\mu \nu} \mathcal{O}\right\rangle$ where $\mathcal{O}$ has nontrivial spin, when there are several independent OPE coefficients multiplying different tensor structures allowed by conformal symmetry. In this case ANEC implies that certain linear combinations of these OPE coefficients must be non-negative ("conformal collider bounds"). Interference effects can be used to strengthen conformal collider bounds to provide explicit lower bounds [98], while combining conformal collider bounds with stress-tensor Ward identities leads to constraints on operator dimensions which are sometimes stronger than standard unitarity bounds [99]. See below for work aiming to justify ANEC, or to derive conformal bounds directly without using ANEC.

Light-cone bootstrap. Refs. $[67,100]$ were the first to notice that some bootstrap constraints become more visible in the Lorentzian signature. These references pioneered the "analytic light-cone bootstrap" which studies conformal four point functions in the regime of $0<z, \bar{z}<1$ real, i.e. in the kinematics of figure 10 when the point $x_{2}$ is spacelike with respect to $x_{1}, x_{3}$. By studying the light cone limit $z \rightarrow 0$ at fixed $\bar{z}$ of one OPE channel and requiring that it should be reproduced by the crossed channel, they argued that, in any CFT for $d>2$, the OPE should contain a series of operators of arbitrarily large spin and twist asymptoting to a particular value. The original argument has some caveats (see the discussion in [101], appendix F) and a mathematically rigorous proof is lacking. It would be nice to provide such a proof, given the extreme importance of the light-cone bootstrap in the modern bootstrap literature. There is little doubt that the light-cone bootstrap results are correct. Numerical bootstrap studies of the critical $3 \mathrm{~d}$ Ising and the $O(2)$ models $[102,103]$ have found the series of operators predicted by the light-cone bootstrap see also [104]). Ref. [105] used the light-cone bootstrap to derive the conformal collider bounds of [97] without using ANEC.

Causality constraints. Refs. $[4,45,106]$ pioneered the study of causality constraints for CFT 4-point functions. In particular ref. [4] pointed out that the $z, \bar{z}$ and $\rho, \bar{\rho}$ expansions are sufficient to construct Lorentzian 4-point functions for many kinematic configurations and show local commutativity (i.e. that spacelike-separated operators commute). See also note 64. These techniques led to a proof of ANEC [45]. As mentioned in footnote 29, some steps in these papers are not completely rigorous. See appendix A.1 below for a more detailed review of [4]. 
Bulk point singularity. Ref. [57] studied the CFT 4-point function on the Lorentzian cylinder focusing on "bulk-point" configurations which correspond to scattering events in AdS/CFT [107-111]. Using a local AdS dual description, one may suspect that the 4-point function should be singular at such configurations. However, on the boundary CFT side, one does not see this singularity in perturbation theory in $d=2$ and $d=3$ dimensions [57]. In $d=2$, ref. [57] showed non-perturbatively that the CFT 4-point function is analytic everywhere away from light cones (in particular regular at bulk-point configurations). This assumes Virasoro symmetry and unitarity and uses Zamolodchikov's $q$-variables [112]. What happens non-perturbatively in $d \geqslant 3$ (or in $d=2$ in the absence of the local stress tensor) is still an open problem. Note that at bulk-point configurations, the $\rho$-expansion of the CFT 4-point function does not absolutely converge in s-channel (as $|\rho|=|\bar{\rho}|=1$ there) and diverges in t-,u-channels [37]. In this paper we only considered the CFT 4-point functions in flat space, but by the same strategy we will show in [72] that the Wightman axioms also hold for CFT 4-point functions on Lorentzian cylinder. In particular, this will show that the CFT 4-point functions are well defined at bulk-point configurations in the sense of tempered distributions (but it will not settle the question of their analyticity there).

Lorentzian inversion formula. Ref. [56] introduced an analogue of Froissart-Gribov formula in the context of conformal field theory, which is now known as the Lorentzian inversion formula (LIF). This formula computes the OPE data of a scalar 4-point function in terms of a Lorentzian integral of this 4-point function. The OPE data is extracted in the form of a function $C(\Delta, \ell)$. For integer $\ell$, the function $C(\Delta, \ell)$ encodes the scaling dimensions of exchanged primary operators of spin $\ell$ in the positions of poles in $\Delta$, and the corresponding OPE coefficients are encoded in residues. LIF has many interesting properties, such as analyticity in $\ell$, and suppression of double-twist operators when a crosschannel conformal block expansion is used under the integral. The original derivation of [56] was done in cross-ratio space. The formula was re-derived in position space in [113]. The derivation was further simplified and generalized in [55].

Among other applications, LIF has been used to systematize and extend many of the results of light-cone bootstrap (see, e.g., [103, 114-120]). Similarly to light-cone bootstrap, this application is not completely rigorous simply due to the fact that LIF expresses $C(\Delta, \ell)$ in terms of an integral, and the local operators correspond to singularities of $C(\Delta, \ell)$. In other words, the integral has no chance of converging near the values of $\Delta, \ell$ relevant to local operators, except perhaps for leading-twist operators (see [104] for steps in this direction). This necessarily makes any conclusions about anomalous dimensions of local operators reliant on additional assumptions. These are easy to justify in some perturbative expansions, but in non-perturbative setting do not appear to have been solidly understood.

Light-ray operators. Ref. [55] generalized LIF to external operators with spin and uncovered an interesting relation to Knapp-Stein intertwining operators, especially to what they called the light transform. They interpreted the analyticity of LIF in $\ell$ in terms of families of non-local non-integer-spin operators, the light-ray operators. These operators are defined for generic complex $\ell$ and reduce to light-transforms (null integrals) of local 
operators for integer spins. More recently, light-ray operators have been used to understand an OPE for event-shape observables such as energy-energy correlators in CFT [121-123] (see also $[124,125])$. The light-ray operators correspond to poles in $\Delta$ of $C(\Delta, \ell)$, and the issues with convergence of LIF described above prevent a simple rigorous proof of their nonperturbative existence. (E.g., for generic $\ell, C(\Delta, \ell)$ could have cuts or a natural boundary of analyticity in $\Delta$.) It would be interesting to find such a proof. In addition to clarifying the nature of light-ray operators, it would probably also have a bearing on the light-cone bootstrap results discussed above.

Conformal Regge theory provides a way to understand Minkowski correlators in Regge limit, and was developed in refs. [126-129]. Regge limit in CFT is a limit of a 4-point function in Lorentzian signature in which $\mathcal{O}_{2}$ approaches the "image of $\mathcal{O}_{3}$ in the next Poincaré patch," in 4-point function with the ordering

$$
\left\langle\mathcal{O}_{4} \mathcal{O}_{3} \mathcal{O}_{2} \mathcal{O}_{1}\right\rangle
$$

The operators $\mathcal{O}_{1}$ and $\mathcal{O}_{4}$ are kept spacelike separated, with $\mathcal{O}_{1}$ in past of $\mathcal{O}_{2}$ and $\mathcal{O}_{4}$ in the future of $\mathcal{O}_{3} .{ }^{90}$ The image of $\mathcal{O}_{3}$ in the next Poincare patch is the first point on Minkowski cylinder where all future-directed null geodesics from $\mathcal{O}_{3}$ meet. A lot of interest in Regge limit comes from its interpretation as bulk high-energy scattering through AdS/CFT. Kinematically, this limit is somewhat similar to the $\mathcal{O}_{2} \rightarrow \mathcal{O}_{3}$ limit because $\mathcal{O}_{3}$ and its image in the next Poincaré patch transform in the same way under conformal group. For example, the cross-ratios $z_{t}, \bar{z}_{t} \rightarrow 0$ in Regge limit. (Here by $z_{t}, \bar{z}_{t}$ we mean the cross-ratios for t-channel $\mathcal{O}_{2} \times \mathcal{O}_{3}$.) However, they do so after $\bar{z}_{t}$ crosses the cut $[1, \infty)$, and so in terms of $\rho_{t}, \bar{\rho}_{t}$ we have $\rho_{t} \rightarrow 0$ and $\bar{\rho}_{t} \rightarrow \infty$. Therefore, the $\mathcal{O}_{2} \times \mathcal{O}_{3}$ OPE is divergent. Conformal Regge theory gives a way to resum the $\mathcal{O}_{2} \times \mathcal{O}_{3}$ OPE in a way that exhibits a dominant contribution from a "Reggeon" exchange, which is an example of a light-ray operator. Justification for this resummation, which involves analytic continuation of OPE data in spin, comes from LIF (which historically was understood after Conformal Regge theory was established). In the context of our paper, it would be interesting to understand whether such resummations can be made rigorous enough (in axiomatic sense) and used to prove that Minkowski correlators are functions in regions where so far only temperedness has been proven. ${ }^{91}$ For this it might not be necessary to understand the Reggeon or more general light-ray operators, since the resummation procedure can be stopped at a point where the correlator is expressed as an integral of $C(\Delta, \ell)$ over a region where LIF converges. See [92] for progress on these questions.

Works of Gillioz, Luty et al. Papers by this group of authors are characterized by the systematic use of momentum space in Lorentzian CFT. So, refs. [60, 80] computed Lorentzian momentum space 3 -point functions (3 scalars and scalar-scalar-spin $\ell$ ) by solving

\footnotetext{
${ }^{90}$ In a symmetric version of the limit, which is related to the one described here by a conformal transformation, the operators $\mathcal{O}_{1}$ and $\mathcal{O}_{4}$ approach each other's images in the same way as $\mathcal{O}_{2}$ and $\mathcal{O}_{3}$ do.

${ }^{91}$ In the classic Regge limit there is a channel in which the OPE converges regularly, but it is possible that some causal orderings can be relaxed while keeping the resummation procedure valid.
} 
the conformal Ward identities. In momentum space, it's also possible to form conformal blocks by gluing 3-point functions [80]. See also notes 40, 67 .

Ref. [130] carried out this program quite explicitly in 2d CFT, with an eye towards eventual conformal bootstrap applications. They stressed that the momentum conformal block expansion generally converges only in the sense of distributions - one of the first mentions of distributional convergence in the modern CFT literature. For some momenta configurations, they argued that the momentum conformal blocks can be pointwise bounded by the position conformal blocks with an appropriately chosen real $z \in(0,1)$. For such configurations the momentum expansion converges in the ordinary sense of functions. The same work also proposes a bootstrap equation in the momentum space, obtained by transforming the local commutativity constraint multiplied by a test function selecting configurations with a spacelike pair of points (however, examples of test functions chosen in [130] may be too singular).

Refs. [131-133] studied the Fourier transform of the time-ordered Minkowski 4-point function in relation to various interesting physics questions. Note that, as mentioned in the conclusions, time-ordered Minkowski CFT 4-point functions have not yet been rigorously defined as a distributions. The Fourier transform depends on 4 momenta $p_{i}$, and to reduce functional complexity it is interesting to take some or all of these momenta lightlike, $p_{i}^{2} \rightarrow 0$.

So, ref. [131] considered the Fourier transform of the connected time-ordered 4-point function $\left\langle\mathcal{T}\left\{\mathcal{O}_{1} \mathcal{O}_{2} \mathcal{O}_{3} \mathcal{O}_{4}\right\}\right\rangle_{c}$. Here they worked with operators of scaling dimension $\Delta_{i}>$ $d / 2$ for which the Fourier transform is expected to have a finite limit as $p_{i}^{2} \rightarrow 0 .{ }^{92}$ Ref. [131] proposed a Lorentzian CFT analogue of the optical theorem:

$$
\operatorname{Im} \mathcal{M}_{1234}(s, t)=\sum_{\mathcal{O} \neq 1} f_{12 \mathcal{O}} f_{43}^{*} \mathcal{O}_{\mathcal{O}}(q)\left\langle\overline{\mathcal{T}}\left\{\widehat{\mathcal{O}}_{1}\left(p_{1}\right) \widehat{\mathcal{O}}_{2}\left(p_{2}\right)\right\} \mathcal{O}^{\dagger}(0)\right\rangle\left\langle\mathcal{O}(0) \mathcal{T}\left\{\widehat{\mathcal{O}}_{3}\left(p_{3}\right) \widehat{\mathcal{O}}_{4}\left(p_{4}\right)\right\}\right\rangle
$$

where $\mathcal{M}_{1234}$ is proportional to the Fourier transform of $\left\langle\mathcal{T}\left\{\mathcal{O}_{1} \mathcal{O}_{2} \mathcal{O}_{3} \mathcal{O}_{4}\right\}\right\rangle_{c}, f_{i j k}$ is the same as in $(2.33), \mathcal{N}_{\mathcal{O}}(q)$ is some normalization factor at $q=p_{1}+p_{2}\left(=-p_{3}-p_{4}\right)$, and $\widehat{\mathcal{O}}$ denotes the Fourier transform of $\mathcal{O}$. Eq. (A.2) is supposed to hold in the following kinematic region in the momentum space:

$$
p_{i}^{2}=0, \quad s=\left(p_{1}+p_{2}\right)^{2}>0, \quad t=\left(p_{1}+p_{3}\right)^{2} \leqslant 0,
$$

and was derived from a combinatorial operator identity

$$
\sum_{k=0}^{n}(-1)^{k} \sum_{\sigma \in S_{n}} \frac{1}{k !(n-k) !} \overline{\mathcal{T}}\left\{\mathcal{O}_{\sigma_{1}}\left(x_{\sigma_{1}}\right) \ldots \mathcal{O}_{\sigma_{k}}\left(x_{\sigma_{k}}\right)\right\} \mathcal{T}\left\{\mathcal{O}_{\sigma_{k+1}}\left(x_{\sigma_{k+1}}\right) \ldots \mathcal{O}_{\sigma_{n}}\left(x_{\sigma_{n}}\right)\right\}=0
$$

summing over all permutations, with $\mathcal{T}(\overline{\mathcal{T}})$ time ordering (anti-time ordering). Note that the use of this identity may be not fully safe in the distributional context, as it arises from a non-smooth partition of unity.

\footnotetext{
${ }^{92}$ We thank Marc Gillioz for explanations of his work and in particular of the distinction between the high dimension case discussed here and the low dimension case below.
} 
The CFT optical theorem (A.2) was used in ref. [131] to study the scale anomalies that appear in a specific class of CFT correlation functions. In fact, unlike Wightman functions which are conformally invariant distributions, time-ordered correlator distributions may, for certain scaling dimensions, contain pieces which violate scale invariance. Thus the scale anomaly describes the violation of dilatation Ward identities, and in position space it is an ultralocal term, located at coincident points. In Fourier space, scale anomaly translates into a nonzero imaginary part of $\mathcal{M}_{1234}(s, t)$ at $t=0$. Eq. (A.2) then computes the scale anomaly coefficient through a positive definite sum rule (in particular predicts that it is positive). These scale anomalies also appear in the Euclidean signature, and a similar sum rule for anomaly coefficients can also be found in ref. [131]. However, the Euclidean sum rule is not positive definite unlike the Lorentzian case.

Ref. [131] tested the above ideas for the scalar 4-point function of external dimensions $\Delta=3 d / 4$. Ref. [132] then studied the more interesting case of the stress tensor 4-point function $\left\langle\mathcal{T}\left\{T_{\mu_{1} \nu_{1}} T_{\mu_{2} \nu_{2}} T_{\mu_{3} \nu_{3}} T_{\mu_{4} \nu_{4}}\right\}\right\rangle$ whose scale anomaly is proportional to the stress-tensor 2-point function coefficient $c_{T}$. Assuming that the $t \rightarrow 0$ limit is finite, the CFT optical theorem expresses $c_{T}$ as a sum of positive contributions of all operators in the $T \times T$ OPE apart from the identity (the stress tensor contribution is known, proportional to $c_{T}$, and can be moved to the l.h.s.). The contributions from the scalars and the spin-2 operators are computed explicitly in [132].

The more recent ref. [133] studied instead the Fourier transform of the time-ordered 4-point function (or Euclidean 4-point function) in the opposite case of the low external dimensions $\Delta_{\phi}<d / 2$. Unlike in [131, 132], in this low dimension case the Fourier transform is singular as $p_{i}^{2} \rightarrow 0$, and one obtains a finite quantity multiplying it by $\left(p_{i}^{2}\right)^{d / 2-\Delta_{\phi}}$ before taking the limit, a CFT analogue of LSZ reduction. Doing so, they defined a "CFT scattering amplitude" $A(s, t, u)\left(p_{i}^{2} \rightarrow 0\right.$ for all $\left.i\right)$ and a closely related "form factor" $F(s, t, u)$ where $p_{i}^{2} \rightarrow 0$ only for $i=1,2,3$. Because the limit $p_{i}^{2} \rightarrow 0$ has to be taken one momentum at a time, crossing symmetry is not obvious. Ref. [133] also gave an alternative derivation, starting from the Mellin representation of the CFT 4-point function, where crossing symmetry of $F(s, t, u)$ and $A(s, t, u)$ follows from the crossing symmetry of the Mellin amplitude. In the future, crossing symmetric quantities $A(s, t, u)$ and $F(s, t, u)$ may turn out useful in a bootstrap analysis. It should be stressed that the results of [133] in no way contradict the usual lore that there are no S-matrices in interacting CFTs. In spite of the name adopted in [133], the existence of the quantity $A(s, t, u)$ does not imply that we can set up a wave-packet scattering experiment in a CFT. Wave-packets would quickly diffuse before reaching the interaction region, the singularity of $\left(p_{i}^{2}\right)^{\Delta_{\phi}-d / 2}$ being a cut rather than a pole.

\section{A.1 Review of Hartman et al. [4]}

Relating different orderings via analytic continuations. Here we will comment on some of the results of [4] in more details. The first part of this paper considers the Lorentzian CFT 4-point functions with operators $\mathcal{O}_{1}, \mathcal{O}_{3}, \mathcal{O}_{4}$ fixed at zero time and the spatial positions $0, \widehat{e}_{1}$ and $\infty$, while the operator $\mathcal{O}_{2}$ is inserted at Minkowski position 
$t_{2} \widehat{e}_{0}+y_{2} \widehat{e}_{1}$. They consider four different operator orderings

$$
\left\langle\mathcal{O}_{2} \mathcal{O}_{1} \mathcal{O}_{3} \mathcal{O}_{4}\right\rangle,\left\langle\mathcal{O}_{1} \mathcal{O}_{2} \mathcal{O}_{3} \mathcal{O}_{4}\right\rangle,\left\langle\mathcal{O}_{3} \mathcal{O}_{2} \mathcal{O}_{1} \mathcal{O}_{4}\right\rangle,\left\langle\mathcal{O}_{1} \mathcal{O}_{3} \mathcal{O}_{2} \mathcal{O}_{4}\right\rangle
$$

in the region of $0<y_{2}<1 / 2$ and $t_{2}$ positive. As $t_{2}$ is increased from zero, the operator $\mathcal{O}_{2}$, initially spacelike with respect to all other insertions, crosses the light cone first of $\mathcal{O}_{1}$ and then of $\mathcal{O}_{3}$. With $z, \bar{z}=y_{2} \pm t_{2}$, denoting $G(z, \bar{z})=\left\langle\mathcal{O}_{2} \mathcal{O}_{1} \mathcal{O}_{3} \mathcal{O}_{4}\right\rangle$, they give the following prescription to compute the correlators for the other orderings (ref. [4], eq. (3.22)):

$$
\begin{aligned}
\left\langle\mathcal{O}_{1} \mathcal{O}_{2} \mathcal{O}_{3} \mathcal{O}_{4}\right\rangle & =G(z, \bar{z})_{z \rightarrow e^{-2 \pi i} z}, \\
\left\langle\mathcal{O}_{3} \mathcal{O}_{2} \mathcal{O}_{1} \mathcal{O}_{4}\right\rangle & =G(z, \bar{z})_{(\bar{z}-1) \rightarrow e^{-2 \pi i}(\bar{z}-1)}, \\
\left\langle\mathcal{O}_{1} \mathcal{O}_{3} \mathcal{O}_{2} \mathcal{O}_{4}\right\rangle & =G(z, \bar{z})_{z \rightarrow e^{-2 \pi i} z,\left(\bar{z}-\bar{z}_{0}\right) \rightarrow e^{-2 \pi i}\left(\bar{z}-\bar{z}_{0}\right)} .
\end{aligned}
$$

Their justification of this prescription relied on some presumed analyticity properties of $G(z, \bar{z})$ which, to our knowledge, have never been shown in a general QFT context. Nevertheless we will see below that for CFTs eq. (A.5) turns out to be true (with $\bar{z}_{0}=1$ ).

The real parameter $\bar{z}_{0}$ was introduced in [4] as the position of the first $\bar{z}$ singularity of $G(z, \bar{z})_{z \rightarrow e^{-2 \pi i} z}$. Their goal was to show that $\bar{z}_{0} \geqslant 1$, which using (A.5) then implies local commutativity $\left\langle\mathcal{O}_{1}\left[\mathcal{O}_{2}, \mathcal{O}_{3}\right] \mathcal{O}_{4}\right\rangle=0$ for $\bar{z}<1$ i.e. when $\mathcal{O}_{2}$ is spacelike to $\mathcal{O}_{3}$. In our paper (section 6.9) we presented a different way to understand and derive local commutativity which is closer to the classic literature: as we have reviewed there, it is a robust consequence of the existence of the analytic continuation to the forward tube, which we constructed.

Let's see what it would take to justify (A.5). We define the function $G(z, \bar{z})$ by the $\mathcal{O}_{2} \times \mathcal{O}_{1}$ OPE expansions, which converges for $|\rho|,|\bar{\rho}|<1$, i.e. as long as $z, \bar{z}$ stay away from $z, \bar{z} \in(+1, \infty)$. The points $z=0$ and $\bar{z}=0$ are branch points singularities with cuts which we put along the negative real axis. Note that the contours in (A.5) are all within the analyticity domain of $G(z, \bar{z})$. So the prescription (A.5) is meaningful. We still have to see if it agrees with the rigorous definition which computes the Minkowski 4-point function by analytically continuing from the Euclidean region staying in the forward tube corresponding to the chosen operator ordering. We will see that it will indeed agree, but showing it for the last ordering will be subtle.

For definiteness we will focus on the region $t_{2}>1-y_{2}$ i.e. $\bar{z}>1$, where $\mathcal{O}_{2}$ crossed both light cones. The end point of the analytic continuation contour is always the same while the initial point depends on the operator ordering. E.g. for the ordering $\left\langle\mathcal{O}_{2} \mathcal{O}_{1} \mathcal{O}_{3} \mathcal{O}_{4}\right\rangle$ we have to pick initial Euclidean times $\varepsilon_{2}>\varepsilon_{1}>\varepsilon_{3}$, while for $\left\langle\mathcal{O}_{1} \mathcal{O}_{2} \mathcal{O}_{3} \mathcal{O}_{4}\right\rangle$ we have $\varepsilon_{1}>\varepsilon_{2}>\varepsilon_{3}$ etc. For any of these orderings, we denote

$$
z_{1}, \bar{z}_{1}= \pm i \varepsilon_{1}, \quad z_{2}, \bar{z}_{2}=y_{2} \pm i\left(\varepsilon_{2}+i t_{2}\right), \quad z_{3}, \bar{z}_{3}=1 \pm i \varepsilon_{3}
$$

and compute (in the limit $z_{4}, \bar{z}_{4}=\infty$ )

$$
z=\frac{z_{1}-z_{2}}{z_{1}-z_{3}}, \quad \bar{z}=\frac{\bar{z}_{1}-\bar{z}_{2}}{\bar{z}_{1}-\bar{z}_{3}} .
$$




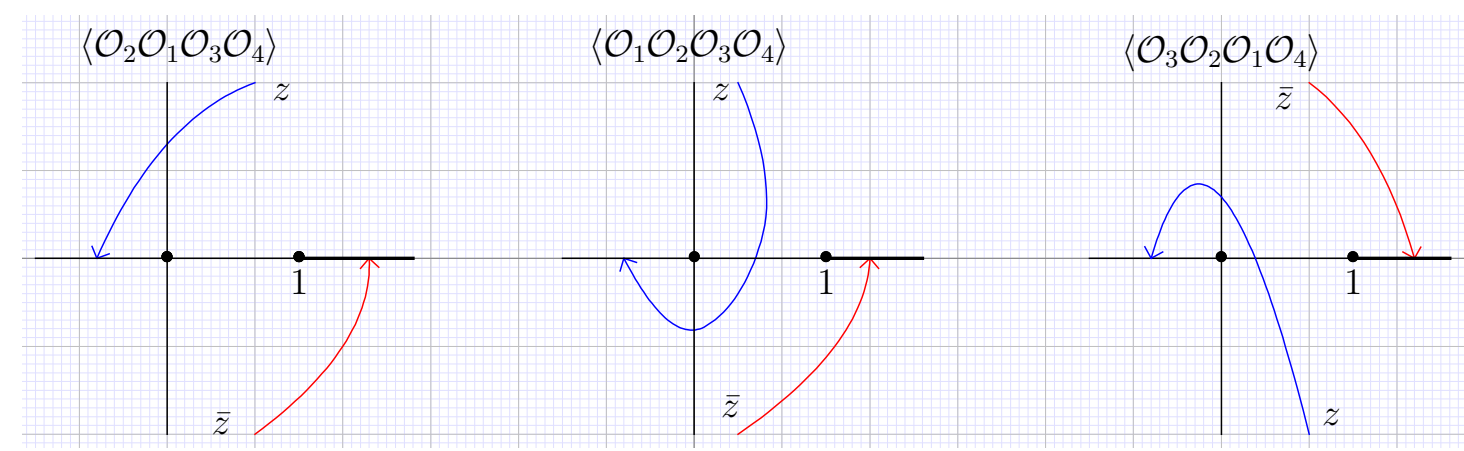

Figure 11. $z, \bar{z}$ curves for the analytic continuation from the Euclidean; the first 3 orderings.

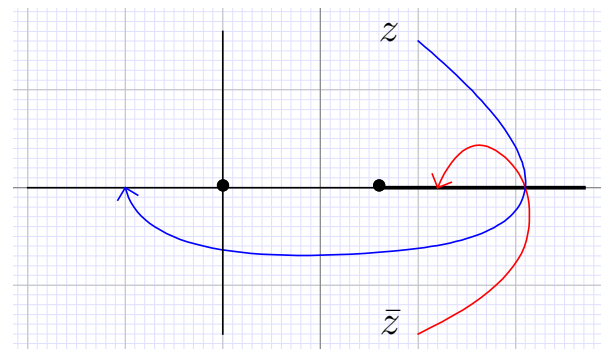

Figure 12. $z, \bar{z}$ curves for the analytic continuation from the Euclidean for the $\left\langle\mathcal{O}_{1} \mathcal{O}_{3} \mathcal{O}_{2} \mathcal{O}_{4}\right\rangle$ ordering.

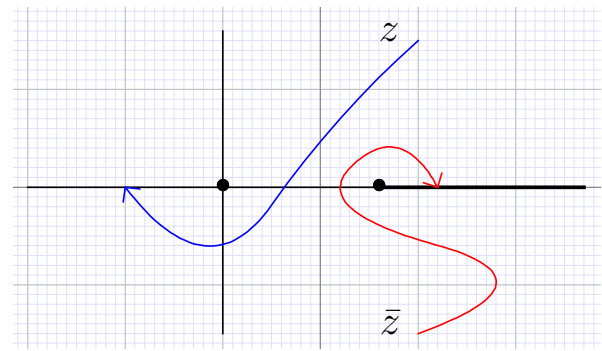

Figure 13. $z, \bar{z}$ curves for computing the $\left\langle\mathcal{O}_{1} \mathcal{O}_{3} \mathcal{O}_{2} \mathcal{O}_{4}\right\rangle$ ordering via (A.5).

We are interested in the curves which $z, \bar{z}$ trace as the Euclidean times are scaled to zero and the Minkowski time $t_{2}$ from 0 to its final value. For the first three orderings the resulting curves are shown in figure 11.

We see that in all these cases, the curves lie in the analyticity domain of $G(z, \bar{z})$ i.e. they don't cross $(1,+\infty)$. For the first two cases this was guaranteed by our results that $|\rho|,|\bar{\rho}|<1$ for the s-channel OPE expansion. For the third case it was not guaranteed but it also turns out to be true, by inspection. We also see that in all these 3 cases, the curves go around $z=0$ and $\bar{z}=1$ in agreement with (A.5). ${ }^{93}$

For the fourth ordering $\left\langle\mathcal{O}_{1} \mathcal{O}_{3} \mathcal{O}_{2} \mathcal{O}_{4}\right\rangle$ when we have to assign $\varepsilon_{1}>\varepsilon_{3}>\varepsilon_{2}$, the analytic continuation inside the forward tube gives the $z, \bar{z}$ curves shown in figure 12 , while prescription (A.5) would correspond to figure 13.

\footnotetext{
${ }^{93}$ To see this more clearly in the third case, deform the curves continuously moving the initial $z$ into the upper half plane and the initial $\bar{z}$ into the lower half plane.
} 


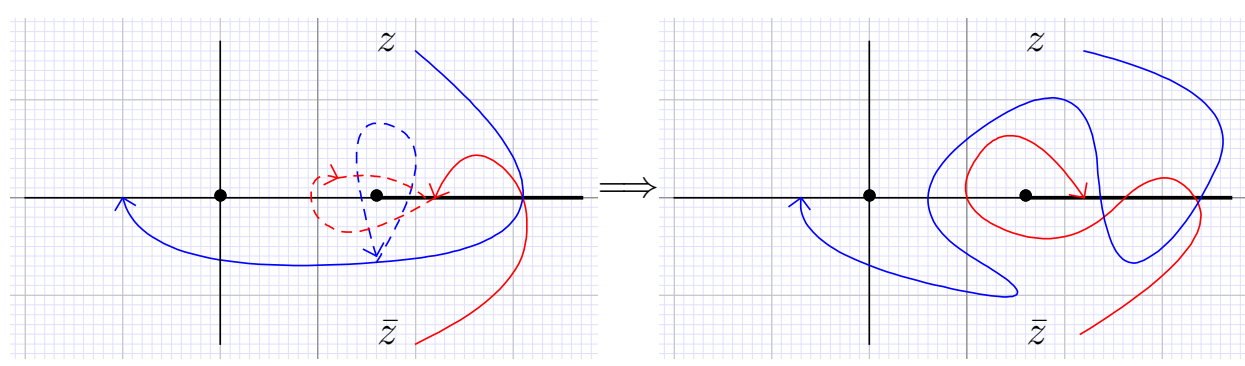

Figure 14. Deforming the $z, \bar{z}$ curves for the $\left\langle\mathcal{O}_{1} \mathcal{O}_{3} \mathcal{O}_{2} \mathcal{O}_{4}\right\rangle$ ordering.

The two figures are clearly not the same. Moreover the curves in the first figure cross $(+1, \infty)$ where the definition of $G(z, \bar{z})$ via the $\mathcal{O}_{1} \times \mathcal{O}_{2}$ channel OPE expansion stops converging. Can we show that the analytic continuation in figure 12 exists and that it agrees with the one in figure 13 ?

For this, let us bring in the $\mathcal{O}_{2} \times \mathcal{O}_{3}$ OPE expansion, which correspond to expanding in $z_{t}=1-z, \bar{z}_{t}=1-\bar{z}$ or in the corresponding $\rho_{t}, \bar{\rho}_{t}$. In the Euclidean region the two expansions agree. The $\mathcal{O}_{2} \times \mathcal{O}_{3}$ expansion converges away from $z_{t}, \bar{z}_{t} \in(+1, \infty)$ i.e. $z, \bar{z} \in(-\infty, 0)$. Thus the curves in both figures 12, 13 lie within the range of analyticity of the $\mathcal{O}_{2} \times \mathcal{O}_{3}$ expansion, so we can compare the analytic continuations. Since only integer spins occur in the expansion, the analytic continuation does not change under $\rho_{t} \rightarrow e^{2 \pi i} \rho_{t}, \bar{\rho}_{t} \rightarrow e^{-2 \pi i} \bar{\rho}_{t}$ (such arguments were systematically exploited in [37]). ${ }^{94}$ So let us add extra loops to the blue and the red curves in the opposite directions around 1 , see figure 14. Adding the loops and deforming the curves continuously (the first step is shown in figure 14) we can bring them to those in figure 13. This finishes the proof that the prescription (A.5) is correct also for the fourth ordering.

Positivity constraints. We wish to comment on another result of [4]: an argument for positivity of certain conformal block expansion coefficients. We present the argument exchanging the role of s an $\mathrm{t}$ channels w.r.t. [4]. Let $G(z, \bar{z})=1+\ldots$ be the holomorphic function defined by the s-channel OPE expansion (i.e. $(z \bar{z})^{\Delta_{1}+\Delta_{2}}$ times the $G(z, \bar{z})$ discussed above). We will define a certain analytic continuation of the function $G(z, \bar{z})$. Let us start with $\bar{z}$ and $z$ close to zero, $\bar{z}$ in the upper half plane and $z$ in the lower half plane. In this range all three channels s,t,u converge. We wish to analytically continue $G(z, \bar{z})$ by taking $z$ through $(1,+\infty)$ and bring it back close to zero, in the upper half plane (see figure 15), while we don't touch $\bar{z}$. This analytic continuation can be performed using the t-channel or $\mathrm{u}$-channel expansions, with the same result (but not the s-channel since it stops converging on $(1,+\infty))$. We denote the result of this analytic continuation by $\widehat{G}(z, \bar{z})$, with $\operatorname{Im} z, \operatorname{Im} \bar{z}>0$.

Although $\widehat{G}(z, \bar{z})$ is so defined with both $z, \bar{z}$ in the upper half plane, it has continuous limits when they both approach positive real axis, or both approach negative real axis, since in the first case the t-channel and in the second case the u-channel remains convergent. We will be interested in the situation when $\mathcal{O}_{1}=\mathcal{O}_{2}, \mathcal{O}_{3}=\mathcal{O}_{4}$. In this case all expansion

\footnotetext{
${ }^{94}$ Sometimes this property is called "Euclidean single-valuedness".
} 


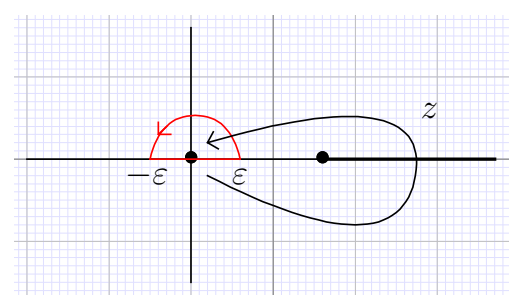

Figure 15. Definition of $\widehat{G}(z, \bar{z})$. Red: integration contour in eq. (A.11).

coefficients in the $\mathrm{t}$ and $\mathrm{u}$ channels are positive. This implies that the Euclidean correlator $G_{E}(z, \bar{z})$ will be positive for real $z, \bar{z}>0$ (using t-channel) and for real $z, \bar{z}<0$ (using u-channel). The difference between $G_{E}(z, \bar{z})$ and $\widehat{G}(z, \bar{z})$, for real $z, \bar{z}>0$ or $z, \bar{z}<0$ is that in the first case $z, \bar{z}$ approach the real axis from the opposite sides while in the second case from the same one. When we take $z$ through $(1,+\infty)$ cut, this only brings in some phases in the $\mathrm{t}$ and $\mathrm{u}$-channel expansion of $\widehat{G}(z, \bar{z})$ with respect to $G_{E}(z, \bar{z})$. This implies that we have a bound for real $z, \bar{z}>0$ or $z, \bar{z}<0$ :

$$
|\widehat{G}(z, \bar{z})| \leqslant G_{E}(z, \bar{z})
$$

In what follows $\widehat{G}(z, \bar{z})$ will be used as a holomorphic function with $z, \bar{z}$ in the upper half plane satisfying the bound (A.8) on its boundary, while $G_{E}(z, \bar{z})$ will be used only with real $z, \bar{z}$.

In particular, since $G_{E} \approx 1$ for $z, \bar{z}$ near zero up to small corrections, eq. (A.8) says that $\widehat{G}$ is bounded, for small real $z, \bar{z}>0$ or $z, \bar{z}<0$, by 1 up to small corrections. This argument can be generalized to show that $\widehat{G}(z, \eta z)$ for $\eta>0$ real and $z$ near zero in the upper half plane is bounded by a constant. ${ }^{95}$

We now pass to the non-rigorous part of the argument. Although the s-channel stops converging when crossing $(1,+\infty)$, ref. [4] proposed that, in the regime $|\bar{z}| \ll|z| \ll 1$, the behavior $\widehat{G}(z, \bar{z})$ can nevertheless be predicted from the s-channel expansion, by organizing it in $\bar{z}^{\tau / 2}$ where $\tau=\frac{1}{2}(\Delta-\ell)$ is the twist. The typical term is

$$
\bar{z}^{\tau / 2} k_{\frac{1}{2}(\Delta+\ell)}(z)
$$

where $k_{h}(x)={ }_{2} F_{1}(h, h, 2 h, x)$ is the collinear conformal block. This is the same expansion as used in the light-cone bootstrap, which has its own problems of rigor, but here it is proposed to use it after $z-1 \rightarrow e^{2 \pi i}(z-1)$. Under this continuation the collinear conformal block, which has a $\log (1-z)$ behavior near $z=1$, picks up an imaginary piece which, for $z$ small, behaves as $\sim i z^{1-\frac{1}{2}(\Delta+\ell)}$ (see [4], eq. (4.28)). Considering $\bar{z}=\eta z, \eta \ll 1,|z| \ll 1$, we then have, according to the proposal of ref. [4],

$$
\widehat{G}(z, \eta z) \approx 1-B(\Delta, \ell) p_{\Delta, \ell} \times i \frac{\eta^{\tau / 2}}{z^{\ell-1}},
$$

\footnotetext{
${ }^{95}$ Let $z=r e^{i \varphi}, r \ll 1$. We consider $0 \leqslant \varphi \leqslant \pi / 2$, when the argument uses the t-channel, the case $\pi / 2 \leqslant \varphi \leqslant \pi$ is analogous using the u-channel. The key point is that the $\rho$ variable in the t-channel $\rho_{t} \approx 1-\sqrt{r} e^{i \varphi / 2},\left|\rho_{t}\right| \approx 1-\sqrt{r} \cos (\varphi / 2)$. This allows to compare the function $\widehat{G}(z, \eta z)$ to $G_{E}\left(z^{\prime}, \eta z^{\prime}\right)$ with real $z^{\prime}=r \cos ^{2}(\varphi / 2)$, times a factor $\sim(z \bar{z})^{\Delta_{1}} /\left(z^{\prime} \bar{z}^{\prime}\right)^{\Delta_{1}}$ from the crossing kernel, which is bounded by a constant.
} 
where $\Delta, \ell$ are the dimension and spin of the leading twist operator (which may e.g. be the stress tensor), $p_{\Delta, \ell}$ its conformal block coefficient, and $B(\Delta, \ell) \geqslant 0$ some explicitly known constant. The spin $\ell$ is even since we are assuming $\mathcal{O}_{1}=\mathcal{O}_{2}$. Eq. (A.10) assumes that the limit $\eta \rightarrow 0$ is taken before $z \rightarrow 0 .{ }^{96}$

Now, let us consider the holomorphic function $f(z)=1-\widehat{G}(z, \eta z)$, and integrate $z^{\ell-2} f(z)$ along the contour shown in figure $15 .{ }^{97}$ We have

$$
\int_{\operatorname{arc}} z^{\ell-2} f(z) d z+\int_{-\varepsilon}^{\varepsilon} x^{\ell-2} f(x) d x=0 .
$$

Using (A.10) and that the integral over the arc of $1 / z$ is $\pi i$, we get in particular: ${ }^{98}$

$$
\begin{aligned}
\pi B(\Delta, \ell) p_{\Delta, \ell} & \approx \eta^{-\tau / 2} \int_{-\varepsilon}^{\varepsilon} x^{\ell-2} \operatorname{Re}[1-\widehat{G}(z, \eta z)] d x \\
& \approx \eta^{-\tau / 2} \int_{-\varepsilon}^{\varepsilon} x^{\ell-2} \operatorname{Re}\left[G_{E}(z, \eta z)-\widehat{G}(z, \eta z)\right] d x
\end{aligned}
$$

where in the final step we replaced 1 by $G_{E}(z, \eta z)$. Since $G_{E}(z, \eta z)$ has a rigorously convergent expansion for small $z$, it satisfies the bound:

$$
G_{E}(z, \bar{z})=1+O\left(\eta^{\tau / 2}\right)
$$

So the last replacement was legitimate if e.g. $\ell \geqslant 2$. By (A.8), the r.h.s. of (A.12) is a positive quantity. This equation then implies that $p_{\Delta, \ell}$ must be positive as well.

As already mentioned, the weak point of this argument is that the s-channel expansion stops converging when we cross $(1,+\infty)$. It is therefore not at all obvious that analytic continuations of the individual conformal block expansion terms have anything to do with the asymptotics of $\widehat{G}(z, \bar{z})$. Ref. [4] was of course aware of this, and provided some arguments, inspired by the light-cone bootstrap, why nevertheless the asymptotics from the leading twist terms can be trusted. We don't know how to make those arguments rigorous. It would be interesting to understand if asymptotics (A.10) can be justified using just Euclidean CFT axioms of section 2.3 or requires additional assumptions. The same question also looms over the proofs of ANEC [45] and ANEC commutativity [122] which involved similar "light-cone limit on the second sheet" considerations.

\section{B OS axioms for higher-point functions}

In this appendix we discuss the modifications necessary to derive from the Euclidean CFT axioms the OS axioms (positivity and cluster property) for $n$-point functions with $n>4$,

\footnotetext{
${ }^{96}$ In fact, in the opposite limit $z \rightarrow 0$ for fixed $\eta$, eq. (A.10) would violate the discussed above rigorous bound that $\widehat{G}(z, \eta z)$ is bounded by a constant. There is no paradox because that's not the limit we are supposed to be taking.

${ }^{97}$ We can also take an intermediate step of adding a small semicircle of radius $\varepsilon^{\prime}$ around zero, but since $\widehat{G}$ is bounded for small $z$, the limit $\varepsilon^{\prime} \rightarrow 0$ is not problematic.

${ }^{98}$ Note that the quantity $\operatorname{Re}\left[G_{E}(z, \eta z)-\widehat{G}(z, \eta z)\right]$ appearing in (A.12) is essentially the double discontinuity considered in [56]. Similarly, eq. (A.12) can be formally obtained from the Lorentzian inversion formula of [56] by expanding the integrand in a light-cone limit. We thank Tom Hartman for pointing this out.
} 
compared to the $n \leqslant 4$ case considered in section 3.1. As we explain below, it appears that there is no simple proof of OS positivity for $n>4$ from the Euclidean CFT axioms of section 2.3. Since the reason for this is rather technical, let us first discuss the conceptual implications of this.

Ideally, one would like to have a set of Euclidean CFT axioms that would imply Wightman axioms (and therefore also OS axioms) and also be powerful enough to derive all the usual CFT lore such as OPEs, radial quantization, operator-state correspondence, crossing symmetry, etc. These statements, as we saw in the main text, make sense and can be non-trivial even when we restrict our attention to $n$-point functions with bounded $n$.

In particular, we have found that the axioms we formulated in section 2.3 achieve the above goal for $n \leqslant 4$. Extending our results to $n>4$ using the same strategy would require a solution to two problems: first, we need to derive OS axioms (specifically, positivity and cluster property) for $n>4$, and, second, we need to prove that OS axioms together with the OPE imply Wightman axioms.

Conceptually, it seems plausible that OS axioms + OPE imply Wightman axioms for $n>4$ because we expect that for $n>4$ there is again an OPE channel which is convergent in the entire forward tube (i.e. the one given by taking the OPE in the same order as the operators appear in the Wightman ordering). This question clearly merits further study but is beyond the scope of this paper.

However, it is less clear to us how to even attempt a derivation of OS positivity for $n>4$ from Euclidean CFT axioms of section 2.3. Let us first explain why this is the case, and then we will discuss the possible modifications to these CFT axioms.

Suppose we want to prove the positivity

$$
\langle\Psi \mid \Psi\rangle \geqslant 0
$$

where $\Psi$ is a state created by a product of three local operators, $|\Psi\rangle=$ $\left|\varphi_{1}\left(x_{1}\right) \varphi_{2}\left(x_{2}\right) \varphi_{3}\left(x_{3}\right)\right\rangle$.

To prove this positivity the natural idea would be to use the OPE expansion repeatedly for the two copies of $\Psi$ and then use the positivity of the 2-point function. However, for this we need our OPE approximation for $\langle\Psi|$ to be conjugate to our approximation for $|\Psi\rangle$. This is non-trivial to achieve because we have to perform the OPEs one at a time. For example, we can first construct an approximation of $|\Psi\rangle$ in terms of a state $\left|\Psi^{\prime}\right\rangle$, created by single operator insertions, such that

$$
\left|\langle\Psi \mid \Psi\rangle-\left\langle\Psi \mid \Psi^{\prime}\right\rangle\right|<\varepsilon
$$

Similarly, we can construct a state $\left\langle\Psi^{\prime \prime}\right|$ such that

$$
\left|\left\langle\Psi^{\prime \prime} \mid \Psi^{\prime}\right\rangle-\left\langle\Psi \mid \Psi^{\prime}\right\rangle\right|<\varepsilon
$$

and thus

$$
\left|\left\langle\Psi^{\prime \prime} \mid \Psi^{\prime}\right\rangle-\langle\Psi \mid \Psi\rangle\right|<2 \varepsilon .
$$


These approximations are possible by the repeated use of the OPE (3.2). Note, however, that since the OPE axiom is formulated for correlation functions, the number of terms we have to include in the OPE for a given $\varepsilon$ depends on the correlation function in which the OPE is performed. It then follows that the state $\left\langle\Psi^{\prime \prime}\right|$ depends on $\left|\Psi^{\prime}\right\rangle$ (because in order to construct it we use the OPE in the correlation function $\left.\left\langle\Psi \mid \Psi^{\prime}\right\rangle\right)$ and is in general different from it. It is therefore not obvious that $\left\langle\Psi^{\prime \prime} \mid \Psi^{\prime}\right\rangle \geqslant 0$, which is what we would like to use in order to prove $\langle\Psi \mid \Psi\rangle \geqslant 0$ with the help of the above inequalities.

In the case when $n=4$ and $|\Psi\rangle$ is created by 2 operators we were able to solve this difficulty. This was because in this case the only difference between $\left|\Psi^{\prime}\right\rangle$ and $\left\langle\Psi^{\prime \prime}\right|$ can be in the number of OPE terms included in the approximation, and we were able to use an orthogonality property of the 2-point function to show $\left\langle\Psi^{\prime \prime} \mid \Psi^{\prime}\right\rangle=\left\langle\Psi^{\prime} \mid \Psi^{\prime}\right\rangle$ by arguing that we can assume that $\left\langle\Psi^{\prime \prime}\right|$ contains more terms than $\left|\Psi^{\prime}\right\rangle$ and that those terms which are in $\left\langle\Psi^{\prime \prime}\right|$ but not in $\left|\Psi^{\prime}\right\rangle$ do not contribute to the product $\left\langle\Psi^{\prime \prime} \mid \Psi^{\prime}\right\rangle$.

This argument does not work in the case at hand, $|\Psi\rangle=\left|\varphi_{1}\left(x_{1}\right) \varphi_{2}\left(x_{2}\right) \varphi_{3}\left(x_{3}\right)\right\rangle$. The reason for this is that in order to construct $\left|\Psi^{\prime}\right\rangle$ or $\left\langle\Psi^{\prime \prime}\right|$ we need to perform two OPE's in each case. For example, the first one can be $\varphi_{1} \times \varphi_{2}=\sum_{k} \mathcal{O}_{k}$ and the second one can be $\varphi_{3} \times \mathcal{O}_{k}$. Both OPE's have to be truncated at some point, and while the truncation of the second OPE affects only the set of terms that are present in $\left|\Psi^{\prime}\right\rangle$ or $\left\langle\Psi^{\prime \prime}\right|$, where we truncate the first $\varphi_{1} \times \varphi_{2}$ OPE affects the coefficients of these terms. Since now $\left|\Psi^{\prime}\right\rangle$ and $\left\langle\Psi^{\prime \prime}\right|$ contain terms with differing coefficients, we cannot use orthogonality to argue $\left\langle\Psi^{\prime \prime} \mid \Psi^{\prime}\right\rangle=\left\langle\Psi^{\prime} \mid \Psi^{\prime}\right\rangle$ anymore. There is no way to ensure that $\varphi_{1} \times \varphi_{2}$ OPEs are truncated in the same way in the construction of both states because the truncation in $\left\langle\Psi^{\prime \prime}\right|$ depends, through our OPE axiom, on $\left|\Psi^{\prime}\right\rangle$, and thus might happen to be always at a higher order than the truncation used for $\left|\Psi^{\prime}\right\rangle$.

This all is to say that due to a rather technical reason it appears that there is no simple proof of OS positivity of higher-point functions from the Euclidean CFT axioms as stated in section 2.3. Importantly, this doesn't mean that there is no proof at all. Indeed, the Euclidean CFT axioms are sufficient to derive the standard crossing-symmetry equations for 4-point functions. It could happen that in all solutions to these crossing-symmetry equations the OPE coefficients have such asymptotics that a stronger form of the OPE axiom holds and allows us to prove the OS positivity for $n>4$. However, it is not clear how to implement this line of reasoning in practice.

It is therefore interesting to look for a stronger version of Euclidean CFT axioms. We discuss below some simple modifications of the OPE axiom which avoid the above problem and allow to prove OS positivity for higher-point functions.

Morally, we want some kind of statement of uniformity for the convergence rate of the OPE: it should make $\left|\Psi^{\prime \prime}\right\rangle$ above independent of the truncation made in $\left|\Psi^{\prime}\right\rangle$, as long as this truncation is done at a sufficiently high order. This would allow us to make both truncations at a high order and ensure $\left\langle\Psi^{\prime \prime} \mid \Psi^{\prime}\right\rangle=\left\langle\Psi^{\prime} \mid \Psi^{\prime}\right\rangle \geq 0$.

One option is to assume a stronger form of the OPE, which allows us to perform two OPE's simultaneously Specifically, we can assume that the double sum

$$
\left\langle\mathcal{O}_{1} \mathcal{O}_{2} \mathcal{O}_{3} \mathcal{O}_{4} \ldots\right\rangle=\sum_{k, l}\left\langle\mathcal{O}_{k} \mathcal{O}_{l} \ldots\right\rangle
$$


is convergent, where we wrote the two OPEs schematically as $\mathcal{O}_{1} \mathcal{O}_{2}=\sum_{k} \mathcal{O}_{k}$ and $\mathcal{O}_{3} \mathcal{O}_{4}=$ $\sum_{l} \mathcal{O}_{l}$. Convergence of the double sum means that

$$
\left|\left\langle\mathcal{O}_{1} \mathcal{O}_{2} \mathcal{O}_{3} \mathcal{O}_{4} \ldots\right\rangle-\sum_{k, l}\left\langle\mathcal{O}_{k} \mathcal{O}_{l} \ldots\right\rangle\right|<\varepsilon,
$$

when the sums are truncated in a way that includes some $\varepsilon$-dependent finite set of terms, but is otherwise arbitrary. In particular, both sums can be truncated in the same way, and this solves the problem that we encountered above. A disadvantage of this approach is that it is unclear how to derive this axiom from OS axioms and the usual single OPE axiom (however, a heuristic argument based on cutting the Euclidean path integral can be made). This is somewhat subtle and is related to the question of whether the path integral over a spherical layer $\left(r_{1}<r<r_{2}\right)$ with operator insertions in the interior represents a bounded operator. We can't say with confidence whether or not this is the case.

Another option is to assume resumed repeated OPE, i.e. that the following sum converges, schematically,

$$
\left\langle\mathcal{O}_{1} \ldots \mathcal{O}_{m} \mathcal{O}_{m+1} \ldots \mathcal{O}_{n}\right\rangle=\sum_{k} c_{k}\left\langle\mathcal{O}_{k} \mathcal{O}_{m+1} \ldots \mathcal{O}_{n}\right\rangle
$$

where the coefficients $c_{k}$ are chosen so that

$$
\left\langle\mathcal{O}_{1} \ldots \mathcal{O}_{m} \mathcal{O}_{k}^{\theta}\right\rangle=c_{k}\left\langle\mathcal{O}_{k} \mathcal{O}_{k}^{\theta}\right\rangle
$$

assuming $\left\langle\mathcal{O}_{k} \mathcal{O}_{l}^{\theta}\right\rangle \propto \delta_{k l}$. This version of the axiom is essentially the statement that oneoperator states form a basis of the CFT Hilbert space, formulated without explicitly introducing the Hilbert space. In other words, above we are approximating the state $\left\langle\mathcal{O}_{1} \ldots \mathcal{O}_{m}\right|$ in terms of an orthonormal basis of states $\left\langle\mathcal{O}_{k}\right|$, and the coefficients are computed by inner products. This form of the axiom is easy to derive from OS + convergent OPE, and also easily allows us to solve our problem by using the same strategy as in the case $n=4$. However, it does appear to be an overly strong assumption, making our axioms not very different from assuming OS + convergent OPE outright.

Finally, an interesting prospect might be, instead of formulating an entirely new set of axioms, to add an asymptotic OPE axiom (and conformal invariance) to OS axioms. It is likely that using logic very similar to that of Mack [41], which we reviewed in section 8.3, one can prove that (OS axioms) + (asymptotic OPE) + (conformal invariance) imply convergent OPE.

\section{Details on Vladimirov's theorem}

\section{C.1 Limit in the sense of distributions}

Let us start with a reminder of what the limit in the sense of tempered distributions means. Let $f(u), u=\left(t_{k}, \mathbf{x}_{k}\right) \equiv\left(t_{1}, \mathbf{x}_{1}, \ldots, t_{n}, \mathbf{x}_{n}\right) \in \mathbb{R}^{n d}$, be a Schwartz test function, i.e. an infinitely differentiable function decreasing at infinity faster than any power together with all its derivatives. This can be also stated as finiteness of all Schwartz norms:

$$
|f|_{N}=\sup _{u \in \mathbb{R}^{n d},|\alpha| \leqslant N}\left(1+u^{2}\right)^{N / 2}\left|\partial_{u}^{\alpha} f\right|<\infty \quad \forall N \geqslant 0 .
$$


That the limit (4.8) exists in the sense of distributions means two requirements. First, that the r.h.s. of (4.8) has a finite limit integrated against any $f$ as above:

$$
\left(G_{n}^{M}, f\right):=\lim _{\epsilon_{k} \rightarrow 0} \int d t d \mathbf{x} G_{n}\left(\epsilon_{k}+i t_{k}, \mathbf{x}_{k}\right) f\left(t_{k}, \mathbf{x}_{k}\right) \quad \text { exists for any Schwartz } f .
$$

The $G_{n}^{M}$ defined by this equation is a linear functional on the Schwartz space. The second requirement is that this functional should be continuous (and thus is itself a tempered distribution). Continuity means that it should be bounded by one of the norms (C.1) with a sufficiently large $N$, i.e.:

$$
\left|\left(G_{n}^{M}, f\right)\right| \leqslant C|f|_{N_{*}}
$$

with $f$-independent $C$ and $N_{*}$.

Note that by eq. (C.3), $G_{n}^{M}$ can be extended from the Schwartz space to a larger space of test functions, which are required to be differentiable only $N_{*}$ times and have a finite $|f|_{N_{*}}$. Parameter $N_{*}$ thus characterizes regularity of the distribution $G_{n}^{M}$. The proof of theorem 4.1 will determine $N_{*}$ in terms of $A_{n}$ and $B_{n}$, see eq. (C.6).

\section{C.2 Proof of theorem 4.1}

Unfortunately, we do not know a reference where theorem 4.1 is stated and proved succinctly in the form we need. Such results are considered standard in the theory of several complex variables. For similar statements see [134], Chapter 5, and [5], theorem 2-10. For the convenience of the reader, we present here a proof based on these sources.

The usefulness of Vladimirov's theorems for establishing distributional properties of CFT correlators was recognized in our recent work [36]. There, we considered expansions of the CFT 4-point function $g(\rho, \bar{\rho})$ in terms of conformally invariant cross-ratios $\rho, \bar{\rho}$. It is well known that such expansions converge in the interior of the unit disk $|\rho|,|\bar{\rho}|<1$. Using Vladimirov's theorems, we showed in [36] that they also converge on the boundary of this disk, in the sense of distributions. In this paper we are interested in CFT correlators as functions of positions $x_{k}$, not of cross-ratios, but the basic principle is the same as in [36]: a powerlaw bound on an holomorphic function near a boundary implies temperedness of the limiting distribution.

By translation invariance it's enough to study the function $G_{n}$ expressed in terms of the differences $y_{k}=x_{k}-x_{k+1}$ which we denote by $\mathcal{G}(y), y=\left(y_{1}, \ldots, y_{n-1}\right)$. We also denote $y_{k}=\left(y_{k}^{0}, \mathbf{y}_{k}\right), y_{k}^{0}=\varepsilon_{k}+i s_{k}, \varepsilon_{k}>0, \mathbf{y}_{k} \in \mathbb{R}^{d-1}$.

Consider first the case when all $\varepsilon_{k}$ go to zero together along a fixed direction: $\varepsilon_{k}=r v_{k}$ where $r \rightarrow 0$ and $v=\left(v_{k}\right)$ is a vector with positive components. Later on we will show that the limit continues to exist if $\varepsilon_{k} \rightarrow 0$ independently (as well as the more general statement about the limit from inside the forward tube).

So, let us prove that $\mathcal{G}(y)$ has a limit as $r \rightarrow 0$ which is a tempered distribution in variables $s_{k}, \mathbf{y}_{k}$. As in (C.2), we fix a Schwartz test function $f$ and consider the integral (we will omit index $k$ on $\varepsilon, s, v, \mathbf{y}$ if no confusion may arise)

$$
h(r)=\int d s d \mathbf{y} \mathcal{G}(r v+i s, \mathbf{y}) f(s, \mathbf{y}) .
$$


Te problem is analogous to theorems used in [36], so we will be brief. As in [36], section 3.3 and appendix $\mathrm{C}$, using analyticity in $y^{0}$, integration by parts, and the powerlaw bound one can show that derivatives of $h$ in $r$ satisfy the bound:

$$
\left|\partial_{r}^{j} h(r)\right| \leqslant \frac{C}{r^{A_{n}}}|f|_{N}
$$

where $|f|_{N}$ is a Schwartz norm (C.1) of a sufficiently large order $N$ depending on $j$ and $B_{n}$. The constant $A_{n}$ is the same as in (4.9), in particular the same $A_{n}$ works for all $j$. In what follows we only need this equation for finitely many $j$ (up to $\left[A_{n}\right]+1$ ). Using the Newton-Leibniz formula in the $r$ direction several times, one then proves that the same bound as (C.5) holds in fact without $1 / r^{A_{n}}$ singularity in the r.h.s. It then follows that, first of all, $\lim _{r \rightarrow 0} h(r)$ exists, and second, it is a continuous linear functional of $f$, that is, a distribution. The limit holds uniformly when the components $v_{k}$ vary on any fixed compact interval contained in $(0,+\infty)$. Its $v$-independence is shown exactly as in [36], eq. (C.7). Let us denote the limiting distribution $\mathcal{G}(i s, \mathbf{y}) \equiv \mathcal{G}^{M}(s, \mathbf{y})$.

It is of some interest to know the precise regularity of the distribution $\mathcal{G}^{M}$ (i.e. how many derivatives the test function must have to be pairable with $\mathcal{G}^{M}$ ) and the rate of its growth at infinity. Following the above argument in detail, one can show the following bound which contains this information:

$$
\left|\left(\mathcal{G}^{M}, f\right)\right| \leqslant \text { Const } . \int d s d \mathbf{y}(1+|s|+|\mathbf{y}|)^{B_{n}} \max _{|\alpha| \leqslant\left[A_{n}\right]+1}\left|\partial_{s}^{\alpha} f(s, \mathbf{y})\right| .
$$

This in particular implies (C.3) with $N_{*}=\max \left(\left[A_{n}\right]+1, B_{n}+n d+1\right)$.

Parts 2,3 of theorem 4.1 are new compared to [36], since such questions do not arise in the cross-ratio space.

Lorentz invariance is easy to show, as follows. Rotation invariance of $G_{n}^{E}$ implies that $\mathcal{G}(y)$ satisfies for real $y$ the differential equations

$$
\left\{y^{a} \partial_{y^{b}}-y^{b} \partial_{y^{a}}\right\} \mathcal{G}(y)=0, \quad a, b \in\{0,1, \ldots, d-1\}
$$

(as usual $y=\left(y_{k}\right)$, summation in $k$ understood). By the uniqueness of analytic continuation, these equations continue to hold for complex $y_{0}$. That's the only place where we use real-analyticity in the spatial direction. ${ }^{99}$ By taking the limit $\varepsilon \rightarrow 0$ in (C.7), we recover precisely the differential equations expressing the Lorentz invariance of $\mathcal{G}^{M}$. Let us explain in more detail how the limit is taken and why it exists. Consider for definiteness $a=0$, $b=1$, other cases being similar. Eq. (C.7) then says $\left\{(\varepsilon+i t) \partial_{y^{1}}+i y^{1} \partial_{t}\right\} \mathcal{G}(\varepsilon+i t, \mathbf{y})=0$, in the sense of functions, and hence integrating by parts in the sense of distributions acting on test functions $\varphi(t, \mathbf{y})$ :

$$
\left(\mathcal{G}_{\varepsilon},\left\{(\varepsilon+i t) \partial_{y^{1}}+i y^{1} \partial_{t}\right\} \varphi\right)=0,
$$

where we denoted $\mathcal{G}_{\varepsilon}(t, \mathbf{y})=\mathcal{G}(\varepsilon+i t, \mathbf{y})$. Now we take the limit $\varepsilon \rightarrow 0$. We know that (a) $\mathcal{G}_{\varepsilon} \rightarrow \mathcal{G}^{M}$ in the sense of distributions, and also that (b) $\left|\left(\mathcal{G}_{\varepsilon}, \varphi\right)\right|$ is uniformly

\footnotetext{
${ }^{99}$ With some extra tricks, it's possible to replace it by the assumption of mere continuity in $\mathbf{y}$, as in [3], theorem 4.3. In the CFT applications we have in mind, real analyticity appears a more natural assumption.
} 
bounded as $\varepsilon \rightarrow 0$ by some Schwartz norm of $\varphi$. By (b) the term $\left(\mathcal{G}_{\varepsilon}, \varepsilon \varphi\right)$ in (C.8) drops out when $\varepsilon \rightarrow 0$, and by (a) the rest tends to $\left(\mathcal{G}^{M},\left\{i t \partial_{y^{1}}+i y^{1} \partial_{t}\right\} \varphi\right)$. So we conclude that $\left(\mathcal{G}^{M},\left\{i t \partial_{y^{1}}+i y^{1} \partial_{t}\right\} \varphi\right)=0$ which expresses invariance of $\mathcal{G}^{M}$ under the 01 Lorentz transformation.

Let us proceed to show the rest of Parts 2,3. It will be crucial that $\mathcal{G}$ can be written as a "Fourier-Laplace transform":

$$
\mathcal{G}(\varepsilon+i s, \mathbf{y})=\int \frac{d E d \mathbf{p}}{(2 \pi)^{d(n-1)}} g(E, \mathbf{p}) e^{-(\varepsilon+i s) E-i \mathbf{p y}},
$$

where $g(E, \mathbf{p}), E \in \mathbb{R}^{n-1}, \mathbf{p} \in\left(\mathbb{R}^{d-1}\right)^{n-1}$ is a tempered distribution, called "spectral function", supported at $E \geqslant 0$ (by which we mean all $E_{k} \geqslant 0$ ) [later this will be improved to $E \geqslant|\mathbf{p}|]$. We are omitting the indices, thus $\varepsilon E=\sum_{k} \varepsilon_{k} E_{k}$, etc. The equality in (C.9) is understood in the sense of distributions, with the r.h.s. being the inverse Fourier transform of the tempered distribution $g(E, \mathbf{p}) e^{-\varepsilon E}$. In other words, what this means is that

$$
\int d s d \mathbf{y} \mathcal{G}(\varepsilon+i s, \mathbf{y}) f(-s,-\mathbf{y})=\int \frac{d E d \mathbf{p}}{(2 \pi)^{d(n-1)}} g(E, \mathbf{p}) e^{-\varepsilon E} \widehat{f}(E, \mathbf{p}),
$$

for any Schwartz test function $f$, and $\widehat{f}$ its Fourier transform.

Let us show (C.9). Notice first that for every $\varepsilon>0$ we can write

$$
\mathcal{G}(\varepsilon+i s, \mathbf{y})=\int \frac{d E d \mathbf{p}}{(2 \pi)^{d(n-1)}} g_{\varepsilon}(E, \mathbf{p}) e^{-i s E-i \mathbf{p y}},
$$

where $g_{\varepsilon}$ is the Fourier transform of $\mathcal{G}(\varepsilon+i s, \mathbf{y})$ with respect to $s, \mathbf{y}$. This Fourier transform exists as a tempered distribution, since $\mathcal{G}(\varepsilon+i s, \mathbf{y})$ is itself a tempered distribution in $s, \mathbf{y}$ (being a real-analytic function, bounded by a power at infinity). In addition, $\mathcal{G}(\varepsilon+i s, \mathbf{y})$ is differentiable in $\varepsilon$ and $s$ and satisfies the Cauchy-Riemann equations. From here it's easy to show that $g_{\varepsilon}$ as a distribution is differentiable in $\varepsilon$ and satisfies the differential equations:

$$
\frac{\partial g_{\varepsilon}}{\partial \varepsilon_{k}}+E_{k} g_{\varepsilon}=0 \quad(k=1, \ldots, d-1) .
$$

From here we conclude that

$$
g(E, \mathbf{p}):=g_{\varepsilon}(E, \mathbf{p}) e^{\varepsilon E}
$$

is an $\varepsilon$-independent distribution. Substituting $g_{\varepsilon}(E, \mathbf{p})=g(E, \mathbf{p}) e^{-\varepsilon E}$ into (C.11), we obtain (C.9). Note that since $g$ and $g_{\varepsilon}$ are related by an exponential factor, we can so far only claim that $g$ is defined as a distribution on test functions of compact support. Let us show next that it is in fact tempered (i.e. extends to Schwartz test functions).

To this end, consider the inverse of (C.11):

$$
g_{\varepsilon}(E, \mathbf{p})=g(E, \mathbf{p}) e^{-\varepsilon E}=\int d s d \mathbf{y} \mathcal{G}(\varepsilon+i s, \mathbf{y}) e^{i s E+i \mathbf{p y}},
$$

and integrate it against a compactly supported test function $\varphi(E, \mathbf{p})$. We get (compare (C.10)):

$$
\int d E d \mathbf{p} g(E, \mathbf{p}) e^{-\varepsilon E} \varphi(E, \mathbf{p})=\int d s d \mathbf{y} \mathcal{G}(\varepsilon+i s, \mathbf{y}) \widehat{\varphi}(-s,-\mathbf{y}) .
$$


As $\varepsilon \rightarrow 0$, the l.h.s. tends to the pairing $(g, \varphi)$. Using Part 1 of the theorem, the r.h.s. tends in the same limit to $\int d s d \mathbf{y} \mathcal{G}^{M}(s, \mathbf{y}) \widehat{\varphi}(-s,-\mathbf{y})$ which exists in the sense of tempered distributions and so is bounded by some Schwartz-space norm $|\widehat{\varphi}|_{N}$. We get

$$
|(g, \varphi)| \leqslant \text { const } .|\hat{\varphi}|_{N} \leqslant \text { const } .|\varphi|_{N^{\prime}}
$$

where in the second inequality we used that the Fourier transform is continuous in the Schwartz space. This inequality, valid for any compactly supported $\varphi$, means that $g$ extends to a tempered distribution on the whole Schwartz space. The representation (C.9) is thus established.

Next let us show that $g$ is supported at $E \geqslant 0$. For this we will pass to the large $\varepsilon$ limit in (C.13). Supposing that $E_{k}<0$ for some $k$, the factor $e^{E \varepsilon}$ in (C.13) decreases exponentially as the corresponding $\varepsilon_{k} \rightarrow+\infty$. On the other hand $g_{\varepsilon}(E, \mathbf{p})$ is bounded in the same limit by a power of $\varepsilon$, because it's the Fourier transform of $\mathcal{G}(\varepsilon+i s, \mathbf{y})$ which satisfies a powerlaw bound. ${ }^{100}$ This implies that $g(E, \mathbf{p})=0$ unless $E \geqslant 0 .{ }^{101}$

Consider then the following lemma, proven analogously to, and easier than, lemma C.2 below.

Lemma C.1. Let $g(E, \mathbf{p})$ be a tempered distribution supported at $E \geqslant 0$, and consider the distribution $g(E, \mathbf{p}) e^{-\varepsilon E}(\varepsilon>0)$. This distribution, being initially defined by this formula on compactly supported test functions, extends to a tempered distribution, and moreover $g(E, \mathbf{p}) e^{-\varepsilon E} \rightarrow g(E, \mathbf{p})$ as $\epsilon \rightarrow 0$, in the sense of tempered distributions.

Let us now take the $\varepsilon \rightarrow 0$ limit on both sides of (C.9) (or, which is the same, (C.10)). The 1.h.s. has a limit by Part 1, while the r.h.s. has a limit by lemma C.1. We obtain that $\mathcal{G}^{M}(s, \mathbf{y})$ and $g(E, \mathbf{p})$ are related by the Fourier transform:

$$
\mathcal{G}^{M}(s, \mathbf{y})=\int \frac{d E d \mathbf{p}}{(2 \pi)^{d(n-1)}} g(E, \mathbf{p}) e^{-i s E-i \mathbf{p y}} .
$$

We can now complete the proof of Part 2, namely to show the spectral condition. Above we proved that $\mathcal{G}^{M}(s, \mathbf{y})$ is Lorentz invariant. Since $g(E, \mathbf{p})$ is its Fourier transform, it is also Lorentz invariant, and in particular its support must be a Lorentz-invariant set. We also know that $\operatorname{supp} g \subset\{E \geqslant 0\}$. These two facts together imply that $\operatorname{supp} g$ must be contained in the product of the forward null cones, i.e. $g(E, \mathbf{p})=0$ unless each $E_{k} \geqslant\left|\mathbf{p}_{k}\right|$, which is the spectral condition.

Part 3 follows by standard Wightman theory arguments. Namely, let us write $\left(i y_{k}^{0}, \mathbf{y}_{k}\right)=\xi_{k}+i \eta_{k}$ where $\xi_{k}, \eta_{k} \in \mathbb{R}^{1, d-1}$ and $\eta_{k}=\left(\operatorname{Re} y_{k}^{0}, \operatorname{Im} \mathbf{y}_{k}\right) \succ 0$. The extension to the forward tube is given by the equation (with $p=(E, \mathbf{p})$ )

$$
\int d p g(p) e^{i(p, \xi)} e^{(p, \eta)}
$$

which reduces to (C.9) for real $\mathbf{y}_{k}$. It is holomorphic by Part (c) of the following lemma, while Parts (a,b) imply that this extension has the same limit as (C.9).

\footnotetext{
${ }^{100}$ This is the only place where we use the powerlaw bound on $\mathcal{G}(\varepsilon+i s, \mathbf{y})$ for large rather than small $\varepsilon$.

${ }^{101}$ If unhappy with this intuitive reasoning, the argument may be made more rigorous in its integrated version: show that $g$ vanishes on test functions supported in the complement of $E \geqslant 0$.
} 
Lemma C.2. Let $g(p)$ be a tempered supported at $p \succeq 0$ (closed forward light cone). Consider the distribution $g_{\eta}(p)=g(p) e^{-(p, \eta)}$, initially defined by this formula on compactly supported test functions. Then

(a) $g_{\eta}$ for $\eta \succ 0$ extends to a tempered distribution;

(b) $g_{\eta} \rightarrow g$ as $\eta \rightarrow 0$ from inside the forward light cone $\eta \succ 0$, in the sense of tempered distributions;

(c) The Fourier transform $\widehat{g_{\eta}}(\xi)$ of the distribution $g_{\eta}(p)$ is a holomorphic function of $\xi+i \eta$ for $\eta \succ 0$.

Proof. Let $\omega(p)$ be a $C^{\infty}$ function which is identically 1 on the forward light cone $\overline{V_{+}}$, and zero as soon as $\operatorname{dist}\left(p, \overline{V_{+}}\right) \geqslant 1$ where dist is the Euclidean distance. We can choose this function so that all its derivatives are uniformly bounded by a constant depending only on the derivative order: $\left|\omega^{(\alpha)}(p)\right| \leqslant C_{\alpha}$ for any $p$.

Consider the family of $C^{\infty}$ functions parametrized by $\xi, \eta \in \mathbb{R}^{1, d-1}$ :

$$
\Omega_{\xi, \eta}(p)=e^{i(p, \xi)} e^{(p, \eta)} \omega(p)
$$

It is not hard to check that $\Omega_{\xi, \eta}$ is a Schwartz function for $\eta \succ 0$ and any $\xi$.

Let us define $g_{\eta}$ paired with a Schwartz function $\varphi(p)$ via

$$
\left(g_{\eta}, \varphi\right)=\left(g, \Omega_{0, \eta} \varphi\right) .
$$

We know that $\Omega_{0, \eta} \varphi$ is a Schwartz function for $\eta \succ 0$, so this definition makes sense. Furthermore it is not hard to check that $\Omega_{0, \eta} \varphi \rightarrow \omega \varphi$ in the Schwartz space topology as $\eta \rightarrow 0, \eta \succ 0$. This proves Parts (a),(b).

Next, let us define

$$
F(\xi, \eta)=\left(g, \Omega_{\xi, \eta}\right), \quad \xi, \eta \in \mathbb{R}^{1, d-1} .
$$

We know that $\Omega_{\xi, \eta}$ is a Schwartz function for $\eta \succ 0$, so $F(\xi, \eta)$ is a function. Moreover it is not hard to show that the family $\Omega_{\xi, \eta}$ is continuous and continuously differentiable in the Schwartz space topology. It also obviously satisfies the Cauchy-Riemann equations: $\left(\partial_{\xi}+i \partial_{\eta}\right) \Omega_{\xi, \eta}=0$. This implies that $F(\xi, \eta)$ is a holomorphic function in $\xi+i \eta$. It remains to show that $F(\xi, \eta)=\widehat{g_{\eta}}(\xi)$. It's enough to check this integrated against a compactly supported test function $\chi(\xi)$ :

$$
\begin{aligned}
\int F(\xi, \eta) \chi(\xi) d \xi & =\int\left(g, \Omega_{\xi, \eta}\right) \chi(\xi) d \xi \\
& =\left(g, \int d \xi \chi(\xi) \Omega_{\xi, \eta}\right)=\left(g, \Omega_{0, \eta} \widehat{\chi}\right)=\left(g_{\eta}, \widehat{\chi}\right)=\left(\widehat{g_{\eta}}, \chi\right) .
\end{aligned}
$$

The proof is complete.

\section{Intuition about lemma 4.2}

The proof of lemma 4.2 in section 4.1 was by contradiction. To help intuition, we will give here a constructive argument of a special case of lemma 4.2 , namely $d=1$ and $n=2$. 
I.e. we will show how any Schwartz function $f \in \mathcal{S}(\mathbb{R})$ can be approximated by Schwartz functions $g$ which for $E \geqslant 0$ agree with Laplace transform:

$$
\mathcal{L}(\varphi)(E)=\int_{0}^{\infty} d t \varphi(t) e^{-E t}
$$

$\varphi \in C_{0}^{\infty}\left(\mathbb{R}_{+}\right)$(compactly supported with support strictly inside $(0,+\infty)$ ), while for $E<0$, $g(E)$ is extended arbitrarily. Recall that the Schwartz space topology is given by the family of norms

$$
|f|_{n}=\sup _{E \in \mathbb{R}, m \leqslant n}\left(1+E^{2}\right)^{n / 2}\left|f^{(m)}(E)\right|,
$$

and we need to find a sequence $\left\{g_{r}\right\}_{r=1}^{\infty}$ such that $\left|f-g_{r}\right|_{n} \rightarrow 0$ as $r \rightarrow \infty$ for any $n$ (we stress that one sequence $g_{r}$ should work for any $n$ ).

We will also consider the Schwartz space $\mathcal{S}\left(\overline{\mathbb{R}_{+}}\right)$, consisting of $C^{\infty}$ functions on $E \geqslant 0$ (not necessarily vanishing at $E=0$ ) with topology given by the family of norms $|f|_{n,+}$ defined by the same equations as (D.2) but with sup taken over $E \geqslant 0$. It will be sufficient to arrange that for any $n$

$$
\left|f-\mathcal{L}\left(\varphi_{r}\right)\right|_{n,+} \rightarrow 0 \quad(r \rightarrow \infty) .
$$

This is because there exists an extension operator which takes a function $h \in \mathcal{S}\left(\overline{\mathbb{R}_{+}}\right)$and provides a function $\mathcal{E}(h) \in \mathcal{S}(\mathbb{R})$ such that $\mathcal{E}(h)=h$ for $E \geqslant 0$ (which is why it called an extension operator), and in addition

$$
|\mathcal{E}(h)|_{n} \leqslant C_{n}|h|_{n,+}
$$

for all $n$ with some finite constants $C_{n}$ independent of $h$. E.g., Seeley's linear extension operator $[135,136]$ has this property. Then, given (D.3), we put

$$
g_{r}=f+\mathcal{E}\left(\mathcal{L}\left(\varphi_{r}\right)-f\right),
$$

which, on the one hand satisfies $g_{r}(E)=\mathcal{L}\left(\varphi_{r}\right)(E)$ for $E \geqslant 0$ and on the other hand by (D.3) and (D.4) has $\left|g_{r}-f\right|_{n} \leqslant C_{n}\left|\mathcal{L}\left(\varphi_{r}\right)-f\right|_{n,+} \rightarrow 0$ which is what we need.

So let us focus on satisfying (D.3). By a map $x=\frac{1}{1+E}$ the half-line $[0,+\infty)$ is mapped to the interval $(0,1]$ and the function $f(E)$ is mapped to a function $F(x)=f\left(\frac{1}{x}-1\right)$ which is a $C^{\infty}$ function vanishing at $x=0$ faster than any power of $x$. For any $\varepsilon$ and any $N$ we can find, by the Weierstrass theorem, a polynomial $Q(x)$ such that

$$
\left|F^{(N)}(x)-Q(x)\right| \leqslant \varepsilon \quad(0 \leqslant x \leqslant 1) .
$$

Let $P(x)$ be the polynomial such that $P^{(N)}(x)=Q(x)$ and $P(0)=\cdots=P^{(N-1)}(0)=0$. Then $P(x)=O\left(x^{N}\right)$ and it is not hard to see that

$$
\left|F^{(n)}(x)-P^{(n)}(x)\right| \leqslant \varepsilon x^{N-n} \quad(0 \leqslant x \leqslant 1) .
$$

We also put $p(E)=P\left(\frac{1}{1+E}\right)$. From $f(E)=F\left(\frac{1}{1+E}\right)$ we know that

$$
\left|f^{(n)}(E)\right| \leqslant B_{n} \max _{m \leqslant n}\left|F^{(m)}\left(\frac{1}{1+E}\right)\right| .
$$


So combining this with eq. (D.7), and going up to $n=N / 2$ we may conclude that

$$
|f-p|_{N / 2,+} \leqslant B_{N}^{\prime} \varepsilon \text {. }
$$

Now, by construction $p$ has the form

$$
p(E)=\sum_{N \leqslant n \leqslant M} a_{n} \frac{1}{(1+E)^{n}} .
$$

Since $\frac{1}{(1+E)^{n}}=\frac{1}{(n-1) !} \int_{0}^{\infty} t^{n-1} e^{-(1+E) t} d t$, we see that $p(E)$ is the Laplace transform of a function $\psi(t)$ :

$$
p=\mathcal{L}(\psi), \quad \psi(t)=\sum_{N \leqslant n \leqslant M} \frac{a_{n}}{(n-1) !} t^{n-1} e^{-t} .
$$

Now we can finish the argument as follows. For $r=1,2,3, \ldots$ we apply the above argument with $N=2 r$ and $\varepsilon=1 /\left(B_{N}^{\prime} r\right)$ to find $\psi_{r}$ such that, by (D.9),

$$
\left|f-\mathcal{L}\left(\psi_{r}\right)\right|_{r,+} \leqslant 1 / r .
$$

The function $\psi_{r}$ is not in $C_{0}^{\infty}(0, \infty)$ although $\psi_{r}^{(k)}=0$ for $k=0 \ldots 2 r-2$, and it vanishes at $\infty$ exponentially. We can therefore approximate $\psi_{r}$ by a $C_{0}^{\infty}(0, \infty)$ function $\varphi_{r}$ so that $\left|\psi_{r}-\varphi_{r}\right|_{2 r-2,+}$ is arbitrarily small, where the order $2 r-2$ of the norm is related to the order of the vanishing of $\psi_{r}$ at $t=0$. Furthermore we have the following lemma:

Lemma D.1. Let $\chi$ be a $C^{\infty}$ function on $[0,+\infty)$ which exponentially vanishes at infinity and

$$
\chi^{(k)}(0)=0, \quad k=0 \ldots n-1 .
$$

Then, with some constant $D_{n}$ independent of $\chi$,

$$
|\mathcal{L}(\chi)|_{n,+} \leqslant D_{n}|\chi|_{n+2,+} .
$$

Proof. We use the following elementary properties of Laplace transform:

$$
\begin{aligned}
\left(\frac{d}{d E}\right)^{m} \mathcal{L}(\chi)(E) & =\mathcal{L}\left[\chi(t)(-t)^{m}\right](E), \\
E^{n} \mathcal{L}(\chi)(E) & =\mathcal{L}\left[\chi^{(n)}(t)\right](E),
\end{aligned}
$$

where the second equation is derived by integration by parts and is valid under (D.13) and exponential decay. So we have (where $\lesssim$ denotes $\leqslant$ with some $n$-dependent but functionindependent constant)

$$
\begin{aligned}
|\mathcal{L}(\chi)|_{n,+} & \lesssim \sum_{m=0}^{n} \sup _{E \geqslant 0}\left(1+E^{n}\right)\left|\mathcal{L}(\chi)^{(m)}(E)\right| \\
& \leqslant \sum_{m=0}^{n} \sup _{E \geqslant 0}\left|\mathcal{L}\left[\chi(t) t^{m}\right](E)\right|+\left|\mathcal{L}\left[\left(\chi(t) t^{m}\right)^{(n)}\right](E)\right|,
\end{aligned}
$$

Using further the elementary bound $|\mathcal{L}(f)(E)| \lesssim \sup _{t \geqslant 0}\left|\left(1+t^{2}\right) f(t)\right|$ we deduce (D.14). 
We use this lemma with $n=r$ and $\chi=\psi_{r}-\varphi_{r}$, which satisfies $\chi^{(k)}=0$ up to $k=2 r-2 \geqslant r-1$, so (D.13) is satisfied. By (D.14), we have

$$
\left|\mathcal{L}\left(\psi_{r}\right)-\mathcal{L}\left(\varphi_{r}\right)\right|_{r,+} \leqslant D_{r}\left|\psi_{r}-\varphi_{r}\right|_{r+2,+} \leqslant D_{r}\left|\psi_{r}-\varphi_{r}\right|_{2 r-2,+}
$$

as long as $r \geqslant 4$ so that $2 r-2 \geqslant r+2$. As mentioned above $\left|\psi_{r}-\varphi_{r}\right|_{2 r-2,+}$ can be made arbitrarily small. Combining with (D.12), we can arrange so that $\left|f-\mathcal{L}\left(\varphi_{r}\right)\right|_{r,+} \leqslant 2 / r \rightarrow 0$ as $r \rightarrow \infty$, which in particular implies (D.3).

Open Access. This article is distributed under the terms of the Creative Commons Attribution License (CC-BY 4.0), which permits any use, distribution and reproduction in any medium, provided the original author(s) and source are credited.

\section{References}

[1] A. Polyakov,History of Recent Science and Technology Project, The Physics of Scale, interview (2003).

[2] K. Osterwalder and R. Schrader, Axioms for Euclidean Green's functions, Comm. Math. Phys. 31 (1973) 83.

[3] K. Osterwalder and R. Schrader, Axioms for Euclidean Green's functions. 2., Commun. Math. Phys. 42 (1975) 281 [INSPIRE].

[4] T. Hartman, S. Jain and S. Kundu, Causality constraints in conformal field theory, JHEP 05 (2016) 099 [arXiv: 1509.00014] [INSPIRE].

[5] R.F. Streater and A.S. Wightman, PCT, spin and statistics, and all that, Benjamin, New York, U.S.A. (1964).

[6] R. Haag and D. Kastler, An algebraic approach to quantum field theory, J. Math. Phys. 5 (1964) 848 [INSPIRE].

[7] R. Haag, Local quantum physics: fields, particles, algebras, Springer, Germany (1992).

[8] A.B. Zamolodchikov and A.B. Zamolodchikov, Factorized S-matrices in two-dimensions as the exact solutions of certain relativistic quantum field models, Annals Phys. 120 (1979) 253 [INSPIRE].

[9] A.A. Belavin, A.M. Polyakov and A.B. Zamolodchikov, Infinite conformal symmetry in two-dimensional quantum field theory, Nucl. Phys. B 241 (1984) 333 [InSPIRE].

[10] R. Rattazzi, V.S. Rychkov, E. Tonni and A. Vichi, Bounding scalar operator dimensions in 4D CFT, JHEP 12 (2008) 031 [arXiv:0807.0004] [INSPIRE].

[11] S. El-Showk, M.F. Paulos, D. Poland, S. Rychkov, D. Simmons-Duffin and A. Vichi, Solving the $3 D$ Ising model with the conformal bootstrap, Phys. Rev. D 86 (2012) 025022 [arXiv:1203.6064] [INSPIRE].

[12] S. El-Showk, M.F. Paulos, D. Poland, S. Rychkov, D. Simmons-Duffin and A. Vichi, Solving the $3 d$ Ising model with the conformal bootstrap II. c-minimization and precise critical exponents, J. Stat. Phys. 157 (2014) 869 [arXiv:1403.4545] [InSPIRE].

[13] F. Kos, D. Poland and D. Simmons-Duffin, Bootstrapping mixed correlators in the $3 D$ Ising model, JHEP 11 (2014) 109 [arXiv:1406.4858] [INSPIRE].

[14] D. Simmons-Duffin, A semidefinite program solver for the conformal bootstrap, JHEP 06 (2015) 174 [arXiv: 1502.02033] [InSPIRE]. 
[15] F. Kos, D. Poland, D. Simmons-Duffin and A. Vichi, Precision Islands in the Ising and $O(N)$ models, JHEP 08 (2016) 036 [arXiv:1603.04436] [INSPIRE].

$[16]$ F. Kos, D. Poland and D. Simmons-Duffin, Bootstrapping the $O(N)$ vector models, JHEP 06 (2014) 091 [arXiv: 1307.6856] [InSPIRE].

[17] F. Kos, D. Poland, D. Simmons-Duffin and A. Vichi, Bootstrapping the $O(N)$ archipelago, JHEP 11 (2015) 106 [arXiv:1504.07997] [INSPIRE].

[18] S.M. Chester et al., Carving out OPE space and precise $O(2)$ model critical exponents, JHEP 06 (2020) 142 [arXiv:1912.03324] [INSPIRE].

[19] S.M. Chester et al., Bootstrapping Heisenberg magnets and their cubic instability, arXiv: 2011.14647 [INSPIRE].

[20] D. Poland, S. Rychkov and A. Vichi, The conformal bootstrap: theory, numerical techniques, and applications, Rev. Mod. Phys. 91 (2019) 015002 [arXiv:1805.04405] [InSPIRE].

[21] M.F. Paulos, J. Penedones, J. Toledo, B.C. van Rees and P. Vieira, The S-matrix bootstrap. Part I. QFT in AdS, JHEP 11 (2017) 133 [arXiv:1607.06109] [INSPIRE].

[22] M.F. Paulos, J. Penedones, J. Toledo, B.C. van Rees and P. Vieira, The S-matrix bootstrap. Part II. Two dimensional amplitudes, JHEP 11 (2017) 143 [arXiv:1607.06110] [InSPIRE].

[23] M.F. Paulos, J. Penedones, J. Toledo, B.C. van Rees and P. Vieira, The S-matrix bootstrap. Part III. Higher dimensional amplitudes, JHEP 12 (2019) 040 [arXiv:1708.06765] [INSPIRE].

[24] L. Córdova and P. Vieira, Adding flavour to the S-matrix bootstrap, JHEP 12 (2018) 063 [arXiv: 1805.11143] [INSPIRE].

[25] A.L. Guerrieri, J. Penedones and P. Vieira, Bootstrapping QCD using pion scattering amplitudes, Phys. Rev. Lett. 122 (2019) 241604 [arXiv:1810.12849] [INSPIRE].

[26] J. Elias Miró, A.L. Guerrieri, A. Hebbar, J. Penedones and P. Vieira, Flux tube S-matrix bootstrap, Phys. Rev. Lett. 123 (2019) 221602 [arXiv: 1906.08098] [INSPIRE].

[27] L. Córdova, Y. He, M. Kruczenski and P. Vieira, The $O(N)$ S-matrix monolith, JHEP 04 (2020) 142 [arXiv: 1909.06495] [InSPIRE].

[28] D. Karateev, S. Kuhn and J. Penedones, Bootstrapping massive quantum field theories, JHEP 07 (2020) 035 [arXiv: 1912.08940] [INSPIRE].

[29] M. Correia, A. Sever and A. Zhiboedov, An analytical toolkit for the S-matrix bootstrap, arXiv: 2006.08221 [INSPIRE].

[30] A.L. Guerrieri, J. Penedones and P. Vieira, S-matrix bootstrap for effective field theories: massless pions, JHEP 06 (2021) 088 [arXiv: 2011.02802] [INSPIRE].

[31] A. Hebbar, D. Karateev and J. Penedones, Spinning S-matrix Bootstrap in 4d, arXiv:2011.11708 [INSPIRE].

[32] P. Tourkine and A. Zhiboedov, Scattering from production in 2d, arXiv:2101.05211 [INSPIRE].

[33] A. Sinha and A. Zahed, Crossing symmetric dispersion relations in quantum field theories, Phys. Rev. Lett. 126 (2021) 181601 [arXiv: 2012.04877] [INSPIRE].

[34] P. Haldar, A. Sinha and A. Zahed, Quantum field theory and the Bieberbach conjecture, SciPost Phys. 11 (2021) 002 [arXiv:2103.12108] [INSPIRE].

[35] Y. He and M. Kruczenski, S-matrix bootstrap in $3+1$ dimensions: regularization and dual convex problem, arXiv:2103.11484 [INSPIRE]. 
[36] P. Kravchuk, J. Qiao and S. Rychkov, Distributions in CFT. Part I. Cross-ratio space, JHEP 05 (2020) 137 [arXiv: 2001. 08778] [INSPIRE].

[37] J. Qiao, Classification of convergent OPE channels for Lorentzian CFT four-point functions, arXiv:2005.09105 [INSPIRE].

[38] S. Rychkov, 3D Ising model: a view from the conformal bootstrap island, Comptes Rendus Physique 21 (2020) 185 [arXiv: 2007.14315] [INSPIRE].

[39] A. Schwarz, Axiomatic conformal theory in dimensions $>2$ and AdS/CT correspondence, Lett. Math. Phys. 106 (2016) 1181 [arXiv:1509.08064] [INSPIRE].

[40] G. Segal, The definition of cft, in Topology, geometry and quantum field theory, U. Tillman ed., Cambridge University Press, Cambridge U.K. (2004),

[41] G. Mack, Convergence of operator product expansions on the vacuum in conformal invariant quantum field theory, Commun. Math. Phys. 53 (1977) 155 [InSPIRE].

[42] P. Kravchuk, J. Qiao and S. Rychkov, Distributions in CFT. Part III. Spinning fields in Minkowski space, to appear.

[43] B. Simon, The $P(\phi)_{2}$ Euclidean (quantum) theory, Princeton University Press, Princeton, U.S.A. (1974).

[44] H. Casini, Wedge reflection positivity, J. Phys. A 44 (2011) 435202 [arXiv:1009.3832] [INSPIRE].

[45] T. Hartman, S. Kundu and A. Tajdini, Averaged null energy condition from causality, JHEP 07 (2017) 066 [arXiv: 1610.05308] [INSPIRE].

[46] V. Glaser, On the equivalence of the Euclidean and Wightman formulation of field theory, Commun. Math. Phys. 37 (1974) 257 [INSPIRE].

[47] S. Minwalla, Restrictions imposed by superconformal invariance on quantum field theories, Adv. Theor. Math. Phys. 2 (1998) 783 [hep-th/9712074] [INSPIRE].

[48] S. Rychkov, EPFL lectures on conformal field theory in $D \geq 3$ dimensions, SpringerBriefs in Physics Springer, Germany (2016) [arXiv:1601.05000] [inSPIRE].

[49] M. Yamazaki, Comments on determinant formulas for general CFTs, JHEP 10 (2016) 035 [arXiv: 1601.04072] [INSPIRE].

[50] J. Penedones, E. Trevisani and M. Yamazaki, Recursion relations for conformal blocks, JHEP 09 (2016) 070 [arXiv:1509.00428] [INSPIRE].

[51] J.C. Jantzen, Kontravariante Formen auf induzierten Darstellungen halbeinfacher Lie-Algebren, Math. Ann. 226 (1977) 53.

[52] A. Dymarsky, J. Penedones, E. Trevisani and A. Vichi, Charting the space of $3 D$ CFTs with a continuous global symmetry, JHEP 05 (2019) 098 [arXiv: 1705. 04278] [INSPIRE].

[53] A. Dymarsky, F. Kos, P. Kravchuk, D. Poland and D. Simmons-Duffin, The 3d stress-tensor bootstrap, JHEP 02 (2018) 164 [arXiv:1708.05718] [INSPIRE].

[54] T. Faulkner, R.G. Leigh, O. Parrikar and H. Wang, Modular Hamiltonians for deformed half-spaces and the averaged null energy condition, JHEP 09 (2016) 038 [arXiv: 1605.08072] [INSPIRE].

[55] P. Kravchuk and D. Simmons-Duffin, Light-ray operators in conformal field theory, JHEP 11 (2018) 102 [arXiv: 1805.00098] [INSPIRE].

[56] S. Caron-Huot, Analyticity in spin in conformal theories, JHEP 09 (2017) 078 [arXiv: 1703.00278] [INSPIRE]. 
[57] J. Maldacena, D. Simmons-Duffin and A. Zhiboedov, Looking for a bulk point, JHEP 01 (2017) 013 [arXiv: 1509.03612] [INSPIRE].

[58] H. Brezis, Functional analysis, Sobolev spaces and partial differential equations, Springer, Germany (2010).

[59] M. Gillioz, Momentum-space conformal blocks on the light cone, JHEP 10 (2018) 125 [arXiv: 1807. 07003] [INSPIRE].

[60] M. Gillioz, Conformal 3-point functions and the Lorentzian OPE in momentum space, Commun. Math. Phys. 379 (2020) 227 [arXiv: 1909.00878] [inSPIRE].

[61] I. Gelfand and G. Shilov, Generalized Functions. Volume 1: properties and operations, Academic Press, New York U.S.A. (1964).

[62] A.M. Jaffe, High-energy behavior in quantum field theory. I. Strictly localizable fields, Phys. Rev. 158 (1967) 1454 [INSPIRE].

[63] F.A. Dolan and H. Osborn, Conformal four point functions and the operator product expansion, Nucl. Phys. B 599 (2001) 459 [hep-th/0011040] [INSPIRE].

[64] F.A. Dolan and H. Osborn, Conformal partial waves and the operator product expansion, Nucl. Phys. B 678 (2004) 491 [hep-th/0309180] [INSPIRE].

[65] D. Pappadopulo, S. Rychkov, J. Espin and R. Rattazzi, OPE convergence in conformal field theory, Phys. Rev. D 86 (2012) 105043 [arXiv: 1208.6449] [InSPIRE].

[66] M. Hogervorst and S. Rychkov, Radial coordinates for conformal blocks, Phys. Rev. D 87 (2013) 106004 [arXiv:1303.1111] [INSPIRE].

[67] A.L. Fitzpatrick, J. Kaplan, D. Poland and D. Simmons-Duffin, The analytic bootstrap and AdS superhorizon locality, JHEP 12 (2013) 004 [arXiv:1212.3616] [INSPIRE].

[68] Wikipedia, Osgood's lemma.

[69] S. Rychkov, Lorentzian methods in conformal field theory.

[70] P. Kravchuk, A Schwarz-Pick lemma for analytic functions on the forward tube, unpublished.

[71] M. Lüscher and G. Mack, Global Conformal Invariance in Quantum Field Theory, Commun. Math. Phys. 41 (1975) 203 [InSPIRE].

[72] P. Kravchuk, J. Qiao and S. Rychkov, Distributions in CFT. Part IV. Lorentzian cylinder.

[73] M.S. Costa, J. Penedones, D. Poland and S. Rychkov, Spinning conformal correlators, JHEP 11 (2011) 071 [arXiv:1107.3554] [InSPIRE].

[74] H.J. Borchers, Field operators as $C^{\infty}$ functions in spacelike directions, Nuovo Cim. 33 (1964) 1600.

[75] R. Jost, The general theory of quantized fields, American Mathematical Society, U.S.A. (1979).

[76] Y. Tomozawa, Local commutativity and the analytic continuation of the Wightman function, J. Math. Phys. 4 (1963) 1240.

[77] N. Bogolubov, A. Logunov, A. Oksak and I. Todorov, General principles of quantum field theory, Springer (2012).

[78] M. Hogervorst, H. Osborn and S. Rychkov, Diagonal Limit for Conformal Blocks in d Dimensions, JHEP 08 (2013) 014 [arXiv:1305.1321] [INSPIRE].

[79] P. Kravchuk, ???, unpublished. 
[80] M. Gillioz, Conformal partial waves in momentum space, SciPost Phys. 10 (2021) 081 [arXiv: 2012.09825] [INSPIRE].

[81] G. Mack, All unitary ray representations of the conformal group $\mathrm{SU}(2,2)$ with positive energy, Commun. Math. Phys. 55 (1977) 1 [InSPIRE].

[82] G.W. Mackey, Unitary group representations in physics, probability and number theory, Benjamin-Cummings, U.S.A. (1979).

[83] V.S. Vladimirov, Methods of the theory of generalized functions, CRC Press, U.S.A. (2002).

[84] F. Riesz and B. Sz.-Nagy, Functional analysis, Frederick Ungar Publishing Co., New York, U.S.A. (1955).

[85] Y.M. Zinovev, Equivalence of the Euclidean and Wightman field theories, Commun. Math. Phys. 174 (1995) 1 [hep-th/9408009] [INSPIRE].

[86] Wikipedia, Post's inversion formula.

[87] J. Glimm, A. Jaffe and T. Spencer, The Wightman axioms and particle structure in the $P(\phi)_{2}$ quantum field model, Ann. Math. 100 (1974) 585.

[88] J. Glimm and A.M. Jaffe, Quantum physics. A functional integral point of view, $2^{\text {nd }}$ edition, Springer, Germany (1987).

[89] A. Abdesselam, A Second-Quantized Kolmogorov-Chentsov Theorem via the Operator Product Expansion, Commun. Math. Phys. 376 (2020) 555 [arXiv: 1604.05259] [InSPIRE].

[90] H. Epstein, Some analytic properties of scattering amplitudes in quantum field theory, in the proceedings of the $8^{\text {th }}$ Brandeis University Summer Institute in Theoretical Physics: particle symmetries and axiomatic field theory, Waltham, U.S.A. (1965).

[91] P. Kravchuk, Analytic completion of 4pt functions in CFT, unpublished.

[92] S. Caron-Huot and J. Sandor, Conformal Regge theory at finite boost, JHEP 05 (2021) 059 [arXiv: 2008.11759] [INSPIRE].

[93] J.P. Eckmann and H. Epstein, Time ordered products and Schwinger functions, Commun. Math. Phys. 64 (1979) 95 [inSPIRE].

[94] S. Rychkov, CFT Osterwalder-Schrader theorem, talk given at the Simons Collaboration on the Nonperturbative Bootstrap Annual Meeting 2019, November 7-8, New York, U.S.A. (2019).

[95] S. Rychkov, CFT Osterwalder-Schrader theorem, https://www.youtube.com/channel/UC4b4DnOK-jrttpYss9zsddw.

[96] D. Simmons-Duffin, Conformal field theory in lorentzian signature.

[97] D.M. Hofman and J. Maldacena, Conformal collider physics: energy and charge correlations, JHEP 05 (2008) 012 [arXiv:0803.1467] [INSPIRE].

[98] C. Cordova, J. Maldacena and G.J. Turiaci, Bounds on OPE coefficients from interference effects in the conformal collider, JHEP 11 (2017) 032 [arXiv:1710.03199] [INSPIRE].

[99] C. Cordova and K. Diab, Universal bounds on operator dimensions from the average null energy condition, JHEP 02 (2018) 131 [arXiv:1712.01089] [INSPIRE].

[100] Z. Komargodski and A. Zhiboedov, Convexity and liberation at large spin, JHEP 11 (2013) 140 [arXiv: 1212.4103] [INSPIRE].

[101] J. Qiao and S. Rychkov, A tauberian theorem for the conformal bootstrap, JHEP 12 (2017) 119 [arXiv: 1709.00008 ] [INSPIRE]. 
[102] D. Simmons-Duffin, The lightcone bootstrap and the spectrum of the $3 d$ Ising CFT, JHEP 03 (2017) 086 [arXiv: 1612.08471] [INSPIRE].

[103] J. Liu, D. Meltzer, D. Poland and D. Simmons-Duffin, The Lorentzian inversion formula and the spectrum of the $3 d$ O(2) CFT, JHEP 09 (2020) 115 [Erratum ibid. 01 (2021) 206] [arXiv: 2007. 07914] [INSPIRE].

[104] S. Caron-Huot, Y. Gobeil and Z. Zahraee, The leading trajectory in the $2+1 D$ Ising CFT, arXiv: 2007.11647 [INSPIRE].

[105] D.M. Hofman, D. Li, D. Meltzer, D. Poland and F. Rejon-Barrera, A proof of the conformal collider bounds, JHEP 06 (2016) 111 [arXiv:1603.03771] [INSPIRE].

[106] T. Hartman, S. Jain and S. Kundu, A new spin on causality constraints, JHEP 10 (2016) 141 [arXiv: 1601.07904 ] [INSPIRE].

[107] J. Polchinski, L. Susskind and N. Toumbas, Negative energy, superluminosity and holography, Phys. Rev. D 60 (1999) 084006 [hep-th/9903228] [INSPIRE].

[108] M. Gary, S.B. Giddings and J. Penedones, Local bulk S-matrix elements and CFT singularities, Phys. Rev. D 80 (2009) 085005 [arXiv:0903.4437] [INSPIRE].

[109] I. Heemskerk, J. Penedones, J. Polchinski and J. Sully, Holography from conformal field theory, JHEP 10 (2009) 079 [arXiv:0907.0151] [INSPIRE].

[110] J. Penedones, Writing CFT correlation functions as AdS scattering amplitudes, JHEP 03 (2011) 025 [arXiv: 1011.1485] [INSPIRE].

[111] T. Okuda and J. Penedones, String scattering in flat space and a scaling limit of Yang-Mills correlators, Phys. Rev. D 83 (2011) 086001 [arXiv:1002.2641] [InSPIRE].

[112] A.B. Zamolodchikov, Conformal symmetry in two-dimensional space: recursion representation of conformal block, Theor. Math. Phys 73 (1987) 1088.

[113] D. Simmons-Duffin, D. Stanford and E. Witten, A spacetime derivation of the Lorentzian OPE inversion formula, JHEP 07 (2018) 085 [arXiv: 1711.03816] [INSPIRE].

[114] J. Liu, E. Perlmutter, V. Rosenhaus and D. Simmons-Duffin, d-dimensional SYK, AdS loops, and $6 j$ symbols, JHEP 03 (2019) 052 [arXiv:1808.00612] [INSPIRE].

[115] C. Cardona and K. Sen, Anomalous dimensions at finite conformal spin from OPE inversion, JHEP 11 (2018) 052 [arXiv: 1806.10919] [INSPIRE].

[116] S. Albayrak, D. Meltzer and D. Poland, More analytic bootstrap: nonperturbative effects and fermions, JHEP 08 (2019) 040 [arXiv: 1904.00032] [INSPIRE].

[117] C. Cardona, S. Guha, S.K. KaNuMIlli and K. Sen, Resummation at finite conformal spin, JHEP 01 (2019) 077 [arXiv: 1811. 00213] [INSPIRE].

[118] L. Iliesiu, M. Koloğlu and D. Simmons-Duffin, Bootstrapping the $3 d$ Ising model at finite temperature, JHEP 12 (2019) 072 [arXiv:1811.05451] [INSPIRE].

[119] L. Iliesiu, M. Koloğlu, R. Mahajan, E. Perlmutter and D. Simmons-Duffin, The conformal bootstrap at finite temperature, JHEP 10 (2018) 070 [arXiv:1802.10266] [INSPIRE].

[120] S. Albayrak, D. Meltzer and D. Poland, The inversion formula and $6 j$ symbol for $3 d$ fermions, JHEP 09 (2020) 148 [arXiv:2006.07374] [INSPIRE].

[121] M. Kologlu, P. Kravchuk, D. Simmons-Duffin and A. Zhiboedov, The light-ray OPE and conformal colliders, JHEP 01 (2021) 128 [arXiv: 1905.01311] [INSPIRE].

[122] M. Kologlu, P. Kravchuk, D. Simmons-Duffin and A. Zhiboedov, Shocks, superconvergence, and a stringy equivalence principle, JHEP 11 (2020) 096 [arXiv:1904.05905] [INSPIRE]. 
[123] C.-H. Chang, M. Kologlu, P. Kravchuk, D. Simmons-Duffin and A. Zhiboedov, Transverse spin in the light-ray OPE, arXiv:2010.04726 [INSPIRE].

[124] L.J. Dixon, I. Moult and H.X. Zhu, Collinear limit of the energy-energy correlator, Phys. Rev. D 100 (2019) 014009 [arXiv: 1905. 01310] [INSPIRE].

[125] G.P. Korchemsky, Energy correlations in the end-point region, JHEP 01 (2020) 008 [arXiv: 1905.01444] [INSPIRE].

[126] R.C. Brower, J. Polchinski, M.J. Strassler and C.-I. Tan, The Pomeron and gauge/string duality, JHEP 12 (2007) 005 [hep-th/0603115] [INSPIRE].

[127] L. Cornalba, Eikonal methods in AdS/CFT: Regge theory and multi-reggeon exchange, arXiv:0710.5480 [INSPIRE].

[128] L. Cornalba, M.S. Costa and J. Penedones, Eikonal methods in AdS/CFT: BFKL Pomeron at weak coupling, JHEP 06 (2008) 048 [arXiv:0801.3002] [INSPIRE].

[129] M.S. Costa, V. Goncalves and J. Penedones, Conformal Regge theory, JHEP 12 (2012) 091 [arXiv: 1209.4355] [INSPIRE].

[130] M. Gillioz, X. Lu, M.A. Luty and G. Mikaberidze, Convergent momentum-space OPE and bootstrap equations in conformal field theory, JHEP 03 (2020) 102 [arXiv:1912.05550] [INSPIRE].

[131] M. Gillioz, X. Lu and M.A. Luty, Scale anomalies, states, and rates in conformal field theory, JHEP 04 (2017) 171 [arXiv:1612.07800] [INSPIRE].

[132] M. Gillioz, X. Lu and M.A. Luty, Graviton scattering and a sum rule for the $c$ anomaly in 4D CFT, JHEP 09 (2018) 025 [arXiv:1801.05807] [INSPIRE].

[133] M. Gillioz, M. Meineri and J. Penedones, A scattering amplitude in Conformal Field Theory, JHEP 11 (2020) 139 [arXiv:2003.07361] [INSPIRE].

[134] V.S. Vladimirov, Methods of the theory of functions of many complex variables, MIT Press, Cambridge, U.S.A. (1966).

[135] R.T. Seeley, Extension of $C^{\infty}$ functions defined in a half space, Proc. Am. Math. Soc. 15 (1964) 625 .

[136] Wikipedia, Whitney extension theorem. 